Illinois State University

ISU ReD: Research and eData

Theses and Dissertations

8-27-2013

\title{
Understanding The Support Needs Of People With Intellectual And Related Developmental Disabilities Through Cluster Analysis And Factor Analysis Of Statewide Data
}

Yuwadee Viriyangkura

Illinois State University, yuwadeev@yahoo.com

Follow this and additional works at: https://ir.library.illinoisstate.edu/etd

Part of the Special Education Administration Commons, and the Special Education and Teaching Commons

\section{Recommended Citation}

Viriyangkura, Yuwadee, "Understanding The Support Needs Of People With Intellectual And Related Developmental Disabilities Through Cluster Analysis And Factor Analysis Of Statewide Data" (2013).

Theses and Dissertations. 75.

https://ir.library.illinoisstate.edu/etd/75

This Dissertation is brought to you for free and open access by ISU ReD: Research and eData. It has been accepted for inclusion in Theses and Dissertations by an authorized administrator of ISU ReD: Research and eData. For more information, please contact ISUReD@ilstu.edu. 


\title{
UNDERSTANDING THE SUPPORT NEEDS OF PEOPLE WITH INTELLECTUAL AND RELATED DEVELOPMENTAL DISABILITIES THROUGH CLUSTER ANALYSIS AND FACTOR ANALYSIS OF STATEWIDE DATA
}

\author{
Yuwadee Viriyangkura
}

195 Pages

May 2014

Through secondary analyses of statewide data from Colorado, the support needs characteristics of people with intellectual and related developmental disabilities (ID/DD) were investigated. Data used in this study were 57 observed variables (i.e., item scores) in seven subscales (i.e., Home Living, Community Living, Lifelong Learning, Employment, Health \& Safety, Social, and Protection \& Advocacy) of the Supports Intensity Scale (SIS), a standardized support needs assessment for a population of people with ID/DD.

A variety of cluster analysis techniques suggested five clusters of people with ID/DD where Cluster 1 means relatively less intense support needs (i.e., low subscale and composite scores) and Cluster 5 means relatively more intense support needs. Prior latent factor models (i.e., a 7-factor model, a 7-factor hierarchical model, a 5-factor model using a subset of SIS items, a 5-factor model using all of SIS items, and a single-factor model) were also examined to investigate the factor structure of the support needs construct using confirmatory factor analysis. Data supported a 7-factor hierarchical model with the SIS seven subscales as first-order factors and a global support needs as a second-order factor. 
UNDERSTANDING THE SUPPORT NEEDS OF PEOPLE WITH INTELLECTUAL AND

RELATED DEVELOPMENTAL DISABILITIES THROUGH

CLUSTER ANALYSIS AND FACTOR ANALYSIS

OF STATEWIDE DATA

YUWADEE VIRIYANGKURA

A Dissertation Submitted in Partial

Fulfillment of the Requirements for the Degree of

DOCTOR OF EDUCATION

Department of Special Education

ILLINOIS STATE UNIVERSITY 
(C) 2014 Yuwadee Viriyangkura 
UNDERSTANDING THE SUPPORT NEEDS OF PEOPLE WITH INTELLECTUAL AND

RELATED DEVELOPMENTAL DISABILITIES THROUGH

CLUSTER ANALYSIS AND FACTOR ANALYSIS

OF STATEWIDE DATA

YUWADEE VIRIYANGKURA

COMMITTEE MEMBERS:

James R. Thompson, Chair

Julia B. Stoner

Stacey J. Bock

Matthew S. Hesson-McInnis 


\section{ACKNOWLEDGMENTS}

Completing my doctoral studies in the Department of Special Education at Illinois State University represents an important chapter in my life. I experienced culture shock and some unexpected circumstances early on, but I have many good memories throughout the course of my stay. I have learned and grown enormously from all of these experiences over the past four years.

I am indebted to my academic advisor, dissertation chair, and invaluable mentor, Dr. James Thompson, who has invested much time, energy, and care in supporting me both academically and professionally. This dissertation represents my best efforts, which I could not have completed without his insight, wisdom, and patience in guiding me through the course of this study. He has played a most significant part in the completion of this dissertation. I appreciate not only his knowledge, but also the understanding he has had for a student who came from the other side of the world to study with him.

I would like to express my gratitude to my committee for their hard work and excellent suggestions. In addition to serving on my dissertation committee, Dr. Julia Stoner modeled effective teaching practices and supported me in many ways. One thing I will always remember is how she included me socially in her classroom activities. Her consideration eased my transition into the American academic world. I also thank Dr. Stacey Bock for her generosity, willingness to share useful information, and great professional support. Dr. Matthew HessonMcInnis spent a great deal of his valuable time with me concerning statistical analyses and my findings report. I learned so much more than I had expected and really appreciate all that he has taught me. I also thank the state of Colorado for data used in this study as well as 
Dr. Jon Fortune who coordinated with the state authorities and shared useful suggestions about this study.

My life in the States would have been much more difficult without my American family, Peggy and Mark Swerdlik. I cannot thank them enough for the enthusiastic encouragement and support they have given me academically and personally. Their kindness made me feel at home when I was so far away from my home in Thailand. Peggy and Mark, I love you both.

I would not have had these invaluable experiences without two important individuals. Dr. Kullaya Kosuwan convinced me to further my education in the States, supported me enormously during the transition, and has influenced the way I view the field of special education in Thailand. Dr. K, I love you too. Another person is Father Adam Gudalefsky who opened my eyes and heart with his extremely positive attitudes toward people with disabilities. He also challenged me to dream big and to make a difference in the field of special education in Thailand.

I am grateful for the unconditional love and support from my family, especially my parents who have much confidence in me. I thank them for instilling in me the value of hard work, perseverance, and the feeling that "I have enough to give." The word "love" does not seem enough to express my feeling.

This work is dedicated to everyone who needs extraordinary supports and their families. Their courage in overcoming barriers and challenges has inspired me to make a difference to my country. I am also thankful that they allowed me to be part of their lives.

Finally, the faculty, staff, and friends at Illinois State University and in the Department of Special Education who directly and indirectly contributed to this meaningful life chapter also deserve my thanks. Although I believe my life over the past several years is the good result of my karma, I would like to thank everyone who has had a positive impact on me during the time I spent in this community. Thank you!

Y.V. 


\section{CONTENTS}

Page

ACKNOWLEDGMENTS

$\begin{array}{lll}\text { CONTENTS } & \text { iii }\end{array}$

TABLES vi

FIGURES vii

CHAPTER

I. THE PROBLEM AND ITS BACKGROUND 1

Statement of the Problem 1

Naming, Defining, and Classifying ID/DD 2

Naming ID/DD 2

Defining ID/DD $\quad 7$

Classifying ID/DD 18

$\begin{array}{ll}\text { Purpose of Current Study } & 27\end{array}$

$\begin{array}{ll}\text { Understanding Supports and Support Needs } & 28\end{array}$

The Supports Paradigm 28

Supports and Support Needs $\quad 29$

Research on the Factor Structure of the SIS 31

$\begin{array}{ll}\text { Purpose of Current Study } & 33\end{array}$

Research Questions $\quad 33$

Definition of Terms

Assumptions $\quad 34$

Limitations of the Study $\quad 34$

$\begin{array}{ll}\text { Chapter Summary } & 35\end{array}$

II. REVIEW OF RELATED LITERATURE 36

History of ID Conceptualization 36

Earliest Work Prior to $1800 \quad 36$

A Professional Field Emerges: 1800 - 1899 
Institutionalization and Scientific Application: 1900 - 1949

The Parent Movement and Deinstitutionalization: 1950 - 1980

Self-Determination, Quality of Life, Inclusion, and Supports: 1980

Onward

$\begin{array}{ll}\text { Classification in ID/DD } & 47\end{array}$

Historical Approaches to Classifying People with ID 47

Purposes Served by Classification $\quad 55$

Classification for the Purpose of Resource Allocation 56

Supports and Support Needs $\quad 59$

Measuring Support Needs Prior to Standardized Measurements $\quad 60$

Current Support Needs Assessment Instruments 62

$\begin{array}{ll}\text { Supports Intensity Scale (SIS) } & 66\end{array}$

SIS Information $\quad 66$

Criticisms of the SIS $\quad 69$

SIS Research Reported in Peer-Reviewed Journals $\quad 71$

SIS Research and Reports in Grey Literature $\quad 84$

Factor Analysis and Cluster Analysis in Special Education 90

Overview of Factor Analysis 90

Applications of Factor Analysis to ID/DD 92

Overview of Cluster Analysis $\quad 95$

Applications of Cluster Analysis to ID/DD and Special Education 96

$\begin{array}{ll}\text { Chapter Summary } & 97\end{array}$

III. RESEARCH DESIGN $\quad 98$

$\begin{array}{ll}\text { Statement of the Problem } & 98\end{array}$

$\begin{array}{lr}\text { Research Questions } & 98\end{array}$

$\begin{array}{lr}\text { Research Design Procedures } & 100\end{array}$

Collection of Data $\quad 100$

$\begin{array}{ll}\text { Measurement } & 102\end{array}$

$\begin{array}{ll}\text { Statistical Measures } & 104\end{array}$

$\begin{array}{ll}\text { Chapter Summary } & 107\end{array}$

$\begin{array}{ll}\text { IV. ANALYSIS OF DATA } & 108\end{array}$

$\begin{array}{ll}\text { Statement of the Problem } & 108\end{array}$

$\begin{array}{ll}\text { Research Questions } & 108\end{array}$ 
Procedures and Findings

Data Cleaning and Preparation 109

Investigating Descriptive Analysis and Reliability of Data 110

Examining Appropriate Number of Clusters 113

Finding Latent Factor Model of Support Needs 123

$\begin{array}{ll}\text { Chapter Summary } & 136\end{array}$

V. CONCLUSION: SUMMARY AND RECOMMENDATIONS 137

$\begin{array}{ll}\text { Summary of Procedures, Findings, and Discussions } & 139\end{array}$

Findings and Implications Related to Research Question 1

Findings and Implications Related to Research Question 2

Implication of Findings for National Goals 152

Goal 1: Increasing Self-Determination and Personal Control in Decisions Affecting People With ID/DD and Their Families 152

Goal 2: Providing Opportunities to People With ID/DD to Live and Participate in Their Own Communities

Goal 3: Improving Quality of Life for Individuals and Families as They Define it for Themselves

Goal 4: Supporting Families as the Most Important and Permanent Unit of Development, Protection, and Lifelong Assistance to Persons With ID/DD

Goal 5: Investing in Each Individual's Developmental Potential and Capacity to Contribute in Age-Related Roles as Productive, Respected Community Members

Goal 6: Assuring Access to Sufficient, High-Quality Health and Social Supports to Protect Each Person's Health, Safety, Rights, and WellBeing

Limitations $\quad 159$

$\begin{array}{ll}\text { Recommendations for Future Research } & 160\end{array}$

$\begin{array}{ll}\text { Chapter Summary } & 161\end{array}$

REFERENCES $\quad 162$

APPENDIX A: Graphic Representations of the Latent Factor Models 181

APPENDIX B: Sample Summary of Finding from SIS Assessment 187 


\section{TABLES}

Table

Page

1. A Resource Allocation Model Proposed for Colorado CW Program

2. Comparison Among Support Needs Assessment Instruments

3. Summary of SIS Research Reported in Peer-Reviewed Journals

$72-77$

4. CW Participants' Demographic Data

5. Colorado's Funding Levels Suggested by the HSRI

6. Results of the Univariate ANOVAs of SIS Scores in the 2-Cluster Solution

7. Results of the Univariate ANOVAs of SIS Scores in the 3-Cluster Solution

8. Results of the Univariate ANOVAs of SIS Scores in the 4-Cluster Solution

9. Results of the Univariate ANOVAs of SIS Scores in the 5-Cluster Solution

10. Personal Information of CW Participants in the 5-Cluster Solution

11. SIS Subscale Scores and SNI of Colorado's CW Participants in the 5-Cluster Solution

12. Intercorrelations Between SIS Subscales

13. Fit Indices of Support Needs Models

14. Standardized Loadings and Squared Multiple Correlations of SIS Items of a 7-Factor Model, a 7-Factor Hierarchical Model, and a Single-Factor Model

15. Standardized Loadings and Squared Multiple Correlations of SIS Items of a 5-Factor Model (Using a Subset of SIS Items) and a 5-Factor Model (Using All SIS Items) 


\section{FIGURES}

Figure $\quad$ Page

1. SIS Subscale Scores and SNI Scores of the Five Clusters 123

2. Percentages of People with ID/DD $(n=3,998)$ in Five Support-Intensity Clusters 142 


\section{CHAPTER I}

\section{THE PROBLEM AND ITS BACKGROUND}

\section{Statement of the Problem}

One of the most salient differences between individuals with intellectual and related developmental disabilities (ID/DD) and the general population is the nature and extent of supports required to fully participate in daily life activities in school and society (Schalock et al., 2010). People with ID/DD require extra support that others do not need to live in their homes, learn in neighborhood schools, work on community jobs, and participate as full members of a modern society. Understanding people with ID/DD by their support needs is essential to planning for and developing appropriate supports that can bridge the gap between people's current life experiences and the life experiences they would like to have (Thompson et al., 2009). Providing supports that are aligned with a person's support needs will lead to improved personal functioning, superior personal outcomes, and an enhanced quality of life (Buntinx \& Schalock, 2010). Arranging supports that address individual needs has become a major focus in the field of ID/DD over the past two decades (Luckasson et al., 1992; 2002; Schalock et al., 2010; Thompson et al., 2002).

Considerable progress has been made in understanding people by their support needs and in planning supports. There is still much to be learned, however, in regard to understanding people by their support needs as well as the nature of supports that truly lead to higher quality lives and improved personal outcomes. This study extended the knowledge base through systematic analyses of a statewide data set of adults with ID/DD. Cluster analysis procedures were applied to identify subgroups within the ID/DD population that share similar support 
needs characteristics. Additionally, confirmatory factor analysis procedures were applied to investigate the empirical support for alternative latent factor models of support needs of people with ID/DD.

\section{Naming, Defining, and Classifying ID/DD}

\section{Naming ID/DD}

Intellectual disability (ID). Luckasson and Reeve (2001) discussed key distinctions between naming a disability, defining a disability, and classifying within a disability. They pointed out that a name (i.e., terminology) is needed so that people can effectively communicate about a disability or disability group. Over time, a disability term can become stigmatizing if others marginalize people with the disability. This has certainly been the case with $I D$. Terms for ID (and subgroups within ID) used over the last 200 years included idiocy, feebleminded, imbecile, moron, trainable mentally handicapped, educable mentally handicapped, mental deficiency, mental disability, and mental retardation (MR). These pejorative terms were clinical terms when first introduced (Schalock et al., 2010; Scheerenberger, 1983; Smith \& Wehmeyer, 2012).

In discussing the change in terminology in the American Association on Intellectual and Developmental Disabilities, ${ }^{1}$ (AAIDD) most recent manual for terminology, definition, and classification, Schalock et al. (2010) provided several reasons why $I D$ was preferred to the term MR. They contended that ID better reflected the construct of the disability because it provided a more logical basis for understanding supports, was aligned better with professional practices and international usage, and was less offensive. It was this final reason (i.e., ID is less offensive) that undoubtedly carried the most weight. In the years leading up to the publication

\footnotetext{
${ }^{1}$ The American Association on Intellectual and Developmental Disabilities (AAIDD) was formerly known as the American Association on Mental Retardation (AAMR), and it underwent several name changes prior to the AAMR. However, in this dissertation, this organization will be referred to as the AAIDD.
} 
of AAIDD's 2010 manual, it was evident that the term MR, and especially its derivations (e.g., retarded, retard), had become derogatory in terms of public use and it was no longer suitable for clinical purposes. In 2009, an organized advocacy effort to end the use of "retarded" resulted in a nationwide campaign called "Spread the Word to End the Word." Also during 2009, Rosa's Law (PL 111-256) was passed by Congress and signed by President Obama to change references in federal law from MR to ID (Special Olympics, 2013).

In a 2007 article announcing that a new term for MR would be used in the next edition of AAIDD's definition manual, however, Schalock and his colleagues were quite clear that the terminology change did not mean that the population of people included in the disability category was changing. To the contrary, they explicitly stated the following:

The term [ID] covers the same population of individuals who were diagnosed previously with mental retardation in number, kind, level, type, and duration of the disability and the need of people with this disability for individualized services and supports. Furthermore, every individual who is or was eligible for a diagnosis of mental retardation is eligible for a diagnosis of intellectual disability. (p. 116) The distinction between naming a disability and defining a disability is important. When a disability term is changed, it is reasonable to ask if the definitional parameters of who is included under the disability category will also change. Changing the criteria for "membership" (i.e., defining the diagnostic parameters) in a disability population, however, is different than changing terminology. A new definition with new diagnostic parameters results in people moving in or out of the disability population, which affects people's access to publicly funded services (e.g., special education, social security income support) as well as legal protections (e.g., civil rights protections under the Americans with Disabilities Act). Schalock et al. (2010) explicitly communicated that ID was a new name but it did not refer to a new population of people. Although terminology was changed in the AAIDD's 2010 manual, the operational 
definition of ID was the same as the operational definition of MR in the prior AAIDD manual (Luckasson et al., 2002).

Even though ID is considered the preferred term in today's world, history suggests that ID may succumb to the same fate as MR and other terms that came before (e.g., feebleminded, mental deficiency). Thompson and Ezell (2005), however, argued that it was not inevitable. It is reasonable to believe that ID can remain a clinical term and not morph into an insult if public attitudes toward the disability and people with the disability are different than in past years.

Furthermore, even if the term ID acquires the same negative connotations of previous terms 30 years from now, changing the name today could still be considered a worthwhile endeavor if it provided advocates with a "season of time" where a less derogatory term was used, as well as a window of opportunity to promote greater tolerance and appreciation of people with this disability.

Developmental disabilities (DD). Closely associated with the term ID is the term $D D$. The pairing of ID and DD is apparent in the names of leading national and international organizations (e.g., AAIDD, International Association for the Scientific Study of Intellectual and Developmental Disabilities or IASSIDD). The division within the Council for Exceptional Children (CEC) that has traditionally focused on ID currently goes under the name of the Division of Autism and Developmental Disabilities (DADD). They include ID under the DD umbrella.

Thompson and Wehmeyer (2008) reported, "The term developmental disability was essentially created by the 91 st United States Congress in 1970 with passage of the Developmental Disabilities Services and Facilities Construction Amendments (P.L. 91-517)” (p. 14). This law, which became known as the DD Act, was intended to promote the growth of adult services in local communities. The Act was part of a nationwide deinstitutionalization policy. At the time, many families with adult members with ID/DD felt they had no other 
option than to commit their adult child to a residential institution because access to services in many local communities was either quite limited or nonexistent. The original definition of DD was "a disability attributable to mental retardation, cerebral palsy, epilepsy, or another neurological condition of an individual found by the Secretary to be clearly related to mental retardation or to require treatment similar to that for mentally retarded individuals" (Breen \& Richman, 1979, p. 3).

Deinstitutionalization for the ID/DD community was (and continues to be) universally regarded as a huge public policy success (see Braddock, Hemp, \& Rizzolo, 2008; Hamelin, Frijters, Griffiths, Condillac, \& Owen, 2011). As the DD Act was renewed to encourage the growth of additional community services and resources, disparate disability groups (e.g., autism, dyslexia) were added to the categorical mix. In the 1977 reauthorization of the DD Act, the categorical approach was abandoned. It was replaced by a functional description that focused on what people could not do independently (e.g., limitations in capacity for independent living) and included an "age of onset" restriction (i.e., prior to age 22; Thompson \& Wehmeyer, 2008).

It is important to note that the term DD was never intended to be a clinical diagnostic term. Rather, it was created as a legal term that described a population of people who had traditionally been served in state-operated residential institutions for people with ID and related conditions, and now were served by community-based disability service organizations. To this day, $\mathrm{DD}$ is a term that refers to a population of people who receive services from disability service systems (Thompson \& Wehmeyer, 2008). This is an important distinction; the term DD denotes a specific status (i.e., this person is a service recipient) whereas clinical terms (e.g., ID) are aligned with specific characteristics associated with a disability diagnosis.

There is overlap between the ID and DD populations, but these two populations are not the same. Larson, Lakin, Salmi, Scotte, and Webster (2010) presented data indicating that there 
were many people who qualified for a diagnosis of ID but were not served by the adult service system nor included in the population that is described in the DD Act, and there were many others covered under the DD Act who did not meet criteria for an ID diagnosis. Although a discussion of the reasons why this is the case is beyond the scope of this dissertation, it is relevant to point out that people with ID and people receiving services through state DD agencies are intersecting populations but are not the same population. What the two groups share in common is a need for extra supports that most others in modern society do not need (Thompson \& Wehmeyer, 2008).

This study uses data on adults served by the Colorado Division for Developmental Disabilities (CDDD). This study, however, is conceptually grounded on the premises and approaches to understanding ID that have been presented in the last three AAIDD manuals (Luckasson et al., 1992; 2002; Schalock et al., 2010). Therefore, the term ID/DD is used throughout this dissertation to refer to the population of interest. For many years, the term ID/DD has been used in the professional literature by researchers conducting investigations based on group data from governmental jurisdictions (e.g., see Braddock et al., 2008; Larson et al., 2010; Ward, Nichols, \& Freedman, 2010). ID/DD refers to a population with whom practicing professionals work on a daily basis whose primary disability is not related to a physical disability, sensory disability (i.e., vision, hearing), or mental health issues. People with ID/DD are in need of extraordinary supports to participate in life activities and experience life conditions that are valued by the vast majority of others in society (e.g., live in community housing, contribute to society through work in community settings, use community services that are open to the public such as stores, banks, and public transit; see Braddock et al., 2008). 


\section{Defining ID/DD}

Definitional issues. A disability definition is "the cornerstone for the existence of the condition" (p. 93) according to Ysseldyke, Algozzine, and Thurlow (2000). They pointed out there are no identifiable disability categories without definitions. In one sense, a disability does not exist until it is named and defined. For example, prior to the mid-1960s, the disability condition known as learning disability (LD) was not officially recognized by any federal education agency. Special education services for children with LD were few and far between because diagnoses of LD were rare. Once the federal government published a definition of LD and public funds were available to provide special education to children with characteristics aligned with the LD definition, however, diagnoses of LD skyrocketed (Myers \& Hammill, 1990). According to the most recent data released by the U.S. Department of Education (2011), 44.6\% of children (ages 6-21) with Individualized Educational Programs (IEPs) in U.S. public schools have a primary diagnosis of LD.

Similarly, a leading explanation for the dramatic increase in the prevalence of children receiving services under the disability category of autism over the past 20 years has been the introduction of autism as a distinct disability category in the Individuals with Disabilities Education Act in 1992. The conceptualization of autism as a spectrum disorder has been broadened and the number of individuals with this condition dramatically increased (Autism Society, 2012; Newschaffer, Falb, \& Gurney, 2005). As seen from the above examples, a disability population becomes established when a critical mass of people accept a definition as a valid description of a segment of society.

Early definitions of ID. ID has a much longer definitional history than other disability conditions with the exception of sensory (i.e., deaf and hard of hearing, low vision and blindness) and physical disabilities. Terminology and definitional criteria, however, have changed over time (Schalock et al., 2010; Scheerenberger, 1983). Prior to 1900, clinical 
judgment from a medical doctor was used for diagnosing ID. Although the first published definitions of ID emerged during the 1700s, no specific definition was universally accepted and there was no such thing as a definitional manual that was written specifically to guide the work of diagnosticians. A review of early definitions, however, has revealed a general consensus that ID was evidenced by deficits in learning, community adaptation, and social participation (Bruininks, Thurlow, \& Gilman, 1987; Smith \& Wehmeyer, 2012). Although formal statistics were not kept, it is evident that the ID population was proportionally a smaller segment of the population during the 1800 s compared to the 1900 s when IQ testing became common. The population of people with ID was smaller in the 1800 s because definitions of ID were narrower. For instance, a lack of academic achievement was not considered to necessarily be indicative of ID in the 1800s because a large segment of adult society could not read nor write (MacMillan, 1977; Scheerenberger, 1983).

IQ-based definitions of ID. In the early 1900s, the diagnosis of ID dramatically changed when standardized, norm-referenced assessments to measure intelligence (i.e., IQ tests) were introduced. Through information gathered from IQ tests, psychologists took the lead role away from physicians in diagnosing ID. IQ tests purported to measure the psychological construct of intelligence, and ID became defined and understood as deficits in overall mental capacity (i.e., raw mental firepower) in contrast to deficits in the performance of activities of daily life that were prominent in the earlier definitions (Scheerenberger, 1983; Smith \& Wehmeyer, 2012).

Additionally, IQ tests produced concrete scores and were perceived to be a less subjective means to diagnose ID compared to the clinical judgments and opinions of individual physicians. Although language in published definitions continued to reference social participation (e.g., vocational adjustment) and biological factors (e.g., incomplete cerebral development), evidence of a low IQ score clearly became the most important criteria for 
operationally defining and diagnosing ID. When the AAIDD's committee on the Classification of the Feeble-Minded presented recommendations at the Association's 1910 annual meeting, results from intelligence testing were touted as the most valid means for diagnostic purposes. This committee's work eventually led to the publication of what is considered to be the first AAIDD manual for terminology, definition, and classification of ID in 1921 (Grossman, 1983; MacMillan, 1977; Scheerenberger, 1983; Smith \& Wehmeyer, 2012). Although IQ tests were certainly an efficient and concrete way to collect diagnostic evidence, by the early 1920 s numerous concerns were raised about the validity of the IQ test as a diagnostic tool. Of special concern was the narrowness of items on the IQ test. Lippmann (1922) wrote the following:

It is not possible, I think, to imagine a more contemptible proceeding than to confront a child with a set of puzzles, and after an hour's monkeying with them, proclaim to the child, or to his parents, that here is a C-individual. It would not only be a contemptible thing to do. It would be a crazy thing to do, because there is nothing in these tests to warrant a judgment of this kind. (p. 215)

Criticisms of the IQ score as a measure of intelligence continued to gain momentum as time went on, and this criticism continues to the current day (Williams, 2009). The most scathing criticism has been directed toward those who claim that aggregate IQ scores provide evidence supporting the intellectual superiority and inferiority of different racial and ethnic groups (e.g., see Gould, 1981). Although data showing group differences in IQ scores has raised intense passions, however, the use of IQ scores as a measure of the intelligence of individuals has also been very controversial. By the 1950s, there was great concern that IQ scores were responsible for misdiagnosing children from poor and minority backgrounds with ID (Reschly, Myers, \& Hartel, 2002). Although an in-depth discussion of IQ controversies is beyond the scope of this dissertation, it is important to note that (a) the IQ score continues to 
have a prominent role in informing diagnostic decisions for ID (Schalock et al., 2010) and (b) for well over half of a century there has been consensus among the leading researchers in the area of human intelligence that intelligence is multidimensional, and it must be interpreted in a much broader context than any IQ score can provide (e.g., see Flynn, 2007; Sternberg, 2005).

Adaptive behavior (AB) dual criteria definitions. Reflecting a growing uneasiness with using a lone IQ score to define and diagnose ID, Edgar A. Doll (1941) was among the first to suggest multiple criteria for diagnosing ID. He contended that ID was evidenced by "(1) social incompetence, (2) due to mental subnormality, (3) which has been developmentally arrested, (4) which obtains at maturity, (5) is of constitutional origin, and (6) is essentially incurable" (p. 215). It is noteworthy that only the second of Doll's criteria involved consideration of an IQ score. Today, most researchers in the field of ID would agree that some of Doll's criteria (e.g., understanding the importance of social competence) placed him ahead of his time. Other criteria (e.g., incurability), however, have not proven to be as valid. The critical point to take away from Doll's work is that by the 1940s it was apparent that an IQ score was not in and of itself perceived by the leading researchers of the day to be a satisfactory or sufficient means by which to understand people with ID, base a definition, or make a diagnosis.

In contrast to IQ tests, which purported to measure mental ability, Doll (1935) and others introduced the concept of $\mathrm{AB}$ and began developing $\mathrm{AB}$ scales that focused on measuring achievement instead of measuring a human trait (i.e., intelligence). In one sense, the field had returned to a pre-IQ era where the focus was on assessing deficits in learning, community adaptation, and social participation. Instead of physicians making clinical judgments from informal interviews, however, the concepts were delineated and measured in AB scales. AB assessments focused on measuring a person's competence in everyday life skills. Measuring typical performance, not maximal performance, was stressed (e.g., Could someone cross a busy street safely on a regular basis? - not just be successful on his or her best day). The emphasis of 
$\mathrm{AB}$ assessment was squarely on what a person could and could not do at the time of the assessment, and $\mathrm{AB}$ scales made no claims about innate ability or human potential (Nihira, 1999; Thompson, McGrew, \& Bruininks, 1999).

The AAIDD's 1959 and 1961 manuals for terminology, definition, and classification broke new ground by requiring both an assessment of AB skills and IQ to make a diagnosis of ID (Heber, 1959; 1961). The 1959 manual was reprinted with minor corrections in 1961, and for the sake of clarity this manual will be referred to as the AAIDD 1961 manual in this dissertation. There were two important aspects of the 1961 manual: (a) dual criteria for diagnosis - there needed to be evidence of concurrent deficits in intelligence (as measured by an IQ test) and $\mathrm{AB}$ (as measured by an $\mathrm{AB}$ scale) and (b) increasing the upper IQ limit for diagnosis - it went from two standard deviations (SDs) below the mean to just one SD.

The dual criteria change in 1961 must be considered in the context of other events that were happening in the field at the time. By the late 1950s, there was increasing dissatisfaction in the U.S. with the practice of institutionalizing children and adults with ID/DD. Many were calling for free and appropriate special education services in the public schools as well as for laws establishing community-based services for adults as a means to prevent institutionalization (Scheerenberger, 1983). According to Nihira (1999), "Adaptive behavior assessment offered a technical basis for implementing deinstitutionalization and community rehabilitation. The major contribution of the adaptive behavior approach during the 1960s was to provide socially relevant goals and aspirations for treatment and intervention efforts" (p. 9).

Heber (1961) and the committee with whom he worked were confident that an AB assessment would serve as a counterbalance to the IQ score and thereby lessen the likelihood of Type 1 (i.e., incorrectly diagnosing someone with the condition who in truth does not have it) and Type 2 (i.e., incorrectly diagnosing someone as not having the condition who actually has it) diagnostic errors. Much of the AAIDD's justification in changing the definition (and 
diagnostic parameters) of ID in its 1961 manual from an IQ score that was two SDs below the mean (i.e., approximately 70) to an IQ score that was only one SD (i.e., approximately 85) was predicated on the assumption that requiring evidence of concurrent deficits in $\mathrm{AB}$ and intelligence would prevent an influx of individuals with relatively higher IQs into this disability population. Put another way, it was thought that many people with IQs in the 71-85 range (as well as some with lower IQs) would be adapting successfully in school and in society, which would be reflected by their $\mathrm{AB}$ scores. Therefore, such people would not be diagnosed with ID because of the dual criteria. Others with IQs in the 71-85 range who showed concurrent deficits in $\mathrm{AB}$, however, would be diagnosed and subsequently provided with the help they needed (MacMillan, 1977; Scheerenberger, 1983). In reflecting on the AAIDD's 1961 definition, MacMillan (1977) stated, "Clearly this definition was designed to include more individuals functioning at a marginal level, so that, if they were having problems in adapting, they would be eligible for needed services" (p. 37).

In retrospect, expanding the IQ limit upward exacerbated concerns about misdiagnosing children from minority and culturally different backgrounds (MacMillan, 1977; Scheerenberger, 1983). Based on theoretical IQ distribution, the 1961 definition increased the potential prevalence of ID more than fivefold, from less than $3 \%$ to nearly $17 \%$ of the population! Requiring evidence of concurrent $\mathrm{AB}$ turned out not to be an effective counterbalance to such a dramatic rise in the IQ upper limit. Several court cases were filed in jurisdictions that adapted the 1961 criteria where plaintiffs charged that IQ tests were not appropriate to use with culturally different populations (MacMillan, 1977; Murdick, Gartin, \& Crabtree, 2007).

When the AAIDD published its next manual (Grossman, 1973), it was noteworthy that the AAIDD's 1973 narrative definition of ID was identical to the 1961 manual's, except for inserting the word significantly. According to Grossman, "Mental retardation refers to significantly [emphasis added] subaverage general intellectual functioning existing concurrently 
with deficits in adaptive behavior, and manifested during the developmental period" (p. 5). Operationally, the word significantly meant an extra SD because the AAIDD changed the IQ upper limit back to two SDs below the population mean. The AAIDD, however, continued to maintain the dual criteria requiring evidence of concurrent deficits in $\mathrm{AB}$ and intelligence. Although there have been periodic calls for removing AB from definitions of ID and diagnostic criteria (Clausen, 1972; Greenspan, 1997; Zigler, Balla, \& Hodapp, 1984), the dual criteria for diagnosis have been maintained to the current day (Schalock et al., 2010).

Social-ecological definition. According to Schalock et al. (2007), defining a disability term requires explaining the meaning of the term precisely as well as establishing the boundaries. The distinction between explaining meaning and establishing boundaries becomes critical when aligning a narrative definition with the evidence required to make a diagnosis. The AAIDD's 1992 manual (Luckasson et al.) was revolutionary because it changed the meaning of ID from a deficit trait that resides inside of a person to a state of impaired functioning. The social-ecological conceptualization of ID in the 1992 manual focused on the mismatch between personal competence and environmental demands, not on defects inside a person. Core diagnostic criteria and boundaries in the 1992 manual, however, remained consistent with the AAIDD's 1973 and 1983 manuals (Grossman). Therefore, the 1992 AAIDD manual introduced a social-ecological understanding of ID (meaning) but retained deficit-based criteria for diagnosis (boundaries). The disconnect between meaning and diagnostic criteria continued with publication of the AAIDD's 2002 (Luckasson et al.) and 2010 (Schalock et al.) manuals.

In the AAIDD's 1992 manual, the authors stated that ID "is not a trait .... Rather, mental retardation is a state in which functioning is impaired ... This distinction between trait and state is central to understanding how the present definition .... shifts the emphasis from measurement of traits to understanding the individual's actual functioning in daily living" 
(Luckasson et al., 1992, p. 10). The relationship between people's support needs and their functioning was also addressed. "The needs for support reflect how the individual functions and that the presence or absence of supports can reciprocally influence functioning .... supports are not actually part of the concept of mental retardation but, rather, are responses to it" (p. 11).

Luckasson and colleagues were not alone in the early 1990s when they suggested that disability be understood in a social-ecological context (i.e., disability is a person-environment mismatch) in contrast to a traditional, medical context (i.e., disability is a defect within the person). The fundamental premises of a social-ecological understanding of ID had been introduced in prior years (see Mercer, 1979). Additionally, considerable literature had emerged in the field of disability studies regarding social-ecological conceptual models of disability. For example, Pope and Tarlov (1991) wrote that disability was the "expression of a physical or mental limitation in a social context - the gap between a person's capabilities and the demands of the environment" (p. 1). The 1992 AAIDD manual, however, was groundbreaking because it was the first time that a social-ecological conceptualization of ID had been so comprehensively presented.

Many praised the 1992 manual's focus on how people functioned in daily life and the focus on how supports were the key to improving human functioning and life experiences (see Hodapp, 1995; Polloway, 1997; Smith, 1997). Others, however, did not receive it positively. Some critics did not believe the social-ecological conceptualization of ID was useful (e.g., see Baumeister, 2006; Jacobson \& Mulick, 1996), whereas others were more concerned about operational criteria for diagnosis and other aspects of the 1992 manual that were not specifically related to the new, social-ecological focus (e.g., see Greenspan, 1997; MacMillan, Gresham, \& Siperstein; 1993; 1995). Much of the concern was with the changes that were made to guide assessment processes. The 1992 manual replaced the concept of AB with the adaptive skills in an effort to more operationally define the term. Specifically, 10 adaptive skills (i.e., 
Communication, Self-care, Home Living, Social Skills, Community Use, Self-direction, Health and Safety, Functional Academics, Leisure, and Work) were included in the definition (Luckasson et al., 1992). Critics pointed out that although AB was a multidimensional construct, there was no empirical support for 10 distinct skill areas (Thompson, McGrew, \& Bruininks, 1999).

There was also considerable concern with the perception that the AAIDD's 1992 manual called for an upper IQ limit in the range of 70 to 75 . Although the authors may have intended to provide flexibility "to reflect the statistical variance inherent in all intelligence tests and the need for clinical judgment by a qualified psychological examiner" (Luckasson et al., 1992, p. 14), there was a concern that an IQ score of 80 might qualify a person for an ID diagnosis because the standard error of measurement of some IQ tests is as large as five points. MacMillan et al. (1993) pointed out that due to the normative distribution of IQ, "more people fall in the IQ interval 71 to 75 (i.e., 2.80\%) than in the entire range previously associated with mental retardation (IQ 70 and below; i.e., 2.68\%)" (p. 327). Another feature of the 1992 manual that engendered considerable controversy was the elimination of classification by level of severity based on IQ scores (i.e., Mild [55-70], Moderate [40-54], Severe [25-39], and Profound [24 or lower]; Jacobson \& Mulick, 1996; MacMillan et al., 1993; 1995).

The AAIDD's 2002 manual (Luckasson et al.) addressed many of the critiques leveled at the 1992 manual, but it held firm to the social-ecological conceptualization of ID as a state of functioning that was evidenced by a mismatch between people's competence and environmental demands, and by the need for extraordinary supports that others in society do not need. Some changes to the 2002 manual included dropping mention of a 70-75 upper IQ limit. Instead, the 2002 manual specified the upper IQ limit to be two SDs below the mean while emphasizing how important it was for diagnosticians to consider an IQ tests' standard error of measurement when interpreting IQ scores. There was also an acknowledgment that a classification typology 
should be driven by the purpose served by classifying, and that multiple classification schemes could be appropriate depending on different purposes. Classification by intensity of support needs, severity of deficit, and biological characteristics were provided as examples of useful classification approaches based on differing rationale for classifying. Additionally, the 2002 manual dropped the 10 adaptive skill areas in favor of three broad domains of AB for which there was empirical justification (Luckasson et al., 2002).

As was discussed previously, in the latest edition of the AAIDD manual (Schalock et al., 2010) there was considerable concern that changing the terminology from MR to ID not result in a changing the composition of the disability population. Therefore, the narrative text of the 2010 definition was identical to the 2002 manual, except the word ID was substituted for MR. The current definition of ID is:

Intellectual disability is characterized by significant limitations both in intellectual functioning and in adaptive behavior as expressed in conceptual, social, and practical adaptive skills. This disability originates before age 18 . The following five assumptions are essential to the application of this definition:

1. Limitations in present functioning must be considered within the context of community environments typical of the individual's age peers and culture.

2. Valid assessment considers cultural and linguistic diversity as well as differences in communication, sensory, motor, and behavioral factors.

3. Within an individual, limitations often coexist with strengths.

4. An important purpose of describing limitations is to develop a profile of needed supports.

5. With appropriate personalized supports over a sustained period, the life functioning of the person with intellectual disability generally will improve. (p. 1) 
A critical contribution of the 2010 manual was to explicitly acknowledge the incongruence between the social-ecological conceptualization of ID (meaning) and the deficitbased criteria used for diagnosis (boundaries). The authors recognized that their manual provides two definitions: an operational definition and a constitutive definition. The operational definition makes the manual workable for practicing diagnosticians by keeping the three diagnostic criteria from prior definitions: intellectual functioning, $\mathrm{AB}$, and age of onset. It is noteworthy that two of the criteria are based on measuring deficits (intellectual and $\mathrm{AB}$ deficits) while the third is developmentally based (must occur prior to age 18). All three criteria, however, reside within the person. The operational definition is focused on the operations by which ID can be observed and measured. It is useful for purposes of diagnosis and for classification by deficit level (Schalock et al., 2010).

The constitutive definition is aligned with the social-ecological conceptualization of ID and is focused on defining ID in terms of its theoretical underpinnings. The authors use the change in terminology to underscore the change in the meaning of ID. According to Schalock et al. (2010), "The major difference is in regard to where the disability resides: the former construct (mental retardation) viewed the disability as a defect within the person, whereas the current construct (ID) views the disability as a fit between the person's current capacities and the context in which the person is to function" (p. 13). The 2010 AAIDD manual authors indicated that the constitutive definition provided a more useful approach to understanding people with ID because it directed time and energy to efforts that enhance human functioning through the provision of individualized supports.

A discussion of the multidimensional conceptual framework of human functioning on which Schalock et al.'s (2010) constitutive definition is based is beyond the scope of this dissertation. The major premise of the conceptual framework in the most recent AAIDD manual, however, is that when ID is considered to be a poor fit between a person's competency 
and the environmental context in which the person functions, then professional efforts are not directed toward fixing the person but rather toward providing supports that bridge the gap between "what is" and "what can be." According to the 2010 AAIDD manual, individualized supports play an important role in improving human functioning, personal outcomes, and quality of life of people with ID/DD. Therefore, it is critical that people with ID/DD are understood by their individual support needs (even though diagnosis is completed by collecting information related to a person's deficits).

\section{Classifying ID/DD}

Classification issues. Classifying involves "dividing into groups what has been included within the boundaries of a name or term" (Luckasson \& Reeve, 2001, p. 50). People with ID are a heterogeneous population. Simply knowing that someone has been diagnosed with ID provides very little insight into what a person does and does not do, and it provides absolutely no insight regarding other important personal factors such as preferences, motivation, health status, or behavioral patterns. Therefore, it is not surprising that there has been a long history of efforts to subdivide the ID population into various groups that share common characteristics or needs.

Etiological classification. Classification by etiology (i.e., biological cause) was the first approach to classification and was consistent with the primacy of physicians and the medical field. During the 1860s a British physician, Dr. Langdon Down, made a breakthrough in etiological classification by noting physical characteristics that were shared by a significant number of people with ID. These included round faces with almond shaped eyes; small, flat noses; small mouths with protruding tongues; and small ears. He also noticed short, stubby fingers as well as a single crease across the palm of the hands. Because almond shaped eyes are characteristic among Asian populations, and because the single crease is much more common in people of Asian descent than in others, Dr. Down termed the syndrome Mongolism in reference 
to the Mongolian section of Asia. He went on to posit that various forms of ID were related to genetic regressions associated with different races and began to develop a classification approach based on this idea. He, however, eventually abandoned his "ethnic classification" when his data simply did not provide sufficient evidence for it. Today he is best remembered as the first doctor to comprehensively describe Down syndrome (Harris, 2006; Scheerenberger, 1983).

Although the pioneers of etiological classification completed their work by collecting observations of outward physical characteristics, many medical advances over the years have led to increasingly sophisticated methods for identifying biologically based conditions (e.g., genetic and chromosomal analyses through blood testing, identifying brain malformations through neuroimaging). Although hundreds of specific syndromes associated with ID have been identified, there is no known cause in over half of the cases. Moreover, in the vast majority of cases where there is a known cause, the identification of the cause is not particularly relevant to behavioral interventions or planning. For these reasons, classification by etiology has not been particularly useful for purposes other than those associated with medical research and medical treatments (Durkin \& Stein, 1996).

For medical researchers, however, etiological classification is absolutely necessary. The most dramatic example of the value of etiological classification is Phenylketonuria (PKU), a condition occurring in about 1 of every 10,000 births. Children with PKU experience a build up of a body chemical called phenylalanine. When the condition is left unchecked, severe brain damage and ID result. If, however, PKU is identified at birth, then ID can be completely prevented as long as the child follows a special diet. Nothing can be done to repair a child's central nervous system after it has been damaged; therefore, getting an infant on a special diet (which precludes mother's milk) is critical (Dykens, Hodapp, \& Finucane, 2000). The virtual elimination of PKU as a cause of ID in developed countries over the past fifty years provides an 
important reminder of the usefulness of classification of ID by etiology for purposes of medical intervention.

Intellectual deficit classification. The impact of the AAIDD's 1910 Committee on Classification of the Feebleminded in establishing the IQ test as the prominent diagnostic tool in the field of ID was mentioned earlier. This committee's support for IQ testing, however, did not end at identification. The committee saw the IQ test as an objective means to classify people with ID into groups based on the severity of impairment. Because IQ testing was perceived to be a valid and reliable way to identify people with ID, it was reasoned that it would also be a valid and reliable way to classify this population (Durkin \& Stein, 1996; Harris, 2006).

Working with mental age scores, the 1910 committee suggested the following three categories for people with relatively high, mid-range, and low scores respectively: Morons, Imbeciles, and Idiots (Smith \& Wehmeyer, 2012). In 1916, Professor Lewis Terman of Stanford University, who had taken the lead in developing the newly published Stanford-Binet intelligence test, eliminated difficulties that were inherent to using mental age scores by incorporating the Intelligence Quotient or IQ score into the testing results. Based on IQ scores, he suggested the following classification typology: 80-89 (Dullness), 70-79 (Borderline retardation), 50-69 (Moron), 25-49 (Imbecile), and < 25 (Idiot).

As the years went on, IQ cut-off scores and names for different categories varied based on the organization publishing the guidelines. Many state education agencies and schools adopted a 4-level classification system where children with IQ scores above 70 were referred to as Borderline, those with scores in the 55-70 range were called Educable, children with IQs from 30 to 55 were identified as Trainable, and those with IQ scores less than 30 were called Severe, Profound, or Totally Dependent (see Gearheart \& Litton, 1975; Kirk, 1972; MacMillan, 1977). The Borderline category fell out of use by the mid-1970s due to a combination of factors. Three of the most important were (a) considerable concern about misdiagnosing 
children from poor and minority backgrounds who were significantly overrepresented in terms of the Borderline diagnoses; (b) the AAIDD's 1973 manual which changed the IQ upper limit cutoff score from one to two SDs below the mean, and therefore effectively removed the Borderline group from the field's leading ID definitional manual; and (c) the 1975 passage of PL 94-142 (the federal law establishing that students with disabilities had the right to a free and appropriate public education) which included the disability category of LD. LD had been steadily gaining acceptance and use in schools for over a decade, and the criteria for diagnosing it was sufficiently imprecise to enable many children with IQ scores in the Borderline range to be diagnosed with LD (Reschly et al., 2002; Thompson \& Wehmeyer, 2008; Ysseldyke et al., 2000).

Although some of the classification terminology used in the past (e.g., Imbecile) may seem particularly harsh to today's ears, it is important to remember that these terms were clinical terms at the beginning and were not as pejorative as they seem today. The terms used by educators, denoting that some children were educable but that others were only trainable, could be considered to be the worst of the collection of archaic terms because these terms implied expectations. For approximately the past 20 years the following 4-tier classification system, based on IQ scores, has been widely used by professional organizations, state educational agencies, state DD service agencies, and international jurisdictions: Mild (two SDs below the mean), Moderate (three SDs), Severe (four SDs), and Profound (five or more SDs; see APA 2000; Harris, 2006; Schalock et al., 2010).

A primary reason for using IQ scores for categorizing by level of deficit is that IQ scores facilitate communication among professionals. Professionals working in different jurisdictions and in different disciplines are familiar with IQ scores, and so everyone knows what each other is talking about. There are not many other diagnostic measures that are as accessible as the IQ score (i.e., it is understood the same way in Illinois as it is in Toronto as it 
is in Bangkok). Also, IQ scores are relatively stable and therefore IQ scores produce a classification designation that is not likely to change quickly (Durkin \& Stein, 1996). Although it is conceivable that an $\mathrm{AB}$ score or alternative achievement measures could be used to classify people by deficit level, other deficit-based alternatives to the IQ score simply have not emerged in the professional literature or in practice. The Inventory for Client and Agency Planning (ICAP) is an AB scale that produces a service score. Although the service score identifies level of care requirements, it does not place people into deficit categories per se (Bruininks, Hill, Weatherman, \& Woodcock, 1986).

A second concern is that deficit-level based categories emphasize deficits and do not account for relative strengths. Everyone has relative strengths and weaknesses. Some people with ID are physically strong and coordinated, others have strong interpersonal skills, others have specific areas of academic strength, and so forth. Because IQ tests do not account for AB skills or provide a profile of personal strengths, deficit-based categories that are aligned with IQ scores create a false picture of homogeneity among people within a category. Understanding people by relative strengths and weaknesses, personality traits, health characteristics, and the many other personal factors (that are either more important, or just as important, as an IQ score) would seem to be a far more useful approach than simply focusing on a shared level of deficit (Durkin \& Stein, 1996; Schalock et al., 2010). Schalock et al. pointed out, "Within an individual, limitations often coexist with strengths" (p. 1); a reality that is not reflected in a deficit-based classification tier that is tied to an IQ range.

Perhaps the most significant problem with categorizing people with ID by deficit level has been the tendency of educators, adult service provider staff, and other professionals to align service options with deficit classification categories. Up until the 1970s, it was common for there to be classrooms, and even entire schools, designed for specific categories of students (e.g., the "Educable Mentally Handicapped classroom" and the "Trainable Mentally 
Handicapped school"). Additionally, many community-based service providers established sheltered workshop programs as well as residential programs for people in specific deficit-level categories (see Gearheart \& Litton, 1975; MacMillan, 1977). The principle of normalization, the mainstreaming and inclusive education movements, and the supported employment and supported living initiatives of the past 60 years have all been important influences in challenging the viability of "special programs in special settings" based on disability classifications. Although the importance of basing settings, activities, or services on each person's unique needs and interests (and therefore not on disability labels and classifications) has been a consistent theme of professional literature for at least 30 years, there is ample evidence to suggest that many special education and adult service programs continue to be conceptually aligned with a deficit-based classification approach (see The Arc, 2011; U.S. Department of Education, 2011).

As mentioned previously, the AAIDD 1992 manual eliminated deficit-based classification, but it was restored as an option in the 2002 and 2010 manuals. The reason for its reinstatement was that classification approaches should be driven by purpose. The 2002 manual's authors acknowledged that some useful purposes could be served by deficit-based classification. For instance, researchers interested in an intervention's effectiveness often do not have access to specific IQ scores, but they are able to access IQ score ranges. In such a case, because ID is such a heterogeneous population, it would be important for the researchers to identify the characteristics of the people for whom an intervention was or was not successful (Luckasson et al., 2002; Schalock et al., 2010).

Although the reason for the deficit-based classification reinstatement was not stated in either the 2002 or 2010 AAIDD manuals, it is not unreasonable to suspect that one reason that the AAIDD continued to support classification by level of impairment was simple inertia. This deficit level approach to classification is widely used and accepted. Until a viable alternative 
emerges that people will actually choose to use, the classification by deficit level approach is unlikely to be discarded. The authors of the AAIDD 1992 manual attempted to offer the field of ID/DD an alternative when they proposed classifying people by intensity of support needs (Luckasson et al., 1992).

Support needs classification. The many controversies surrounding the 1992 AAIDD manual were discussed previously. Two of the most contentious aspects were (a) measuring $\mathrm{AB}$ by 10 separate adaptive skill areas, and (b) eliminating classification by level of deficit according to IQ score. At first glance these two controversies seemed unconnected, but there was an important linkage. Critics charged that distinctions between these 10 skill areas were arbitrary; there was no empirical or conceptual basis for claiming the 10 areas were independent of one another (Greenspan, 1997; MacMillan et al., 1993; Thompson, McGrew, \& Bruininks, 1999).

Had the 10 adaptive skill areas only pertained to measuring $\mathrm{AB}$, this aspect of the manual would not have had a bearing on classification issues. The authors of the 1992 AAIDD manual, however, called for classification by intensity of support needs to displace classification by deficit level. Moreover, classification by support needs was to be accomplished through a process where a planning team considered the 10 areas of adaptive skills, and proceeded to rate each adaptive skill area using the following 4-point metric that reflected the intensity of support a person required from less intense to more intense (i.e., Intermittent, Limited, Extensive, or Pervasive or the ILEP supports classification approach). The four ratings on this metric were not operationally defined, and there were limited guidelines provided regarding what a planning team should consider to arrive at supports intensity ratings.

The authors of the AAIDD's 1992 manual made it clear that different people with ID may require different intensities of supports across the 10 adaptive skill areas and that the 10 areas (with ratings and narrative notes) would create a profile of support needs (Luckasson et 
al., 1992). It was not clear how a team would arrive at an overall level of support needs rating other than through considering the ratings for each of the 10 areas and coming to a consensus for a global rating at the end. Critics pointed out that because there were no reliable and valid assessment scales to measure support needs, the 1992 support needs classification approach lacked psychometric reliability and validity (Greenspan, 1997; MacMillan et al., 1993).

Two years following publication of the 1992 manual, the American Psychiatric Association (APA; 1994) chose to stick with the traditional 4-level deficit classification approach and did not mention support needs intensity classification as an option when they published their newest edition of the Diagnostic and Statistical Manual of Mental Disorders $(D S M-I V)$. The APA had traditionally followed the AAIDD's lead when establishing ID definitional and classification guidelines for the DSMs. Polloway, Smith, Chamberlain, Denning, and Smith (1999) presented data showing very few jurisdictions had adopted the AAIDD's new support needs classification approach. In response to these concerns, AAIDD President Robert Schalock formed the Support Needs Assessment Task Force to develop a standardized procedure to assess and measure the support needs of people with ID/DD (Wehmeyer et al., 2009). The work of this task force resulted in publication of the Supports Intensity Scale (SIS; Thompson et al., 2004a; 2004b).

The detailed description of the ILEP approach to classification by support needs was dropped from the AAIDD's 2002 manual. The 2002 manual authors, however, continued to maintain that a classification approach that furthered understanding of the support needs of people with ID would be preferable to the traditional deficit-based approach for purposes of planning. In contrast to the 1992 manual, the 2002 manual indicated that a variety of classification approaches might be useful depending on different purposes. For specific types of research, the deficit-based approach might be appropriate. For medical research and medical treatments, an etiological approach might be the correct one to use. Although support needs 
assessment and classification was no longer connected to the 10 adaptive skill areas from the 1992 manual, the importance of assessing support needs to guide the planning and delivering of personalized supports continued to be emphasized (Luckasson et al., 2002).

As mentioned previously, the AAIDD eventually published a psychometrically reliable and valid support needs assessment scale, the SIS (Thompson et al., 2004a). The SIS had three sections. Part 1was the Support Needs Scale, which measured support needs in relation to six subscales that included 49 items associated with different life activities. For each item, three 5point rating scales were used to measure three dimensions of support needs (Frequency - how often support is needed; Time - how much time is needed to provide support; and Type - the nature and intrusiveness of support). Part 2 was the Supplemental Protection and Advocacy Scale, which provided measures of support needs in Protection \& Advocacy (P\&A) activities. The eight items in the P\&A supplemental scale used the exact same 5-point rating scales (i.e., Frequency, Time, and Type) as the six subscale in Part 1. Part 3 listed over medical conditions and behavioral problems, and required rating the intensity of supports that was needed to address the medical or behavioral concern using a 3-point Likert Type Scale.

A completed SIS assessment generates a composite (i.e., overall) norm-referenced, standard score called the Support Needs Index (SNI) score. This score indicates a person's relative intensity of support needs in relation to a standardization sample of adults with ID/DD ages 16-64. In addition to the SNI score, the SIS provides standard scores for each of the six subscales (i.e., Home Living, Community Living, Lifelong Learning, Employment, Health \& Safety, and Social Activities). The six subscale scores provide a profile of support needs.

Despite having items (a) related to life activities and (b) rated on the same metric as the six subscales in Part 1, completing the P\&A supplemental scale (Part 2) does not generate standard scores. Thompson et al. (in press) report that the P\&A Scale was originally included in Part 1, but due to only fair inter-rater reliability as reported by Clay-Adkins (2004), the P\&A 
scale was pulled out of Part 1. It was left in the SIS, however, because other psychometric indicators suggested it was a meaningful measure of support needs, and the items on the P\&A scale had content validity based on Q-Sort and other expert reviews. That is, the authors determined the items in the P\&A scale were important for users of the SIS to consider even though standard scores were not produced.

Thompson et al. (in press) have analyzed over 100,000 cases from the SIS online data collection system, and conducted rigorous inter-rater studies where the influence of different interviewers and respondents were investigated. From these analyses, there is strong psychometric support for including the P\&A Scale as a subscale in the standardized portion of the SIS when the SIS is revised. Thompson and his colleagues conclude that generating standard scores from the P\&A subscale will improve the tool's quality by enhancing the profile of support needs produced by the SIS as well as adding greater substance to the SNI score.

Regarding classification by support needs, the SIS authors indicated that although arbitrary classification groupings could be generated based on SIS scores, "at this point, there is no empirical basis for using four levels as opposed to fewer or more" (Thompson et al., 2004b, p. 43). They proceeded to discuss, hypothetically, how deviation scores could be used to establish classification levels. Such an approach, however, would have the same technical concerns as classifying by IQ scores (i.e., imposing categories on a continuous measure causes information to be lost and, therefore, offers no real advantage to the continuous measure). The SIS authors also discussed how funding patterns might be analyzed by comparing individual funding rates to SIS scores. Assuming a funding system was fundamentally fair, the authors showed how it would be possible to predict funding levels and ranges based on SIS scores.

\section{Purpose of Current Study}

Despite the early work reported in the SIS Users Manual (Thompson et al., 2004b) prior to this study, no researchers analyzed SIS aggregate data to empirically identify possible 
subgroups or clusters of people with ID based on support needs. This study filled this void in the professional literature through the application of cluster analysis procedures. Cluster analysis of SIS data, as well as other data from statewide ID/DD population (e.g., information on residential settings), was used to examine the extent of empirically supported subgroups within the ID/DD population that shared similar support needs characteristics.

\section{Understanding Supports and Support Needs}

\section{The Supports Paradigm}

Pledger (2003) suggested that there were two basic ways to understand disability: the medical model where disability is understood in terms of functional loss and impairment, and the social-ecological model where disability is understood as a mismatch between personal competence and environmental demands. The implication of a medical understanding for those involved in providing human services to people with disabilities is to try and fix the person. Increased personal independence (i.e., being less reliant on others for assistance) is valued. A human services mission that is consistent with a medical understanding of ID/DD involves pursuing either a complete cure (i.e., complete independence) or a partial cure (i.e., less dependence; Thompson \& Viriyangkura, 2013).

In contrast, human services to people with disabilities that are based on a socialecological understanding of disability do not focus efforts on curing a person. Rather, what needs to be "cured" is the context in which the person functions (i.e., fixing the mismatch between the person and the environment). The focus of human services is on arranging personalized supports that effectively bridge the person/environment gap. Effective supports (and effective human services) result in the person with ID/DD more fully participating in the same types of settings and activities that are accessed and valued by the general population. The above statement does not mean that teaching new skills to a person is inconsistent with social-ecological understanding of disability. Teaching can be a form of support if the skills 
being taught are useful in settings and activities in which the individual desires to participate. The goal of teaching new skills, however, is not to change people so that they have less of a defect, but rather to empower people to function more successfully and participate more fully in their environments and activities (Thompson \& Viriyangkura, 2013).

The phrase supports paradigm has been used by several authors over the past 20 years (see Butterworth, 2002; Claes, Van Hove, Vandevelde, van Loon, \& Schalock, 2010; Roberts, Becker, \& Seay, 1997; Thompson \& Viriyangkura, 2013) to refer to the shifting missions and activities of human services systems and organizations. In the past, organizations devoted time, energy, and resources toward "caring for or improving people" in some way, shape, or form. The supports paradigm moves the focus from care and increasing personal achievement, to investing time, energy, and resources in identifying people's support needs and matching people with supports that enhance their quality of life. Although this paradigmatic shift has certainly not been as dramatic as those that occur in the hard sciences (as discussed by Kuhn [1962]), a cursory examination of the mission statements of ID/DD human services organizations provides evidence that "care" and "rehabilitation" language has slowly been abandoned in favor of language regarding individualized supports that promote full participation in community life, valued social roles, personal satisfaction, and improved personal outcomes (see Council on Quality and Leadership, 2012).

\section{Supports and Support Needs}

Because the supports paradigm requires a different focus, many of the medical model constructs associated with a "care paradigm" (e.g., Mild ID) are less relevant. New, more relevant, constructs (e.g., support needs) must be clearly defined. Schalock et al. (2010) indicated that supports are "resources and strategies that aim to promote the development, education, interests, and personal well-being of a person and that enhance individual functioning” (p. 224). Although they mention that professional services are one type of support, 
supports can be just about anything because supports are evidenced by personal outcomes. Therefore, something as specific as a "service dog" is a support if it promotes positive outcomes (e.g., more well-being, better daily functioning). But, the service dog is not a support if it doesn't promote positive outcomes or makes personal outcomes worse (e.g., poor health outcomes would result if someone were allergic to the dog). Supports can be people (e.g., a teacher, a job coach, or a city bus driver who reminds someone that this is his or her stop) or technologies (e.g., an Augmentative and Alternative Communication device, a visual schedule, or an adapted keyboard; Thompson \& Viriyangkura, 2013). There are some types of traditional services funded through public money that are intended to serve as useful supports for people with ID/DD but are seen by critics as not actually providing support because there is limited evidence that personal outcomes are enhanced. For example, Cimera (2011) concluded that sheltered workshops did not enhance employment outcomes for people with ID.

Whereas supports are external to a person and serve to enhance personal outcomes, support needs are internal to a person and have been defined as "a psychological construct referring to the pattern and intensity of supports necessary for a person to participate in activities linked with normative human functioning" (Schalock et al., 2010, p. 224). Like other psychological constructs that are internal to a person (e.g., shyness, motivation, intelligence), the intensity of a person's support needs must be inferred because it is not directly observable. Specific aspects of a person's support needs are certainly observable (e.g., Does a person need support to take medicine?), but a person's overall intensity of support needs in relation to others must be inferred from consideration of support that a person needs to function successfully in a variety of life activities. Leading researchers have suggested that support needs are a multidimensional construct; the dimensions of support needs, however, are highly interrelated (Schalock et al., 2010; Thompson et al., 2009; Thompson \& Viriyangkura, 2013; Wehmeyer et al., 2009). 
Because every human being has support needs, it is critical to clarify how extraordinary support needs that characterize people with ID/DD are different from the everyday (i.e., nonextraordinary) support needs of others in society. The publication of reliable and valid measures of people's (with ID/DD) support needs (see Hennike, 2002; Hennike, Myers, Realon, \& Thompson, 2006; Riches, Parmenter, Llewellyn, Hindmarsh, \& Chan, 2009; Thompson et al., 2004a; Thompson et al., 2012) has been underway for little more than a decade. Nobody has suggested that any current assessment is suitable for diagnostic purposes (i.e., determining who has support needs that are sufficiently extraordinary to warrant a clinical diagnosis of ID). In

fact, no current support needs assessment scale has been normed on the general population, and the SIS is the only scale that has been normed on a population of people who were diagnosed with ID or a related condition. Because there is evidence, however, that support needs assessment scales provide a reliable and valid way to differentiate people with ID/DD in regard to relative intensity of support needs, the factor structure of the SIS and other support needs measurements need to be investigated to identify dimensions of support needs that have empirical support.

\section{Research on the Factor Structure of the SIS}

The SIS is the only support needs scale where data from the items have been used to identify underlying dimensions of supports (i.e., latent factors). In separate factor analytic studies, Verdugo, Arias, Ibanez, and Gomez (2006) and Kuppens et al. (2010) investigated a 6factor structure based on the six subscales of the SIS (i.e., having six subscales implied a 6factor structure). Both groups of researchers concluded that their data supported this model. Interestingly, they did not investigate a hierarchical structure, which is implied by the SIS because there is a composite score (i.e., the SNI score) reflecting overall support needs.

Bossaert et al. (2009) completed factor analysis using SIS scores from a population with diverse disability characteristics and reported that their data supported a 4-factor model. 
Bossaert et al., however, proposed a shorter version (22 items) of the SIS and used this subset of items to identify following four dimensions of support needs: Personal and Social, Community Living, Daily Living, and Work. In an exploratory factor analytic study where SIS subscale scores (prepublication edition) and $\mathrm{AB}$ scores were included in the same data set, Harries, Guscia, Kirby, Nettelbeck, and Taplin (2005) reported that their data were best explained by a single factor.

All of the above studies shared one potential limitation. Namely, due to the fact that support needs assessment scales were relatively new (these have been around for little more than a decade), it is highly likely that all of the potential items that reliably and validly measure support needs have not yet been identified. Therefore, the prior models are all limited in their comprehensiveness if it is true that important measures of support needs have not yet been identified.

Recent research revealed that the eight items on the P\&A scale provide both reliable and valid measures of support needs. Thompson et al. (in press) reached this conclusion by conducting three inter-rater studies (i.e., inter-interviewer ${ }^{2}$, inter-respondent ${ }^{3}$, mixed inter-rater $^{4}$ ) where reliability coefficients on the P\&A scale were comparable to reliability coefficients reported for the six SIS subscales (as reported by Thompson, Tassé, \& McLaughlin, 2008). Moreover, through analyzing over 100,000 assessments included in the SIS-online data base, the psychometric properties of the P\&A scale were quite robust in regard to internal consistency reliability as well as construct validity (as measured by correlations of the P\&A scale the other SIS subscales and age). The P\&A scale will be included as a subscale in the next version of the SIS (J. R. Thompson, personal communication, November 1, 2013).

\footnotetext{
${ }^{2}$ Different interviewers with same respondent

${ }^{3}$ Same interviewer with different respondents

${ }^{4}$ Different interviewers with different respondents
} 


\section{Purpose of Current Study}

The research on the factor structure of the SIS and the construct of support needs warrants further investigation. First, the extent of empirical support for the latent factor models of the support needs of people with ID/DD that have been proposed needs to be investigated. Also, factor structures need to be investigated using as many valid measures of support needs as possible. This study filled this void in the professional literature through applying confirmatory factor analytic procedures to data from the six subscales of the SIS and the P\&A scale that were collected from a statewide ID/DD population.

\section{Research Questions}

This study contributed to a research-based understanding of support needs of people with ID/DD by (a) investigating classification structures according to commonalities in support needs, personal circumstances, and life conditions, and (b) investigating factor structures of the support needs construct based on support needs assessment data. The following research questions guided data analyses:

1. What multiple grouping model, based on differing support needs, is generated from SIS data collected on a statewide sample of people with ID/DD using cluster analysis?

2. Using confirmatory factor analysis, which structural model of support needs is best supported by data from a statewide sample of people with ID/DD?

\section{Definition of Terms}

For the purpose of this study, the following terms are defined:

Intellectual and related developmental disabilities (ID/DD) - a population of citizens receiving services from state agencies that are partially funded through the DD Act, for whom practicing professionals work with on a daily basis whose primary disability is not related to a sensory disability (i.e., vision, hearing) or to mental health issues. People with ID/DD are in need of extraordinary supports to participate in life activities and experience life conditions that 
are valued by the vast majority of others in society (Braddock et al., 2008; Schalock et al., 2010; Thompson \& Wehmeyer, 2008).

Supports - "resources and strategies that aim to promote the development, education, interests, and personal well-being of a person and that enhance individual functioning" (Luckasson et al., 2002, p. 151).

Support needs - "a psychological construct referring to the pattern and intensity of supports necessary for a person to participate in activities linked with normative human functioning" (Thompson et al., 2009, p.135).

Cluster analysis - a statistical technique for "exploring data sets to assess whether or not they can be summarized meaningfully in terms of a relatively small number of groups or clusters of objects or individuals which resemble each other and which are different in some respects from individuals in other clusters" (Everitt, Landau, Leese, \& Stahl, 2011, p. 13).

Factor analysis - "a statistical procedure of uncovering a (usually) smaller number of latent variables by studying the covariation among a set of observed variables" (Long, 1983, p. 11).

\section{Assumptions}

There are two primary assumptions associated with this study. First, the characteristics of people with ID/DD served by the Colorado Department of Human Services are similar to people with ID/DD served by other jurisdictions. Second, the demographic, support needs, personal competency, and life status data collected by the State of Colorado are accurate.

\section{Limitations of the Study}

Although this data set is comprehensive regarding demographic variables, support needs assessment information, personal competency data, and life status data, no pretense was made that all potentially relevant data were included in the data set. Therefore, different clusters and different latent factor structures could result if additional data were available. 


\section{Chapter Summary}

There have been many approaches to naming, defining, and classifying people with ID/DD over time. This heterogeneous population of citizens has traditionally been understood primarily by their deficits. Even today, the most common means to classify people with ID/DD is by the severity of impairment based on the extent of IQ score deviation from the population mean. A social-ecological approach to understanding disability, including ID/DD, however, has been gaining acceptance in recent years. A new scale, the Supports Intensity Scale (SIS; Thompson, 2004a) was introduced that allows for a reliable and valid assessment of people's support needs. The ability to measure a construct of interest often provides opportunities for progress in a field (Thompson et al., 2009), and the field of ID/DD is no exception. This study investigated SIS data, along with other data related to personal characteristics and life circumstances, from a statewide population of people with ID/DD. Cluster analysis was applied to investigate ways in which people with ID/DD could be classified according to support needs commonalities. Factor analysis of data was undertaken to investigate competing latent factor models of support needs. 


\section{CHAPTER II}

\section{REVIEW OF RELATED LITERATURE}

This chapter is presented in five parts. History of Intellectual Disability (ID) Conceptualization summarizes the evolution of conceptualization of ID and contributions of historical figures in the field. Classification in Intellectual and Related Developmental Disabilities (ID/DD) summarizes historical and contemporary approaches to classifying people with ID/DD and purposes served by classification. Supports and Support Needs presents the rationale underlying understanding people with ID/DD by their support needs and the evolution of support needs assessments. Supports Intensity Scale (SIS) presents basic information, strengths and criticisms, and literature and studies conducted on this scale. Factor Analysis and Cluster Analysis in Special Education discusses how factor and cluster analytic methods have been utilized in the field of special education and related fields.

Wehmeyer (2013) pointed out that it is impossible to write about how the construct of ID has been understood over time without using language applicable to a particular era. Therefore, certain historical terms that are now highly offensive (e.g., idiot, feebleminded, moron, mental retardation) are used in this chapter. It would not only be wrong to misquote historical sources, but to a certain extent the archaic terminology is helpful in understanding how people with ID were perceived in earlier eras.

\section{History of ID Conceptualization}

\section{Earliest Work Prior to 1800}

ID has existed throughout human history. Records of hydrocephaly and microcephaly date back to the age of Mesopotamia (1700-560 BC; Scheerenberger, 1983). People with ID, 
however, were not explicitly distinguished from people with other disabilities (e.g., physical disabilities, sensory impairments) until the $1800 \mathrm{~s}$. The terms natural fools or idiots were used for "people whose appearance, characteristics, speech and actions were somewhat outside the accepted norm" (Brown \& Radford, 2007, p. 21). Societal attitudes toward people with ID prior to the 1800 s varied widely. For the most part, however, attitudes were not positive. For example, "Monsterology" in 2800 BC categorized people with disabilities into three subclasses: excess in size or number, smallness in size or appendices, and hermaphrodism (Scheerenberger, 1983). Religious doctrine related disabilities to sin, the devil, and punishments (MacMillan, 1977; 1982). Many people were used for amusement (e.g., as buffoons and jesters; Mutua, Siders, \& Bakken, 2011; Thompson \& Wehmeyer, 2008).

Scholarly attempts to measure intelligence and identify people with ID date back to the 1500s. A test of intellectual ability was created by Sir Anthony Fitzherbert (1470-1538) and published in a journal, Natura Brevium, in 1534. According to Fitzherbert, a sot (also called as a simpleton or a natural idiot) was someone who could not “... account or number twenty pence, nor [could] tell who was his father or mother, nor how old he [was], ... hath no understanding of reason what shall be for his profit nor what for his loss" (as cited in MacMillan, 1982, pp. 31-32).

In the $17^{\text {th }}$ and $18^{\text {th }}$ centuries, scientific knowledge and understanding of ID increased among physicians and lecturers. Notable progress in that period were naming regions of the brain, brain dissection to accurately identify the cause of hydrocephaly, and linking ID to repeated seizures or epilepsy (Mutua et al., 2011). The 1700 s to 1800 s was the period of scientific and humanitarian progress (MacMillan, 1982). Medical professionals during this time started associating medical conditions with ID (e.g., meningitis, hydrocephaly, epilepsy).

Nevertheless, superstitious beliefs from earlier eras remained. For example, many believed that ID was caused by punishment for sin to madness due to lunar cycles.

John Locke and Jean Jacques Rousseau from the 1600s and 1700s paved the way for 
future contribution from the fields of psychology and special education. John Locke (1632-1704) was an English philosopher and physician who developed theories especially relevant to the training and treatment of people with ID. He proposed that a person's mind at birth was a blank slate and people learned through experience derived from sensory perception. The most wellknown work of Locke in the field of ID was distinguishing between "idiots" who had severe difficulty learning and processing information (ID) and "lunatics" who severely misperceived reality (Mutua et al., 2011).

Jean Jacques Rousseau (1712-1778) was a Swiss-French moralist and philosopher who affirmed Locke's theory that the senses were central to the formation of the mind (Mutua et al., 2011). Rousseau's thoughts on sensory training and other theories influenced special education pioneers like Jean Marc Gaspard Itard, Edouard Seguin, and others. Unfortunately, prior to 1800 people with ID were mostly viewed as different, hopeless, and uneducable. Established on the belief of incurability, asylums for people with ID were built to "protect" society, not to help people with ID gain more skills to go back to their families and communities (Brown \& Radford, 2007; Thompson \& Wehmeyer, 2008).

\section{A Professional Field Emerges: 1800 - 1899}

Jean Marc Gaspard Itard (1774-1838) profoundly impacted the emerging field of ID during the $1800 \mathrm{~s}$. He was greatly admired for his work with a child named Victor, also known as the Wild Boy from Aveyron. Victor was diagnosed as an "incurable idiot" when Itard met him. Itard created the first systematic, individualized educational program (i.e., intense stimulation and environmental interaction) to teach Victor to speak, care for himself, and recognize words and letters (MacMillan, 1977; 1982). Although Victor improved socially, academically, and functionally (i.e., he mastered a number of independent living skills), Itard considered his work with Victor a failure because Victor was not "cured" at the conclusion of his education. For example, Victor was still nonverbal. Despite considering himself a failure, Itard's work inspired 
others (e.g., Maria Montessori, Edouard Seguin) to believe that people with ID could learn (Gearheart \& Litton, 1975).

Edouard Seguin (1812-1880), a physician and educator from France and a student of Itard, hypothesized that ID was related to a lack of educational opportunities. He believed appropriate treatment (i.e., his physiological approach to education) would overcome the disability (Gearheart \& Litton, 1975). Using this hypothesis, Seguin taught a student with ID to speak, write, and count within 18 months (Thompson \& Wehmeyer, 2008). He also wrote the first textbooks about teaching techniques for ID such as The Moral Treatment, Hygiene, and Education of Idiots and Other Backward Children published in 1846 and Idiocy and Its Treatment by the Physiological Method in 1866 (Gearheart \& Litton, 1975; MacMillan, 1977; Scheerenberger, 1983; Thompson \& Wehmeyer, 2008). Seguin also played a significant role by calling together fellow medical doctors whose practices were focused on people with ID to share knowledge and improve services. The group was first named as the Association of Medical Officers of Hospitals for the Insane and went through several name changes. It is currently known as the American Association on Intellectual and Developmental Disabilities (AAIDD). The AAIDD was the first professional organization in the field and Seguin was its first president (Gearheart \& Litton, 1975).

Later in the 1800 s, people's understanding of ID and services for this population dramatically changed. Residential training schools became large institutions and were exclusively managed by medical professionals. Consequently, names of the places were changed (e.g., schools to hospitals, living units to wards), disabilities became categories of diseases, and the education-oriented focus of the first half of the 1800 s was altered to a medical orientation by the end of the century. Perhaps the most significant change from the early 1800 s to the late 1800 s is that ID was conceptualized as a lifelong condition. Leaders in the field became discouraged with the educative approach and viewed ID as a trait that was not curable. People with ID were 
considered to be in need of long-term care, not education (Thompson \& Wehmeyer, 2008). Moreover, people with ID “were no longer viewed as 'unfortunates' or 'innocents' who, with proper training, could fill a positive role in the home or community... they had become undesirable... the social parasites" (Scheerenberger, 1983, p. 116). Consequently, attitudes of professionals (e.g., medical, social work, psychology) changed from "the social problem of ID" to "ID as the social problem" (e.g., prostitution, poverty, crime; Scheerenberger, 1983; Thompson \& Wehmeyer, 2008).

\section{Institutionalization and Scientific Application: 1900 - 1949}

At the beginning of the 1900 s, ID was loosely defined because there was no widely accepted formal definition and no systematic means of ID classification. Alfred F. Tredgold proposed an ID definition in 1908 that was widely acknowledged. According to Tredgold, ID was "a state of mental defect from birth, or from an early age, due to incomplete cerebral development, in consequence of which the person affected is unable to perform his duties as a member of society in the position of life to which he is born" (as cited in Luckasson et al., 2002, p. 20).

The eugenics movement started in the late 1800s and became very influential during the early 1900s. The term eugenics referred to the "hereditary improvement of the human race by controlled or selective breeding" (Thompson \& Wehmeyer, 2008, p. 32). Eugenicists believed that people with ID were "poor genetic stock" and promoted the passage of involuntary sterilization laws. Residential institutions became less like hospitals and more like prisons to protect society from the menace of the feebleminded (Putnam et al., 1913). The educative and habilitative efforts were abandoned in favor of practices promoting segregation and social control. Prevention was carried out in terms of prolonged institutionalization, prohibition of marriage, and desexualization (Scheerenberger, 1983). Although eugenics was later proven to be a pseudoscience (Arnold, Riches, \& Stancliffe, 2011), it brought much tragedy into the lives of 
people with ID and their families. Support for it did not come to an end until after the horrors of the Holocaust were exposed (Scheerenberger, 1983).

Despite the eugenics movement, there were some positive advances in the early 1900s. The first public classrooms for students with ID were started. Medical advancements were evident (e.g., discovery of Tuberous Sclerosis, Tay-Sachs syndrome, cerebral palsy; MacMillan, 1982). Also during the first half of the $20^{\text {th }}$ century, IQ tests became the primary means to diagnose ID. In Europe, Alfred Binet and Theodore Simon developed a systematically administered intelligence test that the American, Henry Goddard, later translated to English. Binet's Intelligence Test was originally created in 1905 to identify Parisian students who were at risk of not making adequate academic gains (Bryant, 1997; Jacobson \& Mulick, 1996). The assessment was different from previous measurement tools in that it provided evidence of individual differences in human performance. Binet and Simon had school-aged children carry out relatively complex tasks rather than simple sensory or motor tasks. Their test was able to reliably predict future academic and vocational performance of children, and it was perceived to be a more objective way to identify children with ID than semi-structured physician interviews (Routh, 1996).

By 1916, Lewis Terman (1877-1956) and his colleagues at Stanford University had revised and collected normative data on Binet's test. The scale was renamed the Stanford-Binet Intelligence Scale and revised versions of it continue to be used today. The IQ test has been used by educators to measure intellectual abilities of students, and often separate special education classrooms become the default placement for students with low IQ scores (Gearheart \& Litton, 1975; MacMillan, 1982). Terman categorized ID by IQ score in 1916, where he proposed five levels: Dullness (IQ 80-89), Borderline ID (IQ 70-79), Moron (IQ 50-69), Imbecile (IQ 25-49), and Idiot (IQ < 25). Therefore, IQ testing and scores were used to not only divide the population by people with and without ID, but also to categorize individuals with ID into groups that were 
supposedly based on the amount of raw mental firepower (or lack thereof) people possessed.

Tredgold's revised ID definition in 1937 was an important contribution because it shifted the focus to "performance" rather than only innate ability. He posited that "mental deficiency is a state of incomplete mental development of such a kind and degree that the individual is incapable of adapting himself to the normal environment of his fellows in such a way to maintain existence independently of supervision, control, or external support" (as cited in Luckasson et al., 2002, p. 20). In 1941, Edgar Doll suggested a definition of ID that put even less emphasis on IQ scores and more weight on deficits in social competence (as cited in Luckasson et al., 2002). Although, during the 1940s and 1950s, the conceptualization of ID was firmly grounded in a deficits model (Bach, 2007) and considered to be incurable (Luckasson et al., 2002; Nihira, 1999), there definitely was a movement underway to conceptualize ID and understand people with ID in ways other than an IQ score as a proxy for mental prowess.

\section{The Parent Movement and Deinstitutionalization: 1950 - 1980}

Prior to the $1950 \mathrm{~s}$, having children with disabilities, especially ID, was typically considered to be a tragic family event. Parents of children with disabilities were encouraged to "allow them to die" (Groce, 1996, p. 7) or to institutionalize their children right after birth (and in some cases tell others that their babies had died). A number of parents, however, began questioning the benefits of institutionalizing their children. Many parents refused to send their children to institutions and openly went against the advice of medical professionals and societal conventions. When their children reached school age, those parents demanded appropriate public education for their children, and the concept of mainstreaming was discussed. During the 1950s, the parent movement became a powerful force for advocacy, and services for children and adults in local communities became established and grew considerably (see Wehmeyer \& Schalock, 2013).

The baby boom and parent movement following World War II resulted in a renewed 
emphasis on education and vocational training. The IQ test became increasingly viewed as an assessment tool with limited usefulness, and increased emphasis was placed on assessing what skills a person possessed. The construct of adaptive behavior (AB) was formally included in the 1959 and 1961 AAIDD definitional manuals, and a diagnosis required measuring $\mathrm{AB}$ in addition to measuring intellectual abilities (IQ). The major policy focus of the 1960s was on deinstitutionalization (i.e., moving people out of institutions and preventing new admissions; Nihira, 1999; Schalock et al., 2010; Thompson, McGrew, \& Bruininks, 1999). The concepts of deinstitutionalization, normalization, and mainstreaming reinforced attention to $\mathrm{AB}$ because the emphasis was placed on teaching skills needed for community living.

$\mathrm{AB}$ is currently defined as "the collection of conceptual, social, and practical skills that have been learned and are performed by people in their everyday lives" (Schalock et al., 2010, p. 43). Assessing $\mathrm{AB}$ was intended to bring a significant change to the field. It was projected that individuals with low IQ scores who performed well outside of the classroom would no longer be misdiagnosed with ID (Boan \& Harrison, 1997; Nihira, 1999), and over 200 AB scales were developed over four decades (Schalock, 1999). In actuality, however, the introduction of AB into diagnostic processes did very little to reduce the prevalence of ID or diminish concerns about misdiagnosis. As with IQ tests, $\mathrm{AB}$ scales also had critics. Critics charged that $\mathrm{AB}$ information was vague, invalid for classification purposes, and of limited use at school (Nihira, 1999). Also, no single scale covered all dimensions. Thompson, McGrew, and Bruininks' (1999) review of 31 factor analytic studies on AB showed, "no single adaptive-maladaptive behavior assessment instrument completely [measured] the entire range of adaptive and maladaptive behavior dimensions" (p. 28). For instance, the Comprehensive Test of Adaptive Behavior (CTAB) only included items measuring personal independence, and did not include items focusing on personal responsibility, cognitive/academic functioning, vocational and community adjustment, and so on.

By the late 1960s, exposés of shockingly poor conditions of institutions were released in 
the popular media, and these fueled outrage and advocacy for public policies promoting community integration (see Blatt \& Kaplan, 1966). The principle of normalization became a unifying philosophy for work in the field. Nirje (1969) encouraged society to provide "normal" everyday life patterns and conditions to people with ID. In addition to broadening people's experience and increasing their opportunities to participate and contribute to society, opportunities to experience normalized lives reduced the stigmatization of ID. Wolfensberger (1983) later expanded the concept by introducing the term social role valorization. He explained that people with ID needed opportunities to adopt valued social roles (e.g., employee, tax payer, home owner, church goer). The normalization concept drove the mainstreaming movement and, as a result, schools were required to place children with disabilities in the least restrictive environment (LRE) when the right to education law was passed in 1975 (i.e., PL 94-142). The LRE and the mainstreaming principle motivated education professionals and families to search for the right supports for this population in school settings, and therefore expanded the capacity of classrooms and schools to accommodate a greater diversity of learners (Drew \& Hardman, 2007).

\section{Self-Determination, Quality of Life, Inclusion, and Supports: 1980 Onward}

By the 1990s, a social-ecological conceptualization of ID appeared in several writings.

Contrasted with the deficit-based model of ID, the social-ecological model conceptualizes ID as a mismatch between personal competency and environmental demands (Thompson, Wehmeyer, \& Hughes, 2010). Based on this conceptualization, the focus of professional efforts is to eliminate barriers and provide the right supports for people to experience better personal outcomes. Internationally, a social-ecological model of disability was featured in the World Health Organization's (WHO) International Classification of Functioning, Disability, and Health (ICF) in 2001. According to the WHO, “... every human being can experience a decrement in health and thereby experience some degree of disability... ICF takes into account the social aspects of disability and does not see disability only as a 'medical' or 'biological' dysfunction" (WHO, 
2012, para. 3). Upon publication of the 1992 AAIDD manual based on a social-ecological conceptualization of ID, Thompson (2010) observed that the medical conceptualization of ID "may be coming to an end" (p. 165).

The social-ecological conceptualization of ID was prominent in the 1992, 2002, and 2010 AAIDD manuals. The definition of ID was changed from a deficit trait or a defect of a person to a state of impaired human functioning. Schalock et al. (2010) explained that human functioning included all life activities and "[encompassed] body structures and functions, personal activities, and participation, which in turn [were] influenced by one's health and environmental or contextual factors" (p. 15). Therefore, under this concept, the lives of people with ID could be enhanced if they receive individualized supports. Interestingly, although the definition changed, the three criteria for diagnosing ID (i.e., intellectual functioning, $\mathrm{AB}$, and age of onset) were retained.

The current AAIDD definition of ID is:

Intellectual disability is characterized by significant limitations both in intellectual functioning and in adaptive behavior as expressed in conceptual, social, and practical adaptive skills. This disability originates before age 18 . The following five assumptions are essential to the application of this definition:

1. Limitations in present functioning must be considered within the context of community environments typical of the individual's age peers and culture.

2. Valid assessment considers cultural and linguistic diversity as well as differences in communication, sensory, motor, and behavioral factors.

3. Within an individual, limitations often coexist with strengths.

4. An important purpose of describing limitations is to develop a profile of needed supports.

5. With appropriate personalized supports over a sustained period, the life 
functioning of the person with intellectual disability generally will improve. (Schalock et al., 2010, p. 1)

The AAIDD's 2010 manual explicitly acknowledged the incongruence between its operational definition (i.e., the deficit-based criteria for diagnosis) and constitutive definition (i.e., the social-ecological conceptualization of ID). Although the operational definition focuses on the three criteria of ID and it is often consistent with a trait or deficit-based understanding, the constitutive definition refers to ID as limitations in human functioning. Instead of differences of personal competency (e.g., intelligence, AB), people with ID are best understood as individuals who need supports (i.e., type and intensity) that the general population does not need. Therefore, individualized supports play a key role in improving human functioning of people with ID. Both the operational and constitutive definitions are useful because the operational definition helps professionals make a valid and reliable diagnosis, and the constitutive definition promotes understanding people with ID by their support needs (Schalock et al., 2010).

As a result of the social-ecological conceptualization of ID, issues of human rights, individuality, dignity, and self-determination of people with ID have continually been raised. Because independence and individualism are highly valued for all citizens in modern society, all citizens, including people with ID/DD, must have opportunities for self-determination (United Nations, 1981). According to Wehmeyer et al. (2011), self-determined behavior refers to "volitional actions that enable one to act as the primary causal agent in one's life and to maintain or improve one's quality of life" (p. 21). Promoting self-determination has become a best practice in education and transition services for people with disabilities (Wehmeyer \& Field, 2007).

According to Schalock (2004), quality of life is a principle that enhances aspects of wellbeing (e.g., emotional, material, physical well-being) of people with ID/DD. It also includes social inclusion, which does not mean just being physically included but also meaningfully 
participating in activities valued by same-aged peers. These concepts have been set as outcomes for education, health, and social service programs; therefore, service providers, administrators, and policymakers are experiencing challenges to develop supports and services that promote selfdetermination and inclusion to increase quality of life of people with ID/DD. It is important to note that these concepts and supports have a close relationship because these factors affect how supports are designed, and supports in turn impact these qualities in the lives of people with ID/DD (Claes, Van Hove, Vandevelde, van Loon, \& Schalock, 2012).

\section{Classification in ID/DD}

Regardless of the orientation to providing services to people with ID (e.g., to cure, to care, to educate), efforts to assign the population with ID to subgroups have been made since early history (e.g., the Monsterology in 2800 BC classified people with disabilities by outward physical characteristics; Scheerenberger, 1983). In the modern era with progress in science and measurements, ID classification approaches became more complex. According to Luckasson and Reeve (2001), "classifying refers to dividing into groups what has been included within the boundaries of a name or term. In classifying, one creates subgroups or clusters within the defined group, according to some established criteria" (p. 50). A classification system could be developed in various ways, based on different criteria, and for numerous purposes. This section is presented in three parts: Historical Approaches to Classifying People with ID, Purposes Served by Classification, and Classification for the Purpose of Resource Allocation.

\section{Historical Approaches to Classifying People with ID}

A number of classification systems (e.g., legal, educational, medical, behavioral) have been developed in the field of ID over time (Gearheart \& Litton, 1975). Although a combination of classification approaches have been proposed, three of the most important approaches include etiological classification, intellectual deficit classification, and support needs classification. This section presents each approach in detail. 
Etiological classification. Medical professionals dominated the field of ID prior to the 1900s. Therefore, it is not surprising that the earliest classification systems were based on medical conditions (Gearheart \& Litton, 1975). In fact, the classification system by etiology (i.e., biological cause) was the first approach in published history. Classification by etiology started with simply observing outward physical characteristics of a person. Later, due to medical advances, etiological classification systems became more complex and several disability conditions were identified or classified before or right after birth. Dr. Langdon Down proposed that ID could be classified as Congenital Idiocy (i.e., microcephaly, hydrocephaly, paralysis and epilepsy), Developmental Idiocy (due to anxiety associated with cutting teeth or puberty), or Accidental Injury (due to injury or illness; Grossman, 1983). The most famous work of Down, however, was identifying a subgroup of people with ID by physical characteristics (including the individuals with the condition currently known as Down syndrome; Harris, 2006; Scheerenberger, 1983). Today, Down syndrome can be detected before birth with prenatal genetic diagnosis (e.g., blood testing, amniocentesis, chorionic villus sampling) although accuracy as well as safety of different tests vary (see Shahbazian, Barati, Arian, \& Saadati, 2012).

Understanding etiology can lead to prevention of ID. For example, Phenylketonuria (PKU) used to be a relatively common biological cause of ID. Today, PKU can be detected right after birth (through newborn screening), and the resulting ID can be prevented (through a special diet). As a result, the number of children with ID caused by PKU enormously decreased over the past 50 years (Dykens, Hodapp, \& Finucane, 2000; MacMillan, 1982; National Institute of Health, 2000). Ways to prevent other ID causes (e.g., Fetal Alcohol Syndrome, Shaken Baby Syndrome), medical treatment for some conditions (e.g., hydrocephaly), and chromosomal studies furthering knowledge of conditions such as Down syndrome and Fragile $\mathrm{X}$ syndrome have demonstrated the importance of etiological classification in the field of ID (National Down Syndrome Congress, 2012; National Institute of Neurological Disorders and Stroke, 2012; 
Streissguth, 1997).

Although etiological classification can assist professionals in identifying and planning physical health-related services (Schalock et al., 2010), over half of ID cases have no known cause. Therefore, the classification system by etiology is not comprehensive. Most importantly, although etiological classification can be useful in medical research and medical treatments, it yields limited benefits for professionals and practitioners in other disciplines (e.g., education, psychology, social work) who need to plan and provide behavioral interventions (Durkin \& Stein, 1996).

Intellectual deficit classification. Intellectual deficit classification has been greatly utilized in the fields of special education and psychology. Intelligence refers to "a general mental ability .... [it is] not merely book learning, a narrow academic skill, or test-taking smarts. Rather, it reflects a broader and deeper capacity for comprehending our surroundings" (Schalock et al., 2010, p. 15). The extent to which people lack "intellectual firepower" determines their degree of intellectual deficit. Classifying individuals with ID by intellectual deficit can be approached in several ways, but always requires the use of intelligence tests. For example, from the 1800 s until the early 1900s, people with ID were classified as Simpleness (consistent with today's developmental delay), Backwardness (consistent with Mild ID), Imbecility (consistent with Moderate ID), and Idiocy (consistent with Severe and Profound ID) based on physician interview, which was a crude and non-standardized means of measuring intelligence (Mutua et al., 2011).

Another archaic approach was to classify people with Simulative Idiocy (who could function in daily lives fairly close to the general population), Higher-Grade Idiocy (who could go to school and function in daily lives if they received some special training), Lower-Grade Idiocy (who could learn some functional skills and live in the community if they received support), and the "Incurables" (people who should not receive education because it would be pointless). A final archaic approach involved aligning verbal communication with ID. For example, some 
posited that people with ID should be classified as Imbeciles who had mental deficiencies but could communicate verbally, Idiots who could use a few words and short sentences, Idiots who could utter monosyllables and grunts, or Idiots who completely had no language (Brown \& Radford, 2007). Although the "tests" used to place people in these categories were not standardized and were not labeled as intelligence test per se, the fact that people were categorized by degree of intellectual deficits mean that the tests were considered to be valid ways to measure human intelligence.

In the early 1900s, Binet and Simon developed the original IQ test which yielded mental age scores. The IQ test involved uniform procedure for assessment and was highly reliable. Later, the Stanford-Binet intelligence test was published with IQ scores that were standardized across age groups. The IQ score began to drive diagnosing and classifying in the field of ID. The IQ test was perceived as a valid and reliable diagnostic and classifying tool, and became the field's standard (Bach, 2007; Durkin \& Stein, 1996; Harris, 2006). Diagnosis and classification by IQ score and intellectual deficit were adopted by the AAIDD 1910 Committee on Classification of the Feebleminded. They saw IQ tests as an objective means to classify individuals with ID into groups based on severity levels of limitations or deficits. The 1910 Committee identified three tiers of people with ID: Morons (with relatively high mental age scores), Imbeciles (who had mid-range mental age scores), and Idiots (with low mental age scores; Smith \& Wehmeyer, 2012). This approach was included in what is now considered to be the first edition of the AAIDD definition and classification manual (Schalock et al., 2010). Later, from 1958 to 1973, there were five levels of ID: Borderline ID (IQ 67-83), Mild (IQ 50-66), Moderate (IQ 33-49), Severe (IQ 16-32), and Profound (below 16; Scheerenberger, 1987).

The Borderline category, which significantly contributed to the overrepresentation of poor minority children in special education, however, was discarded in 1973. In its 1973 manual, the AAIDD changed the IQ upper limit cutoff score from one to two SDs below the mean; and 
that removed the Borderline group from the field's leading ID definitional manual. Also in 1975, Public Law 94-142 (PL 94-142 or Education of All Handicapped Children Act, now the Individuals with Disabilities Education and Improvement Act or IDEA) was passed. This law mandated that students with disabilities had the right to a free and appropriate public education, and the disability category of learning disability (LD) was included under this federal law. When LD was defined as a discrepancy between IQ and academic achievement, many children with IQ scores in the Borderline range were diagnosed with LD instead of ID (Reschly et al., 2002; Thompson \& Wehmeyer, 2008; Ysseldyke et al., 2000).

Unfortunately, evidence suggests that many children who would have been diagnosed in the Borderline range in an earlier era were no longer eligible for special education services. The persistent achievement gap between socioeconomic groups and ethnic/racial groups in schools also suggests that eliminating the Borderline group from ID did not eliminate this group's need for special education and other supports in school (MacMillan, Gresham, Bocian, \& Lambros, 1998; MacMillan et al., 1993).

Over time, there have been concerns regarding the use of IQ scores in diagnosing and classifying ID. First, IQ is explicitly linked with deficit thinking (Bach, 2007) and incurability (since IQ scores are stable, ID is not curable; Luckasson et al., 2002; Nihira, 1999). Second, IQ scores provide very little direction for interventions (Thompson \& Wehmeyer, 2008). Third, because IQ scores reflect linguistic and quantitative skills (i.e., skills related to academic achievement), social intelligence is not measured by IQ tests, and social vulnerability (e.g., gullibility, exploitation) is considered to be the most important area of limitation for people with ID by many scholars (e.g., see Greenspan \& Granfield, 1992; Greenspan, Loughlin, \& Black, 2001).

One of the biggest concerns with classification by IQ deficit level is that educators have used it to guide educational placement. Also, adult service providers have used it to drive 
program placement. When placement becomes confused with intensity of support needs (i.e., more restrictive placements that provided more intensive supports and services were reserved for those with lower IQ scores), people with lower IQs are assumed to have more intense support needs and may get an intensity of service and oversight they do not want or need. On the contrary, people with higher IQs are assumed to have less intense support needs and therefore may get inadequate services, or no services at all because they are not a priority. Therefore, pigeonholing people into placements by IQ level has not been beneficial in planning and providing individualized supports and services (Snell et al., 2009).

In the educational systems, IQ score classification has led to many children receiving special education in segregated settings (e.g., special schools for students in the "severe" category). Studying in segregated environments prevents them from opportunities to have interactions with their same-aged peers without disabilities. Consequently, they lose access to appropriate models for communication, behavior, and so on (Schalock et al., 2010). In adult service areas, placement by IQ score provides equally poor opportunities. For example, in the 1980s, there was "a vocational rehabilitation tradition of screening out the so-called 'nonfeasibles' - persons who [were] 'not expected' to benefit from rehabilitation services" (Bergman, n.d., p. 3). Additionally, terms associated with deficit categories (e.g., Trainable and Totally Dependent) often result in low expectations and misguided perceptions toward children and adults with disabilities. Such a deficit-based classification approach draws attention away from people's strengths and the need for supports. Therefore, it has limited usefulness for service providers (Durkin \& Stein, 1996).

A final problem of classification by level of intellectual deficit is that IQ is a continuous measure. Reducing the IQ score into four levels causes information to be lost (Durkin \& Stein, 1996). Just as putting people into four levels of height (i.e., Very Tall, Tall, Short, and Very Short) does not communicate as complete information as could be provided by simply reporting 
their heights in inches, putting individuals with ID/DD into one of four severity levels (e.g., Mild ID) reduces the information that is communicated.

These concerns prompted the AAIDD to eliminate the deficit-based classification approach from its 1992 manual and propose a new classification approach based on intensity of needed supports (Luckasson et al., 1992). This new approach was aligned with a socialecological conceptualization of ID. The deficit-based classification, however, returned as part of a multidimensional classification approach in the two most recent manuals (Luckasson et al., 2002; Schalock et al., 2010). The authors of these manuals reasoned that ID classification has different purposes, and clinicians are faced with different classification questions (e.g., Is the person capable to be a witness? Is the person capable of parenting a child?). Therefore, classification must be linked to purpose, and classification purposes are more varied than any single approach can accommodate. Intellectual deficit classification is still an option in classifying ID.

Support needs classification. One major purpose in identifying and classifying people with ID is to develop a profile of support needs that can guide the provision of individualized supports (Schalock et al., 2010). Authors of the 1992 AAIDD manual advocated for classifying individuals with ID to reflect a social-ecological conceptualization of ID that was based on the premise that ID was best understood as a state of human functioning (Luckasson et al., 1992). The 1992 manual provided a process to identify support needs of an individual, which involved a planning team rating support needs relevant to 10 adaptive skill areas. When the ratings were completed, documentation was generated that showed a pattern of support needs as well as his or her intensity of support needs (from less intense to more intense). The documentation was based on a 4-point supports intensity metric (i.e., Intermittent, Limited, Extensive, or Pervasive and was called the ILEP supports classification approach). Although Luckasson et al. were not clear on how the ratings were to be combined to arrive an overall support needs classification, a 
summative judgment on an overall support needs category was to be made at the end of the process.

The ILEP classification approach was criticized for a number of reasons. First, critics pointed out that the 10 adaptive skill areas did not have empirical support. In other words, there was no research to support the claim that the 10 adaptive skill areas were independent of one another or reflected the totality of adaptive skill dimensions needed for participation in school and society (Greenspan, 1997; MacMillan et al., 1993; Thompson, McGrew, \& Bruininks, 1999). Second, there was no evidence that the process for arriving at supports ratings were reliable or valid (MacMillan et al., 1993). Although the 1992 manual suggested a planning team rate the level of supports intensity on a 4-point metric, it provided only limited guidelines on how a planning team should arrive at the rating. Possibly due to these criticisms, the American Psychiatric Association (APA, 1994) broke with tradition and chose to not adopt the ILEP classification approach in the new edition of their diagnostic manual (DSM-IV). Rather, they chose to continue using the 4-level deficit classification approach (i.e., Mild, Moderate, Severe, and Profound). Polloway, Smith, Chamberlain, Denning, and Smith (1999) noted that very few jurisdictions in the U.S. had adopted the AAIDD's support needs classification approach five years after being published.

Although the ILEP classification approach was mentioned in the 2002 AAIDD manual (Luckasson et al.), the process for arriving at ratings was abandoned. In the 2010 AAIDD manual (Schalock et al.), there was no mention at all of the ILEP approach. In both manuals, however, there was continued emphasis on the importance of assessing support needs to guide the planning and delivering of personalized supports. In response to the unsuccessful launching of the ILEP classification approach, the AAIDD assigned the Support Needs Assessment Task Force in 1999 to develop a uniform procedure to assess pattern and intensity of needed extraordinary supports of people with ID/DD that yielded psychometrically defensible results (Wehmeyer et al., 2009). 
The work of this task force resulted in publication of the Supports Intensity Scale (SIS) in 2004 (Thompson et al., 2004a). The SIS will be discussed in detail later in the chapter.

\section{Purposes Served by Classification}

In the 2010 AAIDD manual, Schalock et al. concluded that researchers and practitioners have different professional goals, therefore multiple classification approaches are needed to serve different purposes. They identified several purposes of classification, including (a) research, (b) provision of services and supports, (c) communication about selected characteristics of persons and their environments, and (d) funding.

The first purpose of ID classification is research. A number of studies have focused on personal competence, quality of life, and personal outcomes, all of which are influenced by individual differences such as intellectual abilities, AB, health, and support needs. Appropriate classification systems of ID assist researchers in analyzing data to identify individual differences that serve as significant outcome predictors. For example, researchers have conducted studies on people with ID classified by etiology (e.g., Baker, Seltzer, \& Greenberg, 2012), severity of

deficits (e.g., Hubert, 2011), and support needs (e.g., Copeland \& Cosbey, 2008; Riches, 2003) to identify variables associated with community adjustment, quality of life, and achievement.

The second classification purpose is to provide services and supports. The essence of the supports paradigm is that, supports and services should be individualized/personalized, based on the pattern and intensity of supports needed (Thompson \& Viriyangkura, 2013). Although supports should be individualized, it is reasonable to assume that many people will share similar support needs and therefore will need similar supports, both quantitatively and qualitatively. Classification by support needs could help identify common ways to support groups of people so that there was not a need to "reinvent the wheel" for every case. Although there is always the danger that a "group mentality" could take over and supports lose personalization, classification also has the potential to identify common support needs and strategies that are successful and can 
inform decision making in planning teams.

Classification can facilitate communication among professionals, families, and relevant parties about factors influencing human functioning. It is difficult to communicate information without classification terminology; it is how people make sense of the world (Schalock et al., 2010). Because people with ID are such a heterogeneous population, classification can be used to enhance understanding and communication about people.

The availability of public funds requires that decisions be made in regard to who in the population will receive what amount of funding. Funding decisions have been based on a number of personal and external factors over time (e.g., level of support needs, $\mathrm{AB}$ level, challenging behaviors, health status, residential setting, geographical location). A valid classification system could assist professionals and policymakers in allocating resources equitably and efficiently (Schalock et al., 2010).

\section{Classification for the Purpose of Resource Allocation}

A critical difference between publicly funded education services provided to children with ID/DD and publicly funded services provided to adults with ID/DD in the U.S. is the funding structure and legal guarantees. Children with disabilities in K-12 schools have a right to a free public education. The spirit of the law is that every child receives enough funding to get what he or she needs for an appropriate education, and therefore the "budget" for each child is individualized (in theory; Yell, 2006). For adult services (and certain services outside of education to children, for example residential services to children who are wards of the state), there are no such guarantees that funding will be aligned with individual needs. Although a discussion of all of the different funding streams and types of programs for which adults with ID/DD may be eligible is beyond the scope of this dissertation, funding approaches in the U.S. have come under increasing criticism for the past decade. Among the concerns are that congregate residential facilities have been funded on historical rates, which has discouraged 
development of more efficient, desirable, and individualized housing options. Moreover, funding rates get negotiated by state governments with service provider organizations with limited attention paid to quality of services or characteristics of people served (Stancliffe \& Lakin, 2005).

Concerns that decisions on budget spending "often appear idiosyncratic and unfair" (Kimmich et al., 2009, p. 1) over time and across geographic areas have been expressed regularly. For example, Colorado had 2,530 Supported Living Services Waiver (SW) participants in 2009. Fortune, Auerbach, Melda, Agosta, and Smith (2009) reported that, based on an analysis of people's support needs, at least 1,048 SW participants (41.5\%) received more resources than needed whereas at least 1,188 individuals $(46.9 \%)$ received too little resources. In recent years, there has been much interest in investigating empirically justifiable approaches to allocate available resources more systematically to provide appropriate supports and services to individuals with ID/DD (e.g., see Braddock et al., 2013; Kimmich et al., 2009; Pew Center on the States, 2010). An equitable and responsive resource allocation system that provides appropriate supports to individuals with ID/DD is sorely needed.

Historically, services for individuals with ID/DD and resource allocation were based on a facility approach. In the past, a large portion of federal and states' funding was put into $24 \times 7$ residential and day services (Fortune et al., 2009). With pressure from consumer and advocacy groups, services and supports were moved away from facilities and toward individualized, community-based services (Kimmich et al., 2009). Community supported living arrangements (CSLAs) not only yielded better outcomes for individuals but also affected public policy and funding approaches. CSLAs typically involve small houses for 4-8 residents with ID/DD who need extraordinary supports in daily lives that others in society do not need. In regard to funding, CSLAs required less financial support than traditional large facilities. The annual operating cost per resident in CSLAs has been below $\$ 50,000$ per resident, compared to state-operated developmental centers that spend a medium of approximately $\$ 100,000$ per resident annually 
(Illinois Department of Human Services, 2012; Fahey, Walsh, Emerson, \& Guerin, 2010).

The State of Colorado is one among a number of states (e.g., Georgia, Louisiana, Missouri, Oregon, Rhode Island, Virginia) that developed new resource allocation models by classifying a population with ID/DD into groups of people who have similar support needs. These states have used the SIS as a major assessment in measuring support needs of individuals with ID/DD and categorized these people into subgroups. For example, Colorado collected data on the SIS and other surveys on their Comprehensive Home and Community-Based Services Waiver (CW) Program. This program offers a wide variety of services to a population with ID/DD who are not living with families and includes $24 \times 7$ out-of-home residential services.

The Human Services Research Institute (HSRI) was hired by the State of Colorado to analyze data and propose a resource allocation model that helped the state achieve maximum benefits from its CW budget. The HSRI suggested classifying CW participants into six groups representing different levels of support needs based on three SIS subscale scores (A: Home Living, B: Community Living, and E: Health \& Safety, so-called ABE), Extraordinary Medical and Behavioral Support Needs. The six CW levels resulted from their pilot study are presented in Table 1 (Kimmich et al., 2009). 
Table 1

A Resource Allocation Model Proposed for Colorado CW Program

\begin{tabular}{|c|c|c|c|c|c|}
\hline CW level & $\begin{array}{l}\text { Number of } \\
\text { participants }\end{array}$ & $\begin{array}{l}\text { SIS ABE subscale } \\
\text { score }\end{array}$ & $\begin{array}{c}\text { SIS ABE } \\
\text { national percentile }\end{array}$ & SIS MS & SIS BS \\
\hline 1 & 200 & $\leq 30$ & 50 th & None & $\leq 6$ \\
\hline 2 & 151 & $\geq 31$ to $\leq 36$ & $51 \mathrm{st}$ to 75 th & None & $\leq 9$ \\
\hline 3 & 37 & $\geq 37$ & 76th or less & None & $\leq 10$ \\
\hline 4 & 35 & $\geq 12$ to $\leq 21$ & 33 rd to 60 th & None & 7 to 10 \\
\hline 5 & 30 & None & None & $\geq 8$ & None \\
\hline 6 & 68 & None & None & None & $\geq 11$ \\
\hline Total & 521 & & & & \\
\hline
\end{tabular}

Table 1 shows that participants in Level 1 needed less supports in the ABE subscales

(i.e., Home Living, Community Living, and Health \& Safety), did not need extraordinary medical supports, and had relatively lower intensity of behavioral supports. Participants in Level 2 had higher intensity of support needs in the ABE subscales, did not need extraordinary medical supports, and had relatively higher intensity of behavioral supports than Level 1. Participants in Level 3 required relatively more supports in the ABE subscales, had relatively higher B intensity of behavioral support needs, but did not need extraordinary medical supports. In Level 4, participants needed less supports in the ABE subscales, did not need extraordinary medical supports, but had relatively high intensity of behavioral supports. Participants in Level 5 needed only some extraordinary medical supports. In Level 6, participants needed high intensity of behavioral supports without other support needs.

\section{Supports and Support Needs}

When the supports paradigm emerged in the field of ID/DD in the 1990s, there were no widely accepted definitions of key terms (e.g., supports), and there were no standardized 
measures of key constructs (e.g., support needs; Thompson et al., 2002). The characterization of support needs as a "slippery construct" (p. 36) reflected the lack of clarity surrounding the construct at that time. Much progress, however, has been made during the past decade. This section presents information about supports and support needs in two parts. Measuring Support Needs Prior to Standardized Measurements summarizes approaches used in measuring support needs before formal support needs measurements were developed, and Current Support Needs Assessment Instruments describes support needs measurements in the field of ID/DD today.

\section{Measuring Support Needs Prior to Standardized Measurements}

Prior to specific tools to assess support needs, professionals relied on clinical judgments as scales and skill assessments to identify and address people's needs for support.

Clinical judgment. Biklen (1988) noted that legal entities used clinical judgment to determine support needs at the individual level, in courts (e.g., Board of Education of the Hendrick Hudson Central School District v. Rowley in 1982), and in Congress (e.g., in PL 94-142 mandated in 1975). Nevertheless, clinical judgment has limitations when not grounded in a standardized assessment. First, it is very subjective. When used by an individual, one person's "opinion" becomes the final determinant and there is no counterbalance to check the veracity of an opinion. Clinical judgment is also limited due to a lack of consistency between assessors. Moreover, clinical judgment is the purview of professionals who make all decisions without taking consumer and family interests and opinions into consideration. Lastly, clinical judgment is often influenced by several nonclinical factors (e.g., state funding policies, politics, labor interests, public prejudice toward people with disabilities; Biklen, 1988). To encourage clinical judgment integrity, the AAIDD published clinical judgment guidelines (see Schalock et al., 2010), but clinicians could freely choose to fully adopt, partially adopt, or completely ignore any guidelines.

AB assessments. Assuming a reciprocal relationship between personal competence and 
support needs, service provider agencies and educators have a long tradition of addressing needed supports by measuring $\mathrm{AB}$ skills that individuals lack and teaching $\mathrm{AB}$ skills to "fix" any deficits (AAIDD, 2013a). Although some researchers have presented data suggesting that AB skills and support needs scales are measuring the same construct (e.g., Harries et al., 2005), others presented data showing that $\mathrm{AB}$ and support needs are not identical (van Loon, Claes, Vandevelde, Van Hove, \& Schalock, 2010).

Certainly, at face value, it is evident that AB scales measure individuals' achievement or performance, and support needs assessments measure extraordinary supports people need to participate in daily life activities. Therefore, AB scales are at best an indirect measure of support needs because professionals must infer support needs based on deficits. Furthermore, AB scales do not provide explicit information about support needs (e.g., frequency, time, type; Weiss, Lunsky, Tassé, \& Durbin, 2009). Schalock et al. (2010) explained that support needs are influenced by five factors (i.e., personal competence, medical support needs, behavioral support needs, types of settings, and types of life activities) but AB scales are a measure of only one factor (i.e., personal competence).

Specific skills measurements. Some professionals have used specific skills measurements to assess support needs of people with ID. For example, teachers in the area of severe/multiple disabilities have long used Ecological Inventory Exemplars to assess a student's present level of performance. Ecological Inventory Exemplars are typically a list of tasks that need to be done in each particular environment to participate fully. A student's performance on the exemplars is compared to skills he or she needs to fully participate, and the discrepancy yields instructional objectives for a student as well as support areas that must be addressed (Sobsey, 1987). Another example is Job Analysis, a systematic way to determine specific demands of a job (Clark \& Kolstoe, 1995). Job analysis checklists inform teachers of a student's vocational competencies in different areas (e.g., physical demands, communication skills), and the 
discrepancy between what is needed and what a student can do directs instructional goals as well as support arrangements. Due to the narrow focus of these types of tools, however, they do not provide an overall assessment of someone's support needs. Moreover, a number of specific skills measurements would be needed to comprehensively measure an individual's support needs.

Because the abovementioned approaches and tools were not designed to directly measure support needs, information derived from these tools was not useful in comparing the intensity of support needs of different individuals. Professionals in the field had to tolerate the drawbacks of these approaches and tools until support needs assessments were developed in the late 1990s and early 2000 s.

\section{Current Support Needs Assessment Instruments}

In the late 1990s, scholars began to put their efforts into developing tools to measure support needs to help practitioners gain adequate support needs information of individuals with ID/DD. Frequently, the ability to measure an important construct leads to progress in the field (Thompson \& Viriyangkura, 2013). For example, high blood pressure leads to heart attack. With the introduction of a blood pressure gauge to reliably and validly measure blood pressure, much progress was made in understanding and treating heart attack. In the same way, support needs measurement is important to the field of ID/DD. It is difficult to understand how to provide the right supports without a way to identify or measure support needs. Several support needs assessments were created during the past 15 years and continue to be used today. Table 2 compares these tools. 


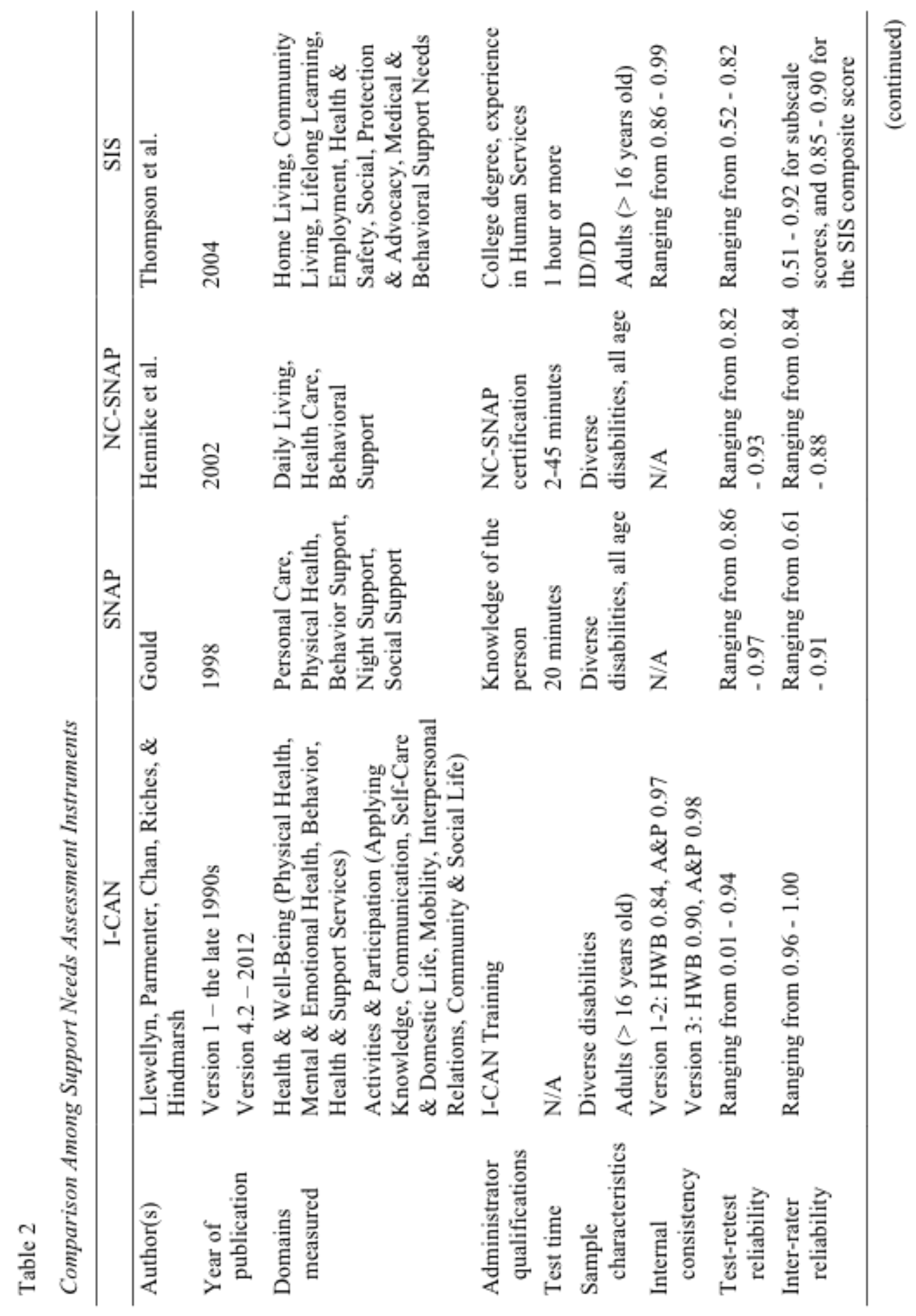




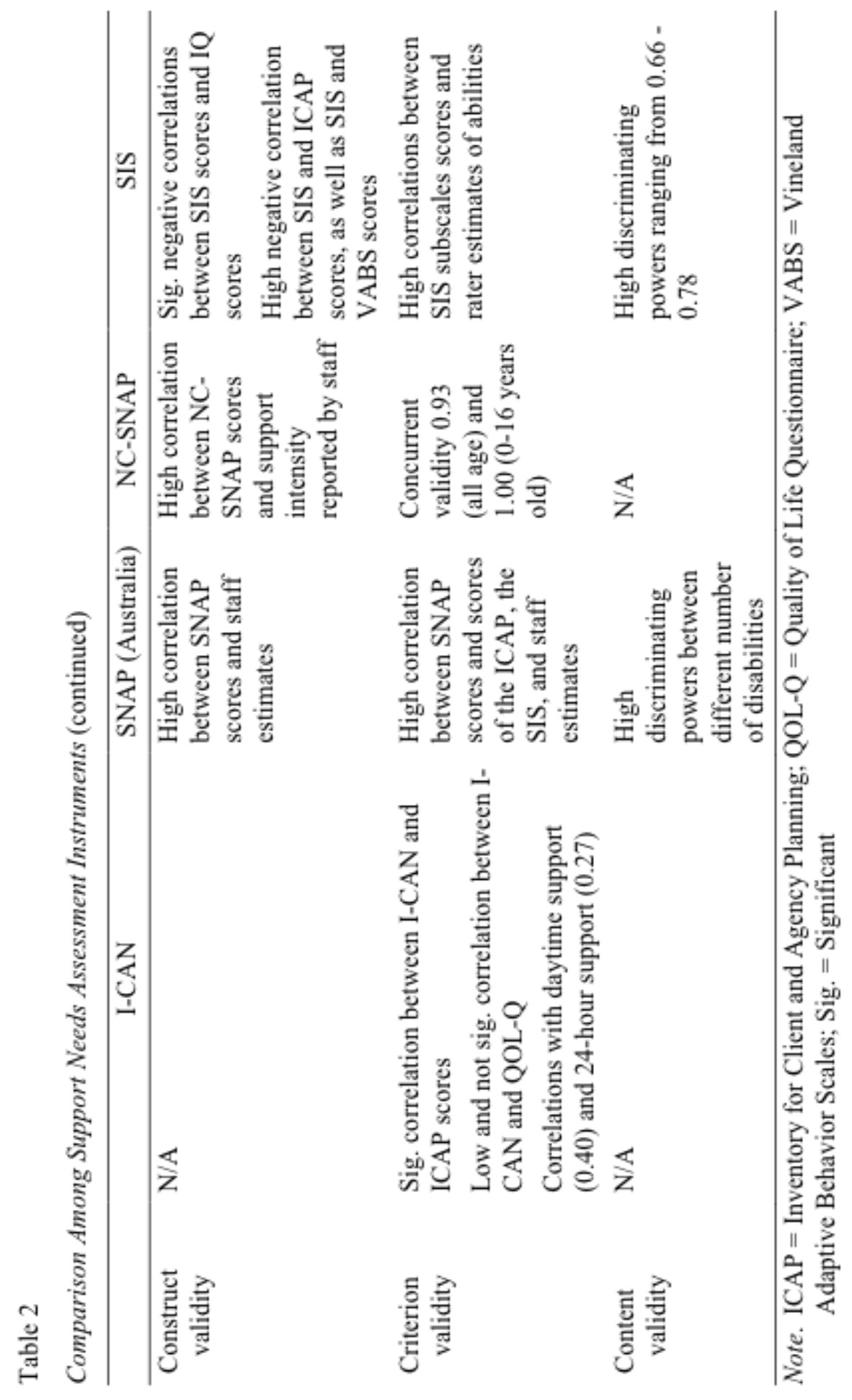


Instrument for the Classification and Assessment of Support Needs (I-CAN). The first version of the I-CAN was aimed to assess and classify the support needs of adults with different disabilities in Australia (Arnold, Riches, Parmenter, \& Stancliffe, 2009). To measure support needs of an individual, a group of respondents and a trained facilitator rates frequency and level of support needs in 10 domains (see Table 2). Information gathered from the I-CAN is to be used at both individual and systems planning levels (Centre for Disability Studies, 2012a; 2012b). The I-CAN authors reported high internal consistency, test-retest reliability, and interrater reliability coefficients. Regarding the scale's validity, correlation between the I-CAN and the Inventory for Client and Agency Planning (ICAP; an AB scale) was satisfactory, but correlation between the I-CAN and the Quality of Life Questionnaire (QOL-Q; an outcome measure) was low and not significant. When the I-CAN scores were compared with the amount of daytime support hours and 24-hour support required, the predictive validity was high (Riches, Parmenter, Llewellyn, Hindmarsh, \& Chan, 2009; see Table 2).

Service Need Assessment Profile (SNAP). The SNAP has been used to guide disability services funding in Australia. Because it was not as widely used as the other tools, the SNAP has not appeared in publications as often and has only been examined by one group of researchers (i.e., Harries et al., 2005; Productivity Commission, 2011; 2012). To complete the scale, service providers rate levels of support required by an individual in five domains of daily life activities (see Table 2). The SNAP has good test-retest reliability and inter-rater reliability (Guscia, Harries, Kirby, Nettelbeck, \& Taplin, 2005) as well as strong construct validity and criterion validity (Guscia, Harries, Kirby, Nettelbeck, \& Taplin, 2006). The SNAP does not require specialized training or examiners' qualification except they “... must have good knowledge of the person" (Productivity Commission, 2011, p. 322). Easy tool administration and 20-minute assessment time make it user-friendly. The SNAP manual does not provide specific details on administering and scoring the scale. 
North Carolina Support Needs Assessment Profile (NC-SNAP). The NC-SNAP was developed "to respond to a systemic need identified by the North Carolina Developmental Disabilities Policy Work Group in 1997” (Murdoch Center Foundation, 2013, para. 2). This tool was field-tested in 1997, revised in 1998, and field-tested again in 1999 before its publication. It requires examiners to respond to a 5-point scale questionnaire that includes items related to three support domains (see Table 2). The NC-SNAP was designed to be a simple and easy tool.

However, Hennike et al. (2006) surveyed participants' perspectives on the ICAP, the Developmental Disabilities Profile (DDP), and the NC-SNAP and found that a majority of the participants (53.5\%) ranked the NC-SNAP the least preferred tool. In addition, the NC-SNAP examiners must be certified. It was not recommended to be used by paraprofessionals. To get a certificate, one must register for the training with North Carolina Department of Health and Human Services (NCDHHS; 2013a; 2013b; 2013c) through the Local Management Entity (LME). It is unclear how someone outside of North Carolina would obtain a certificate. Findings and psychometric properties of the NC-SNAP were presented in a way which makes it impossible to compare adult scores with the normative data. There has been limited interest in the NCSNAP outside of the state of North Carolina.

SIS. Developed by Thompson et al. (2004a), the SIS is a standardized support needs measurement that enables comparisons of supports intensity of people with ID/DD. SIS Users Manual explains important information such as how to administer the measurement, psychometric properties of the scale (Thompson et al., 2004b). Details are provided in the following section.

\section{Supports Intensity Scale (SIS)}

\section{SIS Information}

The SIS has three parts: Section 1 Support Needs Scale composes 49 items in six life activities subscales (see Table 2); Section 2 Supplemental Protection \& Advocacy Scale includes 
eight items measuring self-advocacy and self-determination; and Section 3 Extraordinary Medical and Behavioral Support Needs consists of 29 items. A scale administrator interviews two respondents to arrive at ratings of an individual's support needs by frequency, time, and type. Upon completing the assessment, two indices are provided: SIS Support Needs Index (SNI) reflects an individual's overall intensity of support needs compared to the normative group, and SIS Support Needs Profile is a graph displaying an individual's support needs across subscales. An appendix shows a hypothetical report that could be generated from a SIS assessment. Possible scores on each item and subscale are shown in the report, and standard score conversions are also shown. This is being provided to help the reader understand the information the SIS provides and the data that were used in this investigation. Recently, a group of researchers reported that Section 2 Protection \& Advocacy supplemental scale significantly contributed to the SNI scores (Thompson, Shogren, Wehmeyer, Chapman, Tassé, \& McLaughlin, in press). Therefore, the revised SIS will highly likely include scores from the Protection \& Advocacy section to the SNI scores (J. R. Thompson, personal communication, November 1, 2013). The SIS has a number of strengths. First, the SIS Users Manual provides detailed instructions and case studies on how to administer the measurement. When assessors understand how to conduct the SIS, the quality of information derived from the scale is more likely to increase (Thompson, Tassé, \& McLaughlin, 2008). Second, being designed for adults with ID/DD, the SIS was field-tested with a representative group with ID/DD who had different support needs, and norm scores are available. Therefore, standard scores and percentiles derived from the SIS are useful in comparing an individual's scores with the normative data. Third, being used statewide in 18 states (Nygren, 2011) and translated into 13 languages (AAIDD, 2013b), the SIS has been rigorously examined by numerous U.S. and international researchers.

Psychometric indicators of the scale are highly reliable and valid (see Tables 2 and 3). Reliability is the consistency of a measurement. Measurements with strong reliability are 
considered good tools as they provide comparable scores across periods of time and test administrators. According to its manual, the SIS has high internal consistency ${ }^{1}$ and strong testretest reliability ${ }^{2}$, but "Fair" inter-rater reliability ${ }^{3}$ (see Table 2). However, subsequent researchers reported higher inter-interviewer, inter-respondent, and mixed inter-rater coefficients (Thompson et al., 2008). The authors suggested that the reliability coefficients were higher because the participating interviewers had been trained and/or had experience in administering and scoring the SIS.

Validity is an ability of a test to measure construct(s) that it intends to measure. The SIS authors implemented a Q-sort procedure to ensure the scale's content validity. They also reported high content validity, good criterion validity, and strong correlation with the ICAP (see Table 2). Neither age nor gender of individuals affected the SIS subscale scores (Kuppens et al., 2010; Thompson et al., 2004b). The translated versions of the SIS have shown high internal consistency, strong inter-rater reliability, and excellent concurrent and construct validity (Buntinx, 2011).

The support needs information derived from the SIS can be utilized at the individual, organizational, and state levels. At the individual level, personal information (e.g., needs, preferences, goals) and the SIS information provide guidance on appropriate supports that individuals need. Additionally, involving the individuals with ID/DD in assessing their support needs and planning supports can be an empowering experience that leads to better personal outcomes and quality of life (AAIDD, 2013c). At the organizational level, SIS information can be used to classify, plan, and develop a supports system, as well as help service providers make good judgments and informed decisions on individuals' support needs and priorities (Schalock, 2011; Swanton, Walsh, O’Murchu, \& O’Flynn, 2010). Support needs information on

\footnotetext{
${ }^{1}$ Correlations among items of the measurement

${ }^{2}$ Consistency of the measurement across periods of time

${ }^{3}$ Consistency of the measurement across different raters
} 
people can also be used in reporting outcomes, applying for grants, allocating resources, and assessing quality of life (AAIDD, 2013d).

Administrators and policymakers found the SIS useful, particularly in regard to its utility for resource allocation and financial planning. The scale allows policymakers to examine support needs across comparable groups and agencies in a specific area, a state, a multistate region, or the whole country (e.g., Fortune et al., 2009; Kimmich et al., 2009).

\section{Criticisms of the SIS}

Estimating support needs. Arnold et al. (2011) expressed concern about measuring support needs for activities that people do not do in their daily lives. They claim a support needs assessment should measure the support needs in the context of an individual's specific environment, “... not support needed for some standardised set of activities” (p. 260). However, they did not suggest how a standardized support needs assessment would have been developed or how a norm-referenced support needs score could have been generated without a standardized set of activities. Norm-referenced scores are critical to using aggregate data for purposes of resource allocation and public funding where equity is an issue. Without norm-referenced scores, policymakers would not know whether an individual or a group of individuals should receive relatively more or less funding than others.

User-friendliness. In Australia, Guscia et al. (2006) studied the validity of the SNAP by comparing it with the ICAP and the SIS. Participating staff reported rating activities on the SIS caused confusion because the tool required the participants to rate the supports an individual needed to successfully participate in activities that were not currently provided or available in their context. In addition to the fact that the types of community activities included in the SIS were different from ones in Australia and the participants could not envision what those were like, Thompson and Viriyangkura (2013) argued that the "anecdotal report" by Guscia et al. referred to a small sample of workers in institutions which might not be enough to make that 
inference. In fact, others agreed that rating unavailable activities is beneficial for individuals with ID/DD because it prompts everyone involved “... to consider other goals that had not previously been identified within their person centred plan, or even seemed possible" (Swanton et al., 2010, p. 25).

Administering the SIS requires 1-3 hours. Compared to other support needs measurements, the SIS may be considered lengthy and time-consuming. Certainly, if an assessment becomes too onerous to administer, it will not be used no matter how good the information.

Limited population. The SIS was normed on 1,306 adults with ID/DD (Thompson et al., 2004b). Although the sample also had other disabilities (e.g., autism spectrum disorders), norms of other disability groups were not reported as the numbers were too small. Practitioners frequently asked whether the scale could be used with individuals with other disabilities than ID. The AAIDD (2013e) responded that it can, but norms for other disability groups are not available. A number of researchers have implemented the SIS with people with other disabilities (see Table 3). Despite positive findings reported by these researchers, the SIS authors and the AAIDD continue to repeat that no norms for groups other than ID/DD are available.

The SIS was designed for adults with ID/DD, specifically 16 years of age and above. However, since school-aged children are $23.7 \%$ of total American population (US Census Bureau, 2012), a support needs assessment for children is needed. Recently, the Institute of Education Sciences (IES, 2012) awarded a grant of \$1.59 million to the SIS authors to develop and validate the SIS for children (SIS-C) at 5-16 years of age over the period of 2011-2015. The SIS-C is composed of two sections: (a) Support Needs Scale (i.e., Home Living, Community \& Neighborhood, School Participation, School Learning, Health \& Safety, Social Activities, and Advocacy), and (b) Exceptional Medical and Behavioral Needs. Respondents will be asked to rate type of supports that are needed and total daily time needed to provide supports to a child 
with ID/DD (Thompson et al., 2012).

Since its publication in 2004, the SIS has been extensively studied by American and international researchers for various purposes. This SIS literature can be divided into two parts: (1) research published in peer-reviewed journals and (2) research and commentaries reported in materials from a variety of jurisdictions and professional organizations.

\section{SIS Research Reported in Peer-Reviewed Journals}

A search of the professional literature through multiple databases on the Milner Library portal (e.g., ERIC, EBSCO Academic Search Complete, publishers' websites) with keywords as "Supports Intensity Scale" and "support needs" yielded 230 articles on the SIS that have been published in peer-reviewed journals but, after exact duplicates were removed, 85 items remained. Twenty eight articles are poster presentations or plenary summary without details, 14 articles are the assessment review and support needs construct discussion, 20 articles are indirectly relevant (e.g., a survey on assistive technology for people with ID/DD according to SIS subscales), and one article is in French. Table 3 briefly summarizes 22 articles that are directly relevant to the SIS. Statistical analyses used in those studies have included a variety of approaches based on the different purposes of the studies. Four areas that the professional literature provides extensive findings include (a) whether the SIS is measuring a different construct than $\mathrm{AB}$, (b) the reliability of the SIS, (c) the validity of the SIS, and the (d) factor structure of the SIS.

SIS and AB. Whenever a new assessment tool is introduced, one of the most essential questions to investigate is whether it truly measures something different than measurement tools that have come before. Although the constructs of support needs and AB are conceptually different, several researchers have sought to collect data that could indicate whether the SIS is measuring a different construct than what is measured by a variety of $\mathrm{AB}$ scales. 


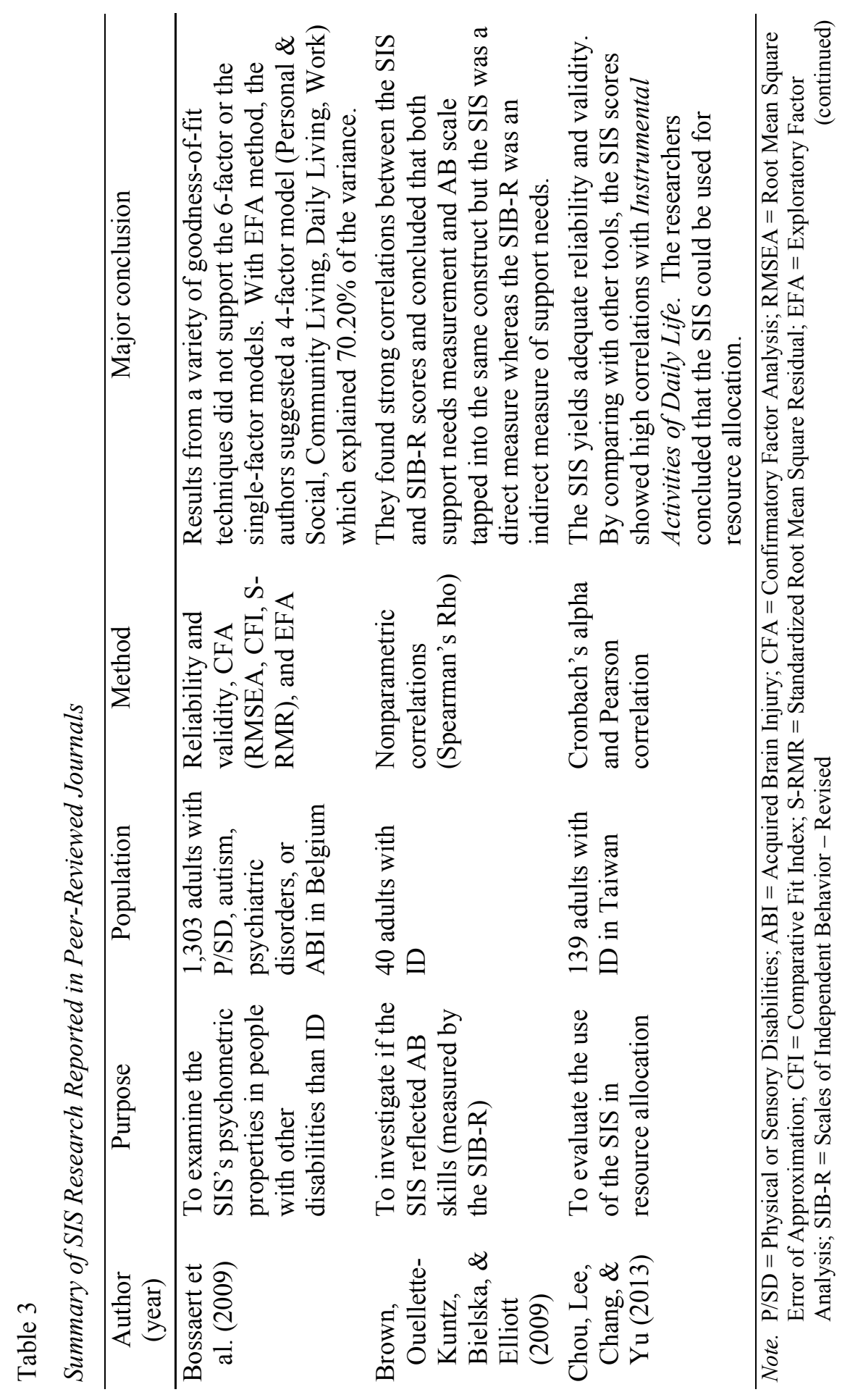




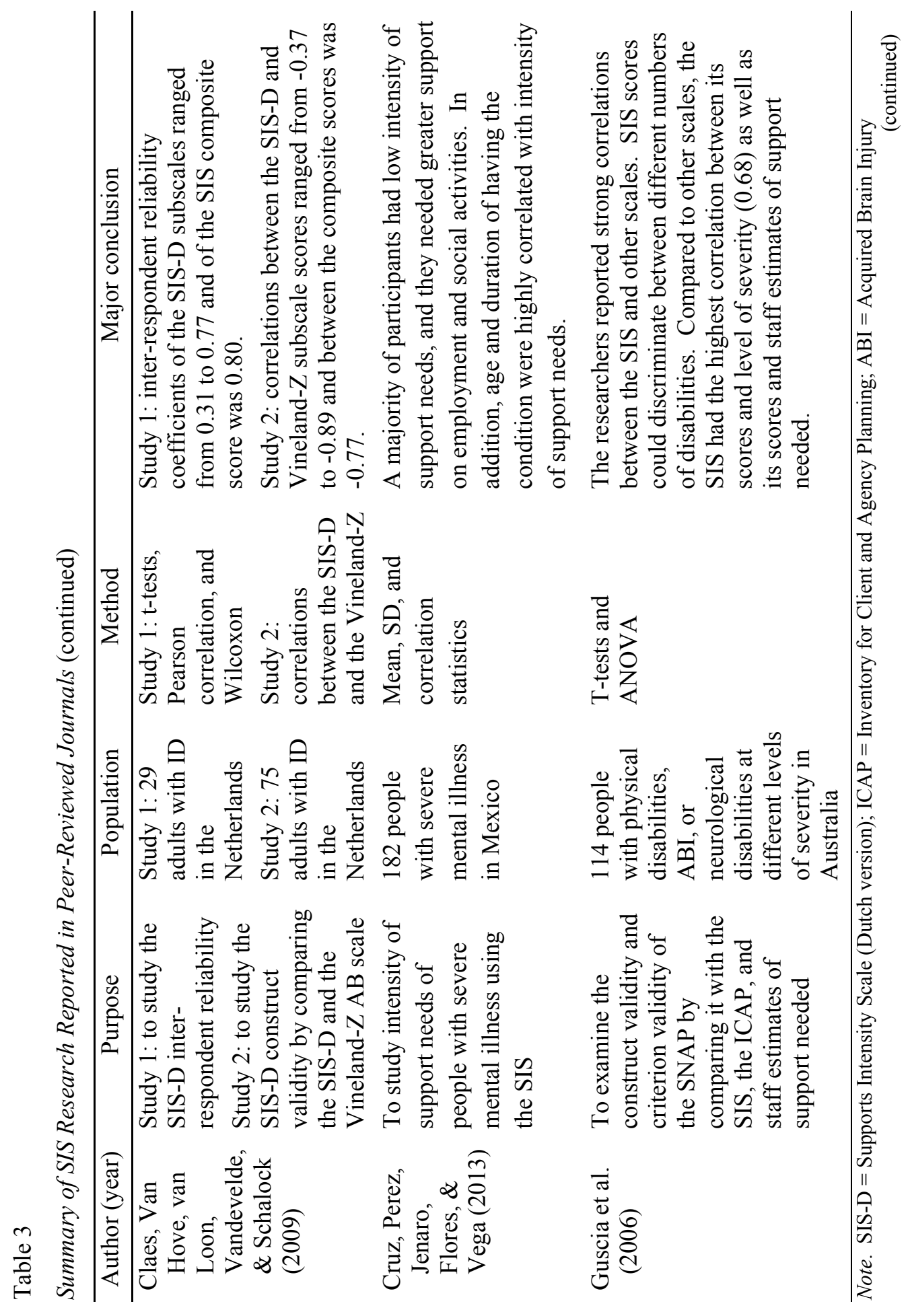




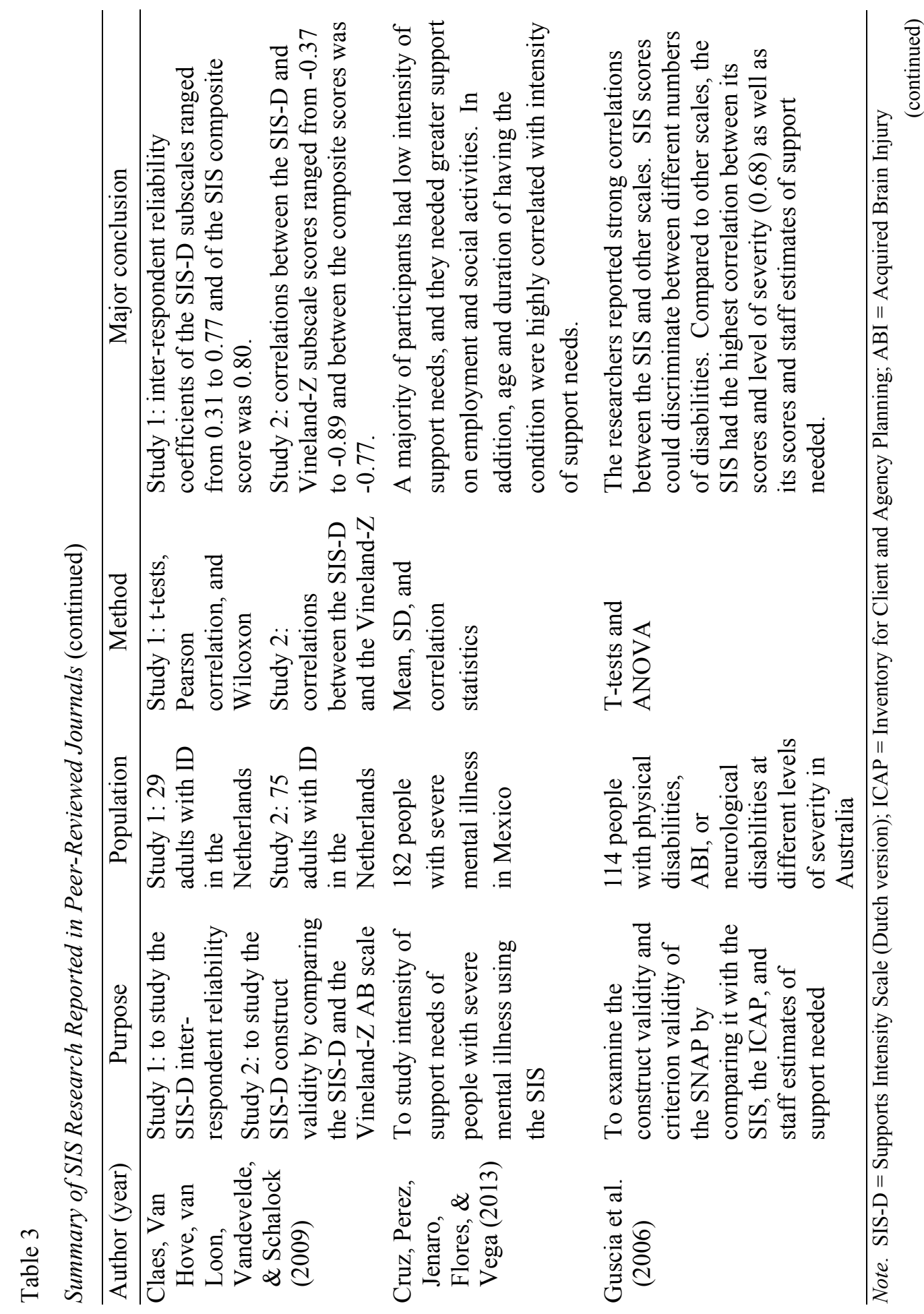




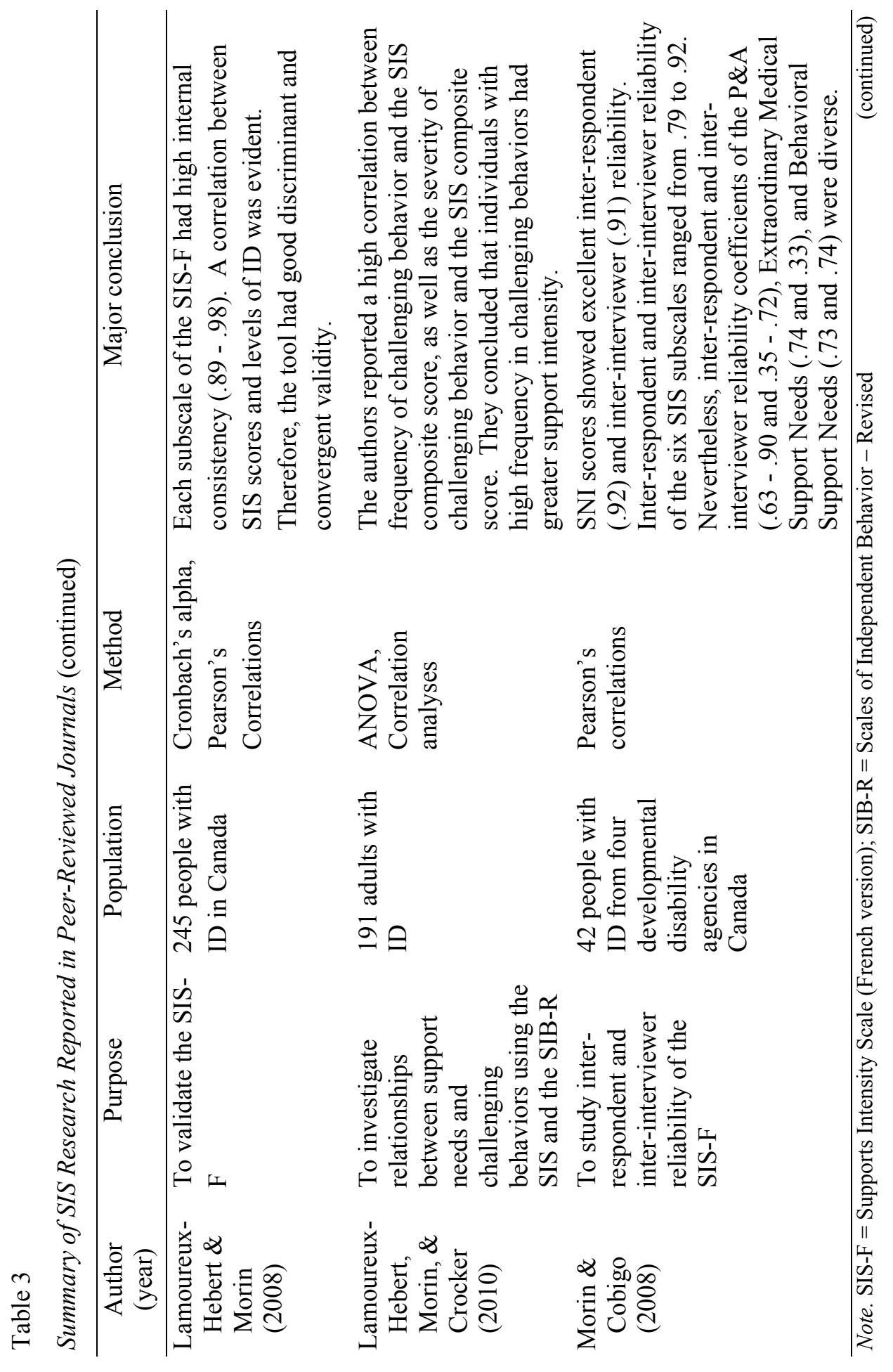




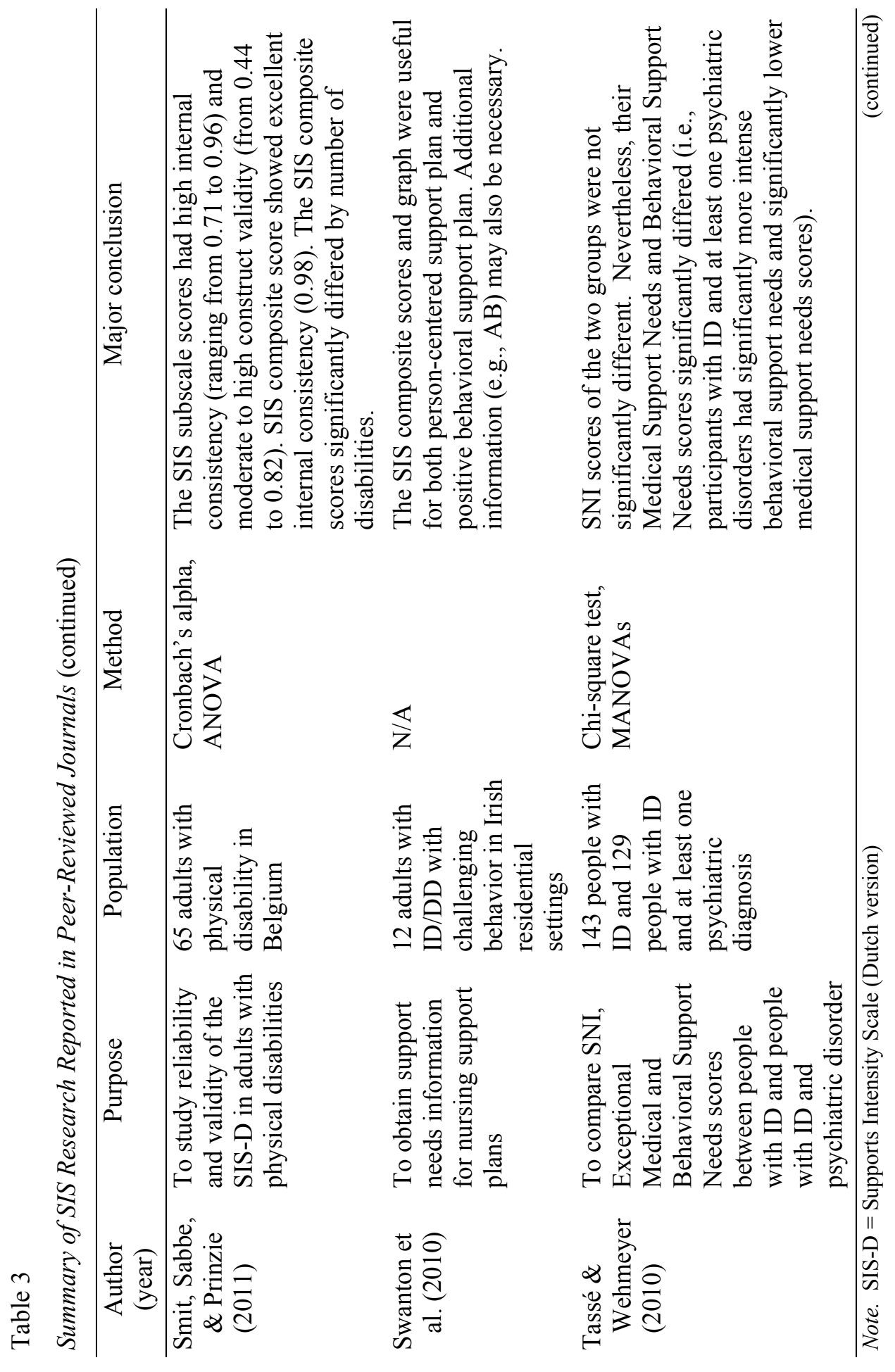




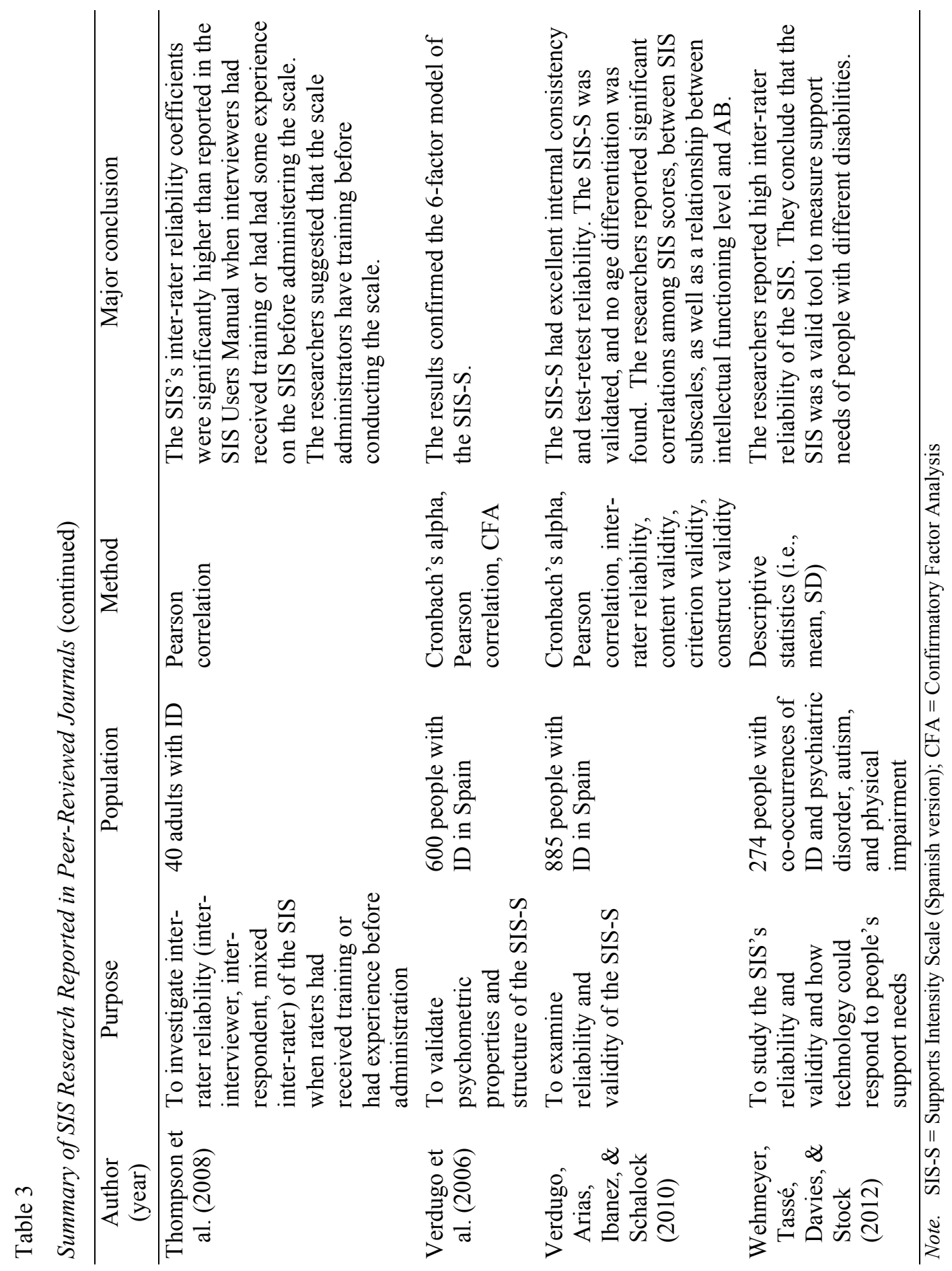


Claes et al. (2009) presented findings on 75 individuals who had been assessed using the Vineland $A B$ scale (VABS) and the SIS (both Dutch translations). Their data confirmed findings reported in the SIS Users Manual (Thompson et al., 2004b) by showing a high negative correlation $(-0.77)$ between the total scores of the two scales, but not such a high correlation that would suggest the scales were measuring the same construct (Thompson et al.'s data indicated coefficients of -0.49 with the VABS and -0.59 with the ICAP, based on sample sizes of 284 and 178 respectively). Claes et al. suggested that the two constructs were logically related, but were different. That is, people with more limited AB skills (and thus relatively lower AB scores) will typically have higher support needs (and therefore relatively higher SIS scores). However, there are other influences on a person's support needs than just AB skills. Guscia et al. (2006) also confirmed prior findings when they examined data from the SIS and the ICAP on 114 people. They reported a high negative correlation (-0.75) and concluded that the SIS (as well as another support needs assessment scale) was measuring a different construct than the AB scale.

However, Brown et al. (2009) and Harries et al. (2005) reached a different conclusion. Brown et al. analyzed data from 40 people who were assessed with the SIS and the Scales of Independent Behavior - Revised (SIB-R), and reported the high negative correlation (-0.89) between SIS and SIB-R scores. They concluded that the two scales were measuring the same construct. They stated that the SIS "is a direct measure of supports needed, while the SIB-R is an indirect measure. A direct measure is more important when the derived information is to be used to plan services for the individual" (p. 954). Harries et al. administered the SIS, ICAP, and Adaptive Behavior Scale - Residential and Community (ABS-RC:2) on 80 people with ID in Australia. They concluded that AB scales and the SIS measured the same construct due to a strong correlation between the scores.

Although there is debate whether problem behavior should be considered within the construct of $\mathrm{AB}$, some $\mathrm{AB}$ scales include a maladaptive behavior section. Lamoureux-Hebert et 
al. (2010) compared the SIS with the SIB-R to investigate relationships between support needs and challenging behaviors of adults with ID as measured in the maladaptive behavior section of the SIB-R. They reported a high correlation between frequency of challenging behavior and the SIS composite score, as well as the severity of challenging behavior and the SIS composite score. They concluded that individuals with high frequency in challenging behaviors had greater support intensity. However, they did not suggest that measures of maladaptive behavior and measures of support needs were assessing the same construct.

SIS reliability. Thompson et al. (2008) noted that indicators of reliability reported in the SIS Users Manual (Thompson et al., 2004b) were strong, with the exception of inter-rater reliability where the coefficients associated with several subscales were in the "fair range" (i.e., from 0.50 to 0.59 ) according to guidelines proposed by Cicchetti and Sparrow (1981). Thompson et al. (2004b) further noted that data on which the SIS Users Manual were based had interviewers who were not trained on how to administer the scale (only provided written instructions), and a very rigorous test of reliability was used because data were collected by different interviewers who interviewed different respondents. Therefore, they set out to assess reliability with interviewers who had completed training on how to conduct assessments to determine if reliability improved when trained interviewers collected data. Also, these researchers investigated inter-interviewer reliability (i.e., pairs of SIS scores generated from different interviewers who interviewed the same respondent), inter-respondent reliability (i.e., pairs of SIS scores generated from the same interviewer who interviewed different respondents on two different occasions), and mixed inter-rater reliability (i.e., pairs of SIS scores generated from the different interviewers who interviewed different respondents) to study variability attributable to having different interviewers and different respondents. Their findings showed markedly higher correlation coefficients for the subscale scores and the total score than were reported in the SIS Users Manual (Thompson et al., 2004b), and based on these findings the SIS was a highly reliable 
scale in terms of inter-rater reliability. They concluded that their findings suggested the importance of training interviewers to assure reliability.

In another study examining inter-respondent reliability, Claes et al. (2009) compared ratings on the SIS when the respondent was the person with the disability who was being assessed to ratings when the respondent was a paid staff member who knew the person with the disability well. They found that ratings from the two groups were quite reliable in the sense that the people whom the staff rated having comparatively higher or lower support needs relative to others in the study were the same people who rated themselves as having higher or lower support needs relative to others. However, the people with ID/DD who were interviewed consistently rated themselves as having less intense support needs than did paid support staff. Although the authors acknowledge there could be several plausible hypotheses for these parallel, but unequivalent, staff and self-advocate ratings, prior research has suggested that many people with ID/DD attempt to cloak their disability because of the stigma associated with being labeled (e.g., see Edgerton, 1993). Also, people with ID/DD may not be fully aware of, and therefore may not fully consider, their vulnerabilities (Greenspan et al., 2001). Conversely, it could be that paid staff have a tendency to overprotect people with ID/DD, and therefore overestimate the support people need. This study highlights the importance of obtaining perspectives from multiple respondents (SIS instructions call for interviewing at least two respondents), and the importance of interviewers probing respondents whenever conflicting information surfaces.

SIS validity. Findings from several studies applicable to judgments about the validity of the SIS have been reported. Several researchers conducted basic psychometric analyses (e.g., internal consistency) relevant to validity on the English and translated versions of the SIS (see Lamoureux-Hebert et al., 2010; Lamoureux-Hebert \& Morin (2008); Morin \& Cobigo (2008); Verdugo et al., 2006; Verdugo et al., 2010). These studies have repeatedly supported the validity of the SIS and aligned with Thompson et al.'s (2004b) findings from the original standardization 
sample. Specific findings from these studies will not be reported in this section. Rather, this section will focus on summarizing findings from studies that attempted to extend evidence for validity beyond the basic psychometric findings reported by Thompson et al.

For example, Weiss et al. (2009) studied the construct validity of the SIS based on the premise that clinical judgments from experts regarding people's support needs should correspond with SIS scores. Five experienced clinicians ranked 50 people with ID/DD as having Low, Medium, or High support needs across a variety of domains. Findings showed experts' ratings were well aligned with SIS subscale and composite scores. These researchers concluded that the SIS was a valid support needs assessment for people with ID/DD.

In a study mentioned earlier, where the efficacy of the SIS was examined in regard to predicting people's need for extraordinary funding in a Midwestern state (where there was a process in place to provide extra funding for people with ID/DD whom had been identified as having extraordinary needs), SIS scores of 117 participants who were receiving extraordinary funding were compared with SIS scores of 157 participants who were not. Additionally, each participant's Developmental Disability Profile (DDP) scores (a combination of multiple measures of personal competence) were included to complete a variety of analyses. Findings revealed that the SIS performed as well or better than the DDP in predicting greater levels of extraordinary funding (Wehmeyer et al., 2009).

Examining the applicability of the SIS to other disability populations can also provide insight regarding the validity of the SIS. If the SIS is a valid support needs measure, it should reflect support needs differences in disability populations other than ID/DD (although norms may be different). Also, Tassé and Wehmeyer (2010) compared SIS scores of people with ID and people with co-occurrences of ID and at least one psychiatric disorder. They reported that, although the SNI scores of both groups were not different, the comorbidity group had significantly more intense behavioral support needs than the group with ID. Differences in 
behavioral scores could reflect unique behavioral support needs of individuals with psychiatric disorders. Furthermore, Wehmeyer, Tassé, Davies, \& Stock (2012) studied the SIS scores of people with co-occurrences of ID and psychiatric disorder, autism, and physical impairment. In addition to high inter-rater reliability of the SIS, this group of researchers concluded that the SIS was a valid tool to measure support needs of different populations.

Smit et al. (2011) investigated the psychometric properties of the SIS (Dutch version; SIS-D) with adults with physical disabilities by comparing SIS scores and Barthel Index (BI; i.e., a measurement of functional abilities in 10 domains of daily life activities) scores. The SIS showed high internal consistency (i.e., 0.98 for the SIS-D composite score and a range of 0.71 for Medical to 0.96 for Lifelong Learning in the SIS-D subscale scores). In regard to construct validity, all of the SIS sections and subscales were significantly correlated (ranged from 0.82 for Lifelong Learning and Health \& Safety to 0.44 for Home Living and Social Activities) with the exception of the Behavior section and the six subscales (ranged from $0.00-0.16$ ). Although the BI did not show a strong relationship with all of the SIS subscales, the authors concluded that the SIS was a good tool for individuals with physical disabilities in terms of intensity of support needed. Cruz, Perez, Jenaro, Flores, and Vega (2013) and Jenaro, Cruz, Perez, Flores, \& Vega (2011) conducted the SIS on the same group of adults with severe mental illness in Mexico, and Guscia et al. (2006) administered the SIS with people with physical disabilities, acquired brain injury, or neurological disabilities in Australia. Those groups of researchers reported that their findings reflected reliability and validity reported in the SIS Users Manual.

Factor analytic studies. The SIS included six subscales, and therefore a 6-factor conceptual model of support needs was implied. Researchers have conducted exploratory and confirmatory factor analysis methods to evaluate a 6-factor multidimensional model and propose alternative models. Below are brief summaries of the factor analytic studies using SIS data that have been published in peer-reviewed, professional literature. 
Harries et al. (2005) investigated the relationship between support needs and AB (conceptual, social, and practical skills) of individuals with ID by conducting an exploratory factor analysis using the SIS (prepublication version in 2002), the ICAP, and the Adaptive Behavior Scale - Residential and Community (ABS-RC:2). They reported that AB scales and the SIS measured a common underlying construct and proposed a unidimensional model. However, results from confirmatory factor analysis supported a single-factor structure for data from the three scales. Verdugo et al. (2006) reported that their factor analytic study confirmed the 6-factor model. Later, Kuppens et al. (2010) conducted confirmatory factor analysis on the 6-factor model and a single-factor model on data from a large sample (i.e., 14,682 adults), and found that their data supported the 6-factor subscale model across gender, age, and disability complexity (i.e., one or multiple disabilities). However, Verdugo et al. (2010) reported findings from a different data set supporting a single-factor structure.

In 2009, Bossaert et al. conducted a confirmatory factor analysis to evaluate the 6-factor model and the single-factor model with adult participants with disabilities other than ID (e.g., acquired brain injury, physical disabilities, sensory impairments, psychiatric or behavioral disorders, autism spectrum disorders) in Belgium. After using multiple goodness-of-fit techniques (i.e., Root Mean Square Error of Approximation or RMSEA, Comparative Fit Index or CFI, and Standardized Root Mean Square Residual or S-RMR) to assess the models' fit, the findings did not support any of the previously suggested models. Therefore, they conducted an exploratory factor analysis to find an alternative solution. Two solutions were suggested: a 4factor model that explained $70.20 \%$ of the variance and a 2 -factor model which accounted for $59.98 \%$ of the variance. The researchers recommended the 4-factor model with Personal \& Social, Community Living, Daily Living, and Work domains using 22 SIS items.

Other SIS research studies. Koritsas et al. (2008) studied the effect of active support (AS) training on perceived support needs using the SIS as the measure of support needs. These 
authors reported significant decreases in Community Living, Lifelong Learning, Employment, Health and Safety, and Social Activities subscale scores after AS training was provided.

However, Home Living remained the same as baseline. Chou et al. (2013) investigated a Chinese version of the SIS and found that, consistent with studies on other translated versions, their data produced strong psychometric indicators mirroring those reported for the English version (Thompson et al., 2004b). They also analyzed SIS data in relation to funding patterns and compared its predictive power with the tools currently used to determine resource allocation in Taiwan. They concluded that SIS scores accounted for all of the information that could be gleaned from other three scales, and therefore was superior to three tools currently used in Taiwan to determine funding for people with ID/DD. In essence, using the SIS could eliminate the time and expense needed to collect data on three separate assessments, and the results of the assessment would also be useful for support needs planning.

\section{SIS Research and Reports in Grey Literature}

Several findings from data that were collected and analyzed on the SIS and have been reported in sources other than professional journals over the past decade. Debachere (1995) pointed out that people "generally prefer to describe grey literature rather than to define it" (p. 94). For the purpose of this review, the grey literature on the SIS includes anything other than publications in peer-reviewed, professional journals. There are four major sources of grey literature where SIS research findings were reported: (a) the SIS Users Manual where psychometric findings from the English version standardization sample are reported, (b) a series of four AAIDD White Papers that were published in 2008 that include numerous short articles reporting findings from data collected on both the English and translated versions of the SIS, as well as ways in which the SIS is used at the individual, organizational, and jurisdictional levels, (c) reports published by the Human Services Research Institute (HSRI), and (d) a cornucopia of reports that have been published by other organizations or governmental agencies. 
SIS Users Manual. The SIS Users Manual (Thompson, 2004b) is a 128-page book that was published by the AAIDD to coincide with the release of the scale. The first chapter provides the context and rationale for creating the SIS through reviewing societal changes in the way that people with ID/DD are understood, and by providing a short literature review related to support needs and community living. Chapters 2 and 3 provide detailed instructions on the administration and scoring of the SIS, and Chapter 4 provides four case studies illustrating how the SIS can be used to develop individualized support plans. A variety of ways in which aggregate SIS scores can be used at the organizational and jurisdictional level are presented in Chapter 5. And finally, Chapter 6 presents technical information pertinent to the psychometric evidence underlying the scale.

The SIS (Thompson et al., 2004a) and SIS Users Manual (Thompson et al., 2004b) were reviewed by two experts in the area of psychological testing and measurement for publication in the Mental Measurements Yearbook (MMY). Although the MMY was traditionally a print publication marketed to academic institutions for their library reference sections, today's MMY is a searchable, web-based database to which academic institutions can subscribe. Reviews from Loew (2005) and Pittenger (2005) were published shortly after the SIS came on the market and continue to be included in the MMY to this day. Both reviewers were quite positive about the new tool, noting that the SIS filled a unique niche by measuring an important construct for which other assessments only provided indirect measures. Additionally, both reviewers had high praise for the Users Manual indicating that administration instructions were clear and the examples were helpful. Finally, both were impressed with the psychometric findings and concluded the SIS was a sufficiently reliable and valid tool. Loew, however, cautioned that due to the mixed inter-rater reliability findings potential users should pay close attention to training those who complete the assessments.

The SIS Users Manual was also reviewed by Davison (2005) for the Journal of 
Intellectual Disability Research shortly after the SIS came into print. Although she provided incorrect information in several places (e.g., she indicated a 4-point scale was used to rate each dimension when actually a 5-point scale is used; she reported that there were only 28 items), her overall conclusion was positive, stating that "it is likely to provide useful additional to many services (sic) basic assessment and planning tools."

AAIDD White Papers. Schalock, Thompson, and Tassé (2008a; 2008b; 2008c; 2008d) served as editors for four booklets (i.e., the White Paper series) published by the AAIDD to provide examples from around the world of how the SIS was being used by researchers, policymakers, and practitioners. Each booklet contained several short articles. The editors reported that article authors were selected because they had initiated successful and innovative SIS research and/or demonstration projects. The four booklets were (a) Psychometric Properties of the SIS, (b) International Implication of the SIS, (c) Resource Allocation and the SIS, and (d) Relating SIS Information to Individual Service Plans.

Many of the articles focusing on the psychometric properties of the SIS were preliminary findings from studies that were later published in peer-reviewed journals. These studies were reviewed in the prior section and will not be reviewed here. However, it is particularly noteworthy that psychometric findings from three translated versions of the SIS (the French, Catalan, and Dutch translations) virtually mirrored the psychometric findings from the English version reported by Thompson et al. (2004b) in the SIS Users Manual (see Buntinx, 2008; Giné, 2008; Lamoureux-Hebert \& Morin, 2008; Morin \& Cobigo, 2008). Additionally, researchers from Israel, Italy, Spain, and Taiwan presented information regarding their translation process, initial efforts at data collection, and experiences using the scale. The common theme of these articles was the SIS had strong face validity in regard to its application with diverse populations in the different countries.

The Resource Allocation and the SIS booklet included a contribution from Smith and 
Fortune (2008) of the HSRI. At the time their article was published, HSRI was working with several states on resource allocation for ID/DD populations (and this work continues to the current day). Other articles in this booklet were from state government officials in Washington (Weber \& Stern, 2008), Louisiana (LeVelle, \& Meche, 2008), and Nebraska (Severance \& Campbell, 2008) with responsibilities for resource allocation, as well as an article from an administrator who was employed by a large service provider in the Netherlands (van Loon, 2008). The central theme of these articles was that ID/DD funding could be distributed more efficiently and equitably if SIS scores (i.e., a measure of support needs) were considered in addition to (or in lieu of) traditional system-based factors driving funding formulas (e.g., group home residents receive greater allocations than individuals who lived in their family homes).

Another major theme of the resource allocation articles was that SIS data must be accompanied by other data in order for a funding formula to be worthwhile. In fact, all article authors either explicitly stated or implied that funding formulas should not be based on a single assessment, and this is as applicable to the SIS as any other assessment. For example, the State of Washington developed their funding formula using SIS data and "locally added items" such as questions about sleep patterns, seizures, home treatments or therapies, amount of protective supervision needed, diabetes management, and other challenging behaviors (Weber \& Stern, 2008). In Louisiana, SIS scores and personal information (e.g., diagnosis, age, geographic location) were used in a regression analysis to identify predictors of service costs. Later called LA Plus, this resource allocation approach included questions about material support needs (e.g., power wheelchair, augmentative communication devices), vision and hearing needs, communication assistance needs, protective supervision needs, behavior needs, medical services needs, risk needs, sleep needs, and pain relief needs, as well as medical diagnoses, current medications, a cursory vocational assessment, a brief satisfaction questionnaire, and current and preferred supports and living arrangements questions (LeVelle, \& Meche, 2008). There was also 
a report of a service provider organization (Arduin) in the Netherlands that had recently undergone a dramatic transformation. Eight years earlier, this organization was totally "facilitybased" (i.e., operating all services from an institution), but had undergone a dramatic conversion to become totally community based (all residents living in neighborhood homes and working in community settings). The SIS, along with a Quality of Life scale and several other measures, were used by Arduin to develop a resource allocation formula that provided a basis for creating individual budgets. Outcome measures verified that Arduin's new approach to funding people instead of programs resulted in more efficient and effective use of financial resources (van Loon, 2008).

HSRI reports. The HSRI has provided technical assistance on Home and CommunityBased Services quality enhancement techniques and resource allocation models to states on behalf of the Centers of Medicare and Medicaid Services (CMS) for a number of years (J. Fortune, personal communication, July 26, 2013). They issued a number of reports on resource allocation and funding formulas for adult ID/DD populations (for a partial listing, see http://www.hsri.org/topics/resource-allocation-and-planning-models/). Although several reports make reference to the SIS and how it has been used in different states, reviewing state-by-state information is beyond the scope of this review. However, there are two reports that have the SIS as the central topic. Their most recent report was a literature review of peer-reviewed and grey literature on the SIS related to the internal consistency, inter-rater reliability, test-retest reliability, face validity, content validity, criterion validity, and construct validity (Fortune, Agosta, \& Bershadsky, 2011). Consistent with the more expansive review of literature presented in this chapter, HSRI's literature review exhibited that the body of research on the psychometric properties of the SIS show that it is a valid and reliable scale.

In Developing Individual Budgets and Reimbursement Levels Using the Supports Intensity Scale (Kimmich et al., 2009), a juried document funded by CMS, the HSRI noted "the 
SIS has gradually become the most frequently-chosen assessment tool for developmental disability service systems in the United States, gradually replacing the ICAP” (p. 20). The authors reviewed processes that have been used in states to arrive at funding formulas, detailing information from Georgia, Colorado, Utah, Louisiana, and Washington. This report essentially offers a how-to guide for a jurisdiction that wants to use the SIS and move forward in assuring that public funding is distributed efficiently and equitably.

Other grey literature. An Internet search yielded numerous web sites and PDF files that provide information on the SIS with varying degrees of quality and complexity. Much of the information is focused on how the SIS is used by a jurisdiction, such as is provided by government agencies focused on developmental disabilities (DD) in North Carolina (NCDHHS, 2013d), Colorado (Colorado Department of Human Services or CDHS, 2013), New Hampshire (Community Support Network, 2013), Pennsylvania (Pennsylvania Department of Public Welfare or DPW, 2013), and Washington (Washington State Department of Social and Health Services or DSHS, 2013).

Other information found on the Internet focuses on using statistical models to evaluate the extent to which funding formulas are aligned with a variety of policy goals and how the SIS may help improve the alignment. The National Disability Authority (NDA, 2011) in Ireland provided the most impressive report of this type. Also, there are many Internet sources which offer praise or concerns about how the scale is being used, sometimes offering no data to support conclusions and other times offering only selective data or data that would appear to be hard to confirm. For example, the pro-institutionalization organization called the Voice of the Retarded (VOR, 2013) expressed concern that the SIS identified 20\% of people living in ICF/MR facilities in Louisiana for movement to less restrictive residential placements. However, no data were provided in regard to why these people would be worse off living in homes in community settings. 


\section{Factor Analysis and Cluster Analysis in Special Education}

\section{Overview of Factor Analysis}

Factor analysis has been a valuable statistical tool among researchers in social sciences for many years. It enables investigations of components of psychological constructs that cannot be directly observed or measured (known as latent factors or common factors). Identifying latent factors is accomplished by examining relationships among variables in a large data set.

Specifically, factor analysis provides a means to "uncover a (usually) smaller number of latent variables by studying the covariation among a set of observed variables" (Long, 1983, p.11). Because latent factors account for the variability among multiple observed variables, factor analysis also enables researchers to explore a data set for possible variable reduction (Kim \& Mueller, 1978). For example, if data show that variation in 15 survey items related to personal satisfaction variables can be explained by a single latent factor, in future investigations it may be appropriate to collect data on just a few variables (e.g., satisfaction with living conditions, satisfaction with work, satisfaction with relationships) with the highest factor loadings to measure the latent factor (i.e., personal satisfaction).

Factor analysis is typically divided into two broad categories of approaches, exploratory and confirmatory factor analysis. Exploratory factor analysis (EFA) aims to identify the underlying relationships between measured variables when researchers do not have a prior hypothesis about factors or patterns of measured variables. When researchers do not specify the structure of the relationships among the variables in a factor model, all model parameters are free to vary. It is important for researchers to understand that the strength of EFA is also its weakness. Because EFA does not place any constraints on data, the strength of the approach is that an innovative factor structure may emerge that researchers may not have thought of otherwise. However, there is also a chance (due to lack of constraints) that a misleading factor structure may emerge that is merely a fluke of random data. Also, EFA can be considered to be an arbitrary 
approach to obtaining an understanding of a construct if researchers approach a data set atheoretically. During the analysis process, researchers must make a number of decisions before arriving at a solution. If there is no theoretical lens through which to examine data, there is a risk that a misguided factor structure may emerge (Long, 1983; Norris \& Lecavalier, 2010).

The limitations of EFA can be addressed by using confirmatory factor analysis (CFA) techniques. The purpose of CFA is to examine whether data confirm a hypothesized or predicted model. Unlike EFA, CFA imposes constraints on a factor structure, and the constraints determine relationships among different variables and different factors. In essence, CFA shows how well data support (or do not support) a specific factor structure (i.e., CFA indicates the relative robustness of a model). EFA and CFA are often used in a complimentary fashion. EFA is used to generate a plausible model using one data set, and CFA is used to test the model with another data set. Of course, the two data sets must be equivalent in terms of having the same types of participants and using the same types of measures (Long, 1983; Norris \& Lecavalier, 2010).

Finally, it is important for researchers to realize that the distinction between using EFA to explore a factor structure and CFA to confirm a factor structure is blurred. Long (1983) pointed out that "the confirmatory factor model can be used in an exploratory fashion" (p. 15). For example, researchers may find that there are several equally reasonable models to investigate based on prior research and theory. In such an instance, CFA could be applied with minimal restraints placed on the relationships between observed variables and/or latent factors. The purpose of such a study would be to explore whether data fit one model better than another (as opposed to a rigid test designed to confirm a specific factor structure). Researchers using CFA must determine what constraints to place on a model. The extent of constraints is a good indication of whether CFA is being used for purposes that are more exploratory or confirmatory in nature.

A final caution with factor analysis, whether EFA or CFA, concerns the quality of data 
entered into an analysis. Although corrupt data will ruin any scientific investigation, due to the large data sets used in studies featuring factor analysis and because statistical computing programs function to apply algorithms that will impose a structure (or evaluate a structure) on a data set, corrupt data can yield a bogus factor structure. The "garbage in/garbage out" phenomena will come into play if data are of poor quality because measures were not collected with fidelity or because measures are poor proxies for whatever construct is being investigated. That is, a structural model may emerge that accurately represents data from a purely statistical standpoint. However, such a model lacks validity (i.e., garbage out) because it was established on invalid data (i.e., garbage in).

\section{Applications of Factor Analysis to ID/DD}

For over a century, factor analytic techniques have been used for further understanding of psychological constructs that are critical to the field of ID/DD. In particular, there is a body of literature on the factor structure of the constructs of intelligence, $\mathrm{AB}$, and support needs. The literature is quite expansive in relation to intelligence, relatively mature in terms of $A B$, and it is just emerging in regard to support needs. Conclusions regarding the factor structure of each construct are provided in this section.

Intelligence. Perhaps the best well-known factor analytic study in the history of psychology was the very first one. Namely, Charles Spearman's 2-factor theory of intelligence posited that a single general ability factor ( $g$ factor) was the key determinant in human intelligence. Spearman developed the earliest factor analytic techniques, and he collected data from intelligence tests to support the notion that human intelligence was general and global because correlations between specific abilities (s factors) were quite high (Kail \& Pellegrino, 1985; McGrew \& Flanagan, 1998).

Louis Thurstone challenged Spearman's conclusions about intelligence, and he made considerable contributions in furthering the sophistication of factor analytic techniques in the 
process. Thurstone concluded that Spearman's findings and conclusions were an artifact of using an overly narrow range of mental tests. When using a more diverse range of mental tests, Thurstone's data supported the first widely accepted theory of multiple intelligences (i.e., seven primary mental abilities; Kail \& Pellegrino, 1985; McGrew \& Flanagan, 1998).

Since the 1940s, increasingly sophisticated hierarchical intelligence theories have emerged in the attempt to reconcile Spearman's and Thurstone's positions. Correspondingly sophisticated factor analytic processes were essential to developing these theories (Kail \& Pellegrino, 1985; McGrew \& Flanagan, 1998). Hierarchical models of intelligence are based on the premise that there are large (more general) factors of intelligence that underlie more specific intellectual abilities. However, somewhat akin to a spider's web, the narrower specific factors and general factors are intertwined and interact with one another (Hunt, 2011; Thorndike \& Thorndike-Christ, 2010).

Although hierarchical models have been considered to be the most valid structural models of intelligence by today's leading intelligence theorists (Hunt, 2011; Thorndike \& Thorndike-Christ, 2010), there are some who remain convinced that intelligence is better understood in non-hierarchical way. Guilford's Structure of the Intellect model posits over 100 distinct intellectual abilities. It is often pictured as a Rubik's Cube ${ }^{\circledR}$, with many different combinations of intelligence interacting with one another to present a wide variety of mental processes (Kail \& Pellegrino, 1985). Howard Gardner's Theory of multiple intelligences (MI theory) posits nine separate intelligences. It may be the best-known intelligence theory among educators (Klein, 1997).

However, non-hierarchical models of human intelligence lack empirical support. Carroll's (1993) review of data from factor analytic research in the area of intelligence showed very little support for either Guilford's or Gardner's models. Gardner's theory, in particular, has come under severe criticism by cognitive psychologists due to the fact that so many educators 
have suggested MI theory has important instructional implications for classroom teachers (Klien, 1997; Waterhouse, 2006). Although the critiques are many and the debate is complex, Klien (1997) summed up the harshness of the critiques of Gardner's theory very succinctly by asserting "MI theory offers a level of analysis neither empirically plausible nor pedagogically useful" (p. 389).

AB. The factor structure of AB certainly has not attracted the same level of attention or controversy as human intelligence. For one thing, only people in the field of ID/DD tend to be interested in $\mathrm{AB}$ whereas interest in human intelligence spans multiple fields and multiple disciplines. Also, $\mathrm{AB}$ is a construct centered on achievement (i.e., what a person does or does not do). In contrast, human intelligence is associated with aptitude, and it is considered to be a desirable human trait.

Nevertheless, the explosion of $\mathrm{AB}$ scales that came on the market during the 1960s until 1980s paved the way for a significant body of literature on the factor structure of the construct. Thompson, McGrew, and Bruininks (1999) reviewed 31 studies that were published in peerreviewed professional journals from 1968 to 1995 . They concluded that AB (as measured in existing scales) consists of five domains (i.e., personal independence, responsibility, cognitive/academic, physical/developmental, and vocational/community). The authors also concluded that no single $\mathrm{AB}$ scale completely measured all five dimensions.

These conclusions would appear to be consistent with studies published since Thompson, McGrew, and Bruininks' (1999) review. For example, Wei, Oakland, and Algina (2008) and Aricak and Oakland (2010) explored the factor structure of the Adaptive Behavior Assessment System-II (ABAS-II), whereas Arias, Verdugo, Navas, and Gomez (2013) investigated the factor structure of the Diagnostic Adaptive Behavior Scale (DABS). Although each research team reported a factor structure consistent with some of the domains identified by Thompson et al., none of them reported a factor structure that was consistent with all five of the dimensions that 
were identified in Thompson et al.'s literature review.

Support needs. Earlier in this chapter, it was reported that four support needs measurements of people with ID/DD (i.e., the I-CAN, the SNAP, the NC-SNAP, and the SIS) have been developed. To date, the SIS is the only scale where data have been collected for the purpose of completing an EFA and/or CFA. So far, four studies have been published. Harries et al. (2005) investigated the relationship between support needs (measured by the SIS prepublication version in 2002) and AB (conceptual, social, and practical skills) of individuals with ID by conducting an exploratory factor analysis. They reported that both Support Needs scale and $\mathrm{AB}$ scales measured a common underlying construct and proposed a unidimensional model.

Verdugo et al. (2006) and Kuppens et al. (2010) reported that their separate factor analytic studies supported the 6-factor model (implied from Thompson et al., 2004a) whereas Bossaert et al.'s (2009) confirmatory factor analysis conducted on adult participants with disabilities other than ID supported neither the 6-factor model nor the single-factor model. After implementing exploratory factor analysis, Bossaert et al. suggested a 4-factor model (i.e., Personal \& Social, Community Living, Daily Living, and Work) with 22 SIS items which explained $70.20 \%$ of the variance.

\section{Overview of Cluster Analysis}

Whereas factor analysis uses item variables as the unit of analysis and collapses multiple observed variables into categories (i.e., latent factors), cluster analysis uses individuals as the unit of analysis. The purpose of conducting cluster analysis is to determine if people can be classified or placed into subgroups based on shared characteristics. Cluster analysis is a family of statistical techniques used to classify objects or people into homogeneous subgroups. The main objective of cluster analysis is to maximize both within-group homogeneity and between-group heterogeneity (Lattin, Carroll, \& Green, 2003). 
Cluster analysis was first used in the field of biology. However, it has been implemented in diverse fields over the past twenty years (Aldenderfer \& Blashfield, 1984; Lattin et al., 2003). Cluster analysis can be performed in two ways: Hierarchical Clustering and Partitioning. The hierarchical clustering techniques consider that nearby objects (e.g., participants in this dissertation) are more related than further away objects. Therefore, it connects objects in proximity and with similarity, one by one, to form homogeneous clusters. In contrast, partitioning techniques divide a group of objects into a predetermined number $(k)$ of groups where within-group similarity and between-group difference are optimal. The $k$ number can be derived from statistical procedure or an analyst's expertise. After choosing the best clustering model, researchers name each cluster by the groups' characteristics (Lattin et al., 2003).

\section{Applications of Cluster Analysis to ID/DD and Special Education}

There were researchers who tried to group individuals with ID/DD using cluster analysis in the past decade. For example, Zheng et al. (2012) classified individuals with ID/DD by sociodemographics, and Boström, Broberg, and Bodin (2011) grouped people with ID/DD according to temperament profile. Also, Soenen, Berckelaer-Onnes, and Scholte (2009) analyzed data related to IQ, $\mathrm{AB}$, and behavioral functioning of 73 children and adults with Mild ID to identify possible subcategories of this diverse (and often overlooked) group. However, none of researchers has clustered people with ID/DD by support needs.

In addition to the area of ID/DD, cluster analysis has been used to subdivide a variety of different disability groups. For example, Bitsika, Sharpley, and Orapeleng, (2008) were interested in categorizing people with autism, Al-Yagon, (2012) and Nunez et al. (2011) investigated subgroups of children with LD, and Miller (2010) explored potential subgroups of people who were deaf and hard of hearing. Overall, researchers in the field of special education have used cluster analysis to divide children (e.g., Almqvist, 2006), adolescents (e.g., Farmer et al., 2011), and adults (e.g., DeJong \& Donders, 2010) with disabilities into homogeneous groups by 
academic ability, psychological characteristics, and physical traits. Cluster analysis techniques were also used to classify general and special educators (e.g., Prucher \& Langfeldt, 2002), as well as caregivers and families (e.g., Brown \& Rodger, 2009; Morales, Lopez, \& Mullet, 2011).

\section{Chapter Summary}

Historically, ID has been conceptualized as a defect within a person, and people with ID/DD have been understood and classified based on the extent of their deficits and limitations. However, over the past several decades a social-ecological conceptualization of ID has gained acceptance and efforts have been made to understand people with ID/DD by their needs for extra supports (that most others in the population do not need). The introduction of support needs assessments has been an important advancement in the quest to better understand people with ID/DD by their support needs. The most prominent of all support needs assessments is the SIS. Peer-reviewed and grey literature show that the SIS is a reliable and valid measurement tool based on a wide range of psychometric indices.

However, there are gaps in the literature that need to be addressed to further knowledge about the SIS, supports needs assessments, and the nature of support needs of people with ID/DD. In particular, research on factor structure of the SIS is inconclusive. Additional factor analytic studies are needed to evaluate the various factor models that have been suggested by prior researchers. Also, no prior researcher has employed cluster analysis techniques to analyze SIS data. Cluster analysis is needed to indicate the extent of empirical support for classification approaches that consider information on support needs and other important life conditions. The next chapter presents a series of analyses which use (a) factor analysis to shed new light on the factor structure of the SIS as well as domains of the support needs of people with ID/DD and (b) cluster analysis to identify categories of people with ID/DD by support need characteristics. 


\section{CHAPTER III}

\section{RESEARCH DESIGN}

\section{Statement of the Problem}

Considerable progress has been made in understanding people by support needs and in planning supports. Nevertheless, there is still much to be learned in regard to understanding people by their support needs as well as the nature of supports that truly lead to higher quality lives and improved personal outcomes. This study extended the knowledge base through a systematic analysis of a statewide data set of adults with intellectual and related developmental disabilities (ID/DD). Cluster analysis procedures were applied to identify subgroups within the ID/DD population that shared similar support needs characteristics. Additionally, confirmatory factor analysis (CFA) procedures were applied to investigate the empirical support for alternative latent factor models of support needs of people with ID/DD.

\section{Research Questions}

This study contributed to a research-based understanding of support needs of people with ID/DD by (a) investigating classification structures according to commonalities in support needs, personal circumstances, and life conditions, and (b) investigating factor structures of the support needs construct based on support needs assessment and personal competence data. The following research questions have guided data analysis procedures:

1. What multiple grouping model, based on differing support needs, is generated from a statewide sample of people with ID/DD using cluster analysis? 
2. Based on CFA, which of the following structural model of support needs proposed in prior professional literature is best supported by data from a statewide sample of people with $\mathrm{ID} / \mathrm{DD} ?$

a. A 7-factor model aligned with the subscale structure of the SIS (i.e., the six subscales contributing the SNI score and the Supplemental Protection \& Advocacy [P\&A] Scale). The basis for this model is from research completed by Verdugo et al. (2006) and Kuppens et al. (2010), as these two research teams reported that data from the six SIS subscales supported a 6factor model, and it would therefore be logical to assume that if data from a seventh subscale (i.e., the P\&A scale) were added, a 7-factor model would emerge (Model 1 in Appendix A).

b. A 7-factor hierarchical model, where a higher-order (i.e., second-order) factor accounts for shared variance between the seven first-order factors (i.e., data aligned with the six subscales contributing the SNI score and the P\&A scale), but the seven first-order factors are distinct from one another. This model is the factor structure implied by the revised SIS (i.e., where the P\&A scale will be included as a subscale and contribute to the SNI score which is also known as composite score). An SNI score (i.e., composite score) representing overall intensity of support needs suggests a higher-order global support needs factor (Model 2 in Appendix A).

c. A 5-factor model, based on Bossaert et al.'s (2009) 4-factor structure that included 22 SIS items, plus the addition of a new factor associated with the eight items on the P\&A scale (Model 3 in Appendix A).

d. A 5-factor model, based on Bossaert et al.'s (2009) 4-factor structure with a new factor associated with the eight items on the P\&A scale. The distinction between this model and the model described above under "c" is that all variables from the SIS subscales were considered, not just the 22 items Bossaert et al. indicated were relevant (Model 4 in Appendix A).

e. A single factor structure. Harries et al. (2005) reported that their support needs and personal competence data were best explained by a single factor which they felt was 
reflective of a person's global support needs. If support needs are a unidimensional factor (i.e., does not have multiple dimension), the introduction of additional measures of support needs (i.e., the eight items on the P\&A scale) should not affect the factor structure (Model 5 in Appendix A).

\section{Research Design Procedures}

\section{Collection of Data}

Data used in this dissertation are secondary data derived from the Comprehensive Home and Community-Based Services Waiver (CW) Program, a state-level waiver program for individuals with ID/DD in Colorado. In 2005, the State of Colorado was required by the Centers for Medicare and Medicaid Services (CMS) to change its reimbursement procedure from "Community Centered Boards' decisions" to "a uniform, consistently applied rate setting methodology" (Navigant, 2007, p. 4). Therefore, Colorado's human services authority hired the Human Services Research Institute (HSRI) to develop appropriate reimbursement models for their adult service programs in the DD system. The CW Program specifically provided services for individuals with ID/DD who lived in residential settings (outside their family homes). Those services included residential habilitation, day habilitation, supported employment, and other services for those who needed $24 \times 7$ assistance.

Although various types of information were gathered from a number of sources (e.g., staff wages, staffing ratio, individuals' personal data), the State of Colorado used the SIS as the major tool to measure support needs of their $4,003 \mathrm{CW}$ consumers. Demographic data of the participants are presented in Table 4. The state trained case managers to administer the SIS, entered data into an SPSS file, and delivered the file to the HSRI. The HSRI used over 650 variables in their analysis. The funding models proposed to Colorado can be found in Navigant's (2007) full report. 
Table 4

CW Participants' Demographic Data

\begin{tabular}{|c|c|c|c|}
\hline & Characteristics & $\#$ of CW participants & $\%$ of CW participants \\
\hline \multirow[t]{2}{*}{ Gender } & Male & 2,352 & 58.8 \\
\hline & Female & 1,651 & 41.2 \\
\hline \multirow[t]{9}{*}{ Age } & Below 16 years & 2 & 0.0 \\
\hline & $16-19$ years & 22 & 0.5 \\
\hline & $20-29$ years & 715 & 17.9 \\
\hline & $30-39$ years & 810 & 20.2 \\
\hline & $40-49$ years & 1,131 & 28.3 \\
\hline & $50-59$ years & 804 & 20.1 \\
\hline & $60-69$ years & 341 & 8.5 \\
\hline & 70 years and above & 128 & 3.2 \\
\hline & Unknown & 49 & 1.2 \\
\hline \multirow[t]{3}{*}{$\begin{array}{l}\text { Community } \\
\text { safety risk }\end{array}$} & $\begin{array}{l}\text { Community safety } \\
\text { convicted }\end{array}$ & 79 & 2.0 \\
\hline & Need supervision & 193 & 4.8 \\
\hline & No observed problem & 3,731 & 93.2 \\
\hline \multirow[t]{8}{*}{ Residence } & 4-Bed group home & 105 & 2.6 \\
\hline & 5-Bed group home & 212 & 5.3 \\
\hline & 6-Bed group home & 246 & 6.1 \\
\hline & 7-Bed group home & 150 & 3.7 \\
\hline & 8-Bed group home & 404 & 10.1 \\
\hline & Host home & 1,975 & 49.3 \\
\hline & Other IRSS setting & 905 & 22.6 \\
\hline & Unknown & 6 & 0.1 \\
\hline \multirow{11}{*}{$\begin{array}{l}\text { SIS } \\
\text { percentile }\end{array}$} & $1-10$ & 212 & 5.3 \\
\hline & $11-20$ & 305 & 7.6 \\
\hline & $21-30$ & 370 & 9.2 \\
\hline & $31-40$ & 517 & 12.9 \\
\hline & $41-50$ & 411 & 10.3 \\
\hline & $51-60$ & 495 & 12.4 \\
\hline & $61-70$ & 650 & 16.2 \\
\hline & $71-80$ & 493 & 12.3 \\
\hline & $81-90$ & 477 & 11.9 \\
\hline & $91-100$ & 59 & 1.5 \\
\hline & Unknown & 5 & 0.1 \\
\hline
\end{tabular}

Note. IRSS $=$ Individual Residential Services and Supports 


\section{Measurement}

As mentioned earlier, the SIS was published in 2004 and has been used extensively in the U.S. and around the world. The SIS includes three sections. Section 1 Support Needs Scale gathers information on extraordinary supports an individual needs in terms of Frequency, Time, and Type in six major daily living areas. Section 1 is made up of six subscales: Home Living, Community Living, Lifelong Learning, Employment, Health \& Safety, and Social activities. The six subscales provide raw scores that are converted to subscale standard scores and subscale percentiles to provide a Support Needs Profile. The subscale standard scores are also combined to produce a composite standard score, Support Needs Index (SNI). Section 2 Supplemental Protection \& Advocacy (P\&A) Scale, also requires ratings of Frequency, Time, and Type in regard to activities of daily life, but no standard scores are generated. Section 3 Exceptional Medical and Behavioral Support Needs requires ratings of an individual's extraordinary support needs because of medical conditions and challenging behaviors. This section uses a 3-point Likert scale.

Thompson et al. (in press), however, reported the P\&A scale was a psychometrically robust measure of support needs. When the SIS is revised, it is highly likely that the P\&A scale will be used to produce a standard subscale score and contribute to the composite SNI score. Therefore, the revised version of SIS will include two sections: Section 1 composing seven subscales (i.e., Home Living, Community Living, Lifelong Learning, Employment, Health \& Safety, Employment, and P\&A) and Section 2 Extraordinary Medical and Behavioral Support Needs (J. R. Thompson, personal communication, November 1, 2013). Thus, the new SNI score will include data from the P\&A subscale. For purposes of clarity, the P\&A supplemental scale will be referred to as the P\&A subscale from this point forward in this dissertation. Also, it should be assumed that any references to SIS subscales or subscale scores include the P\&A scale unless otherwise indicated. 
In the SIS Users Manual, Thompson et al. (2004b) reported robust psychometric properties for the SIS. Internal consistency coefficients ranged from 0.86 to 0.99 , inter-rater reliability coefficients ranged from 0.35 to 0.79 , test-retest reliability coefficients ranged from 0.52 to 0.82 , and the SIS had adequate concurrent validity with the Inventory for Client and Agency Planning (ICAP; coefficients ranged from -0.23 to -0.68). In 2008, Thompson et al. investigated the inter-interviewer reliability, inter-respondent reliability, and mixed inter-rater reliability of the SIS when interviewers had completed a training session or had been familiar with the SIS. They reported much stronger inter-rater reliability (subscale coefficients ranged from 0.51 to 0.90 , and composite score coefficients ranged from 0.85 to 0.90 ) than were reported in the SIS Users Manual.

The SIS was normed with 1,306 adults with ID/DD. This sample size was sufficient to allow users to compare an individual's support needs level with a standardization sample that is representative of adults served by the ID/DD agencies. Also, the scale's translated versions have been investigated by researchers for over a decade and have shown strong statistical properties. Actually, the psychometric findings of translated versions have been very consistent with those reported for the original English version by Thompson et al. (2004b; e.g., see Chou et al., 2013; Claes et al., 2009; Cruz et al., 2013; Giné, 2008; Janero et al., 2011; Kuppens et al., 2010; Lamoureux-Hebert \& Morin, 2008; Smit et al., 2011; Verdugo et al., 2006; Weiss et al., 2009).

Fortune et al. (2009) have analyzed SIS data for the purpose of developing funding algorithm recommendations for multiple jurisdictions, including several U.S. states and European countries. They reported that although Sections 2 and 3 do not provide standard scores, these sections were useful for understanding an individual's support needs and developing a support plan.

Despite the benefits of Section 3 of the SIS (i.e., Exceptional Medical and Behavioral Support Needs), however, scores from this section were not used in the analyses associated with 
this study because of several reasons. The Exceptional Medical and Behavioral Support Needs scale was qualitatively different than the seven SIS subscales. Whereas the seven subscales focus on intensity of supports needed to participate in valued life activities, Section 3 focuses on supports needed to cope with a medical or behavioral problem. For example, one of the Behavioral Support Needs items concerns "tantrumming." This score does not measure how much support is needed to participate in tantrumming, but rather, how much extra support is needed to assist the person when he or she is having a tantrum. Furthermore, consideration of additional support is required because of medical conditions and behavioral problems are embedded in the seven subscales. For example, if a person is suicidal, that person will need close monitoring during every life activity regardless of other types of supports needed to participate in various activities. Also, the variance in scores on Extraordinary Medical and Behavioral Support Needs is simply too small, and therefore, the scores would unlikely be useful in grouping the participants into more than two clusters.

\section{Statistical Measures}

To complete cluster analysis and CFA procedures on CW data provided by the HSRI, steps to complete the investigation are described below.

Step 1 Data cleaning and preparation. SIS item scores were calculated by summing scores of frequency, time, and type together (as is called for in the SIS Users Manual; Thompson et al., 2004b). Then, item scores within each subscale were summed to generate SIS subscale scores (as is also called for in the SIS Users Manual). Raw scores were not converted to standard scores, because there are no standard score conversions for the P\&A subscale. Moreover, using standard scores is not required when completing cluster analysis procedures (Bailey, 1994; Lattin et al., 2003). Data were checked for missing values. Cases with missing data on one or more of the seven SIS subscales were deleted. A codebook was created to delineate all variables and possible values. 
Step 2 Investigating descriptive analysis and reliability of data. Descriptive statistics (e.g., mean, standard deviation) were generated to summarize characteristics of the data set and compare the data set of Colorado used in this dissertation to other states' data. Descriptive statistics were conducted to identify any ways in which the people on whom data were collected in this investigation might be systematically different in important characteristics than people whose characteristics were reported in other large data sets who were considered representative of the population of people with ID/DD in the U.S. The HSRI has provided demographic data from other states with whom they have worked, and these are detailed in a variety of reports (e.g., Agosta et al., 2009; Kimmich et al., 2009). Also, Braddock et al. (2013) was consulted, as they have collected state and national data for over 30 years. Finally, the characteristics for the standardization sample on which the SIS was normed were reviewed. Cronbach's alpha was used to examine the internal consistency of the data set.

Step 3 Examining appropriate number of clusters. Lattin et al. (2003) stressed the importance of running cluster analysis several times with different starting points to ensure a good clustering model. The starting points are usually called predetermined numbers (Ks) of clusters. They also recommended using multiple clustering techniques to find the $K$ s as different techniques have relative strengths. For this study, the Complete Linkage method, Ward's method, and user-defined seeds were used to identify the Ks.

The first method, Complete Linkage, joins two clusters together by considering the distance between the farthest pair of objects in the clusters. Because the Complete Linkage is highly sensitive to outliers, all objects in each cluster are close to each other (Lattin et al., 2003). This aligns with the purpose of this dissertation; namely, to produce homogeneous groupings of people with ID/DD. The second technique, Ward's method, focuses on joining two clusters whose merger provides the smallest within-cluster variance. As a result, this method minimizes within-group distances (Lattin et al., 2003) which is the purpose of this study. Applying these 
two methods using SPSS 21 statistical program generated clusters of people with ID/DD that have maximum between-group differences. For the user-defined seeds (i.e., set of cluster seeds based on an expert's or the analyst's knowledge and expertise; Lattin et al., 2003), the SIS lead author (i.e., Dr. James R. Thompson) and literature in the field were consulted to identify $\mathrm{Ks}$ based on his experiences and expertise.

With the Ks derived from different techniques, $K$-means cluster analysis was used to classify the waiver population into $K$ groups based on their support needs, personal characteristics, and other information (e.g., residential settings). $K$-means is a method that divides the participants into $K$ non-overlapping groups so that the participants in each cluster are relatively homogeneous and people in different groups are relatively heterogeneous.

Step 4 Finding latent factor model of support needs. Using LISREL 8.8 (Jöreskog \& Sörbom, 2006), data were analyzed to find a latent factor model that best fit the support needs data collected on CW participants. First, the data set was randomly divided into Model Development and Model Testing groups. CFA was used on the Model Development sample to investigate whether data from that group supported one of the previously suggested models. If data did not confirm any of the models, EFA would be employed to find the best model.

Bollen and Long (1993) suggested five steps for researchers conducting CFA: specification, identification, estimation, assessment of model fit, and respecification. Model specification means specifying the initial model(s) that the researcher is interested in and, in this case, the initial models are all based on latent factor structures proposed from prior research studies or conceptual writing about the nature of people's support needs that has been published in the professional literature (see the Research Questions portion of this chapter for references providing justification for each model). The five competing models are shown in Appendix A (i.e., a 7-factor model, a 7-factor model with a higher-order factor, a 5-factor model based on a subset of SIS items, a 5-factor models based on all SIS items, and a unidimensional, single-factor 
model).

Model identification involves finding whether parameters are uniquely determined for each model (Long, 1983). If they are, then the next step is model estimation. For this investigation, the five models were estimated using the Maximum Likelihood method. The final two steps are assessment of model fit and model respecification. The models were tested using multiple goodness-of-fit statistics (i.e., Root-Mean-Square error of approximation or RMSEA, Standardized-Root-Mean-Square Residual or S-RMR, Non-Normed Fit Index or NNFI, and Comparative Fit Index or CFI; Hu \& Bentler, 1999; Tanaka, 1993). Then the results from the goodness-of-fit tests were interpreted to identify the best fitting model and determine the need for model respecification.

In validating the model, goodness-of-fit statistics (i.e., RMSEA, S-RMR, NNFI, and CFI) were conducted again on the Model Testing sample to find whether the group yielded positive results as on the Model Development sample. If the second data set yielded similar findings as the Model Development group, it would be appropriate to conclude that the best fitting model was validated.

\section{Chapter Summary}

In this chapter, details were provided in regard to the procedures used to answer the research questions guiding this investigation. The cluster analysis procedures that were used to identify groupings of people with ID/DD were explained. Also, the CFA procedures to identify the most plausible factor structure to explain the support needs data were provided. In the next chapter, findings that resulted from implementing these procedures will be presented. 


\section{CHAPTER IV}

\section{ANALYSIS OF DATA}

\section{Statement of the Problem}

Supports and support needs have been a focus of researchers in the field of intellectual and related developmental disabilities (ID/DD) over the last two decades. A better understanding of the nature of support needs could lead to improved supports and more responsive services for this population, enhanced personal outcomes, and a higher quality of life. Although progress has been made in understanding people with ID/DD by support needs, no systematic analysis of large-scale support needs data has been completed to investigate ways of classifying people with ID/DD by support needs. In this study, individuals with ID/DD in Colorado were grouped into homogeneous clusters using a variety of clustering techniques. Also, the construct of support needs was investigated by examining the factor structure of the Supports Intensity Scale (SIS). Confirmatory factor analysis (CFA) of a large data set was completed to study the extent of empirical support for alternative latent factor models of the support needs of people with ID/DD.

\section{Research Questions}

The following research questions have guided this study:

1. What multiple grouping model, based on differing support needs, is generated from a statewide sample of people with ID/DD using cluster analysis?

2. Based on CFA, which of the following latent factor models of support needs proposed in prior professional literature is best supported by data from a statewide sample of people with ID/DD: a 7-factor model aligned with the SIS subscale structure, a 7-factor hierarchical model, a 5-factor model using a subset of SIS items, a 5-factor model with all SIS items, or a single- 
factor model? (See Appendix A)

\section{Procedures and Findings}

Due to the nature of this investigation, decisions regarding certain procedures needed to be made following different stages of analysis. In this chapter, findings are not only presented according to the data analysis procedures described in Chapter 3, but procedures and findings from additional analyses are also presented. There are four sections in this chapter. Data Cleaning and Preparation explains how data used in this study were adjusted before the analyses. In Investigating Descriptive Analysis and Reliability of Data, important demographic information of Comprehensive Home and Community-Based Services Waiver Program (CW) participants in Colorado (i.e., on whom data used in this study were collected) are compared to national data, other states, and the SIS standardization sample. Also, information regarding the reliability of $\mathrm{CW}$ data is reported. The Examining Appropriate Number of Clusters section includes descriptions of different techniques used to cluster CW participants with ID/DD in Colorado into homogeneous groups based on support needs. Descriptions of proposed clusters are also presented. The last part of this chapter, Finding the Latent Factor Model of Support Needs of Best Fit, focuses on results from factor analysis techniques to investigate which latent factor model of support needs is best supported by data used in this investigation.

\section{Data Cleaning and Preparation}

CW data used in this dissertation, provided by the Human Services Research Institute (HSRI), were a result of their resource allocation work with the state of Colorado. These data were evaluated for missing values. Out of 4,003 cases in the CW data set, two cases did not provide item scores for Protection \& Advocacy (P\&A) activities and Extraordinary Medical and Behavioral Support Needs. Three cases did not provide any data. Therefore, five cases were deleted, and the $\mathrm{CW}$ data set included data from 3,998 participants. Although the data set came with individuals' IQ scores, a state authority indicated that the IQ scores were of questionable 
validity (J. Fortune, personal communication, August 22, 2013). Moreover, IQ scores of 795 participants were coded as -9 and IQ scores of 187 people were missing. Therefore, IQ scores were not used in analyses associated with this study.

The SIS item scores and subscale scores were calculated from scores of frequency, time, and type. As noted in Chapter 3, however, the Extraordinary Medical and Behavioral Support Needs items in Section 3 of the SIS were excluded from analyses in this study because these items focused on supports needed to successfully cope with medical and behavioral problems and did not measure supports needed to participate in life activities (like the items in the subscales). Therefore, SIS data used in this study composed 57 items from the seven subscales.

\section{Investigating Descriptive Analysis and Reliability of Data}

The 3,998 adults in this study included 2,350 men (58.8\%) and 1,648 women (41.2\%). Their age ranged from 17 to $96(\mathrm{M}=43.33, \mathrm{SD}=13.59)$. Based on Colorado caseworker ratings of community risk, the vast majority of participants presented no special risk (3,726 individuals, 93.2\%), 193 participants (4.8\%) needed supervision but did not have serious risks, and 79 individuals $(2.0 \%)$ had significant community safety risks due to histories of encounters with the criminal justice system. The number of $\mathrm{CW}$ participants in six funding groups, based on funding levels HSRI established for grouping people with ID/DD in Colorado, is shown in Table 5. 
Table 5

Colorado's Funding Levels Suggested by the HSRI

\begin{tabular}{ccccccc}
\hline $\begin{array}{c}\text { Funding } \\
\text { level }\end{array}$ & $\begin{array}{c}\text { \# of CW } \\
\text { participants }\end{array}$ & $\begin{array}{c}\text { Spending } \\
\text { (Mean) }\end{array}$ & $\begin{array}{c}\text { SIS ABE } \\
\text { raw scores }\end{array}$ & $\begin{array}{c}\text { SIS ABE } \\
\text { percentile }\end{array}$ & SIS MS & SIS BS \\
\hline 1 & $1,069(26.7 \%)$ & $\$ 10,818$ & $\leq 30$ & $\leq 50^{\text {th }}$ & Not used & $\leq 6$ \\
2 & $640(16.0 \%)$ & $\$ 14,867$ & $\geq 31$ to $\leq 36$ & $51^{\text {st }}$ to $75^{\text {th }}$ & Not used & $\leq 9$ \\
3 & $643(16.1 \%)$ & $\$ 18,040$ & $\geq 37$ & $\geq 76^{\text {th }}$ & Not used & $\leq 10$ \\
4 & $597(14.9 \%)$ & $\$ 18,173$ & $\geq 12$ to $\leq 21$ & $33^{\text {rd }}$ to $60^{\text {th }}$ & Not used & 7 to 10 \\
5 & $645(16.1 \%)$ & $\$ 18,821$ & Not used & Not used & $\geq 8$ & Not used \\
6 & $404(10.1 \%)$ & $\$ 18,752$ & Not used & Not used & Not used & $\geq 11$ \\
\hline
\end{tabular}

Note. SIS ABE = Scores on Home Living, Community Living, and Health \& Safety subscales; SIS MS = Exceptional Medical Support Needs; SIS BS = Exceptional Behavioral Support Needs

SIS raw scores from each subscale varied: Home Living $(\mathrm{M}=49.92, \mathrm{SD}=18.07)$, Community Living $(\mathrm{M}=53.60, \mathrm{SD}=12.58)$, Lifelong Learning $(\mathrm{M}=66.02, \mathrm{SD}=12.11)$, Employment $(M=57.58, S D=13.46)$, Health \& Safety $(M=53.47, S D=13.27)$, Social Activities $(\mathrm{M}=51.25, \mathrm{SD}=15.03)$, and Protection \& Advocacy $(\mathrm{M}=54.90, \mathrm{SD}=12.47)$. As mentioned in Chapter 3, the mean raw scores of Extraordinary Medical $(\mathrm{M}=3.04, \mathrm{SD}=3.24)$ and Behavioral Support Needs $(\mathrm{M}=6.38, \mathrm{SD}=4.85)$ were very small due to the use of a 3-point rating scale for each item, and the fact that by definition, the medical and behavioral conditions were uncommon (e.g., it is likely that only a few people would be suicidal). The Support Needs Index (SNI) ranged from 59 to $143(\mathrm{M}=100.41, \mathrm{SD}=10.87)$.

To judge whether participants in the $\mathrm{CW}$ data set were representative of a population of people with ID/DD in the U.S., data from CW participants were compared to statewide and national data reported by Braddock et al. (2013). Through the State of the States (2013) project, Braddock and his colleagues have collected data since 1977. Their 35-year longitudinal database covers an extensive array of variables relevant to the field of ID/DD (e.g., community living, public and private residential settings, family support, supported employment, supported living, 
Medicaid Waivers, demographics).

Braddock et al. (2013) reported that among 613,184 people with ID/DD in the U.S. who lived in out-of-home residential settings in $2011,472,334$ people $(76 \%)$ lived in small residential settings (for 1-6 residents), 56,418 people (9\%) lived in midsized settings (for 7-15 residents), and 84,432 people (14\%) lived in large institutions (for 16 residents or more). They also reported that 8,420 people (93\%), 141 people (2\%), and 434 people $(5 \%)$ in Colorado lived in small, medium, and large settings respectively. In the $\mathrm{CW}$ data set, it is evident that a majority of people (63.5\%) lived in small living arrangements (for 1-6 residents), 13.8\% lived in midsized residential settings for 7-8 residents, and 22.6\% lived in other Individual Residential Services and Supports (IRSS; other types of residential settings than group homes and host homes such as foster care homes without the formal foster case relationship). Although precise numbers of housemates in IRSS are unknown, the numbers are typically very small in such arrangements. Residence was not reported for six people $(0.1 \%)$. Although proportions of people living in different-sized residential alternatives in the $\mathrm{CW}$ dataset were slightly different than the national and statewide data reported by Braddock et al., it is apparent that the $\mathrm{CW}$ data set was relatively well aligned with Braddock et al.'s data in regard to residential setting. This finding suggests that people in the $\mathrm{CW}$ data set are generally representative of people with ID/DD in the U.S. in terms of living arrangements based on number of housemates.

SIS scores and demographic characteristics of people in the CW data set were compared to other states that HSRI has worked with, as well as the SIS standardization sample. HSRI, who has provided consultation on resource allocation to a number of states, reported that the distribution of SNI scores of CW participants was very similar to other states (e.g., Georgia, Oregon, Virginia; Kimmich et al., 2009). SNI score distribution in all the states has shown a normal distribution (i.e., bell-shaped curve) as is indicated in the SIS Users Manual. Furthermore, the CW participants had similar demographic characteristics (e.g., gender, age) to 
those reported in the SIS Users Manual (Thompson et al., 2004b). Their findings, in conjunction with the comparisons to Braddock et al.'s (2013) data, strongly suggest that the CW data set provides a representative sample for investigating adults with ID/DD.

Reliability. Fifty-seven item scores of the seven SIS subscales from 3,998 CW participants were analyzed to examine reliability (i.e., internal consistency) of SIS scores. Cronbach's alpha of $\mathrm{CW}$ data equaled 0.98 , revealing that $\mathrm{CW}$ data used in this study have high internal consistency. The degree of internal consistency is consistent with findings reported in SIS Users Manual (Thompson et al., 2004b). This indicates that SIS data used in this investigation are reliable.

\section{Examining Appropriate Number of Clusters}

SIS item scores, subscale scores, and composite scores supply different information to serve different purposes. Item scores identify specific intensities of supports needed by people in daily life activities. Subscale scores indicate supports needed in different life activity categories. A composite score, the sum of six SIS subscale scores, can be converted into a SNI score. The SNI score allows a comparison of an individual's overall support needs and people in the standardization sample. Item scores, subscale scores, and the SNI score provide a useful overview of a person's pattern of support needs.

Identifying predetermined numbers of clusters. To find predetermined numbers of cluster $(K \mathrm{~s})$, several clustering techniques were conducted on the SIS item scores of 3,998 CW participants in Colorado. The Complete Linkage method and Ward's method were used, and both methods divided the participants into two clusters based on agglomeration schedules and dendrograms. User's defined seeds were also used in finding initial numbers of cluster. In 1992, the AAIDD proposed the ILEP classification system that classified people with ID/DD into four groups based on intensity of support needs (Luckasson et al., 1992). The lead author of the SIS suggested at least three clusters of people with ID/DD were needed when grouping by support 
intensity due to heterogeneity of the ID/DD population (J. R. Thompson, personal communication, August 1, 2013). Historically, classification typologies of ID/DD have not gone below three groupings and rarely beyond five groupings (Scheerenberger, 1983). Therefore, user's defined seeds were three, four, and five clusters, and thus 2-, 3-, 4-, and 5-cluster solutions were investigated using $K$-means clustering technique.

Testing for the best cluster solution. In this step, subscale scores were used instead of item scores because subscale scores provided an effective data reduction of support needs. $K$ means was conducted to divide people with ID/DD into predetermined numbers of relatively homogeneous clusters; so, members in different groups were relatively heterogeneous based on SIS subscale scores. After independent and dependent variables were specified, $K$-means yielded cluster membership (each participant was assigned to a cluster). The cluster membership of the 2-, 3-, 4-, and 5-cluster solutions were used to calculate and compare means of cluster centroids using Multivariate Analysis of Variances (MANOVAs), Scheffe post-hoc tests, and $t$-tests. The solutions were evaluated to determine if there were adequate distinctions between groups. In addition, findings were examined to determine the relative functionality of different solutions (i.e., to see which solution was most functional). Finally, and most importantly, the solutions were evaluated in regard to interpretability.

Comparing cluster solutions $\mathbf{- 2}$ clusters. Keeping cluster membership of the 2-cluster solution as a fixed factor and the seven SIS subscale scores as dependent variables, MANOVA revealed that means of the two centroids were significantly different: Wilks' $\Lambda=.31, F(7,3990)$ $=1,255.51, p<.001, \eta_{p}^{2}=.69$. Data showed that effect size of the solution was significantly strong. In Table 6, findings of univariate tests are presented and rank-ordered by Partial-EtaSquared values. These findings suggest that the most significant difference in the 2-cluster solution was due to Home Living $\left(\eta_{p}^{2}=.59\right)$, followed by Health \& Safety $\left(\eta_{p}^{2}=.57\right)$, Employment $\left(\eta_{p}^{2}=.54\right)$, Social Activities $\left(\eta_{p}^{2}=.51\right)$, Protection \& Advocacy and Lifelong 
Learning $\left(\eta_{p}^{2}=.50\right)$, and Community Living $\left(\eta_{p}^{2}=.49\right)$.

Table 6

Results of the Univariate ANOVAs of SIS Scores in the 2-Cluster Solution

\begin{tabular}{lccccc}
\hline \multicolumn{1}{c}{ SIS scores } & $\mathrm{R}^{2}$ & $\mathrm{df}$ & $\mathrm{M}^{2}$ & $\mathrm{~F}$ & $\eta_{p}^{2}$ \\
\hline Home Living*** & .59 & 1 & 774849.82 & 5832.36 & .59 \\
Health \& Safety*** & .57 & 1 & 403809.22 & 5370.29 & .57 \\
Employment*** & .54 & 1 & 394185.70 & 4779.70 & .54 \\
Social Activities*** & .51 & 1 & 463960.16 & 4224.04 & .51 \\
Protection \& Advocacy*** & .50 & 1 & 309295.75 & 3959.20 & .50 \\
Lifelong Learning*** & .50 & 1 & 290971.80 & 3937.49 & .50 \\
Community Living*** & .49 & 1 & 311206.45 & 3872.92 & .49 \\
\hline
\end{tabular}

Note. $* * * p<.001$

Personal information of the participants (nominal data) was examined using crosstabulations and Pearson Chi-Square tests. Relationship between cluster membership and community safety risk was highly significant: $\chi^{2}(2, N=3998)=39.90, p<.001$. In regard to the funding level that the state established for individuals, cluster membership and CW funding level had a highly significant relationship: $\chi^{2}(5, N=3998)=1233.08, p<.001$. Type of living arrangement was also significantly related to cluster membership: $\chi^{2}(7, N=3998)=407.52$, $p<.001$. Use of habilitation $\left(\chi^{2}[1, N=3997]=145.88, p<.001\right)$, use of community services $\left(\chi^{2}[1, N=3997]=135.75, p<.001\right)$, use of sheltered employment $\left(\chi^{2}[1, N=3997]=81.22\right.$, $p<.001)$, use of supported employment $\left(\chi^{2}[1, N=3997]=202.64, p<.001\right)$, and use of individual employment $\left(\chi^{2}[1, N=3997]=464.36, p<.001\right)$ were also significantly related to cluster membership. Finally, although age and cluster membership had a significant relationship $\left(\chi^{2}[74, N=3948]=105.08, p=.01\right)$, language and gender did not.

Although the 2-cluster solution was statistically significant, this model was not satisfying in terms of understanding the population of people with ID/DD. A dichotomous model was not 
useful for practical purposes because the differentiation is so broad. People with ID/DD are a heterogeneous population, and having only two broad groups of people with ID/DD is not useful for the practical purposes of sharing information, planning, or developing public policies.

Comparing cluster solutions $\mathbf{- 3}$ clusters. Using cluster membership of the 3 -cluster solution as the fixed factor and the SIS subscale scores as dependent variables, results derived from MANOVA showed that the 3-cluster solution was also significant: Wilks' $\Lambda=.15, F(14$, 7978) $=897.45, p<.001, \eta_{p}^{2}=.61$. In Table 7 , all subscales had relatively strong relationship (ranging from .69 in Health \& Safety to .62 in Protection \& Advocacy). Community safety risk $\left(\chi^{2}[4, N=3998]=53.87, p<.001\right), \mathrm{CW}$ funding level $\left(\chi^{2}[10, N=3998]=1770.87, p<.001\right)$, type of living arrangement $\left(\chi^{2}[14, N=3998]=615.67, p<.001\right)$, use of habilitation $\left(\chi^{2}[2, N=\right.$ $3997]=213.45, p<.001)$, use of community services $\left(\chi^{2}[2, N=3997]=140.37, p<.001\right)$, use of sheltered employment $\quad\left(\chi^{2}[2, N=3997]=133.90, p<.001\right)$, use of supported employment $\left(\chi^{2}[2, N=3997]=185.03, p<.001\right)$, and use of individual employment $\left(\chi^{2}[2, N=3997]=\right.$ $5551.12, p<.001)$ significantly contributed to the cluster membership as well. Language, age, and gender, however, were not significantly related to cluster membership.

Table 7

Results of the Univariate ANOVAs of SIS Scores in the 3-Cluster Solution

\begin{tabular}{lccccc}
\hline \multicolumn{1}{c}{ SIS Subscale } & $\mathrm{R}^{2}$ & $\mathrm{df}$ & $\mathrm{M}^{2}$ & $\mathrm{~F}$ & $\eta_{p}^{2}$ \\
\hline Health \& Safety*** & .69 & 2 & 244513.86 & 4538.06 & .69 \\
Home Living*** & .69 & 2 & 452750.33 & 4519.23 & .69 \\
Social Activities*** & .66 & 2 & 299612.79 & 3941.93 & .66 \\
Employment*** & .66 & 2 & 240115.36 & 3939.34 & .66 \\
Community Living*** & .66 & 2 & 208098.50 & 3846.97 & .66 \\
Lifelong Learning*** & .64 & 2 & 186955.09 & 3517.12 & .64 \\
Protection \& Advocacy*** & .62 & 2 & 191352.26 & 3201.74 & .62 \\
\hline
\end{tabular}


Comparing cluster solutions $\mathbf{- 4}$ clusters. Conducting MANOVA on the 4 -cluster solution also yielded significant findings: Wilks' $\Lambda=.08, F(21,11451.93)=746.96, p<.001$, $\eta_{p}^{2}=.56$. Again, all subscales had a significant relationship (ranging from .76 in Health \& Safety to .67 in Protection \& Advocacy; Table 8). Data related to personal information in the four clusters was also significantly different with the exception of language, gender, and age.

Table 8

Results of the Univariate ANOVAs of SIS Scores in the 4-Cluster Solution

\begin{tabular}{lccccc}
\hline \multicolumn{1}{c}{ SIS Subscale } & $\mathrm{R}^{2}$ & $\mathrm{df}$ & $\mathrm{M}^{2}$ & $\mathrm{~F}$ & $\eta_{p}^{2}$ \\
\hline Health \& Safety*** & .76 & 3 & 178420.63 & 4216.16 & .76 \\
Home Living*** & .74 & 3 & 323932.99 & 3874.39 & .74 \\
Social Activities*** & .72 & 3 & 217999.64 & 3498.53 & .72 \\
Community Living*** & .72 & 3 & 152002.98 & 3443.68 & .72 \\
Employment*** & .71 & 3 & 171892.56 & 3299.70 & .71 \\
Lifelong Learning*** & .69 & 3 & 135255.81 & 2992.86 & .69 \\
Protection \& Advocacy*** & .67 & 3 & 138818.36 & 2704.44 & .67 \\
\hline Note. *** $p<.001$ & & & & &
\end{tabular}

Note. ${ }^{* * *} p<.001$

Comparing cluster solutions $\mathbf{- 5}$ clusters. Finally, the 5-cluster solution was tested, and its cluster centroids significantly differed as well: Wilks' $\Lambda=.06, F(28,14376.76)=625.95$, $p<.001, \eta_{p}^{2}=.51$. Similar to the 3 - and 4-cluster solutions, Table 9 shows that Health \& Safety $\left(\eta_{p}^{2}=.79\right)$ had the strongest relationship. All subscales, however, had a strong relationship $\left(\mathrm{R}^{2}\right.$ varied between .70 and .79 ). 
Table 9

Results of the Univariate ANOVAs of SIS Scores in the 5-Cluster Solution

\begin{tabular}{lccccc}
\hline \multicolumn{1}{c}{ SIS Subscale } & $\mathrm{R}^{2}$ & $\mathrm{df}$ & $\mathrm{M}^{2}$ & $\mathrm{~F}$ & $\eta_{p}^{2}$ \\
\hline Health \& Safety*** & .79 & 4 & 138509.44 & 3681.15 & .79 \\
Community Living*** & .76 & 4 & 120472.59 & 3198.19 & .76 \\
Home Living*** & .76 & 4 & 248208.17 & 3167.46 & .76 \\
Social Activities*** & .75 & 4 & 169775.17 & 3029.48 & .75 \\
Employment*** & .73 & 4 & 131723.31 & 2672.00 & .73 \\
Lifelong Learning*** & .72 & 4 & 105234.10 & 2541.57 & .72 \\
Protection \& Advocacy*** & .70 & 4 & 108675.81 & 2323.50 & .70 \\
\hline Note. $* * * p<.001$ & & & & &
\end{tabular}

Note. ${ }^{* * *} p<.001$

Cross-tabulations and Pearson Chi-Square tests were used to investigate relationship between personal information data and membership consistent with the other cluster solutions, all variables significantly contributed to cluster membership except language, age, and gender of the participants. Details are presented in Table 10. Community safety risk and cluster membership were found to be significantly related: $\chi^{2}(8, N=3998)=73.06, p<.001$. Participants who had significant community safety risks were overrepresented in Clusters 2 and 3 whose members needed relatively less supports, and underrepresented in Clusters 4 and 5 whose members need relatively more intense supports. This means that Clusters $2(34.2 \%)$, and $3(38.0 \%)$ tended to have had more people who were convicted of criminal activities in comparison to Clusters 4 $(15.2 \%)$ and $5(2.5 \%)$. Therefore, although people in Clusters 2, and 3 had relatively less intense support needs in comparison to those in Clusters 4 and 5, they were more likely to become involved with the criminal justice system. Put another way, people in Clusters 4 and 5 required the highest intensity of support to participate in daily life activities, but they posed less community safety risks than other cluster members as measured by history of criminal activity. Cluster membership was also significantly related with the funding levels derived by 
HSRI for people with ID/DD in the state of Colorado $\left(\chi^{2}[20, N=3998]=2223.66, p<.001\right)$ and type of living arrangement $\left(\chi^{2}[28, N=3998]=758.48, p<.001\right)$. People receiving CW Funding Level 1 (i.e., the least spending) were overrepresented in the two clusters associated with relatively less intense support needs (i.e., Clusters 1 and 2). Conversely, most people in Clusters 4 and 5 received greater amounts of public funding (i.e., Levels $3-6$ ). In regard to type of living arrangement, people who had more intense support needs (i.e., Clusters 3, 4, and 5) tended to live more in host homes or group homes. More people with less intense support needs (i.e., Clusters 1 and 2) lived in other IRSS (i.e., foster care homes without formal foster care relationship).

The 5-cluster solution - the model of best fit. The 5-cluster model was selected because it yielded maximum clusters with statistically significant centroid differences (i.e., $p<.001)$ for the seven SIS subscales of support needs on which data were collected. Table 11 reveals that cluster membership is based on relative intensity of supports that people need. Cluster 1 composes 313 individuals (7.8\%) who have the lowest support needs in all seven subscales. Cluster 2 is a group of 660 people (16.5\%) who have relatively low support needs but higher than Cluster 1. In Cluster 3, mean raw scores of 893 members (22.3\%) are in the middle. Cluster 4 is the largest group of CW participants in Colorado $(n=1,165 ; 29.1 \%)$. The mean support needs scores for these participants are relatively high. The last group of individuals is Cluster 5. These 967 participants (24.2\%) have the highest mean scores.

Most participants did not use individual employment services. Among people who used it, however, participants in Clusters 1 and 2 were overrepresented. Nearly 50\% of CW participants used community services, and most of them were in Clusters 3, 4, and 5 . Only one third used habilitation services, and those services were used by people in Clusters 4 and 5 . Although supported employment and sheltered employment services were not used by a large majority of CW participants, people in Clusters 2, 3, and 4 still used these services. Nevertheless, as mentioned before, language, gender, and age were variables that did not have any significant 
relation to group membership in the 5-cluster solution (i.e., no statistical differences between groups were noted).

Figure 1 shows a summary of the findings. Data from this investigation support grouping people by five levels of intensity in relation to seven subscales of support needs. Additionally, an overall measure of support intensity (i.e., SNI scores) further illustrates how the five groups differ in regard to the intensity of supports they need to fully participate in daily life activities in community setting.

Table 10

Personal Information of CW Participants in the 5-Cluster Solution

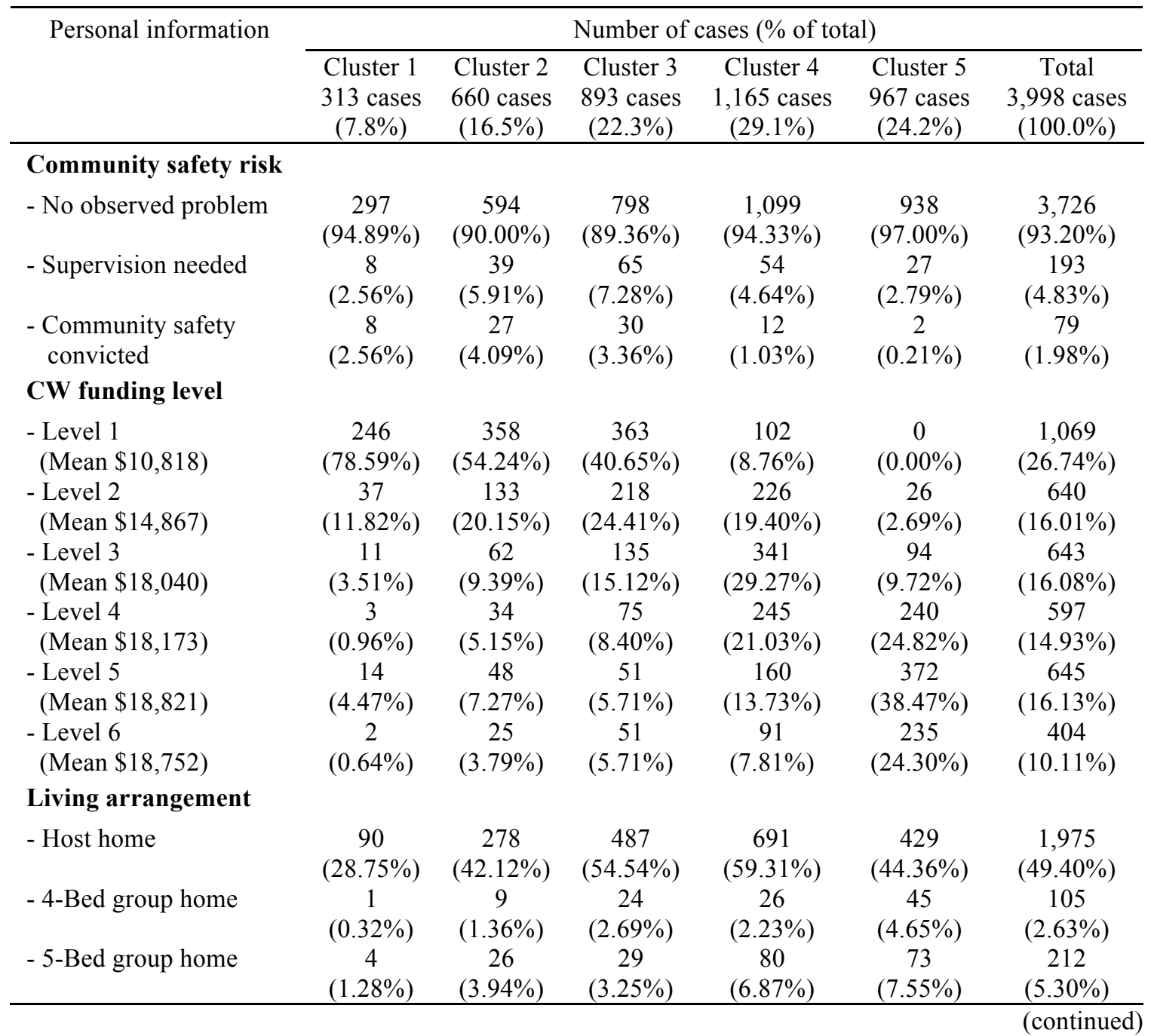


Table 10

Personal Information of $C W$ Participants in the 5-Cluster Solution (continued)

\begin{tabular}{|c|c|c|c|c|c|c|}
\hline \multirow[t]{2}{*}{ Personal information } & \multicolumn{6}{|c|}{ Number of cases ( $\%$ of total) } \\
\hline & $\begin{array}{c}\text { Cluster } 1 \\
313 \text { cases } \\
(7.8 \%) \\
\end{array}$ & $\begin{array}{c}\text { Cluster } 2 \\
660 \text { cases } \\
(16.5 \%) \\
\end{array}$ & $\begin{array}{c}\text { Cluster } 3 \\
893 \text { cases } \\
(22.3 \%) \\
\end{array}$ & $\begin{array}{c}\text { Cluster } 4 \\
1,165 \text { cases } \\
(29.1 \%) \\
\end{array}$ & $\begin{array}{c}\text { Cluster } 5 \\
967 \text { cases } \\
(24.2 \%) \\
\end{array}$ & $\begin{array}{c}\text { Total } \\
3,998 \text { cases } \\
(100.0 \%) \\
\end{array}$ \\
\hline - 6-Bed group home & $\begin{array}{c}5 \\
(1.60 \%)\end{array}$ & $\begin{array}{c}18 \\
(2.73 \%)\end{array}$ & $\begin{array}{c}46 \\
(5.15 \%)\end{array}$ & $\begin{array}{c}77 \\
(6.61 \%)\end{array}$ & $\begin{array}{c}100 \\
(10.34 \%)\end{array}$ & $\begin{array}{c}246 \\
(6.15 \%)\end{array}$ \\
\hline - 7-Bed group home & $\begin{array}{c}7 \\
(2.24 \%)\end{array}$ & $\begin{array}{c}19 \\
(2.88 \%)\end{array}$ & $\begin{array}{c}26 \\
(2.91 \%)\end{array}$ & $\begin{array}{l}54 \\
(4.64 \%)\end{array}$ & $\begin{array}{c}44 \\
(4.55 \%)\end{array}$ & $\begin{array}{c}150 \\
(3.75 \%)\end{array}$ \\
\hline - 8-Bed group home & $\begin{array}{c}9 \\
(2.88 \%)\end{array}$ & $\begin{array}{c}44 \\
(6.67 \%)\end{array}$ & $\begin{array}{c}75 \\
(8.40 \%)\end{array}$ & $\begin{array}{c}86 \\
(7.38 \%)\end{array}$ & $\begin{array}{c}190 \\
(19.65 \%)\end{array}$ & $\begin{array}{c}404 \\
(10.11 \%)\end{array}$ \\
\hline - Other IRSS & $\begin{array}{c}197 \\
(62.94 \%)\end{array}$ & $\begin{array}{c}266 \\
(40.30 \%)\end{array}$ & $\begin{array}{c}205 \\
(22.96 \%)\end{array}$ & $\begin{array}{c}151 \\
(12.96 \%)\end{array}$ & $\begin{array}{c}86 \\
(8.89 \%)\end{array}$ & $\begin{array}{c}905 \\
(22.64 \%)\end{array}$ \\
\hline - Missing & - & - & $\begin{array}{c}1 \\
(0.00 \%)\end{array}$ & & & $\begin{array}{c}1 \\
(0.00 \%)\end{array}$ \\
\hline \multicolumn{7}{|c|}{ Use of habilitation services } \\
\hline - Yes & $\begin{array}{c}50 \\
(15.97 \%)\end{array}$ & $\begin{array}{c}148 \\
(22.42 \%)\end{array}$ & $\begin{array}{c}223 \\
(24.97 \%)\end{array}$ & $\begin{array}{c}398 \\
(34.16 \%)\end{array}$ & $\begin{array}{c}516 \\
(53.36 \%)\end{array}$ & $\begin{array}{c}1,335 \\
(33.39 \%)\end{array}$ \\
\hline - No & $\begin{array}{c}263 \\
(84.03 \%)\end{array}$ & $\begin{array}{c}512 \\
(77.58 \%)\end{array}$ & $\begin{array}{c}669 \\
(74.92 \%)\end{array}$ & $\begin{array}{c}767 \\
(65.84 \%)\end{array}$ & $\begin{array}{c}451 \\
(46.64 \%)\end{array}$ & $\begin{array}{c}2,662 \\
(66.58 \%)\end{array}$ \\
\hline - Missing & & & $\begin{array}{c}1 \\
(0.00 \%)\end{array}$ & & & $\begin{array}{c}1 \\
(0.00 \%)\end{array}$ \\
\hline \multicolumn{7}{|c|}{ Use of community services } \\
\hline - Yes & $\begin{array}{c}76 \\
(24.28 \%)\end{array}$ & $\begin{array}{c}219 \\
(33.18 \%)\end{array}$ & $\begin{array}{c}391 \\
(43.78 \%)\end{array}$ & $\begin{array}{c}654 \\
(56.14 \%)\end{array}$ & $\begin{array}{c}484 \\
(50.05 \%)\end{array}$ & $\begin{array}{c}1,824 \\
(45.62 \%)\end{array}$ \\
\hline - No & $\begin{array}{c}237 \\
(75.72 \%)\end{array}$ & $\begin{array}{c}441 \\
(66.82 \%)\end{array}$ & $\begin{array}{c}501 \\
(56.10 \%)\end{array}$ & $\begin{array}{c}511 \\
(43.86 \%)\end{array}$ & $\begin{array}{c}483 \\
(49.95 \%)\end{array}$ & $\begin{array}{c}2,173 \\
(54.35 \%)\end{array}$ \\
\hline - Missing & & & $(0.00 \%)$ & & & $(0.00 \%)$ \\
\hline \multicolumn{7}{|c|}{ Use of sheltered employment } \\
\hline - Yes & $\begin{array}{c}43 \\
(13.74 \%)\end{array}$ & $\begin{array}{c}157 \\
(23.79 \%)\end{array}$ & $\begin{array}{c}244 \\
(27.32 \%)\end{array}$ & $\begin{array}{c}167 \\
(14.33 \%)\end{array}$ & $\begin{array}{c}61 \\
(6.31 \%)\end{array}$ & $\begin{array}{c}672 \\
(16.81 \%)\end{array}$ \\
\hline$-\mathrm{No}$ & $\begin{array}{c}270 \\
(86.26 \%)\end{array}$ & $\begin{array}{c}503 \\
(76.21 \%)\end{array}$ & $\begin{array}{c}648 \\
(72.56 \%)\end{array}$ & $\begin{array}{c}998 \\
(85.67 \%)\end{array}$ & $\begin{array}{c}906 \\
(93.69 \%)\end{array}$ & $\begin{array}{c}3,325 \\
(83.17 \%)\end{array}$ \\
\hline - Missing & & & $\begin{array}{c}1 \\
(0.00 \%)\end{array}$ & & & $\begin{array}{c}1 \\
(0.00 \%)\end{array}$ \\
\hline \multicolumn{7}{|c|}{ Use of supported employment group } \\
\hline - Yes & $\begin{array}{c}53 \\
(16.93 \%)\end{array}$ & $\begin{array}{c}139 \\
(21.06 \%)\end{array}$ & $\begin{array}{c}149 \\
(16.69 \%)\end{array}$ & $\begin{array}{c}60 \\
(5.15 \%)\end{array}$ & $\begin{array}{c}19 \\
(1.96 \%)\end{array}$ & $\begin{array}{c}420 \\
(10.51 \%)\end{array}$ \\
\hline$-\mathrm{No}$ & $\begin{array}{c}260 \\
(83.07 \%)\end{array}$ & $\begin{array}{c}521 \\
(78.94 \%)\end{array}$ & $\begin{array}{c}743 \\
(83.20 \%)\end{array}$ & $\begin{array}{c}1,105 \\
(94.85 \%)\end{array}$ & $\begin{array}{c}948 \\
(98.04 \%)\end{array}$ & $\begin{array}{c}3,577 \\
(89.47 \%)\end{array}$ \\
\hline - Missing & & & $\begin{array}{c}1 \\
(0.00 \%) \\
\end{array}$ & & & $\begin{array}{c}1 \\
(0.00 \%) \\
\end{array}$ \\
\hline
\end{tabular}


Table 10

Personal Information of $C W$ Participants in the 5-Cluster Solution (continued)

\begin{tabular}{lcccccc}
\hline Personal information & \multicolumn{5}{c}{ Number of cases (\% of total) } \\
\cline { 2 - 7 } & Cluster 1 & Cluster 2 & Cluster 3 & Cluster 4 & Cluster 5 & Total \\
& 313 cases & 660 cases & 893 cases & 1,165 cases & 967 cases & 3,998 cases \\
& $(7.8 \%)$ & $(16.5 \%)$ & $(22.3 \%)$ & $(29.1 \%)$ & $(24.2 \%)$ & $(100.0 \%)$ \\
\hline Use of individual employment & & & & & \\
- Yes & 149 & 168 & 111 & 57 & 14 & 499 \\
& $(47.60 \%)$ & $(25.45 \%)$ & $(12.43 \%)$ & $(4.89 \%)$ & $(1.45 \%)$ & $(12.48 \%)$ \\
- No & 164 & 492 & 781 & 1,108 & 953 & 3,498 \\
& $(52.40 \%)$ & $(74.55 \%)$ & $(87.46 \%)$ & $(95.11 \%)$ & $(98.55 \%)$ & $(87.49 \%)$ \\
- Missing & \multicolumn{7}{c}{1} \\
\end{tabular}

Table 11

SIS Subscale Scores and SNI of Colorado's CW Participants in the 5-Cluster Solution

\begin{tabular}{|c|c|c|c|c|c|c|c|}
\hline \multirow[b]{2}{*}{$\begin{array}{l}\text { Subscale } \\
\text { and SNI }\end{array}$} & \multicolumn{6}{|c|}{ Mean of cluster's centroid (SD) } & \multirow[b]{2}{*}{$\begin{array}{l}\text { Explained } \\
\% \text { of the } \\
\text { variance }\end{array}$} \\
\hline & $\begin{array}{c}\text { Cluster } 1 \\
313 \text { cases } \\
(7.8 \%)\end{array}$ & $\begin{array}{c}\text { Cluster } 2 \\
660 \text { cases } \\
(16.5 \%)\end{array}$ & $\begin{array}{c}\text { Cluster } 3 \\
893 \text { cases } \\
(22.3 \%)\end{array}$ & $\begin{array}{c}\text { Cluster } 4 \\
1,165 \text { cases } \\
(29.1 \%)\end{array}$ & $\begin{array}{c}\text { Cluster } 5 \\
967 \text { cases } \\
(24.2 \%)\end{array}$ & $\begin{array}{c}\text { Total } \\
3,998 \text { cases } \\
(100.0 \%)\end{array}$ & \\
\hline $\mathrm{HL}$ & $\begin{array}{c}18.94 \\
(11.61)\end{array}$ & $\begin{array}{c}30.63 \\
(11.61)\end{array}$ & $\begin{array}{l}43.75 \\
(9.68)\end{array}$ & $\begin{array}{l}58.76 \\
(7.50)\end{array}$ & $\begin{array}{l}68.17 \\
(5.83)\end{array}$ & $\begin{array}{c}49.92 \\
(18.07)\end{array}$ & $76.0 \%$ \\
\hline $\mathrm{CL}$ & $\begin{array}{c}24.43 \\
(10.40)\end{array}$ & $\begin{array}{l}43.53 \\
(7.94)\end{array}$ & $\begin{array}{l}52.68 \\
(5.60)\end{array}$ & $\begin{array}{l}58.72 \\
(4.66)\end{array}$ & $\begin{array}{l}64.61 \\
(4.76)\end{array}$ & $\begin{array}{c}53.60 \\
(12.58)\end{array}$ & $76.2 \%$ \\
\hline LL & $\begin{array}{c}41.95 \\
(10.45)\end{array}$ & $\begin{array}{l}55.45 \\
(6.89)\end{array}$ & $\begin{array}{l}63.74 \\
(5.76)\end{array}$ & $\begin{array}{l}70.21 \\
(5.60)\end{array}$ & $\begin{array}{l}78.09 \\
(5.92)\end{array}$ & $\begin{array}{c}66.02 \\
(12.11)\end{array}$ & $71.8 \%$ \\
\hline EMP & $\begin{array}{c}32.67 \\
(10.52)\end{array}$ & $\begin{array}{l}45.17 \\
(8.08)\end{array}$ & $\begin{array}{l}53.51 \\
(7.70)\end{array}$ & $\begin{array}{l}63.09 \\
(6.32)\end{array}$ & $\begin{array}{l}71.25 \\
(4.54)\end{array}$ & $\begin{array}{c}57.58 \\
(13.46)\end{array}$ & $72.8 \%$ \\
\hline $\mathrm{H} \& \mathrm{~S}$ & $\begin{array}{l}29.64 \\
(8.60)\end{array}$ & $\begin{array}{l}40.43 \\
(6.64)\end{array}$ & $\begin{array}{l}48.97 \\
(5.78)\end{array}$ & $\begin{array}{l}58.18 \\
(5.36)\end{array}$ & $\begin{array}{l}68.56 \\
(6.01)\end{array}$ & $\begin{array}{c}53.47 \\
(13.27)\end{array}$ & $78.7 \%$ \\
\hline $\mathrm{SOC}$ & $\begin{array}{c}21.06 \\
(11.14)\end{array}$ & $\begin{array}{l}37.71 \\
(8.86)\end{array}$ & $\begin{array}{l}48.38 \\
(7.65)\end{array}$ & $\begin{array}{l}56.25 \\
(6.35)\end{array}$ & $\begin{array}{l}66.89 \\
(5.94)\end{array}$ & $\begin{array}{c}51.25 \\
(15.03)\end{array}$ & $75.2 \%$ \\
\hline $\mathrm{P} \& \mathrm{~A}$ & $\begin{array}{l}31.60 \\
(9.45)\end{array}$ & $\begin{array}{l}43.71 \\
(7.25)\end{array}$ & $\begin{array}{l}52.35 \\
(6.87)\end{array}$ & $\begin{array}{l}58.82 \\
(5.70)\end{array}$ & $\begin{array}{l}67.70 \\
(6.77)\end{array}$ & $\begin{array}{c}54.90 \\
(12.47)\end{array}$ & $69.9 \%$ \\
\hline SNI & $\begin{array}{l}77.74 \\
(5.29)\end{array}$ & $\begin{array}{l}89.25 \\
(3.07)\end{array}$ & $\begin{array}{l}97.06 \\
(2.59)\end{array}$ & $\begin{array}{l}104.97 \\
(2.70)\end{array}$ & $\begin{array}{c}112.96 \\
(3.68)\end{array}$ & $\begin{array}{l}100.41 \\
(10.87)\end{array}$ & - \\
\hline
\end{tabular}

Note. $\mathrm{HL}=$ Home Living, $\mathrm{CL}=$ Community Living, $\mathrm{LL}=$ Lifelong Learning, $\mathrm{EMP}=$ Employment, $\mathrm{H} \& \mathrm{~S}=$ Health \& Safety, SOC $=$ Social, P\&A = Protection \& Advocacy 
Figure 1. SIS Subscale Scores and SNI Scores of the Five Clusters

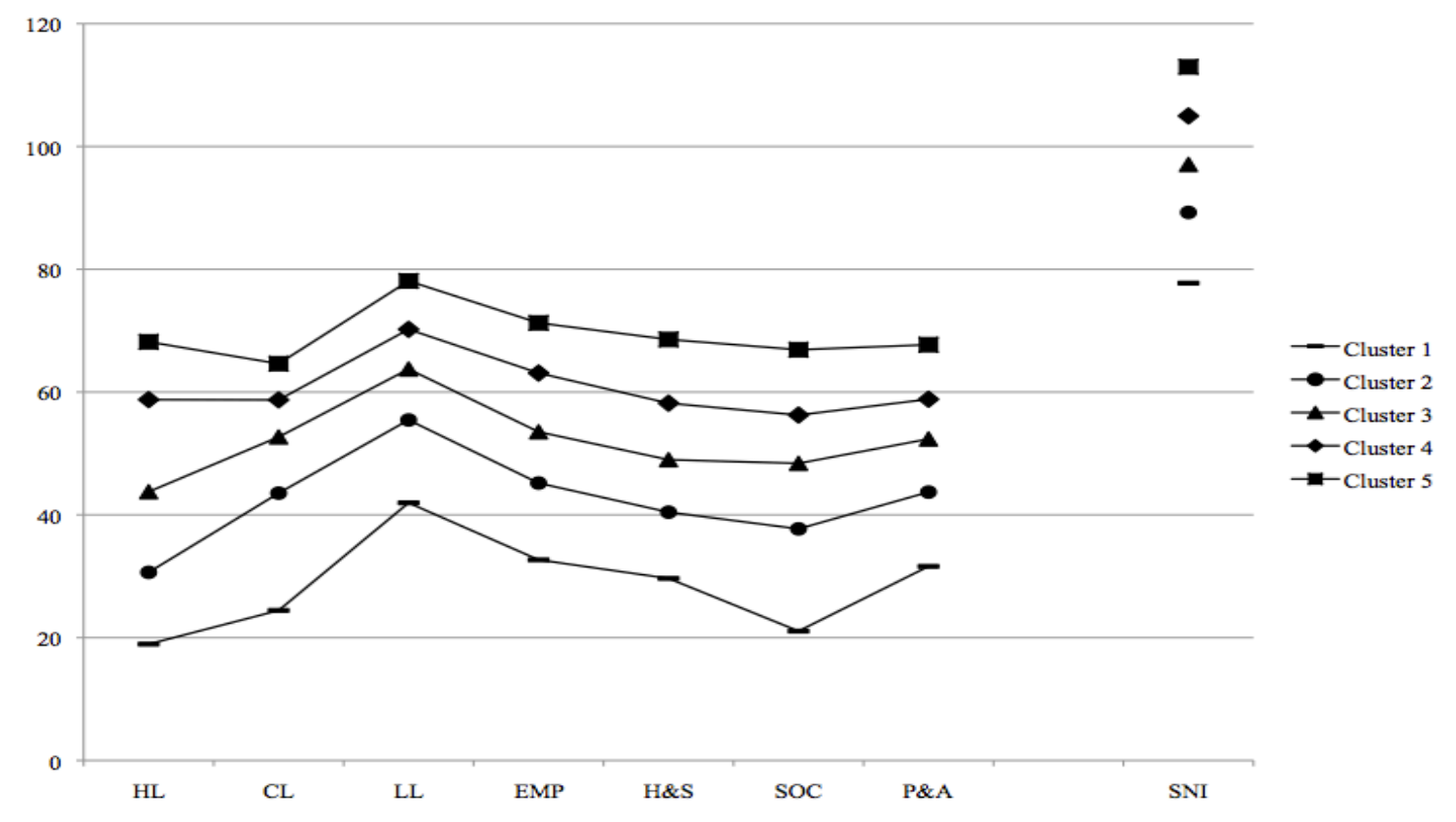

\section{Finding Latent Factor Model of Support Needs}

Researchers in the field of ID/DD have studied the support needs construct and proposed several structural models over the past decade. Although the SIS authors did not explicitly suggest a latent factor model of support needs, researchers in the field have hypothesized that support needs (at least, as measured by the SIS) consists of six latent factors aligned with the six SIS (Thompson et al., 2004a) subscales. Verdugo et al. (2006) and Kuppens et al. (2010) conducted factor analysis procedures on data they independently collected, and reported their findings supported the 6-factor, SIS subscale structure.

Other researchers, however, reported that their analysis of SIS data supported alternative factor structures. Bossaert et al. (2009) suggested a 4-factor model using 22 SIS items. Harries et al. (2005) reported that the SIS was measuring the same underlying construct as adaptive behavior scales, and they proposed a single-factor model to explain adaptive behavior skills and 
support needs.

Recently, Thompson et al. (in press) analyzed data collected through the SIS-online program as well as data collected to measure three types of inter-rater reliability (i.e., interrespondent reliability ${ }^{1}$, inter-interviewer reliability ${ }^{2}$, and mixed inter-rater reliability ${ }^{3}$ ). They reported that the P\&A supplemental scale had psychometric properties as robust as those for the six SIS subscales contributing to standard scores, and indicated that future revisions of the SIS should include the P\&A scale in the standardized score portion of the instrument.

Based on prior factor analytic research findings using SIS data, and the strong likelihood that the P\&A scale will be a subscale in future, revised versions of the SIS, five structural models were hypothesized and tested: a 7-factor model, a 7-factor hierarchical model where a higherorder latent factor connects the seven first-order factors, a 5-factor model using a subset of SIS items, a 5-factor model using all SIS items, and a unidimensional, single-factor model.

Random assignment and intercorrelations. The $\mathrm{CW}$ data set of 3,998 waiver participants was randomly divided into a Model Development group and a Model Testing group. The Random Sample of Cases function in SPSS (Version 21.0) was used to place 2,037 participants in the Model Development group and 1,961 participants in the Model Testing group. In accordance with procedures for using CFA to estimate model parameters, correlations between the seven factors were investigated using data from the Model Development group. Findings in Table 12 indicate that correlation coefficients between the seven subscales ranged from 0.73 to $0.91(p=.01)$. These findings reveal that the seven SIS subscales are moderately to strongly intercorrelated. In other words, a person who has high support needs in one subscale tends to have high support needs in other subscales, and another person who has relatively lower support needs in one subscale tends to have relatively lower support needs in other subscales.

\footnotetext{
${ }^{1}$ The same interview conducted separate assessments with different respondents

${ }^{2}$ Different interviewers completed separate assessment using the same respondents

${ }^{3}$ Different interviewers conducted assessments using different respondents
} 
Table 12

Intercorrelations Between SIS Subscales

\begin{tabular}{|c|c|c|c|c|c|c|c|}
\hline SIS subscale & HL & $\mathrm{CL}$ & LL & EMP & $\mathrm{H} \& \mathrm{~S}$ & SOC & $\mathrm{P} \& \mathrm{~A}$ \\
\hline HL & 1.00 & & & & & & \\
\hline CL & $0.81 * *$ & 1.00 & & & & & \\
\hline LL & $0.76^{* *}$ & $0.84 * *$ & 1.00 & & & & \\
\hline EMP & $0.77 * *$ & $0.78 * *$ & $0.81 * *$ & 1.00 & & & \\
\hline $\mathrm{H} \& \mathrm{~S}$ & $0.88 * *$ & $0.86^{* *}$ & $0.91 * *$ & $0.84 * *$ & 1.00 & & \\
\hline SOC & $0.73 * *$ & $0.89 * *$ & $0.84 * *$ & $0.80^{* *}$ & $0.85 * *$ & 1.00 & \\
\hline P\&A & $0.73 * *$ & $0.79 * *$ & $0.89 * *$ & $0.80^{* *}$ & $0.90 * *$ & $0.89 * *$ & 1.00 \\
\hline
\end{tabular}

The strong intercorrelations justified testing the 7-factor hierarchical model of support needs. Additionally, the presence of the SNI score, a composite measure of the overall intensity of one's support needs, suggests that the SIS was designed to measure a global, second-order factor that was related to the first-order factors aligned with the seven subscales. Therefore, in this investigation, CFA was used to evaluate the extent to data fit a 7-factor model as well as a 7factor hierarchical model.

Testing model parameters. CFA was carried out to estimate model parameters and examine whether $\mathrm{CW}$ data confirmed any of the a priori models. Five prior structural models of support needs were specified as follows:

- 7-Factor model. In this model that is aligned with the SIS subscale structure, six latent factors (i.e., Home Living, Community Living, Employment, Health \& Safety, Social Activities, and Protection \& Advocacy) each account for eight observed variables (i.e., item scores from the SIS), whereas a seventh latent factor (Lifelong Learning) accounts for nine variables.

- 7-Factor hierarchical model. This model is exactly the same as the 7-factor model in 
terms of latent factors and distribution of the corresponding 57 items (i.e., observed variables). Nevertheless, there is a higher-order latent factor that connects the seven first-order factors.

- 5-Factor model using a subset of SIS items. This model includes a subset of 30 SIS items; the 22 items identified in Bossaert et al.’s (2009) 4-factor model, plus the 8 additional items from the P\&A scale. The five latent factors are (a) Personal \& Social Skills (eight items two items from the Lifelong Learning subscale, one item from the Health \& Safety subscale, and five items from the Social Activities subscale); (b) Community Living Activities (seven items all from the Community Living subscale); (c) Daily Living Activities (four items - all from the Home Living subscale); (d) Work (three items - all from the Employment subscale); and (e) Protection \& Advocacy (eight items from the P\&A scale).

- 5-Factor model using all SIS items. This model has the same latent factor structure as the previous model. All 57 SIS items, however, are accounted for by the latent factor structure in this model. This model has 25 items on Personal \& Social Skills (25 items from the Lifelong Learning, Health \& Safety, and Social Activities subscales), Community Living Activities (eight items from the Community Living subscale), Daily Living Activities (eight items from the Home Living subscale), Work (eight items from the Employment subscale), and Protection \& Advocacy (eight items from the P\&A scale).

- Single-factor model. The model includes all 57 items from the SIS connected by a global Support Needs latent factor.

Calculating fit indices. A number of fit statistics were generated on the Model Development group using the LISREL 8.8 (Jöreskog \& Sörbom, 2006) statistical program. Maximum Likelihood Estimation was performed to estimate model parameters for each model (e.g., identify item loadings for each latent factor). Root Mean Square Error of Approximation (RMSEA) was used to investigate how well each model with unknown parameter estimates fit the data set. Conventional interpretation holds that a value less than .05 demonstrates good fit, 
whereas values from .06 to .08 yield acceptable fit, and values greater that .08 are increasingly unacceptable (Browne \& Cudeck, 1993). Standardized Root Mean Square Residual (S-RMR) is recommended when data sets are large or complex, and therefore was well aligned with the characteristics of this investigation. S-RMR provides information about standardized differences between observed correlation and predicted correlation. As with RMSEA, a value less than .05 is considered good fit, values from .06 to .08 yield acceptable fit, and values greater that .08 are increasingly unacceptable. Finally, there are two incremental fit indices that have been frequently reported by CFA researchers: the Non-normed Fit Index (NNFI) and the Comparative Fit Index (CFI). Both indices are useful in comparing a Chi-squared value of a model to a Chisquared value of a null model. A value that is .95 or higher provides an indication of a good fit, a value higher than .90 but less than .95 provides evidence of an acceptable fit, and values lower than .89 are increasingly unacceptable (Hu \& Bentler, 1999).

These four fit indices (i.e., RMSEA, S-RMR, NNFI, CFI) were used to evaluate the five hypothesized models. That is, each fit index provides insight regarding the extent to which data from the Model Development group fit different latent factor models that were hypothesized based on prior research and the introduction of new, reliable, and valid measures of support needs (i.e. the eight P\&A items). The results of these goodness of fit tests are shown in Table 13. 
Table 13

Fit Indices of Support Needs Models

\begin{tabular}{lcccccc}
\hline \multicolumn{1}{c}{ Model } & $\chi^{2}$ & $\mathrm{df}$ & RMSEA & S-RMR & NNFI & CFI \\
\hline 7-factor model & $12,998.57$ & 1,518 & 0.072 & 0.045 & 0.98 & 0.98 \\
7-factor hierarchical model & $13,720.58$ & 1,532 & 0.074 & 0.048 & 0.98 & 0.98 \\
5-factor model (a subset of SIS items) & 3543.26 & 395 & 0.068 & 0.039 & 0.98 & 0.99 \\
5-factor model (all SIS items) & $15,224.84$ & 1,529 & 0.082 & 0.048 & 0.98 & 0.98 \\
Single-factor model & $23,054.25$ & 1,539 & 0.11 & 0.055 & 0.97 & 0.97 \\
\hline
\end{tabular}

Note. $\chi^{2}=$ Chi-Squared value, $\mathrm{df}=$ degree of freedom, RMSEA $=$ Root Mean Square Error of Approximation, S-RMR $=$ Standardized Root Mean Square Residual, NNFI $=$ Non-normed Fit Index, CFI = Comparative Fit Index

Table 13 shows that the first model, the 7-factor model, had acceptable RMSEA (0.072) and good S-RMR (0.045). Its NNFI (0.98) and CFI (0.98) were quite high.

The second model is the 7-factor hierarchical model. In this model, a global Support Needs was a second-order factor, and the seven SIS subscales were first-order factors. Its RMSEA (0.074) was acceptable, and S-RMR (0.048) indicated good fit. The NNFI (0.98) and CFI (0.98) were also quite high, indicating a good fit. Therefore, all indices show that this model fit data from the sample as well as the first model. This model, however, has the advantage of explaining the moderate to high degree of intercorrelation between SIS subscales as shown in Table 12 (which the first model does not).

The 5-factor model using a subset of SIS items yielded slightly more impressive RMSEA (0.068) and S-RMR (0.039) results than the other models. Its NNFI (0.98) and CFI (0.99) indices indicated good fit. It is important to note, however, that using only 30 items does not provide a reasonable comparison to the other models that include all 57 SIS items. Since all 57 items are valid measures of support needs, a structural model that does account for all 57 items is less desirable on a conceptual basis. 
The 5-factor model using all SIS items was tested to determine if the 5-factor structure based on Bossaert et al.'s (2009) could account for additional variables. Although three of the indices were reasonably strong and in the same range as most of the previous models (i.e., the SRMR was below .05, the NNFI and CFI were both 0.98), the RMSEA (0.082) was slightly beyond Browne and Cudeck (1993) guidelines for an acceptable fit.

The single-factor model yielded the poorest fit indicators. Although its S-RMR (0.055) was in acceptable range, the RMSEA (0.11) was far beyond the cut-off point (i.e., 0.08) for acceptability according to Browne and Cudeck (1993). The NNFI (0.97) and CFI (0.97) were acceptable. When comparing all four fit indicators for this model and the other models, this model had the poorest findings for each indicator.

Comparing models. Tables 15 and 16 present Standardized Loadings (SL) and Squared Multiple Correlations $\left(\mathrm{R}^{2}\right)$ of the SIS items of all latent factor models. To judge which latent factor model provides the most suitable structure for data used in this investigation, the fit statistics in Table 13 require further consideration. The most discriminating fit indicator was the RMSEA. In regard to RMSEA, findings in Table 13 suggest that three models (i.e., the two 7factor models and the 5-factor model using a subset of SIS items) reasonably account for CW data and the other two models do not. The three models that yielded acceptable RMSEAs also yielded good S-RMRs, NNFIs, and CFIs, and therefore these three models merit further consideration.

As was discussed previously, the 5-factor model that used only 30 SIS items had a major conceptual shortcoming because it only accounted for a little over half of the items in comparison to the other models under consideration. Certainly, if a model accounting for less items had dramatically better fit indices than alternative models (accounting for more items), then the superior fit could justify overlooking the fact that it explains fewer valid measures of a construct. The 5-factor model with a subset of SIS items, however, was not dramatically better than the two 
7-factor models in regard to its fit indices. Therefore, due to this major shortcoming in

comparison to the other two models, it must be dropped from consideration as the most suitable

latent factor model.

Table 14

Standardized Loadings and Squared Multiple Correlations of SIS Items of a 7-Factor Model, a 7Factor Hierarchical Model, and a Single-Factor Model

\begin{tabular}{|c|c|c|c|c|c|c|}
\hline \multirow{2}{*}{ Item } & \multicolumn{2}{|c|}{$\begin{array}{l}\text { 7-Factor } \\
\text { model }\end{array}$} & \multicolumn{2}{|c|}{$\begin{array}{l}\text { 7-Factor } \\
\text { hierarchical model }\end{array}$} & \multicolumn{2}{|c|}{$\begin{array}{l}\text { Single-factor } \\
\text { model }\end{array}$} \\
\hline & SL & $\mathrm{R}^{2}$ & SL & $\mathrm{R}^{2}$ & SL & $\mathrm{R}^{2}$ \\
\hline \multicolumn{7}{|l|}{ Home Living Activities } \\
\hline HL1 Using the toilet & 0.82 & 0.67 & 0.82 & 0.68 & 0.71 & 0.50 \\
\hline HL2 Taking care of clothes & 0.82 & 0.68 & 0.82 & 0.68 & 0.71 & 0.50 \\
\hline HL3 Preparing food & 0.72 & 0.52 & 0.72 & 0.51 & 0.65 & 0.42 \\
\hline HL4 Eating food & 0.77 & 0.59 & 0.76 & 0.58 & 0.68 & 0.46 \\
\hline HL5 Housekeeping and cleaning & 0.77 & 0.60 & 0.77 & 0.60 & 0.66 & 0.44 \\
\hline HL6 Dressing & 0.85 & 0.73 & 0.86 & 0.73 & 0.74 & 0.54 \\
\hline $\begin{array}{l}\text { HL7 Bathing and taking care of personal } \\
\text { hygiene and grooming needs }\end{array}$ & 0.84 & 0.71 & 0.84 & 0.71 & 0.73 & 0.53 \\
\hline HL8 Operating home appliances & 0.81 & 0.65 & 0.80 & 0.65 & 0.75 & 0.57 \\
\hline \multicolumn{7}{|l|}{ Community Living Activities } \\
\hline $\begin{array}{l}\text { CL1 Getting from place to place throughout } \\
\text { the community }\end{array}$ & 0.61 & 0.37 & 0.61 & 0.37 & 0.54 & 0.29 \\
\hline $\begin{array}{l}\text { CL2 Participating in recreation/leisure } \\
\text { activities in the community settings }\end{array}$ & 0.83 & 0.69 & 0.82 & 0.68 & 0.74 & 0.55 \\
\hline CL3 Using public services in the community & 0.65 & 0.43 & 0.66 & 0.44 & 0.63 & 0.39 \\
\hline CL4 Going to visit friends and family & 0.65 & 0.42 & 0.65 & 0.43 & 0.59 & 0.34 \\
\hline $\begin{array}{l}\text { CL5 Participating in preferred community } \\
\text { activities (church, volunteer, etc.) }\end{array}$ & 0.82 & 0.67 & 0.81 & 0.66 & 0.74 & 0.54 \\
\hline $\begin{array}{l}\text { CL6 Shopping and purchasing goods and } \\
\text { services }\end{array}$ & 0.69 & 0.47 & 0.70 & 0.48 & 0.66 & 0.44 \\
\hline CL7 Interacting with community members & 0.78 & 0.61 & 0.77 & 0.60 & 0.74 & 0.55 \\
\hline CL8 Accessing public buildings and settings & 0.84 & 0.71 & 0.84 & 0.70 & 0.78 & 0.62 \\
\hline
\end{tabular}


Table 14

Standardized Loadings and Squared Multiple Correlations of SIS Items of a 7-Factor Model, a 7Factor Hierarchical Model, and a Single-Factor Model (continued)

\begin{tabular}{|c|c|c|c|c|c|c|}
\hline \multirow{2}{*}{ Item } & \multicolumn{2}{|c|}{$\begin{array}{l}\text { 7-Factor } \\
\text { model }\end{array}$} & \multicolumn{2}{|c|}{$\begin{array}{c}\text { 7-Factor } \\
\text { hierarchical model }\end{array}$} & \multicolumn{2}{|c|}{$\begin{array}{l}\text { Single-factor } \\
\text { model }\end{array}$} \\
\hline & SL & $\mathrm{R}^{2}$ & SL & $\mathrm{R}^{2}$ & SL & $\mathrm{R}^{2}$ \\
\hline \multicolumn{7}{|l|}{ Lifelong Learning Activities } \\
\hline $\begin{array}{l}\text { LL1 Interacting with others in learning } \\
\text { activities }\end{array}$ & 0.71 & 0.50 & 0.71 & 0.51 & 0.69 & 0.48 \\
\hline $\begin{array}{l}\text { LL2 Participating in training/educational } \\
\text { decisions }\end{array}$ & 0.64 & 0.41 & 0.64 & 0.41 & 0.59 & 0.35 \\
\hline $\begin{array}{l}\text { LL3 Learning and using problem-solving } \\
\text { strategies }\end{array}$ & 0.81 & 0.66 & 0.81 & 0.66 & 0.77 & 0.59 \\
\hline LL4 Using technology for learning & 0.60 & 0.36 & 0.60 & 0.36 & 0.56 & 0.31 \\
\hline LL5 Accessing training/educational settings & 0.58 & 0.34 & 0.58 & 0.34 & 0.56 & 0.31 \\
\hline $\begin{array}{l}\text { LL6 Learning functional academics (reading } \\
\text { signs, counting changes, etc.) }\end{array}$ & 0.75 & 0.56 & 0.75 & 0.56 & 0.69 & 0.47 \\
\hline $\begin{array}{l}\text { LL7 Learning health and physical education } \\
\text { skills }\end{array}$ & 0.74 & 0.55 & 0.74 & 0.55 & 0.69 & 0.48 \\
\hline LL8 Learning self-determination skills & 0.81 & 0.65 & 0.81 & 0.65 & 0.75 & 0.57 \\
\hline LL9 Learning self-management strategies & 0.79 & 0.63 & 0.79 & 0.63 & 0.75 & 0.56 \\
\hline \multicolumn{7}{|l|}{ Employment Activities } \\
\hline $\begin{array}{l}\text { EMP1 Accessing/receiving job/task } \\
\text { accommodations }\end{array}$ & 0.67 & 0.45 & 0.67 & 0.45 & 0.66 & 0.44 \\
\hline EMP2 Learning and using specific job skills & 0.84 & 0.70 & 0.84 & 0.70 & 0.73 & 0.54 \\
\hline EMP3 Interacting with co-workers & 0.83 & 0.68 & 0.83 & 0.69 & 0.77 & 0.59 \\
\hline EMP4 Interacting with supervisors/coaches & 0.84 & 0.70 & 0.84 & 0.70 & 0.76 & 0.58 \\
\hline $\begin{array}{l}\text { EMP5 Completing work-related tasks with } \\
\text { acceptable speed }\end{array}$ & 0.85 & 0.73 & 0.85 & 0.73 & 0.73 & 0.54 \\
\hline $\begin{array}{l}\text { EMP6 Completing work-related tasks with } \\
\text { acceptable quality }\end{array}$ & 0.88 & 0.77 & 0.87 & 0.76 & 0.76 & 0.57 \\
\hline EMP7 Changing job assignments & 0.74 & 0.55 & 0.74 & 0.55 & 0.67 & 0.45 \\
\hline $\begin{array}{l}\text { EMP8 Seeking information and assistance from } \\
\text { an employer }\end{array}$ & 0.64 & 0.41 & 0.65 & 0.42 & 0.65 & 0.42 \\
\hline \multicolumn{7}{|l|}{ Health and Safety Activities } \\
\hline H\&S1 Taking medications & 0.71 & 0.51 & 0.71 & 0.50 & 0.67 & 0.45 \\
\hline H\&S2 Avoiding health and safety hazards & 0.85 & 0.72 & 0.85 & 0.72 & 0.82 & 0.68 \\
\hline
\end{tabular}


Table 14

Standardized Loadings and Squared Multiple Correlations of SIS Items of a 7-Factor Model, a 7Factor Hierarchical Model, and a Single-Factor Model (continued)

\begin{tabular}{|c|c|c|c|c|c|c|}
\hline \multirow[t]{2}{*}{ Item } & \multicolumn{2}{|c|}{$\begin{array}{l}\text { 7-Factor } \\
\text { model }\end{array}$} & \multicolumn{2}{|c|}{$\begin{array}{c}\text { 7-Factor } \\
\text { hierarchical model }\end{array}$} & \multicolumn{2}{|c|}{$\begin{array}{l}\text { Single-factor } \\
\text { model }\end{array}$} \\
\hline & SL & $\mathrm{R}^{2}$ & SL & $\mathrm{R}^{2}$ & $\overline{\mathrm{SL}}$ & $\mathrm{R}^{2}$ \\
\hline H\&S3 Obtaining health care services & 0.66 & 0.44 & 0.67 & 0.44 & 0.64 & 0.41 \\
\hline H\&S4 Ambulating and moving about & 0.60 & 0.37 & 0.59 & 0.34 & 0.58 & 0.33 \\
\hline $\begin{array}{l}\text { H\&S5 Learning how to access emergency } \\
\text { services }\end{array}$ & 0.76 & 0.58 & 0.76 & 0.58 & 0.74 & 0.55 \\
\hline H\&S6 Maintaining a nutritious diet & 0.74 & 0.54 & 0.74 & 0.55 & 0.71 & 0.50 \\
\hline H\&S7 Maintaining physical health and fitness & 0.70 & 0.49 & 0.70 & 0.49 & 0.67 & 0.45 \\
\hline H\&S8 Maintaining emotional well-being & 0.60 & 0.36 & 0.61 & 0.38 & 0.60 & 0.36 \\
\hline \multicolumn{7}{|l|}{ Social Activities } \\
\hline SOC1 Socializing within the household & 0.74 & 0.55 & 0.75 & 0.56 & 0.67 & 0.44 \\
\hline $\begin{array}{l}\text { SOC2 Participating in recreation/leisure } \\
\text { activities with others }\end{array}$ & 0.83 & 0.69 & 0.83 & 0.69 & 0.77 & 0.59 \\
\hline SOC3 Socializing outside the household & 0.85 & 0.73 & 0.85 & 0.72 & 0.76 & 0.58 \\
\hline SOC4 Making and keeping friends & 0.82 & 0.68 & 0.82 & 0.68 & 0.75 & 0.57 \\
\hline $\begin{array}{l}\text { SOC5 Communicating with others about } \\
\text { personal needs }\end{array}$ & 0.77 & 0.59 & 0.78 & 0.60 & 0.77 & 0.60 \\
\hline SOC6 Using appropriate social skills & 0.79 & 0.63 & 0.79 & 0.63 & 0.73 & 0.53 \\
\hline $\begin{array}{l}\text { SOC7 Engaging in loving and intimate } \\
\text { relationships }\end{array}$ & 0.67 & 0.45 & 0.67 & 0.44 & 0.63 & 0.39 \\
\hline SOC8 Engaging in volunteer work & 0.67 & 0.44 & 0.66 & 0.43 & 0.65 & 0.43 \\
\hline \multicolumn{7}{|l|}{ Protection \& Advocacy Activities } \\
\hline P\&A1 Advocating for self & 0.83 & 0.69 & 0.83 & 0.69 & 0.78 & 0.61 \\
\hline P\&A2 Managing money and personal finances & 0.57 & 0.33 & 0.58 & 0.33 & 0.52 & 0.27 \\
\hline P\&A3 Protecting self from exploitation & 0.79 & 0.63 & 0.79 & 0.63 & 0.73 & 0.53 \\
\hline P\&A4 Exercising legal responsibilities & 0.80 & 0.64 & 0.80 & 0.64 & 0.75 & 0.56 \\
\hline $\begin{array}{l}\text { P\&A5 Belonging to and participating in self- } \\
\text { advocacy/support organizations }\end{array}$ & 0.72 & 0.51 & 0.72 & 0.52 & 0.68 & 0.47 \\
\hline P\&A6 Obtaining legal services & 0.44 & 0.19 & 0.44 & 0.19 & 0.38 & 0.14 \\
\hline P\&A7 Making choices and decisions & 0.85 & 0.73 & 0.85 & 0.72 & 0.78 & 0.61 \\
\hline P\&A8 Advocating for others & 0.72 & 0.52 & 0.72 & 0.52 & 0.68 & 0.46 \\
\hline
\end{tabular}

Note. $\mathrm{SL}=$ Standardized Loading, $\mathrm{R}^{2}=$ Squared Multiple Correlations 
Table 15

Standardized Loadings and Squared Multiple Correlations of SIS Items of a 5-Factor Model

(Using a Subset of SIS Items) and a 5-Factor Model (Using All SIS Items)

\begin{tabular}{|c|c|c|c|c|}
\hline \multirow[t]{2}{*}{ Item } & \multicolumn{2}{|c|}{$\begin{array}{c}\text { 5-Factor model } \\
\text { (a subset of items) }\end{array}$} & \multicolumn{2}{|c|}{$\begin{array}{c}\text { 5-Factor model } \\
\text { (all items) }\end{array}$} \\
\hline & SL & $\mathrm{R}^{2}$ & SL & $\mathrm{R}^{2}$ \\
\hline \multicolumn{5}{|l|}{ Daily Living } \\
\hline HL1 Using the toilet & 0.85 & 0.72 & 0.82 & 0.67 \\
\hline HL2 Taking care of clothes & - & - & 0.82 & 0.68 \\
\hline HL3 Preparing food & - & - & 0.72 & 0.51 \\
\hline HL4 Eating food & 0.78 & 0.62 & 0.76 & 0.58 \\
\hline HL5 Housekeeping and cleaning & - & - & 0.77 & 0.60 \\
\hline HL6 Dressing & 0.88 & 0.77 & 0.86 & 0.73 \\
\hline $\begin{array}{l}\text { HL7 Bathing and taking care of personal hygiene and } \\
\text { grooming needs }\end{array}$ & 0.84 & 0.71 & 0.84 & 0.71 \\
\hline HL8 Operating home appliances & - & - & 0.80 & 0.65 \\
\hline \multicolumn{5}{|l|}{ Community Living } \\
\hline CL1 Getting from place to place throughout the community & 0.62 & 0.38 & 0.61 & 0.37 \\
\hline $\begin{array}{l}\text { CL2 Participating in recreation/leisure activities in the } \\
\text { community settings }\end{array}$ & 0.83 & 0.70 & 0.82 & 0.68 \\
\hline CL3 Using public services in the community & 0.66 & 0.43 & 0.66 & 0.44 \\
\hline CL4 Going to visit friends and family & 0.66 & 0.43 & 0.65 & 0.43 \\
\hline $\begin{array}{l}\text { CL5 Participating in preferred community activities (church, } \\
\text { volunteer, etc.) }\end{array}$ & 0.83 & 0.68 & 0.81 & 0.66 \\
\hline CL6 Shopping and purchasing goods and services & 0.68 & 0.47 & 0.69 & 0.48 \\
\hline CL7 Interacting with community members & - & - & 0.77 & 0.60 \\
\hline CL8 Accessing public buildings and settings & 0.84 & 0.70 & 0.84 & 0.71 \\
\hline \multicolumn{5}{|l|}{ Personal \& Social } \\
\hline LL1 Interacting with others in learning activities & - & - & 0.70 & 0.49 \\
\hline LL2 Participating in training/educational decisions & - & - & 0.61 & 0.37 \\
\hline LL3 Learning and using problem-solving strategies & - & - & 0.78 & 0.61 \\
\hline LL4 Using technology for learning & - & - & 0.56 & 0.31 \\
\hline LL5 Accessing training/educational settings & - & - & 0.57 & 0.32 \\
\hline $\begin{array}{l}\text { LL6 Learning functional academics (reading signs, counting } \\
\text { changes, etc.) }\end{array}$ & - & - & 0.69 & 0.48 \\
\hline LL7 Learning health and physical education skills & - & - & 0.70 & 0.49 \\
\hline
\end{tabular}


Table 15

Standardized Loadings and Squared Multiple Correlations of SIS Items of a 5-Factor Model

(Using a Subset of SIS Items) and a 5-Factor Model (Using All SIS Items; continued)

\begin{tabular}{|c|c|c|c|c|}
\hline \multirow[t]{2}{*}{ Item } & \multicolumn{2}{|c|}{$\begin{array}{l}\text { 5-Factor model } \\
\text { (a subset of items) }\end{array}$} & \multicolumn{2}{|c|}{$\begin{array}{c}\text { 5-Factor model } \\
\text { (all items) }\end{array}$} \\
\hline & SL & $\mathrm{R}^{2}$ & $\overline{\mathrm{SL}}$ & $\mathrm{R}^{2}$ \\
\hline LL8 Learning self-determination skills & 0.78 & 0.61 & 0.77 & 0.60 \\
\hline LL9 Learning self-management strategies & 0.77 & 0.59 & 0.76 & 0.58 \\
\hline H\&S1 Taking medications & - & - & 0.66 & 0.44 \\
\hline H\&S2 Avoiding health and safety hazards & - & - & 0.83 & 0.68 \\
\hline H\&S3 Obtaining health care services & - & - & 0.64 & 0.41 \\
\hline H\&S4 Ambulating and moving about & - & - & 0.54 & 0.29 \\
\hline H\&S5 Learning how to access emergency services & - & - & 0.74 & 0.55 \\
\hline H\&S6 Maintaining a nutritious diet & - & - & 0.71 & 0.50 \\
\hline H\&S7 Maintaining physical health and fitness & - & - & 0.66 & 0.44 \\
\hline H\&S8 Maintaining emotional well-being & 0.66 & 0.43 & 0.62 & 0.39 \\
\hline SOC1 Socializing within the household & 0.72 & 0.52 & 0.69 & 0.47 \\
\hline SOC2 Participating in recreation/leisure activities with others & - & - & 0.78 & 0.61 \\
\hline SOC3 Socializing outside the household & - & - & 0.78 & 0.60 \\
\hline SOC4 Making and keeping friends & 0.78 & 0.61 & 0.77 & 0.59 \\
\hline SOC5 Communicating with others about personal needs & 0.79 & 0.63 & 0.78 & 0.61 \\
\hline SOC6 Using appropriate social skills & 0.78 & 0.61 & 0.75 & 0.56 \\
\hline SOC7 Engaging in loving and intimate relationships & 0.66 & 0.44 & 0.64 & 0.41 \\
\hline SOC8 Engaging in volunteer work & - & - & 0.65 & 0.43 \\
\hline \multicolumn{5}{|l|}{ Work } \\
\hline EMP1 Accessing/receiving job/task accommodations & - & - & 0.67 & 0.45 \\
\hline EMP2 Learning and using specific job skills & 0.81 & 0.66 & 0.84 & 0.70 \\
\hline EMP3 Interacting with co-workers & - & - & 0.83 & 0.68 \\
\hline EMP4 Interacting with supervisors/coaches & - & - & 0.84 & 0.70 \\
\hline EMP5 Completing work-related tasks with acceptable speed & 0.93 & 0.86 & 0.85 & 0.73 \\
\hline EMP6 Completing work-related tasks with acceptable quality & 0.94 & 0.89 & 0.88 & 0.77 \\
\hline EMP7 Changing job assignments & - & - & 0.74 & 0.55 \\
\hline EMP8 Seeking information and assistance from an employer & - & - & 0.64 & 0.41 \\
\hline
\end{tabular}


Table 15

Standardized Loadings and Squared Multiple Correlations of SIS Items of a 5-Factor Model (Using a Subset of SIS Items) and a 5-Factor Model (Using All SIS Items; continued)

\begin{tabular}{lcccc}
\hline \multicolumn{1}{c}{ Item } & \multicolumn{2}{c}{$\begin{array}{c}\text { 5-Factor model } \\
\text { (a subset of items) }\end{array}$} & \multicolumn{2}{c}{$\begin{array}{c}\text { 5-Factor model } \\
\text { (all items) }\end{array}$} \\
\cline { 2 - 6 } & $\mathrm{SL}$ & $\mathrm{R}^{2}$ & $\mathrm{SL}$ & $\mathrm{R}^{2}$ \\
\hline Protection \& Advocacy & 0.84 & 0.70 & 0.83 & 0.70 \\
P\&A1 Advocating for self & 0.57 & 0.32 & 0.57 & 0.33 \\
P\&A2 Managing money and personal finances & 0.79 & 0.63 & 0.79 & 0.63 \\
P\&A3 Protecting self from exploitation & 0.80 & 0.64 & 0.80 & 0.63 \\
P\&A4 Exercising legal responsibilities & 0.71 & 0.50 & 0.72 & 0.51 \\
P\&A5 Belonging to and participating in self-advocacy/ & & & & \\
$\quad$ support organizations & 0.43 & 0.19 & 0.44 & 0.19 \\
P\&A6 Obtaining legal services & 0.86 & 0.73 & 0.85 & 0.73 \\
P\&A7 Making choices and decisions & 0.72 & 0.52 & 0.72 & 0.52 \\
P\&A8 Advocating for others & & & & \\
\hline
\end{tabular}

Note. $\mathrm{SL}=$ Standardized Loading, $\mathrm{R}^{2}=$ Squared Multiple Correlations

In considering the suitability of the two 7-factor models, it is noteworthy that the hierarchical model explained the intercorrelation between the subscales (see Table 12) and the nonhierarchical model does not. Therefore, the 7-factor hierarchical model is a more logically coherent model and provides a more parsimonious explanation of data. Because the 7 -factor hierarchical model was determined to be the most suitable model (i.e., the model of best fit), data from the other half of the data set, the Model Testing sample, were used to test its parameters.

Model validation. In validating the 7-factor hierarchical model, LISREL 8.8 (Jöreskog \& Sörbom, 2006) was run on the Model Testing group (1,961 CW participants) with Support Needs as the second-order factor and the seven subscales as first-order factors. Intercorrelations among the 57 SIS items in the Model Testing group ranged from 0.75 to 0.94 which are very close to the Model Development group (0.73 to 0.91). Its RMSEA (0.077) again showed an acceptable fit, and S-RMR (0.047) was good. Its NNFI (0.98) and CFI (0.98) were also in good 
range. These fit indices were nearly identical to those of the Model Development group. Therefore, the 7-factor hierarchical model was validated.

\section{Chapter Summary}

Cluster analysis procedures and factor analysis procedures were applied to data measuring the support needs of 3,998 adults with ID/DD. Findings from cluster analyses provided support for 5 clusters (groups) of people based on intensity of support needs. Findings from factor analyses provided support for a 7-factor hierarchical latent factor structure of the support needs construct. Limitations of this study, implications of these findings for public policy and professional practice in the field of ID/DD, and implications for future research will be discussed in the next chapter. 


\section{CHAPTER V}

\section{CONCLUSION: SUMMARY AND RECOMMENDATIONS}

For several decades, a variety of scholars in the fields of disability studies and special education have pointed out that all disability conditions, including intellectual disability (ID), are social constructions. The invention (i.e., social construction) of a disability condition occurs when (a) differences among people are observed and documented, (b) it is noticed that some people share the same, or similar, types of differences, (c) these differences are given a name, and (d) the name becomes part of the professional nomenclature (e.g., see Kollock \& O'Brien, 1994; Mercer, 1973; Sleeter, 1986). Thompson and Ezell (2005) pointed out that just because a disability is socially constructed does not mean that it is somehow not real as some have suggested (e.g., Danforth \& Rhodes, 1997; Ysseldyke et al., 2000). Just because something is real, however, does not mean it is important. If the differences associated with a disability condition are largely superficial and do not impact people's lives or functioning, there is little purpose served by trying to understand such a disability condition at a deeper level. For example, people with 20/30 vision do not have perfectly crisp vision, but their deficits in visual acuity are not sufficiently unusual nor do they significantly limit life activities. Therefore, because the ramifications of 20/30 vision are limited, it would not make sense to invest a great deal of time and effort into investigating the life experiences and conditions of this particular population.

The important issue is not whether a disability condition is a social construction (all disability conditions are), but the extent to which a disability condition actually impacts the lives of people who meet criterion for diagnosis. People with intellectual and related developmental disabilities (ID/DD) have long been understood as a population with a disability that does 
significantly impact life experiences and daily functioning. ID has also traditionally been understood as a deficit condition, evidenced by limitations in skill acquisition. The lack of skill acquisition affects people's personal competence. This "deficit-based" understanding of ID has resulted in using medical and behavioral interventions to address the causes of ID and its impact.

More recently, however, there has been an increased awareness that understanding people by their deficits can yield diminishing returns. In most cases of ID (and related developmental disabilities), the deficits are not fully fixable. Therefore, despite the best efforts, people with ID/DD remain in a state of relative incompetence when it comes to engaging in valued settings and life activities. Requiring people with ID/DD to achieve a readiness level of skills prior to participating in certain life activities and valued settings severely restricts opportunities to enjoy a high quality of life. That is, because most people with ID/DD never become sufficiently competent (i.e., ready) to navigate environments independently and participate fully in experiences that general population values, they miss out on many meaningful life activities and are forced to live much of their lives separated from the rest of society.

A broader understanding of ID/DD calls for considering people in the context of how their deficits and limitations interact with the demands and expectations of community environments. This social-ecological orientation to understanding ID/DD focuses attention away from fixing deficits and limitations and toward identifying and arranging supports needed to function in everyday environments and meaningfully participate in culturally valued life activities. The past decade, in particular, has witnessed considerable interest in a socialecological understanding of ID/DD. The findings reported in this dissertation contribute to the research literature and professional efforts that are focused on understanding people with ID/DD by their support needs. 


\section{Summary of Procedures, Findings, and Discussions}

Two studies were conducted which involved analyzing data from a large, statewide sample of people with ID/DD. This data set included demographic information, information related to living arrangements or residential integration as well as community risk, and data from the Supports Intensity Scale (SIS). The SIS is an assessment scale that measures the support needs of people with ID/DD, and is widely used throughout North America as well as select European and Asian countries (Nygren, 2011).

The first study applied cluster analysis procedures to identify how people with ID/DD might be classified according to their support needs. This study contributed to the professional literature in several ways. First, up to this point in time, the only approaches to support needs classification that had been proposed were based on conceptual work. That is, there was no empirical basis for classifying people into support needs categories. Second, there has actually been very little published research on the application of cluster analysis to special education or disability populations. Thus, this study was at the forefront of utilizing this statistical tool to further knowledge in special education and disability related fields. And finally, this study incorporated data from the SIS Supplemental Protection \& Advocacy (P\&A) Scale in the analyses. Only recently have researchers learned that the P\&A scale provides valid and reliable measures of support needs, and this study was one of the first to analyze P\&A data alongside data from the six subscales (i.e., Home Living, Community Living, Lifelong Learning, Employment, Health \& Safety, and Social Activities) that make up the standardized portion of the SIS.

The second study used factor analysis procedures to expand a line of research examining the factor structure of the SIS and the support needs construct (which the SIS purports to measure). As with the cluster analysis study, this study made several unique contributions to the professional literature. First, few prior studies used confirmatory factor analytic (CFA) techniques to test the fit (i.e., alignment with data) of competing latent factor models. Reviewing 
professional literature and identifying prior models with empirical support eliminated the need to conduct exploratory factor analysis to arrive at a hypothesized model, and this is the first study to test all of the competing models proposed from prior research. Second, the current study used a comprehensive data set that included a representative sample of people with ID/DD on which to evaluate structural models; several of the prior studies in this line of research were based on data collected on a sample with questionable representativeness (e.g., Harries et al. [2005] used an institutional population, Bossaert et al. [2009] used a population with disabilities other than ID/DD). Third, as with the clustering study, data from the P\&A scale were included in the analyses. Thus, an expanded pool of support needs measures was included in this study that had not been included in prior investigations. Fourth, the large size of the data set was unique, and it provided the opportunity to split the sample into both a Model Development group and a Model Testing group. Using half of the sample to compare competing models against fit statistics, and then examining the extent to which the model of best fit was validated from data from the other half of the sample provided a uniquely comprehensive and rigorous examination of the factor structure of support needs.

\section{Findings and Implications Related to Research Question 1}

Research question 1 was "What multiple grouping model, based on differing support needs, is generated from a statewide sample of people with ID/DD using cluster analysis?" Four grouping models were evaluated. The most conceptually and statistically robust models was the 5-cluster solution. The model had the most clusters with significantly different centroids. Other personal information (i.e., community safety risk, CW funding level, type of living arrangement, use of individual employment, use of community services, use of habilitation, use of supported employment, and use of sheltered employment) of CW participants also significantly contributed to the cluster membership. Nevertheless, variables associated with language, gender, and age did not. 
Characteristics and needs of members in each cluster. The 5 clusters are best

understood by differing intensities of support needs. Cluster 1 had the lowest intensity of support needs, followed by Clusters 2, 3, 4, and 5 respectively. Both subscale scores and the composite score (i.e., the SNI score) supported the classification by intensity framework. That is, people with the most intense Home Living support needs generally had the most intense Social support needs, Employment support needs, and so forth, as well as the highest SNI scores.

These findings support the conceptual work of Luckasson et al. (1992) who argued that people with ID/DD could be classified by intensity of support needed (although they called for a 4-tier classification system and not a 5-tier one). Also, the findings in this study are consistent with those reported by Wehmeyer et al. (2012) who compared support needs of 274 adults with ID and other disabilities. Their data showed that the participants who had more intense overall support needs as reflected in SNI scores tended to have more intense support needs across all SIS subscales.

In one sense, it would have been more interesting to have discovered an array of clusters with people with different intensities of support needs in different areas of life. Perhaps one cluster would include people needing intense support at home but not needing intense support on a job or in social situations, while another cluster would be made up of people who needed very intense support in a job and in other community situations, but little support in their homes. If such findings would have been forthcoming, it would certainly support the division of a service delivery system along different domains of life (e.g., a service system for employment, a service system for residential). Data from this investigation, however, simply did not support such findings.

The critical finding from the current investigation was that there was an empirical basis for grouping people by relative intensity of support needed. In conjunction with findings from the second investigation, this finding suggests a structural model of support needs where a global, 
latent factor underlies support needs for different domains of life. Therefore, it suggests that planning teams should plan for supports across life domains (i.e., support needs in one domain will likely be relevant to other domains in terms of intensity). Future research may show that supports may take different forms in different domains; however, the intensity of support needs is most likely consistent across domains for most people. Because, as a general rule, the intensity of support needs across domains will likely be similar, people with ID/DD can best be understood as falling along a continuum of intensity of needed supports.

The clusters, however, are not equally populated. This finding has important implications for how service systems are organized and supports are delivered. As can be seen in Figure 2, over half (53\%) of people in the sample fell into support-intensity Clusters 4 and 5 (the two clusters with people with the relatively most intense support needs). In contrast, Clusters 1 and 2 (people with the relatively least intense support needs) made up only $25 \%$ of the sample. The service delivery system needs to take particular note of the sizable minority in Clusters 1 and 2.

Figure 2. Percentages of People with ID/DD $(\mathrm{n}=3,998)$ in Five Support-Intensity Clusters

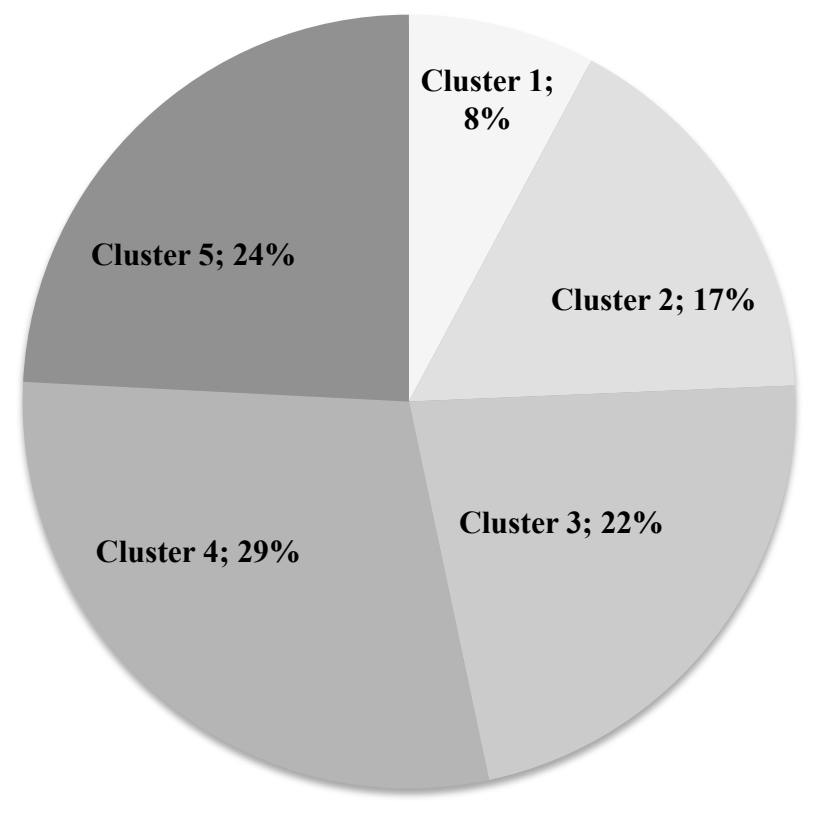


There is a long history of concerns regarding the tendency to over-protect people with ID/DD (Perske, 1972), and there is considerable evidence that people with ID/DD with relative less intense support needs consider the services funded by governmental units to be overly intrusive (see Edgerton \& Bercovici, 1976; Tymchuk, Lakin, \& Luckasson, 2001). Moreover, supporting people in overly intrusive ways wastes precious public resources and, therefore, exacerbates the problem of limited access to services as evidenced by the extensive waiting lists for services in the U.S. (Braddock et al., 2013).

Naming the clusters. In its $9^{\text {th }}$ edition of the Definition and Classification Manual, the AAIDD proposed the ILEP classification system. This system classified people with ID/DD by supports intensity into four groups based on judgments regarding a person's need for supports (Luckasson et al., 1992). The names of the four support needs classification groups were Intermittent, Limited, Extensive, and Pervasive. Although findings from this study suggested five clusters of people with different intensities of support needs, the approach to classifying people by relative intensity of support needs was validated.

AAIDD's approach to naming the clusters, however, should be thoughtfully considered in light of past as well as recent history with classification terms. The first consideration is the history of clinical terms becoming pejorative terms (e.g., moron, imbecile, idiot). This possibility would appear to be less likely due to the nature of AAIDD's terminology (i.e., Intermittent, Limited, Extensive, Pervasive). These terms clearly refer to relative magnitude and are not the types of ambiguous nouns that do not suggest a point of comparison. Ambiguous worded nouns (e.g., educable, imbecile) that have no point of comparison would seem to be more susceptible to acquiring baggage because their meaning is so open to interpretation.

Nevertheless, terms that suggest a point of comparison or measurement also have a checkered history. Classification terms can lead to low expectations (Beirne-Smith, Ittenbach, \& Patton, 2006), and there is also danger that the "least extreme" term becomes understood as 
representing inconsequential phenomena (Snell et al., 2009). This particular problem has most recently occurred with the classification category of "Mild ID." People diagnosed in this category are a vulnerable population for many reasons, not the least of which is that their relative strengths can mask important vulnerabilities. There are many people with Mild ID who have good verbal, motor, and functional skills but are quite gullible and vulnerable to manipulation by others (Greenspan et al., 2001). Also, many with Mild ID have great difficulty developing sufficient literacy and mathematical skills to be competitive in modern jobs or meet the expectations of academic and vocational programs in post-secondary educational settings. Therefore, Mild ID is not simply a mild challenge that is quickly or easily overcome.

Rather, people with Mild ID need ongoing supports to attain desired personal outcomes and experience a high quality of life (Tymchuk et al., 2001). Snell et al. (2009) discussed how misleading Mild ID can be if it is interpreted as needing mild supports. Although people with ID with relatively higher IQ scores generally need different types of supports than people with ID/DD with relatively lower IQ scores, different does not necessarily mean less intense in all aspects of life. Moreover, different support needs certainly does not mean inconsequential support needs.

Therefore, in naming the five clusters in this study, the wisest course of action is to resist terminology that provides openings for misinterpretation down the line, such as Intermittent, Limited, Extensive, and Pervasive. It would be better to simply stick with numbers that reflect the findings of this investigation. Namely, people with ID/DD can be grouped on the basis of having relatively similar intensities of support needs and identifying five groups provides the best way to distinguish one group from another. Therefore, Support Needs Level 1, Support Needs Level 2, and so on will be used to name the five groups.

Before proceeding further, there is one consideration that cannot be overemphasized. Namely, the supports needed by each individual within a cluster are going to be uniquely 
personal and must be based on a variety of considerations. Because supports must be identified and arranged to meet individual needs and circumstances, there can be absolutely no definitive "recipe" of supports to meet the needs of all members of any particular cluster.

Names and concise descriptions of the five clusters are:

- Support Needs Level 1 - People with the relatively lowest intensity of support needs. Members of this group receive relatively low levels of resource allocation from the government; nearly $80 \%$ were allocated the lowest of six funding tiers (compared to $27 \%$ of the total sample at this level). They are also more likely than others to use individual and supported employment services, and live in the least restrictive community residences (either host home or other Individual Residential Services and Supports that have a small number of housemates such as foster care homes without the formal foster case relationship). Very few members of this group were judged to pose a community risk.

- Support Needs Level 2 - People with slightly more intense support needs than those in Support Needs Level 1, but less intense support needs than $75 \%$ of people with ID/DD. These participants were also more likely to work on community jobs (individual and supported employment) than members of other clusters (except Support Needs Level 1). Although not particularly common, Support Needs Level 2 members were more likely to be considered a community safety risk due to a history of involvement with the criminal justice system $(4 \%$ had a prior conviction) than people in other groups. The amount of funding from the state was more varied than Support Needs Level 1 members. Nearly 75\% of Support Needs Level 2 members, however, were in the lowest or second to lowest funding tier (out of a possible six tiers).

- Support Needs Level 3 - People with support needs measured in the mid-range. Nearly $11 \%$ were judged to pose a community risk, a percentage that is almost twice as high as any other clusters. The funding amount received by this group were relatively evenly distributed between levels of less funding (i.e., Level 1), mid-ranged funding (i.e., Levels 2 and 3), and more 
funding (i.e., Levels 4, 5, and 6). Their residential living arrangements were more diverse than the first two groups. Sheltered employment was at the highest percentage in this group compared to all other groups.

- Support Needs Level 4 - People with support needs measured at the second highest level. This group was the most populous group, accounting for a full $29 \%$ of people in the sample. Support Needs Level 4 members were likely to use community services and habilitation services, but very few were employed in supported employment and individual employment jobs. The funding amounts received by members of this group were distributed across the six funding levels, and their residential living arrangements were also diverse.

- Support Needs Level 5 - People with the highest SIS scores among all clusters.

Members of this group received the most funding from the state, with $62 \%$ of the members in the highest two funding levels (Support Needs Level 4 members, the second highest funded group, had only $20 \%$ in the top two funding tiers). Support Need Level 5 members were far more likely to live in more restrictive housing with multiple housemates; a full $20 \%$ lived in 8 -bed group homes. Individual and supported employment jobs were uncommon. Compared to the other clusters, members of this group were the most likely to use community services and habilitation services.

Age, language spoken, and gender of $\mathrm{CW}$ participants were variables that did not contribute to the clustering solution. This finding supported findings reported in the SIS Users Manual (Thompson et al., 2004b) and by other researchers (e.g., Kuppens et al., 2010) that the SIS scores do not differ across age and gender groups.

It is important to stress that the 5-factor cluster solution reflects supports and services that are received at the current time, and not supports and services that people necessarily should be receiving. For instance, proponents of supported employment (e.g., see Cimera, 2011; Wehman, Kregel, Banks, Hill, \& Moon, 1987) have long argued that all people with disabilities are 
appropriate candidates for supported employment jobs, and these proponents maintain that all really does mean all. In other words, diagnosis, extent of limitations, or intensity of support needed should not have any bearing on a person's access to supported employment. Therefore, the finding that those in the Support Needs Level 1 Cluster are far more likely to work on supported employment jobs than those in the Support Needs Level 5 Cluster is evidence that those with less intense support needs are being placed into supported employment jobs at a higher rate than those with more intense support needs. This finding, however, does not mean that people in the Support Needs Level 5 Cluster should not be considered as viable candidates for supported employment. Rather, the finding suggests that the Colorado service system is "cherry picking" people with ID/DD whose lower intensities of support needs make them the easiest to place in supported employment jobs. Moreover, the Colorado service system has not been particularly effective in providing supported employment services to those with more complicated needs. Unfortunately, data from Braddock et al. (2013) reveal that Colorado service system is quite typical in regard to supported employment. Not only are supported employment jobs limited, individuals with ID/DD with more complex needs are rarely provided with supported employment opportunities.

Supports need to be identified and arranged based on each person's (a) desired life experiences, preferences, and goals; and (b) individual support needs (Thompson et al., 2009). There should be no confusion about the fact that, although two people within a cluster may share support needs in terms of relative quantitative intensity, these same two people may have qualitatively different support needs based on many different considerations. Most certainly, no two people will want and need to be supported in exactly the same way.

\section{Findings and Implications Related to Research Question 2}

Research question 2 was "Using confirmatory factor analysis, which structural model of support needs is best supported by data from a statewide sample of people with ID/DD?" Five 
structural models were evaluated. Two of the models aligned with the SIS subscale structure (i.e., a 7-factor model and a 7-factor hierarchical model). Additionally, a 5-factor model using a subset of SIS items, a 5-factor model with all SIS items, and a single-factor model were investigated. Each model was based on prior conceptual or empirical work that was published in the professional literature. The 7-Factor Hierarchical Model (Model 2 in Appendix A) was judged to be the most suitable.

A multidimensional, effect-indicator model of support needs. Bollen and Lennox (1991) distinguished structural models comprised of causal indicators from those comprised of effect indicators. Causal indicator models are ones where the observed variables influence (i.e., cause) the first-order latent factors, which in turn influence or cause second-order factor(s). In contrast, with effect indicator models, the second-order factors are assumed to cause the lowerorder factors, which in turn influence the observed variables. The 7-Factor Hierarchical Model of Support Needs that emerged from this investigation is clearly an effect indicator model. The second-order latent factor, which was labeled "Support Needs", represents a global, overarching influence on the intensity of a person's support needs in all areas of life.

In contrast, Thompson, McGrew, Johnson et al. (1999) used factor analytic procedures to validate a 7-factor causal indicator model of community adjustment using post-school follow-up data from former high school students with disabilities. Similar to a model of socioeconomic status (SES; where observed variables such as salaries, employment level, education level all contribute to a global SES factor but a global measure of SES does not cause salaries, employment, education), the latent factors in Thompson, McGrew, Johnson et al.'s Community Adjustment Model were considered to be caused by the post-school outcome measures (i.e., observable data). In other words, the seven latent factors associated with community adjustment were not understood to be the causal agents of the observed variables, but rather the observed variables were considered to be the causal agents of the latent (i.e., first-order) factors. 
Thompson, McGrew, Johnson et al. (1999), however, indicated that there was one dimension (i.e., latent factor) in their community adjustment model that was more suitable for consideration as an effect indicator dimension. They stated that the latent factor they labeled Need for Support Services was "unique from the other dimensions of the model in that the indicators associated with it are effect indicators as opposed to causal indicators" (p. 94).

Because Thompson, McGrew, Johnson et al.'s Community Adjustment Model was instrumental in the early conceptualization of the SIS (personal communication, J. R. Thompson, November 11, 2013), it is noteworthy that the 7-Factor Hierarchical Model of Support Needs emerging from this investigation was consistent with Thompson, McGrew, Johnson et al.'s assertion. Support needs is conceptually an effect indicator latent factor. This consideration is conceptually consistent with defining support needs as "a psychological construct referring to the pattern and intensity of supports necessary for a person to participate in activities linked with normative human functioning" (Schalock et al., 2010, p. 224). Also, it is logically consistent with a global score on a support needs assessment scale, such as the SNI score on the SIS.

Support needs - the second-order factor. According to the structural model shown in Model 2 in Appendix A, people with highly intense overall support needs (i.e., the second-order factor) will likely have highly intense support needs in all of the first-order factors, as well as all of the observed variables. Of course, there is sufficient variability among the first-order factors and sufficient variability among the observed variables to conclude that important information can be gleaned from first-order factors as well as units of measurement. The various ways in which SIS composite score (corresponding to the second-order factor), subscale scores (corresponding to first-order factors), and item scores (corresponding to observable data) have been used at the individual, organizational, and jurisdictional levels to guide planning and decision have recently been discussed by Buntinx and Schalock (2010), Kimmich et al. (2009), Thompson et al. (2004b), and van Loon et al. (2010). 
First-order factors. A close examination of the nature of the seven first-order factors in the 7-Factor Hierarchical Model of Support Needs is warranted. According to the 7-Factor hierarchical model, the first-order factors are highly correlated with one another because they share a common underlying factor (i.e., global support needs - the second-order factor). As mentioned, however, although the seven first-order factors share variance and are related to another, each one also provides unique information. Each factor is aligned with an identified subscale on the SIS and is described below:

- Home Living Support Needs. This factor influences eight items that focus on supports that people with ID/DD need to participate in activities related to life in neighborhood homes located in local communities. Included are items related to activities involving personal care, as well as activities involving upkeep and cleanliness of personal belongings and the home environment. Although the focus is on life activities within the home, there is one item (toileting) that also applies to settings outside of the home.

- Community Living Support Needs. This factor influences eight items that focus on supports that people with ID/DD need to participate in activities in public settings in local communities. Items related to getting around one's community, participating in recreation activities in the community, interacting with community members, shopping for and purchasing goods and services, and using public services are included.

- Lifelong Learning Support Needs. This factor influences nine items that focus on supports that people with ID/DD need to be actively engaged in learning activities that are suitable for adults. Included are items related to supports needed to participate in educational decisions, access educational settings (e.g., community college classrooms), manage learning tasks (e.g., self-determination, self-management, problem-solving, using technology), and interact with others (e.g., teachers, fellow students) in educational settings and learning activities.

- Employment Support Needs. This factor influences eight items that focus on supports 
that people with ID/DD need to obtain and maintain employment in community workplaces. Items include supports necessary to access or receive job accommodations, seek information and assistance from an employer, interact with supervisors and coworkers, and complete work with proper speed and quality.

- Health \& Safety Support Needs. This factor influences eight items that focus on supports that people with ID/DD need to be healthy (e.g., free from disease and pain) and feel safe (e.g., free from a fear of eminent danger). Items focus on daily routines (e.g., taking medications), ongoing wellness activities (e.g., maintaining a nutritious diet, avoiding health and safety hazards), and point-in-time activities (e.g., accessing emergency services, obtaining health care services).

- Social Support Needs. This factor influences eight items that focus on supports that people with ID/DD need to socialize in adult life activities within and outside of the home, and to maintain positive relationship with others. Included are items pertaining to socializing, communicating, and building different types of relationship (e.g., friendships, intimate or romantic relationship). Communication supports are embedded within several items (e.g., communicating with others about personal needs).

- Protection \& Advocacy Support Needs. This factor influences eight items that focus on supports that people with ID/DD need to advocate for themselves and protect their civil rights. Items associated with self-determination (i.e., advocating for self, making choices and decisions, belonging to and participating in self-advocacy/support organizations), legal rights and responsibilities (i.e., exercising legal responsibilities, obtaining legal services), offering assistance to others (i.e., advocating for others), and managing finances and protecting self from manipulation (i.e., managing money and personal finances, protecting self from exploitation) are included. 


\section{Implication of Findings for National Goals}

The findings from this investigation have important implications for professional practice and public policy in the field of ID/DD. These implications will be discussed in the context of six national goals, according to Lakin and Turnbull's (2005) book detailing national goals for people with ID/DD for the next decade. These goals were the result of a multi-year effort to gather input from a wide range of experts in the field (i.e., prominent researchers; state and national officials in public policy, health, social service delivery, and advocacy; leaders of parent and consumer advocacy groups) in order to identify collective goals that could serve to guide work in the field of ID/DD. The culmination of the information-gathering phase was a national conference (i.e., the National Goals Conference) where 250 experts gathered to discuss, refine, and eventually adopt six national goals. Although these goals were developed specifically for the U.S., due to the globalization of the field of ID/DD the goals would certainly be applicable to a wide range of countries and regions throughout the world. Below, each goal is presented along with implications from findings from this study.

\section{Goal 1: Increasing Self-Determination and Personal Control in Decisions Affecting People With ID/DD and Their Families}

This goal is based on the premise that if people with ID/DD (and their families) are better encouraged to become the causal agents in the own lives, the service system will become more responsive to people's needs and personal outcomes will improve. Unfortunately, although selfdetermination is a concept that almost everyone can rally around in the abstract, it is such an ethereal concept that practitioners, family members, and people with ID/DD have struggled with how to put the concept into action (Ward, 2005).

The findings in this investigation certainly do not fully solve this problem, but would appear to be helpful in making progress. Through the Protection and Advocacy Support Needs factor, stakeholders can be confident using a set of items that accurately assess the intensity of 
support people with ID/DD need to make decisions about key aspects of their lives and express their preferences. Additionally, a critical aspect of self-determination is awareness of one's own support needs. A person cannot effectively advocate for supports on a job, accommodations in a post-secondary education setting and so on without understanding what supports are needed. It is important that teachers, service provider staff, and family members also have a strong understanding of an individual's support needs. The classification approach that emerged from this study can contribute to helping people with ID/DD understand their own needs for support, as well as help others understand people by their support needs.

\section{Goal 2: Providing Opportunities to People With ID/DD to Live and Participate in Their Own Communities}

Lakin and Stancliffe (2007) documented the dramatic success of deinstitutionalization in the U.S. over the past several decades. The most dramatic decrease occurred between 1977 and 2002, where the percentage of people with ID who received residential services funded by state governments decreased from $83 \%$ to $16 \%$. Braddock et al. (2013) reported that a decline in institutional populations has continued with corresponding increases in the numbers of people living in communities. Twelve U.S. states no longer operate institutions and the national institutional population is now below 30,000. As this national goal implies, however, physical presence in the community is not the same social participation in the community.

The findings from this investigation are certainly applicable to this national goal. The 7Factor Hierarchical Model of Support Needs reveals a global support needs factor that influences the intensity of a person's support needs in all areas of life, as well as the seven dimensions of support that correspond to specific life areas. The validation of this model suggests that professionals, family members, and people with disabilities can be confident in obtaining a comprehensive profile of a person's support needs when using the SIS to measure support needs. A comprehensive overview of supports needed to participate in community life should greatly 
enhance a planning team's ability to plan supports that result in successful inclusion. Van Loon et al. (2010) provided a detailed case study showing how support needs information can inform decision-making in regard to community participation, and how standardized personal outcomes measures can be used to measure the success of efforts to more fully integrate people with ID/DD in local communities. Future research using outcome data in concert with support needs assessment data could provide a wealth of information about how to better support people to live and participate in their local communities.

Further research on the classification categories identified in this investigation may also prove to be helpful in reaching this national goal. Data from this investigation show that people with more intense support needs (i.e., the three highest Support Needs Levels) had very low levels of participation in individual employment and supported employment jobs. Future research could investigate why individuals in these categories are either not being considered to be good candidates for individual or supported employment, or investigate obstacles (and how to overcome obstacles) to successfully place people with more intense support needs in integrated work settings.

\section{Goal 3: Improving Quality of Life for Individuals and Families as They Define It for}

\section{Themselves}

The broadness of this particular goal is such that several implications from findings from this study (and many other studies) might apply. The real focus of this goal (which is clearly stated in the book of Lakin and Turnbull), however, is on people being able to tell the service delivery system how they want to be supported as opposed to the system defining and limiting the types of supports available. It is a reaction against the "program mentality" and "service silos" that began to characterize community-based services after deinstitutionalization gained momentum, and an industry of community service provider organizations emerged (see Wehmeyer, 2013). The classic example of a program mentality taking hold is a Saturday 
morning bowling program for people with ID/DD (such programs are still very common throughout the U.S.). Although there are people with ID/DD who enjoy going bowling on Saturday mornings, very few were asked what types of recreational or leisure experiences they would prefer or like to try. Many would probably prefer to bowl at sometime other than Saturday morning (e.g., in a bowling league with other adults on Tuesday night), and some might not want to join that activity "every" Saturday (e.g., go bowling every two months). The point is, the community-based service delivery system was usually set up from the top down - a limited number of congregate programs for people with ID/DD were established, and people with ID/DD were more or less told to "take it or leave it." This goal is intended to encourage a more bottomup approach to community supports, where individuals with ID/DD identify what they want to do and how they want to be supported, and the service system is charged with responding to people's requests and needs.

There are many who have argued that best avenue to breaking the program mentality (or service silos culture) of service provider organizations and replacing it with a consumer driven or person-centered approach is to send public funding to people directly instead of directly funding service provider organizations. The reasoning is that if people with ID/DD are provided with individual budgets, they will have decision-making authority over what services and supports they desire for the life activities they prefer (see Moseley, Gettings, \& Cooper, 2003). The SIS has been (and still is) used as a tool to determine individual budgets (Kimmich et al., 2009), and the findings from this investigation certainly support its continued application. With identification of seven domains of support needs (through the factor analytic study), policymakers could investigate the correspondence between support needs in a given domain and funding levels that would be appropriate to meet those needs. Certainly, if the premise is true that people with relatively more intense support needs will usually require additional funding to participate in community life than people with relatively less intense support needs, then classifying people by 
the intensity of their support needs (as was accomplished by the cluster analysis study) would be a reasonable basis on which to establish basal funding levels. Although a detailed analysis of resource allocation issues in the field of ID/DD is beyond the scope of this dissertation, there is widespread concern over perceived inequities and inefficiencies in traditional approaches to funding. Historical expenditure rate schedules, idiosyncratic negotiations with service providers, estimates for service operating costs are just a few of the factors contributing to the need to individualize budgeting in the hopes of creating a more transparent, fair, and responsive system (Kimmich et al., 2009; Stancliffe \& Lakin, 2005). Thoughtful use of support needs assessment data can be part of the solution.

\section{Goal 4: Supporting Families as the Most Important and Permanent Unit of Development, Protection, and Lifelong Assistance to Persons With ID/DD}

The findings from this investigation contribute to a research line focusing on obtaining a better conceptualization of the support needs construct as well as enhancing insight into understanding people with ID/DD on the basis of their support needs. This study's findings have implications for supporting families who, for the vast majority of people with ID/DD, are the most important and permanent source of support. Validating the SIS factor structure, and thus the dimensions of the support needs construct, may prove to be useful when communicating with families. The essence of factor analysis is data reduction, in the sense that the process involves taking a large number of variables and reducing them to factors that explain most of the variance. Families can be overwhelmed by number of questions and units of data involved in collecting SIS information. The results of this study, however, show there is an empirical basis for interpreting scores in the context of seven broad support dimensions, as well as an overall score. This factor structure supported by findings from this investigation provides a means to more clearly explain the results of a SIS assessment. Because families are the most important source of support for people with ID/DD, it is very important for them to understand the person's support needs. 
It is also critical to point out that a SIS assessment is done "with people" not "on people," as is the case with so many traditional assessments in the field of ID/DD. The SIS Users Manual (Thompson et al., 2004b) strongly recommends using family members as respondents when completing the scale. Gathering information from family members when completing the SIS assures that the perspectives of family are considered and engages them in the assessment process, a process that many family members have traditionally viewed as mysterious (Turnbull, Turnbull, Erwin, Soodak, \& Shogren, 2011).

\section{Goal 5: Investing in Each Individual's Developmental Potential and Capacity to Contribute in Age-Related Roles as Productive, Respected Community Members}

As has been mentioned in several sections of this dissertation, a focus on a person's support needs shifts assessment in the field of ID/DD from a descriptive approach of deficits to a proscriptive approach that asks a simple question, "What supports does the person need to be more successful in age-related roles as productive, respected community members?" In that regard, the assessment of support needs is the first step in a transformation to a positive mind set (as opposed to a mind set based on defectology). Although there is certainly a reciprocal relationship between intensity of support needed and degree of deficit, findings from this investigation confirm that measures of deficits are not suitable proxies for measures of support needs. This was evident in the findings from the cluster analysis in regard to ratings of community risk (see Table 10). The Support Need Level 2 and Support Need Level 3 Clusters (i.e., those with relatively low and mid-ranged overall support needs) had the largest percentage of people identified as high community risk, not the Support Need Level 5 Cluster.

The findings from this investigation support the conclusion that the SIS provides a valid means by which to assess individual support needs. The ability to measure key concepts is often related to progress in a field, and the field of ID/DD is no exception. Reliable and valid support needs assessment results provided information that can inform decision-making. Decisions that 
are based on solid information have a much greater likelihood of promoting actions that result in enhanced human performance and personal outcomes, as is called for in this goal.

\section{Goal 6: Assuring Access to Sufficient, High-Quality Health and Social Supports to Protect Each Person's Health, Safety, Rights, and Well-Being}

As was mentioned earlier, research and practice that is focused on developing systematic ways to assess and deliver supports people need are likely to result in many positive outcomes, including "sufficient, high-quality health and social supports to protect each person's health, safety, rights, and well-being." The Health \& Safety Support Needs, Social Support Needs, and Protection \& Advocacy Support Needs dimensions of the 7-Factor Hierarchical Model of Support Needs are directly related to the focus of this goal. A strong internal consistency of data and a strong intercorrelation between the Supplemental Protection \& Advocacy Scale and other six latent factors confirmed the conclusion of Thompson et al. (in press) that this portion of the SIS should be included as a subscale in the standardized section of the scale in future revisions of the instrument.

From an individual's perspective, a systematic endorsement of supports needs assessment, planning, and delivery would encourage support teams to (a) align assessed support needs to specific support strategies, (b) specify support objectives for the provision of supports, and (c) identify the support team members who are responsible for implementing an individualized support plan (see Thompson et al., 2009). This approach is much different than specifying behavioral objectives that have historically been at the heart of individual plans and have unintentionally laid the onus on the individual to change. The onus shifts to members of the support team who are charged with taking responsibility for implementing support strategies. From an organization or system's perspective, a systematic approach to addressing support needs provides three essential functions: (a) it organizes potential support strategies into a system through which individualized supports can be planned and implemented based on the individual's 
assessed support needs; (b) it provides a framework for coordinating the procurement and application of individualized supports across the sources of support; and (c) it provides a framework for evaluating the impact of individualized supports on the individual's functioning and personal outcomes.

Of course, the extent to which a systematic, comprehensive approach to support needs assessment and provision results in improved quality of life for people with ID/DD will be contingent on many factors. ID/DD is a multifaceted field - it is certainly a field influenced by scientific knowledge claims, but it is also a field influenced by professional competence, political will, available resources, and countless other factors. The role of researchers in an applied field is to generate scientific knowledge claims so that public policy and professional practices are not based on false understandings. This investigation showed that there is an empirical basis for understanding people with ID/DD by their intensity of their support needs and classifying people into support need categories. Also, this investigation showed that there are specific, but related, dimensions of support needs on which to base assessment, measurement, and planning efforts. These knowledge claims provide important considerations for those who strive to transform the current system of service delivery to a system of supports facilitation.

\section{Limitations}

As was mentioned in Chapter 1, although data on which this investigation was based was comprehensive, no pretense was made that all potentially relevant data were available. For instance, comprehensive personal competence data (e.g., scores from multiple adaptive behavior scales and IQ scores) would have been helpful to provide a more thorough investigation into the relationship between personal competence variables and support needs scores. Also, Harries et al.'s (2005) unidimensional factor model could have been tested more rigorously as they included more extensive adaptive behavior data in their analysis than were included in this study. In addition, it cannot be assumed that all relevant measures of support needs were included in the 
analyses. Other instruments may be measuring aspects of support needs that the SIS does not.

\section{Recommendations for Future Research}

There are four logical next steps for future research based on the results of this investigation.

1. The relationship between support needs measures and personal competence measures merits additional investigation. Replicating this study with more extensive personal competence information in the data set would be helpful to see if the 7-Factor Hierarchical Model of Support Needs holds up with the inclusion of personal competence variables. Harries et al.'s (2005) exploratory factor analysis suggested that the SIS and adaptive behavior scales were measuring the same construct. Through the application of goodness-of-fit tests, it would be interesting to test alternative models including a unidimensional model and models featuring adaptive behavior factors (see Thompson, McGrew, and Bruininks [1999] for a list of prior research on structural models of adaptive behavior) and Support Needs factors.

\section{Replicating the cluster analysis procedures used in this investigation with} additional measures of support needs and personal outcome variables. Although it is reasonable to believe that groups would still be distinguished from one another based on relative intensity of support needs, the inclusion of additional variables may provide more nuanced divisions. For instance, detailed behavioral and medical data could show differences between people who need relatively intense supports due to exceptional medical problems or challenging behaviors. The sections of the SIS related to exceptional medical and behavioral support needs provided data that lack sufficient variance (the vast majority of people had scores of " 0 " for a medical and behavioral item) to be useful in cluster analysis. More sensitive medical and behavioral data would be helpful in future studies.

\section{Replicating the factor analysis procedures used in this investigation with}

additional support needs measures. Just as the inclusion of $\mathrm{P} \& \mathrm{~A}$ supplemental scale data 
resulted in the emergence of a new factor, it is conceivable that if data were available from the other support needs assessment scales mentioned in Chapter 2 (i.e., the I-CAN, the NC-SNAP, the SNAP), a structural model different than the 7-Factor Hierarchical Model may have been supported. It would be interesting to see if the factor structure would remain the same if additional data were added to the analyses.

\section{Investigating the possibility of creating a shorter version of the SIS. The SIS}

usually takes at least an hour to administer, and a shorter version of it that provided essential information for specific purposes would be helpful. Although there would be many good arguments as to why all items on the SIS should be assessed when using the scale for planning purposes, there may be other purposes where a shortened version is preferred (e.g., data for annual resource allocation decisions, data for reevaluation). Examining the factor loadings for the 7-Factor Hierarchical Model emerging from this study shows that some items load more heavily on latent factors than others. Further investigation of indicators that appear to have a strong empirical relationship to first-order support needs dimensions could provide an empirical basis for selecting items for a shorter version of the SIS.

\section{Chapter Summary}

Findings from the investigation were summarized and implications for professional practice, public policy, and future research were discussed. First, the groupings that emerged from the cluster analytic procedures were described in detail, as were the latent factors emerging from the application of factor analytic procedures. Next, the findings were discussed in terms of relevance to the six national goals published in 2005 that were identified by over 250 experts in the field of ID/DD at the National Goals Conference. The chapter ended with a discussion of the investigation's limitations and recommendations for future research. 


\section{REFERENCES}

AAIDD. (2013a). Supports Intensity Scale information. Retrieved from http://aaidd.org/docs/default-source/sis-docs/latestsispresentation.pdf?sfvrsn=2

AAIDD. (2013b). International SIS use. Retrieved from http://aaidd.org/sis/international\#.UdubCxawXac

AAIDD. (2013c). Benefits of the Supports Intensity Scale. Retrieved from http://aaidd.org/sis/product-info/benefits\#.UducUxawXac

AAIDD. (2013d). Why SIS is unique? Retrieved from http://aaidd.org/sis/product-info/whatmakes-sis-unique\#.Uduc8hawXac

AAIDD. (2013e). Frequently asked questions. Retrieved from http://www.siswebsite.org/page.ww?section=Articles\&name $=$ Most + Frequently + Asked + Qu estions+on+SIS

Agosta, J., Fortune, J., Kimmich, M., Melda, K., Smith, D., Auerbach, K., \& Taub, S. (2009). Ten issues for states to consider in implementing individual or level-based budget allocations. Retrieved from http://www.hsri.org/publication/ten-issues-for-states-to-consider-inimplementing-individual-or-level-/

Al-Yagon, M. (2012). Subtypes of attachment security in school-age children with learning disabilities. Learning Disability Quarterly, 35(3), 170-183.

Aldenderfer, M. S., \& Blashfield, R. K. (1984). Cluster analysis. London, UK: Sage.

Almqvist, L. (2006). Patterns of engagement in young children with and without developmental delay. Journal of Policy and Practice in Intellectual Disabilities, 3(1), 65-75.

APA. (1994). Diagnostic and statistical manual of mental disorders (4th ed.). Washington, DC: American Psychiatric Association.

APA. (2000). Diagnostic and statistical manual of mental disorders (4th ed., text rev.). Washington, DC: American Psychiatric Association.

Arc, The. (2011). Still in the shadows with their future uncertain: A report on family and individual needs for disability supports (FINDS). Retrieved from http://www.thearc.org/document.doc?id=3672

Arias, B., Verdugo, M. A., Navas, P., \& Gomez, L. E. (2013). Factor structure of the construct of adaptive behavior in children with and without intellectual disability. International Journal of Clinical and Health Psychology, 13, 155-166. 
Aricak, O. T., \& Oakland, T. (2010). Multigroup confirmatory factor analysis for the teacher form, ages 5 to 21, of the Adaptive Behavior Assessment System - II. Journal of Psychoeducational Assessment, 28, 578-584. doi: 10.1177/0734282909350209

Arnold, S. R. C., Riches, V. C., Parmenter, T. R., \& Stancliffe, R. J. (2009). The I-CAN: Using eHealth to get people the support they need. Electronic Journal of Health Informatics, 4(1), e4.

Arnold, S. R. C., Riches, V. C., \& Stancliffe, R. J. (2011). Intelligence is as intelligence does: Can additional support needs replace disability? Journal of Intellectual \& Developmental Disability, 36, 254-258. doi:10.3109/13668250.2011.617732

Autism Society. (2012). Facts and statistics. Retrieved from http://www.autismsociety.org/about-autism/facts-and-statistics.html

Bach, M. (2007). Changing perspectives on developmental disabilities. In I. Brown \& M. Percy (Eds.), A comprehensive guide to intellectual \& developmental disabilities (pp. 35-44). Baltimore, MD: Paul H. Brookes.

Bailey, K. D. (1994). Typologies and taxonomies. London, UK: Sage.

Baker, J. K., Seltzer, M. M., \& Greenberg, J. S. (2012). Behaviour problems, maternal internalizing symptoms and family relations in families of adolescents and adults with fragile X syndrome. Journal of Intellectual Disability Research, 56, 984-995. doi: 10.1111/j.1365-2788.2012.01580.x

Baumeister, A. A. (2006). Mental retardation: confusing sentiment with science. In H. N. Switzky \& S. Greenspan (Eds.), What is Mental Retardation? (pp. 93-124). Washington, DC: American Association on Mental Retardation.

Beirne-Smith, M., Ittenbach, R. F., \& Patton, J. (2006). Mental retardation: An introduction to intellectual disabilities (7th ed.). Upper Saddle River, NJ: Pearson Merrill.

Bergman, A. (n.d.). A guide to establishing an activity center for mentally retarded persons. Retrieved from http://mn.gov/mnddc/parallels2/pdf/70s/76/76-GEA-NAC.pdf

Biklen, D. (1988). The myth of clinical judgment. Journal of Social Issues, 44(1), 127-140.

Bitsika, V., Sharpley, C. F., \& Orapeleng, S. (2008). An exploratory analysis of the use of cognitive, adaptive and behavioural indices for cluster analysis of ASD groups. Journal of Intellectual Disability Research, 52, 973-985. doi: 10.1111/j.1365-2788.2008.01123.x

Blatt, B., \& Kaplan, F. (1966). Christmas in purgatory: A photographic essay on mental retardation. Boston, MA: Allyn \& Bacon.

Boan, C. H., \& Harrison, P. L. (1997). Adaptive behavior assessment and individuals with mental retardation. In R. L. Taylor (Ed.), Assessment of individuals with mental retardation (pp. 33-54). San Diego, CA: Singular. 
Bollen, K. A., \& Lennox, R. (1991). Conventional wisdom on measurement: A structural equation perspective. Psychological Bulletin. 110, 305-314.

Bollen, K. A., \& Long, J. S. (1993). Testing structural equation models. London, UK: Sage.

Bossaert, G., Kuppens, S., Buntinx, W., Molleman, C., Van den Abeele, A., \& Maes, B. (2009). Usefulness of the Supports Intensity Scale (SIS) for persons with other than intellectual disabilities. Research in Developmental Disabilities, 30, 1306-1316.

Boström, P. K., Broberg, M., \& Bodin, L. (2011). Child's positive and negative impacts on parents - A person-oriented approach to understanding temperament in preschool children with intellectual disabilities. Research in Developmental Disabilities, 32(5), 1860-1871.

Braddock, D., Hemp, R., \& Rizzolo, M. C. (2008). State of the States in developmental disabilities 2013. Washington, DC: American Association on Intellectual and Developmental Disabilities.

Braddock, D., Hemp., R., Rizzolo, M. C., Tanis, E. S., Haffer, L., Lulinski, A., \& Wu, J. (2013). State of the States in developmental disabilities 2013. Washington, DC: American Association on Intellectual and Developmental Disabilities.

Breen, P., \& Richman, G. (1979). Evolution of the developmental disabilities concept. In R. Weigerink \& J. W. Pelosi (Eds.), Developmental disabilities: The DD movement (pp. 3-6). Baltimore, MD: Paul H. Brookes.

Brown, H. K., Ouellette-Kuntz, H., Bielska, I., \& Elliott, D. (2009). Choosing a measure of support need: Implications for research and policy. Journal of Intellectual Disability Research, 53, 949-954. doi:10.1111/j.1365-2788.2009.01216.x

Brown, I., \& Radford, J. P. (2007). Historical overview of intellectual and developmental disabilities. In I. Brown \& M. Percy (Eds.), A comprehensive guide to intellectual \& developmental disabilities (pp. 17-34). Baltimore, MD: Paul H. Brookes.

Brown, J. D., \& Rodger, S. (2009). Children with disabilities: Problems faced by foster parents. Children and Youth Services Review, 31, 40-46. doi: 10.1016/j.childyouth.2008.05.007

Browne, M. W., \& Cudeck, R. (1993). Alternative ways of assessing model fit. In K. A. Bollen \& J. S. Long (Eds.), Testing structural equation models (pp. 136-162), Newbury Park, CA: Sage.

Bruininks, R. H., Hill, B. K., Weatherman, R. F., \& Woodcock, R. W. (1986). Inventory for Client and Agency Planning. Itasca, IL: Riverside.

Bruininks, R. H., Thurlow, M., \& Gilman, C. J. (1987). Adaptive behavior and mental retardation. Journal of Special Education, 21, 69-88.

Bryant, B. R. (1997). Intelligence testing. In R. L. Taylor (Ed.), Assessment of individuals with mental retardation (pp. 13-32). San Diego, CA: Singular. 
Buntinx, W. (2008). The Dutch version of the SIS. In R. L. Schalock, J. R. Thompson, \& M. J. Tassé (Eds.), Psychometric properties of the Supports Intensity Scale (pp. 6-9). Washington, DC: American Association on Intellectual and Developmental Disabilities.

Buntinx, W. (2011). Supports Intensity Scale psychometric properties: Similarities and differences between original U.S. versions and Dutch, Italian, French, and Spanish translations. Lecture presented on June 9, 2011 at the SIS Post-conference Workshop at the 35th Annual Meeting of the American Association on Intellectual and Developmental Disabilities, St. Paul, MN.

Buntinx, W. H. E., \& Schalock, R. L. (2010). Models of disability, quality of life, and individualized supports: Implications for professional practice in intellectual disability. Journal of Policy and Practice in Intellectual Disability, 7(4), 283-294.

Butterworth, J. (2002). Programs to supports. In R. L. Schalock, P. C. Baker, \& M. D. Croser (Eds.), Embarking on a new century: Mental retardation at the end of the 20th century (pp. 83-100). Washington, DC: American Association on Mental Retardation.

Carroll, J. B. (1993). Human cognitive abilities: A survey of factor-analytic studies. Cambridge, MA: Cambridge University Press.

CDHS. (2013). Support level determination / algorithm. Retrieved from http://www.colorado.gov/cs/Satellite/CDHS-VetDis/CBON/1251586991108

Centre for Disability Studies. (2012a). Instrument for the Classification and Assessment of Support Needs. Retrieved from http://www.i-can.org.au/index.php

Centre for Disability Studies. (2012b). I-CAN Further Info. Retrieved from http://www.ican.org.au/info.php

Chou, Y. C., Lee, Y. C., Chang, S. C., \& Yu, A. P. (2013). Evaluating the supports intensity scale as a potential assessment instrument for resource allocation for persons with intellectual disability. Research in Developmental Disabilities, 34, 2056-2063. doi: 10.1016/j.ridd.2013.03.013

Cicchetti, D. V., \& Sparrow, S. A. (1981). Developing criteria for establishing interrater reliability of specific items: Applications to assessment of adaptive behavior. American Journal of Mental Deficiency, 86(2), 127-137.

Cimera, R. E. (2011). Does being in sheltered workshops improve the employment outcomes of supported employees with intellectual disabilities? Journal of Vocational Rehabilitation, 35, 21-27. doi: 10.3233/JVR-2011-0550

Claes, C., Van Hove, G., van Loon, J., Vandevelde, S., \& Schalock, R. L. (2009). Evaluating the inter-respondent (consumer vs. staff) reliability and construct validity (SIS vs. Vineland) of the Supports Intensity Scale on a Dutch sample. Journal of Intellectual Disability Research, 53, 329-338. doi:10.1111/j.1365-2788.2008.01149.x 
Claes, C., Van Hove, G., Vandevelde, S., van Loon, J., \& Schalock, R. L. (2010). Personcentered planning: Analysis of research and effectiveness. Intellectual and Developmental Disabilities, 48(6), 432-453.

Claes, C., Van Hove, G., Vandevelde, S., van Loon, J., \& Schalock, R. (2012). The influence of supports strategies, environmental factors, and client characteristics on quality of liferelated personal outcomes. Research in Developmental Disabilities, 33, 96-103.

Clark, G. M., \& Kolstoe, O. P. (1995). Career development and transition education for adolescents with disabilities (2nd ed.). Needham Heights, MA: Allyn \& Bacon.

Clausen, J. (1972). The continuing problem of defining mental deficiency. Journal of Special Education, 6, 97-106.

Clay-Adkins, S. L. (2004). Reliability and validity of the Supports Intensity Scale (Doctoral dissertation). Retrieved from ProQuest. (3128272)

Community Support Network. (2013). Supports Intensity Scale: Identifying supports for success. Retrieved from http://www.csni.org/supports-intensity-scale/

Copeland, S. R., \& Cosbey, J. (2008). Making progress in the general curriculum: Rethinking effective instructional practices. Research \& Practice for Persons with Severe Disabilities, $33(4), 214-227$.

Council on Quality and Leadership. (2012). Application for services for people with disabilities. Retrieved from http://www.thecouncil.org/pceguidedisability.aspx

Cruz, M., Perez, M. d. C., Jenaro, C., Flores, N., \& Vega, V. (2013). Identification of the support needs of individuals with severe mental illness using the Supports Intensity Scale. Latin American Journal of Nursing, 21(5), 1137-1143.

Danforth, S., \& Rhodes, W. C. (1997). Deconstructing disability: A philosophy for inclusion. Remedial and Special Education, 18, 357-366.

Davison, H. (2005). The Supports Intensity Scale: Book review. Journal of Intellectual Disability Research, 49(8), 636.

Debachere, M. C. (1995). Problems in obtaining grey literature. IFLA Journal-International Federation of Library Associations, 21(2), 94-98.

DeJong, J., \& Donders, J. (2010). Cluster subtypes on the California Verbal Learning TestSecond Edition (CVLT-II) in a traumatic brain injury sample. Journal of Clinical and Experimental Neuropsychology, 32(9), 953-960.

Doll, E. A. (1935). A genetic scale of social maturity. American Journal of Orthopsychiatry, 5, 180-188.

Doll, E. A. (1941). The essentials of an inclusive concept of mental deficiency. American Journal of Mental Deficiency, 46, 214-219. 
DPW. (2013). Supports Intensity Scale and PA Plus (SIS $\left.{ }^{\mathrm{TM}}\right)$. Retrieved from http://www.dpw.state.pa.us/foradults/intellectualdisabilitiesservices/supportintensityscalean dpaplussis/

Drew, C. J., \& Hardman, M. L. (2007). Intellectual disabilities across the lifespan (9th ed.). Upper Saddle River, NJ: Pearson.

DSHS. (2013). The DDD assessment. Retrieved from http://www.dshs.wa.gov/ddd/CAP.shtml

Durkin, M. S., \& Stein, Z. A. (1996). Classification of mental retardation. In J. W. Jacobson \& J. A. Mulick (Eds.), Manual of diagnosis and professional practice in mental retardation (pp. 101-136). Washington, DC: American Psychological Association.

Dykens, E. M., Hodapp, R. M., \& Finucane, B. M. (2000). Genetics and mental retardation syndromes: A new look at behavior and interventions. Baltimore, MD: Paul H. Brookes.

Edgerton, R. B. (1993). The cloak of competence (2nd ed.). Los Angeles, LA: University of California Press.

Edgerton, R. B., \& Bercovici, S. (1976). The cloak of competence: Years later. American Journal of Mental Deficiency, 80, 485-497.

Everitt, B. S., Landau, S., Leese, M., \& Stahl, D. (2011). Cluster analysis (5th ed.). London, UK: Wiley.

Fahey, A., Walsh, P. N., Emerson, E., \& Guerin, S. (2010). Characteristics, supports, and quality of life of Irish adults with intellectual disability in life-sharing residential communities. Journal of Intellectual \& Developmental Disability, 35(2), 66-76.

Farmer, T. W., Hall, C. M., Weiss, M. P., Petrin, R. A., Meece, J. L., \& Moohr, M. (2011). The school adjustment of rural adolescents with and without disabilities: Variable and personcentered approaches. Journal of Child \& Family Studies, 20, 78-88. doi: 10.1007/s10826$010-9379-2$

Flynn, J. R. (2007). What if intelligence? Beyond the Flynn Effect. New York, NY: Cambridge.

Fortune, J., Agosta, J., \& Bershadsky, J. (2011). 2011 Validity and reliability results regarding the SIS. Retrieved from

www.hsri.org/files/uploads/publications/SIS_Reliability_and_Validity_2011.pdf

Fortune, J., Auerbach, K., Melda, K., Agosta, J., \& Smith, D. (2009). Colorado Supported Living Services (SLS) Waiver: Equitably serving people with developmental disabilities with SISinformed spending caps using uniform methodology for funding allocation. Retrieved from http://www.colorado.gov/cs/Satellite?blobcol=urldata\&blobheadername1=ContentDisposition\&blobheadername2 $=$ Content Type\&blobheadervalue $1=$ inline $\% 3 \mathrm{~B}+$ filename $\% 3 \mathrm{D} \% 22$ Supported + Living + Services + Waiv er + Spending + Caps + HSRI + Final + Report $+i+$ February $+9 \% 2 C+2009+$.pdf $\% 22 \&$ blobheader value $2=$ application $\% 2$ Fpdf\&blobkey $=\mathrm{id} \&$ blobtable $=$ MungoBlobs $\&$ blobwhere $=125181804$ $1643 \&$ ssbinary $=$ true 
Gearheart, B. R., \& Litton, F. W. (1975). Trainable retarded: A foundation approach. Saint Louis, MO: C.V. Mosby.

Giné, C. (2008). Catalan translation of the Supports Intensity Scale. In R. L. Schalock, J. R. Thompson, \& M. J. Tassé (Eds.), International implementation of the Supports Intensity Scale (pp. 7-8). Washington, DC: American Association on Intellectual and Developmental Disabilities.

Gould, S. J. (1981). The mismeasure of man. New York, NY: W. W. Norton.

Greenspan, S. (1997). Dead manual walking? Why the 1992 AAMR definition needs redoing. Education and Training in Mental Retardation and Developmental Disabilities, 32(3), 179190.

Greenspan, S., \& Granfield, J. M. (1992). Reconsidering the construct of mental retardation: Implications of a model of social competence. American Journal on Mental Retardation, 96(4), 442-453.

Greenspan, S., Loughlin, G., \& Black, R. S. (2001). Credulity and gullibility in people with developmental disorders: A framework for future research. International Review of Research in Mental Retardation, 24(1), 101-135.

Groce, N. E. (1996). Parent advocacy for disabled children and the disability rights movement: Similar movements, different trajectories. Retrieved from http://eprints.ucl.ac.uk/15151/1/15151.pdf

Grossman, H. J. (1973). A manual on terminology and classification in mental retardation (rev. ed.). Washington, DC: American Association on Mental Deficiency.

Grossman, H. J. (1983). Classification in mental retardation (Rev. ed.). Washington, DC: American Association on Mental Deficiency.

Guscia, R., Harries, J., Kirby, N., Nettelbeck, T., \& Taplin, J. (2005). Reliability of the Service Need Assessment Profile (SNAP): A measure of support for people with disabilities. Journal of Intellectual \& Developmental Disability, 30, 24-30. doi:10.1080/13668250500033144

Guscia, R., Harries, J., Kirby, N., Nettelbeck, T., \& Taplin, J. (2006). Construct and criterion validities of the Service Need Assessment Profile (SNAP): A measure of support for people with disabilities. Journal of Intellectual \& Developmental Disability, 31, 148-155. doi:10.1080/13668250600876459

Hamelin, J. P., Frijters, J., Griffiths, D., Condillac, R., \& Owen, F. (2011). Meta-analysis of deinstitutionalization adaptive behavior outcomes: Research and clinical implications. Journal of Intellectual \& Developmental Disability, 36, 61-72. doi:10.3109/13668250.2010.544034

Harries, J., Guscia, R., Kirby, N., Nettelbeck, T., \& Taplin, J. (2005). Support needs and adaptive behaviors. American Journal on Mental Retardation, 110(5), 393-404. 
Harris, J. C. (2006). Intellectual disability: Understanding its development, causes, classification, evaluation, and treatment. New York, NY: Oxford.

Heber, R. (1959). A manual on terminology and classification in mental retardation: A monograph supplement to the American Journal on Mental Deficiency, 64 (Monograph Suppl.).

Heber, R. (1961). A manual on terminology and classification on mental retardation. Washington, DC: American Association on Mental Deficiency.

Hennike, J. M. (2002). DD-SNAP. Exceptional Parent, 32(9), 56-57.

Hennike, J. M., Myers, A. M., Realon, R. E., \& Thompson, T. J. (2006). Development and validation of a needs-assessment instrument for persons with developmental disabilities. Journal of Developmental and Physical Disabilities, 18(2), 201-217.

Hodapp, R. M. (1995). Definitions in mental retardation: Effects on research, practice, perceptions. School Psychology Quarterly, 10(1), 24-28.

Hu, L., \& Bentler, P. M. (1999). Cutoff criteria for fit indexes in covariance structure analysis: Conventional criteria versus new alternatives. Structural Equation Modeling, 6(1), 1-55.

Hubert, J. (2011). "My heart is always where he is". Perspectives of mothers of young people with severe intellectual disabilities and challenging behaviour living at home. British Journal of Learning Disabilities, 39, 216-224. doi: 10.1111/j.1468-3156.2010.00658.x

Hunt, E. B. (2011). Human intelligence. New York, NY: Cambridge University Press.

IES. (2012). Funding opportunities. Retrieved from http://ies.ed.gov/funding/grantsearch/details.asp?ID=1175

Illinois Department of Human Services. (2012). Shapiro developmental center profile. Retrieved from http://www.dhs.state.il.us/page.aspx?item $=58714$

Jacobson, J. W., \& Mulick, J. A. (1996). Manual of diagnosis and professional practice in mental retardation. Washington, DC: American Psychological Association.

Jenaro, C., Cruz, M., Perez, M. d. C., Flores, N. E., \& Vega, V. (2011). Utilization of the Supports Intensity Scale with psychiatric populations: Psychometric properties and utility for service delivery planning. Archives of Psychiatric Nursing, 25(5), e9-e17.

Jöreskog, K. G., \& Sörbom, D. (2006). LISREL 8.8 for Windows (Computer software). Skokie, IL: Scientific Softward International, Inc.

Kail, R. V., \& Pellegrino, J. W. (1985). Human intelligence: Perspectives and prospects. New York, NY: Freeman.

Kim, J., \& Mueller, C. W. (1978). Introduction to factor analysis: What it is and how to do it. London, UK: Sage. 
Kimmich, M., Agosta, J., Fortune, J., Smith, D., Melda, K., Auerbach, K., \& Taub, S. (2009). Developing individual budgets and reimbursement levels using the Supports Intensity Scale. Retrieved from http://www.hsri.org/files/uploads/publications/Individual_Budgets_Using_SIS_FINAL.pdf

Kirk, S. A. (1972). Educating exceptional children. Boston, NY: Houghton Mifflin.

Klein, P. D. (1997). Multiplying the problems of intelligence by eight: A critique of Gardner's theory. Journal of Education, 22, 377-394.

Kollock, P., \& O'Brien, J. (1994). The production of reality: Essays and readings in social psychology. Newbury, CA: Pine Forge Press.

Koritsas, S., Iacono, T., Hamilton, D., \& Leighton, D. (2008). The effect of active support training on engagement, opportunities for choice, challenging behaviour and support needs. Journal of Intellectual \& Developmental Disabilities, 33, 247-256. doi: $10.1080 / 13668250802282944$

Kuhn, T. S. (1962). The structure of scientific revolutions. Chicago, IL: University of Chicago.

Kuppens, S., Bossaert, G., Buntinx, W., Molleman, C., Van den Abbeele, A., \& Maes, B. (2010). Factorial validity of the Supports Intensity Scale (SIS). American Journal on Intellectual and Developmental Disabilities, 115(4), 327-339.

Lakin, K. C., \& Stancliffe, R. J. (2007). Residential supports for persons with intellectual and developmental disabilities. Mental Retardation and Developmental Disabilities Research Reviews, 13, 151-159.

Lakin, K. C., \& Turnbull, A. P. (2005). National goals and research for people with intellectual and developmental disabilities. Washington, DC: American Association on Intellectual and Developmental Disabilities.

Lamoureux-Hebert, M., \& Morin, D. (2008). French translation of the Supports Intensity Scale. In R. L. Schalock, J. R. Thompson, \& M. J. Tassé (Eds.), International implementation of the Supports Intensity Scale (pp. 3-4). Washington, DC: American Association on Intellectual and Developmental Disabilities.

Lamoureux-Hebert, M., Morin, D., \& Crocker, A. (2010). Support needs of individuals with mild and moderate intellectual disabilities and challenging behaviors. Journal of Mental Health Research in Intellectual Disability, 3, 67-84, doi: 10.1080/19315861003650558

Larson, S. A., Lakin, K. C., Salmi, P., Scotte, N., \& Webster, A. (2010). Children and youth with intellectual and developmental disabilities living in congregate care settings (1977-2009): Healthy people 2010 objective 6.7b outcomes. Intellectual and Developmental Disabilities, 48, 396-400. doi: 10.1352/1934-9556-48.5.396

Lattin, J. M., Carroll, J. D., \& Green, P. E. (2003). Analyzing multivariate data. Pacific Grove, CA: Thomson. 
LeVelle, J. A., \& Meche, S. (2008). Individual resource allocation in Louisiana. In R. L. Schalock, J. R. Thompson, \& M. J. Tassé (Eds.), Resource allocation and the Supports Intensity Scale: Four papers on issues and approaches (pp. 22-23). Washington, DC: American Association on Intellectual and Developmental Disabilities.

Lippmann, W. (1922). The abuse of the tests. The New Republic, 32(415), 297-298.

Loew, S. A. (2005). Test review of the Supports Intensity Scale. In R. A. Spies \& B. S. Plake (Eds.), The sixteenth mental measurements yearbook (pp. 999-1001). Lincoln, NE: Buros Institute of Mental Measurements.

Long, J. S. (1983). Confirmatory factor analysis: A preface to LISREL. Beverly Hills, CA: Sage.

Luckasson, R., Borthwick-Duffy, S., Buntinx, W. H. E., Coulter, D. L., Craig, E. M., Reeve, A., ... Tassé, M. J. (2002). Mental retardation: Definition, classification, and systems of supports (10th ed.). Washington, DC: American Association on Mental Retardation.

Luckasson, R., Coulter, D. L., Polloway, E. A., Reese, S., Schalock, R. L., Snell, M. E., ... Stark, J. A. (1992). Mental retardation: Definition, classification, and systems of supports (9th ed.). Washington, DC: American Association on Mental Retardation.

Luckasson, R., \& Reeve, A. (2001). Naming, defining, and classifying in mental retardation. Mental Retardation, 39, 47-52.

MacMillan, D. L. (1977). Mental retardation in school and society. Boston, MA: Little, Brown and Company.

MacMillan, D. L. (1982). Mental retardation in school and society (2nd ed.). Boston, MA: Little, Brown.

MacMillan, D. L., Gresham, F. M., Bocian, K. M., \& Lambros, K. M. (1998). Current plight of borderline students: Where do they belong? Education \& Training in Mental Retardation \& Developmental Disabilities, 33(2), 83-94.

MacMillan, D. L., Gresham, F. M., \& Siperstein, G. N. (1993). Conceptual and psychometric concerns about the 1992 AAMR definition of mental retardation. American Journal on Mental Retardation, 98(3), 325-335.

MacMillan, D. L., Gresham, F. M., \& Siperstein, G. N. (1995). Heightened concerns over the 1992 AAMR definition: Advocacy versus precision. American Journal on Mental Retardation, 100(1), 87-97.

McGrew, K. S. \& Flanagan, D. P. (1998). The Intelligence Test Desk Reference (ITDR): Gf-Gc Cross-Battery Assessment. Boston, MA: Allyn \& Bacon.

Mercer, J. R. (1973). Labelling the mentally retarded. Berkeley, CA: University of California.

Mercer, J. R. (1979). SOMPA: Technical and conceptual manual. New York, NY: Psychological Corporation. 
Miller, P. (2010). Phonological, orthographic, and syntactic awareness and their relation to reading comprehension in prelingually deaf individuals: What can we learn from skilled readers? Journal on Developmental and Physical Disabilities, 22, 549-580. doi: $10.1007 / \mathrm{s} 10882-010-9195-\mathrm{z}$

Morales, G. E., Lopez, E. O., \& Mullet, E. (2011). Acceptability of sexual relationships among people with learning disabilities: Family and professional caregivers' views in Mexico. Sexuality \& Disability, 29, 165-174. doi: 10.1007/s1195-011-9201-0

Morin, D., \& Cobigo, V. (2008). The French version of the Supports Intensity Scale. In R. L. Schalock, J. R. Thompson, \& M. J. Tassé (Eds.), Psychometric properties of the Supports Intensity Scale (pp. 3-4). Washington, DC: American Association on Intellectual and Developmental Disabilities.

Moseley, C., Gettings, R., \& Cooper, R. (2003). Having it your way: Understanding state individual budgeting strategies. Alexandria, VA: National Association of State Directors of Developmental Disabilities Services.

Murdick, N. L., Gartin, B. C., \& Crabtree, T. (2007). Special education law (2nd ed.). Upper Saddle River, NJ: Pearson.

Murdoch Center Foundation. (2013). DD-SNAP: Products. Retrieved from http://www.murdochfoundation.org/products.htm

Mutua, K., Siders, J., \& Bakken, J. P. (2011). History of intellectual disabilities. Advances in special education, 21, 89-117. doi:10.1108/S0270-4013(2011)0000021008

Myers, P. I., \& Hammill, D. D. (1990). Learning disabilities: Basic concepts, assessment practices, and instructional strategies. Austin, TX: Pro-Ed.

National Down Syndrome Congress. (2012). Facts about Down syndrome. Retrieved from http://ndsccenter.org/resources/general-information/facts-about-down-syndrome/

National Institute of Health. (2000). Phenylketonuria: Screening and management. Retrieved from http://consensus.nih.gov/2000/2000phenylketonuria113html.htm

National Institute of Neurological Disorders and Stroke. (2012). Shaken Baby Syndrome information page. Retrieved from

http://www.ninds.nih.gov/disorders/shakenbaby/shakenbaby.htm

Navigant. (2007). Colorado Division for Developmental Disabilities comprehensive waiver proposed rates and impact analysis. Retrieved from

http://cospl.coalliance.org/fedora/repository/co:1110/hu252w362007internet.pdf

NCDHHS. (2013a). History and development of NC SNAP. Retrieved from http://www.ncdhhs.gov/mhddsas/providers/NCSNAP/historyanddevelopment.htm

NCDHHS. (2013b). Examiner certification training. Retrieved from http://www.ncdhhs.gov/mhddsas/providers/NCSNAP/examinercert.htm 
NCDHHS. (2013c). Community alternatives program for persons with mental retardation/developmental disabilities. Retrieved from http://www.ncdhhs.gov/mhddsas/providers/CAPMRDD/index.htm

NCDHHS. (2013d). CAP-MR/DD: Support Planning Process Supports Intensity Scale. Retrieved from http://www.ncdhhs.gov/mhddsas/providers/CAPMRDD/SIS/

NDA. (2011). Introduction of individual budgets as a resource allocation system for disability services in Ireland. Retrieved from http://www.nda.ie/CntMgmtNew.nsf/DCC524B4546ADB3080256C700071B049/DE4CF1 0DC964B4FA8025789A004B492E?OpenDocument

Newschaffer, C. J., Falb, M. D., \& Gurney, J. G. (2005). National autism prevalence trends from United States Special Education data. Pediatrics, 115, 277-282.

Nihira, K. (1999). Adaptive behavior: A historical overview. In R. L. Schalock (Ed.), Adaptive behavior and its measurement: Implications for the field of mental retardation (pp. 7-14). Washington, DC: American Association on Mental Retardation.

Nirje, B. (1969). The normalization principle and its human management implications. In $\mathrm{R}$. Kugel \& W. Wolfensberger (Eds.), Changing patterns in residential services for the mentally retarded (pp. 179-195). Washington, DC: President's Committee on Mental Retardation.

Norris, M., \& Lecavalier, L. (2010). Evaluating the use of exploratory factor analysis in developmental disability psychological research. Journal on Autism and Developmental Disorders, 40, 8-20. doi: 10.1007/s10803-009-0816-2

Nunez, J. C., Gonzalez-Pienda, J. A., Rodriguez, C., Valle, A., Gonzalez-Cabanach, R., \& Rosario, P. (2011). Multiple goals perspective in adolescent students with learning difficulties. Learning Disability Quarterly, 34, 273-286. doi: 10.1177/0731948711421763

Nygren, M. (2011, December). Midwinter Leadership Meeting. Meeting of the American Association on Intellectual and Developmental Disabilities, Orlando, FL.

Perske, R. (1972). The dignity of risk and the mentally retarded. Mental Retardation, 10, 24-27.

Pew Center on the States. (2010). Facing facts: Public attitudes and fiscal realities in five stressed states. Retrieved from http://www.pewstates.org/uploadedFiles/PCS_Assets/2010/Public_Attitudes_and_Fiscal_R ealities_in_Five_Stressed_States.pdf

Pittenger, D. J. (2005). Test review of the Supports Intensity Scale. In R. A. Spies \& B. S. Plake (Eds.), The sixteenth mental measurements yearbook (pp. 1001-1003). Lincoln, NE: Buros Institute of Mental Measurements.

Pledger, C. (2003). Discourse on disability and rehabilitation issues: Opportunities for psychology. American Psychologist, 58, 279-284. doi:10.1037/0003-066X.58.4.279 
Polloway, E. (1997). Developmental principles of the Luckasson et al. (1992) AAMR definition of mental retardation. Education and Training in Mental Retardation and Developmental Disabilities, 32, 174-178.

Polloway, E. A., Smith, J. D., Chamberlain, J., Denning, C. B., \& Smith, T. E. C. (1999). Levels of deficits or supports in the classification of mental retardation: Implementation practices. Education and Training in Mental Retardation and Developmental Disabilities, 34, 200 206.

Pope, A. M., \& Tarlov, A. R. (1991). Disability in America: Toward a national agenda for prevention. Washington, DC: National Academy Press.

Productivity Commission. (2011). Disability care and support. Retrieved from http://www.pc.gov.au/_data/assets/pdf_file/0012/111270/disability-support-volume1.pdf

Productivity Commission. (2012). Assessment tools. Retrieved from http://www.google.com/url?sa=t\&rct=j\&q=new\%20south\%20wales\%20australia\%20snap $\% 20$ support $\% 20$ needs \& source $=$ web $\& \mathrm{~cd}=1 \& \mathrm{ved}=0 \mathrm{CB} 8 \mathrm{QFjAA} \&$ url $=\mathrm{http} \% 3 \mathrm{~A} \% 2 \mathrm{~F} \% 2 \mathrm{Fww}$ w.pc.gov.au $\% 2 \mathrm{~F}$ _data $\% 2 \mathrm{Fassets} \% 2 \mathrm{Fword}$ _doc $\% 2 \mathrm{~F} 0013 \% 2 \mathrm{~F} 111343 \% 2 \mathrm{~F} 26$-disabilitysupportappendixb.doc\&ei=tTZmUMGGAdGfqwHO6oGYAw\&usg=AFQjCNGJaGILeoRgtpR9u HE8_TPaJL_O1w\&sig2=bGIZ14wnimS9zxeZM26W0g\&cad=rja

Prucher, F., \& Langfeldt, H. (2002). How German teachers in special education perceive and describe children with a learning disability. International Journal of Disability, Development and Education, 49, 399-411. doi: 10.1080/1034912022000028367.

Putnam, C. P., Carstens, C. C., Burleigh, E. N., Curtis, F. G., Cushing, G. D., Evans, E. G., ... Woodward, S. B. (1913). The menace of the feeble-minded in Massachusetts in institutions 1900: The need of a program. Retrieved from http://ia700408.us.archive.org/18/items/menaceoffeeblemi00mass/menaceoffeeblemi00mas s.pdf

Reschly, D. J., Myers, T. G., \& Hartel, C. R. (2002). Mental retardation: Determining eligibility for social security benefits. Washington, DC: National Academy Press.

Riches, V. C. (2003). Classification of support needs in a residential setting. Journal of Intellectual \& Developmental Disability, 28, 323-341. doi: 10.1080/1366825031000150982

Riches, V. C., Parmenter, T. R., Llewellyn, G., Hindmarsh, G., \& Chan, J. (2009). The reliability, validity and practical utility of measuring supports using the I-CAN instrument. Journal of Applied Research in Intellectual Disabilities, 22, 340-353. doi:10.1111/j.14683148.2008.00467.x

Roberts, G., Becker, H., \& Seay, P. (1997). A process for measuring adoption of innovation within the supports paradigm. Journal of the Association for Persons with Severe Handicaps, 22(2), $109-119$. 
Routh, D. K. (1996). Intellectual development. In J. W. Jacobson, \& J. A. Mulick (Eds.). Manual of diagnosis and professional practice in mental retardation (pp. 85-95). Washington, DC: American Psychological Association.

Schalock, R. L. (1999). Adaptive behavior and its measurement: Implications for the field of mental retardation. Washington, DC: American Association on Mental Retardation.

Schalock, R. L. (2004). The concept of quality of life: What we know and do not know. Journal of Intellectual Disability Research, 48(3), 203-216.

Schalock, R. L. (2011). The evolving understanding of the construct of intellectual disability. Journal of Intellectual \& Developmental Disability, 36(4), 227-237.

Schalock, R. L., Borthwick-Duffy, S., Bradley, V. J., Buntinx, W. H. E., Coulter, D. L., Craig, E. M., ... Yeager, M. H. (2010). Intellectual disability: Definition, classification, and systems of supports (11 th ed.). Washington, DC: American Association on Intellectual and Developmental Disabilities.

Schalock, R. L., Luckasson, R. A., Shogren, K. A., Borthwick-Duffy, S., Bradley, V., Buntinx, W. H. E., ... Yeager, M. H. (2007). The renaming of mental retardation: Understanding the change to the term intellectual disability. Intellectual and Developmental Disabilities, $45(2), 116-124$.

Schalock, R. L., Thompson, J. R., \& Tassé, M. J. (2008a). Psychometric properties of the Supports Intensity Scale. Washington, DC: American Association on Intellectual and Developmental Disabilities.

Schalock, R. L., Thompson, J. R., \& Tassé, M. J. (2008b). International implementation of the Supports Intensity Scale. Washington, DC: American Association on Intellectual and Developmental Disabilities.

Schalock, R. L., Thompson, J. R., \& Tassé, M. J. (2008c). Resource allocation and the Supports Intensity Scale: Four papers on issues and approaches. Washington, DC: American Association on Intellectual and Developmental Disabilities.

Schalock, R. L., Thompson, J. R., \& Tassé, M. J. (2008d). Relating Supports Intensity Scale information to individual service plans. Washington, DC: American Association on Intellectual and Developmental Disabilities.

Scheerenberger, R. C. (1983). A history of mental retardation. Baltimore, MD: Paul H. Brookes.

Scheerenberger, R. C. (1987). A history of mental retardation: A quarter century of promise. Baltimore, MD: Paul H. Brookes.

Severance, D. D., \& Campbell, E. M. (2008). What is a funding formula? In R. L. Schalock, J. R. Thompson, \& M. J. Tassé (Eds.), Resource allocation and the Supports Intensity Scale: Four papers on issues and approaches (pp. 7-10). Washington, DC: American Association on Mental Retardation. 
Shahbazian, N., Barati, M., Arian, P., \& Saadati, N. (2012). Comparison of complications of chorionic villus sampling and amniocentesis. International Journal of Fertility and Sterility, 5(4), 241-244.

Sleeter, C. E. (1986). Learning disability: The social construction of a special education category. Exceptional Children, 53(1), 46-54.

Smit, W., Sabbe, B., \& Prinzie, P. (2011). Reliability and validity of the Supports Intensity Scale (SIS) measured in adults with physical disabilities. Journal of Developmental and Physical Disabilities, 23, 277-287. doi: 10.1007/s10882-011-9227-3

Smith, J. D. (1997). Mental retardation as an educational construct: Time for a new shared view? Education and Training in Mental Retardation and Developmental Disabilities, 32(3), 167173.

Smith, G., \& Fortune, J. (2008). The Supports Intensity Scale and resource allocation. In R. L. Schalock, J. R. Thompson, \& M. J. Tassé (Eds.), Resource allocation and the Supports Intensity Scale: Four papers on issues and approaches (pp. 3-6). Washington, DC: American Association on Intellectual and Developmental Disabilities.

Smith, J. D., \& Wehmeyer, M. L. (2012). Good blood, bad blood: Science, nature, and the myth of the Kallikaks. Washington, DC: American Association on Intellectual and Developmental Disabilities.

Snell, M. E., Luckasson, R., Borthwick-Duffy, S., Bradley, V., Buntinx, W. H. E., Coulter, D. L., ... Yeager, M. H. (2009). Characteristics and needs of people with intellectual disability who have higher IQs. Intellectual and Developmental Disabilities, 47, 220-233. doi: $10.1352 / 1934-9556-47.3 .220$

Sobsey, D. (1987). Ecological inventory exemplars. Edmonton, Canada: University of Alberta.

Soenen, S., Berckelaer-Onnes, I. V., \& Scholte, E. (2009). Patterns of intellectual, adaptive and behavioral functioning in individuals with mild mental retardation. Research in Developmental Disabilities, 30(3), 433-444.

Special Olympics. (2013). R-word: Spread the word to end the word. Retrieved from http://www.r-word.org/

Stancliffe, R. J., \& Lakin, K. C. (2005). Cost and outcomes of community services for people with intellectual disabilities. Baltimore, MD: Paul H. Brookes.

Sternberg, R. J. (2005). The triarchic theory of successful intelligence. In D. P. Flanagan \& P. L. Harrison (Eds.), Contemporary intellectual assessment: Theories, tests, and issues (2nd ed.; pp. 103-119). New York, NY: Guilford.

Streissguth, A. (1997). Fetal Alcohol Syndrome: A Guide for families and communities. Baltimore, MD: Paul H. Brookes. 
Swanton, S., Walsh, S., O'Murchu, R., \& O'Flynn, P. (2010). A tool to determine support needs for community life. Learning Disability Practice, 13(8), 24-26.

Tanaka, J. S. (1993). Multifaceted conceptions of fit in structural equation models. In K. A. Long, \& J. S. Bollen (Eds.), Testing structural equation models (pp. 10-39). London, UK: Sage.

Tassé, M. J., \& Wehmeyer, M. L. (2010). Intensity of support needs in relation to co-occurring psychiatric disorders. Exceptionality, 18, 182-192. doi: 10.1080/09362835.2010.513922

Thompson, J. R. (2010). Introduction to the special issue: Support needs assessment and planning for children and adults with intellectual disabilities. Exceptionality, 18, 165-167. doi: $10.1080 / 09362835.2010 .513918$

Thompson, J. R., Bradley, V. J., Buntinx, W. H. E., Schalock, R. L., Shogren, K. A., Snell, M. E., \& Wehmeyer, M. L. (2009). Conceptualizing supports and the support needs of people with intellectual disability. Intellectual and Developmental Disabilities, 47(2), 135-146.

Thompson, J. R., Bryant, B., Campbell, E. M., Craig, E. M., Hughes, C., Rotholz, D. A., ... Wehmeyer, M. (2004a). Supports Intensity Scale (SIS). Washington, DC: American Association on Mental Retardation.

Thompson, J. R., Bryant, B., Campbell, E. M., Craig, E. M., Hughes, C., Rotholz, D. A., ... Wehmeyer, M. (2004b). The Supports Intensity Scale (SIS) Users manual. Washington, DC: American Association on Mental Retardation.

Thompson, J. R., \& Ezell, D. (2005). Naming, defining, and classifying the condition (formerly?) known as mental retardation. Journal of Social Work in Disability and Rehabilitation, 4, 523. doi: $10.1300 / J 198 v 04 n 03 \_03$

Thompson, J. R., Hughes, C., Schalock, R. L., Silverman, W., Tassé, M. J., Bryant, B., ... Campbell, E. M. (2002). Integrating supports in assessment and planning. Mental Retardation, 40(5), 390-405.

Thompson, J. R., McGrew, K. S., \& Bruininks, R. H. (1999). Adaptive and maladaptive behavior: Functional and structural characteristics. In R. L. Schalock (Ed.), Adaptive behavior and its measurement: Implications for the field of mental retardation (pp. 15-42). Washington, DC: American Association on Mental Retardation.

Thompson, J. R., McGrew, K. S., Johnson, D. R., \& Bruininks, R. H. (1999). Refining a multidimensional model of community adjustment through an analysis of postschool follow-up data. Exceptionality, 8(2), 73-99.

Thompson, J. R., Shogren, K. A., Wehmeyer, M. L., Chapman, T., Tassé, M. J., \& McLaughlin, C.A. (in press). The psychometric properties of the Supplemental Protection and Advocacy Scale of the SIS. Inclusion.

Thompson, J. R., Tassé, M. J., \& McLaughlin, C. A. (2008). Interrater reliability of the Supports Intensity Scale (SIS). American Journal on Mental Retardation, 113(3), 231-237. 
Thompson, J. R., \& Viriyangkura, Y. (2013). Supports and support needs. In M. L. Wehmeyer (Ed.), The Oxford handbook of positive psychology and disability. Retrieved from http://www.oxfordhandbooks.com/view/10.1093/oxfordhb/9780195398786.001.0001/oxfor dhb-9780195398786-e-031?rskey=tBhyge\&result $=4$

Thompson, J. R., \& Wehmeyer, M. L. (2008). Historical and legal issues in developmental disabilities. In H. P. Parette \& G. R. Peterson-Karlan (Eds.), Research-based practices in developmental disabilities (2nd ed; pp. 13-42). Austin, TX: Pro-Ed.

Thompson, J. R., Wehmeyer, M. L., \& Hughes, C. (2010). Mind the gap! Intellectual disability as individual-environment fit: Implications for students, teachers, and schools. Exceptionality, 18, 168-181. doi: 10.1080/09362835.2010.513919

Thompson, J. R., Wehmeyer, M. L., Hughes, C., Copeland, S. R., Little, T. D., Obremski, S., ... Tassé, M. J. (2012). Supports Intensity Scale for Children Field Test Version 2.0. Unpublished assessment instrument.

Thorndike, R. M., \& Thorndike-Christ, T. (2010). Measurement and evaluation in psychology and education (8th ed.). Boston, MA: Pearson.

Turnbull, A., Turnbull, H. R., Erwin, E. J., Soodak, L. C., \& Shogren, K. A. (2011). Families, professionals, and exceptionality: Positive outcomes through partnerships and trust (6th ed.). Upper Saddle River, NJ: Pearson.

Tymchuk, A. J., Lakin, L. K., \& Luckasson, R. (2001). The forgotten generation: The status and challenges of adults with mild cognitive limitations. Baltimore, MD: Paul H. Brookes.

United Nations. (1981). The right to self-determination: Historical and current development on the basis of United Nations instruments. Retrieved from http://www.cetim.ch/en/documents/cristescu-rap-ang.pdf

US Census Bureau. (2012). USA QuickFacts. Retrieved from http://quickfacts.census.gov/qfd/states/00000.html

U.S. Department of Education. (2011). 30 th Annual report to Congress on the implementation of the Individuals with Disabilities Education Act, 2008. VA: U.S. Department of Education.

van Loon, J. (2008). The Arduin benchmark. In R. L. Schalock, J. R. Thompson, \& M. J. Tassé (Eds.), Resource allocation and the Supports Intensity Scale: Four papers on issues and approaches (pp. 19-21). Washington, DC: American Association on Intellectual and Developmental Disabilities.

van Loon, J., Claes, C., Vandevelde, S., Van Hove, G., \& Schalock, R. (2010). Assessing individual support needs to enhance personal outcomes. Exceptionality, 18, 193-202. doi:10.1080/09362835.2010.513924

Verdugo, M., Arias, B., Ibanez, A., \& Gomez, S. (2006). Validation of the Spanish version of the Supports Intensity Scale. Journal of Applied Research in Intellectual Disabilities, 19(3), 274 
Verdugo, M., Arias, B., Ibanez, A., \& Schalock, R. L. (2010). Adaptation and psychometric properties of the Spanish version of the Supports Intensity Scale (SIS). American Association on Intellectual and Developmental Disabilities, 115, 496-503. doi:10.135/1944-7558-115.6.496

VOR. (2013). Emerging threat: Use of the Supports Intensity Scale assessment tool for ICF/MD discharge. Retrieved from http://www.vor.net/get-help/community-resources/284emerging-threat-use-of-the-supports-intensity-scale-assessment-tool-for-icfmr-discharge

Ward, M. J. (2005). An historical perspective of self-determination in special education: Accomplishments and challenges. Research \& Practice for Persons with Severe Disabilities, 30(3), 108-112.

Ward, R. L., Nichols, A. D., \& Freedman, R. I. (2010). Uncovering health care inequalities among adults with intellectual and developmental disabilities. Health \& Social Work, $35(4), 280-290$.

Waterhouse, L. (2006). Inadequate evidence for Multiple Intelligences, Mozart Effect, and Emotional Intelligence theories. Educational Psychologist, 41(4), 247-255.

Weber, L., \& Stern, J. (2008). Washington's residential resource allocation model. In R. L. Schalock, J. R. Thompson, \& M. J. Tassé (Eds.), Resource allocation and the Supports Intensity Scale: Four papers on issues and approaches (pp. 11-18). Washington, DC: American Association on Intellectual and Developmental Disabilities.

Wehman, P., Kregel, J., Banks, P. D., Hill, M., \& Moon, M. S. (1987). Sheltered versus supported work programs: A second look. Rehabilitation Counseling Bulletin, 31, 42-53.

Wehmeyer, M. L. (2013). The story of intellectual disability: An evolution of meaning, understanding, \& public perception. Baltimore, MD: Paul H. Brookes.

Wehmeyer, M. L., Abery, B. H., Zhang, D., Ward, K., Willis, D., Hossain, A., ... Calkins, C. (2011). Promoting self-determination and moderating variables that impact efforts to promote self-determination. Exceptionality, 19, 19-30. doi: $10.1080 / 09362835.20122 .537225$

Wehmeyer, M., Chapman, T. E., Little, T. D., Thompson, J. R., Schalock, R., \& Tassé, M. J. (2009). Efficacy of the Supports Intensity Scale (SIS) to predict extraordinary support needs. American Journal on Intellectual and Developmental Disabilities, 114(1), 3-14.

Wehmeyer, M. L., \& Field, S. (2007). Self-determination: Instructional and assessment strategies. Thousand Oaks, CA: Corwin.

Wehmeyer, M. L., \& Schalock, R. L. (2013). The parent movement: Late modern times (1950 CE to 1980 CE). In M. L. Wehmeyer (Ed.). The story of intellectual disability (pp. 187-232). Baltimore, MD: Paul H. Brookes. 
Wehmeyer, M. L., Tassé, M. J., Davies, D. K., \& Stock, S. (2012). Support needs of adults with intellectual disability across domains: The role of technology. Journal of Special Education Technology, 27(2), 11-21.

Wei, Y., Oakland, T., \& Algina, J. (2008). Multigroup confirmatory factor analysis for the Adaptive Behavior Assessment System - II Parent Form, ages 5-12. American Journal on Mental Retardation, 113(3), 178-186.

Weiss, J. A., Lunsky, Y., Tassé, M. J., \& Durbin, J. (2009). Support for the construct validity of the Supports Intensity Scale based on clinician rankings of need. Research in Developmental Disabilities, 30, 933-941. doi:10.1016/j.ridd.2009.01.007

WHO. (2012). International Classification of Functioning, Disability, and Health (ICF). Retrieved from http://www.who.int/classifications/icf/en/

Williams, V. J. (2009). Fatalism: Anthropology, psychology, sociology and the IQ controversy. Journal of African American Studies, 13, 90-96. doi: 10.1007/s12111-008-9074-1

Wolfensberger, W. (1983). Social role valorization: A proposed new term for the principle of normalization. Mental Retardation, 21, 234-239. doi: 10.1352/1934-9556-49.6.435

Yell, M. L. (2006). The law and special education (2nd ed.). Upper Saddle River, NJ: Pearson.

Ysseldyke, J. E., Algozzine, B., \& Thurlow, M. L. (2000). Critical issues in special education (3rd ed.). Boston, MA: Houghton Mifflin.

Zheng, X., Chen, R., Li, N., Du, W., Pei, L., Zhang, J., ... Yang, R. (2012). Socioeconomic status and children with intellectual disability in China. Journal of Intellectual Disability Research, 56, 212-220. doi: 10.1111/j.1365-2788.2011.01470.x

Zigler, E., Balla, D., \& Hodapp, R. (1984). On the definition and classification of mental retardation. American Journal of Mental Deficiency, 89, 215-230. 
APPENDIX A

GRAPHIC REPRESENTATIONS OF THE LATENT FACTOR MODELS 
Model 1 - 7-Factor Model Aligned With the Subscale Structure of the SIS

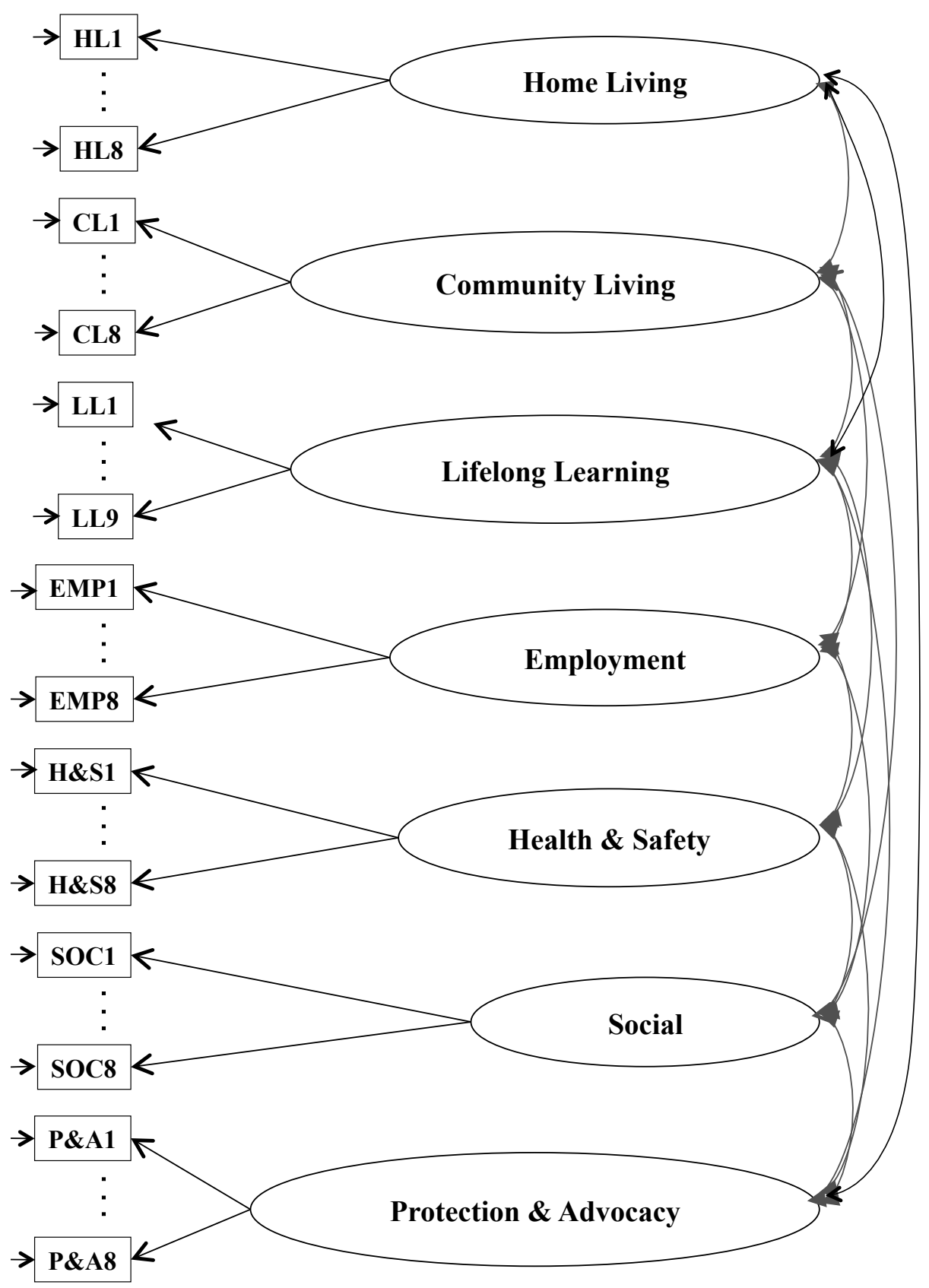


Model 2 - 7-Factor Hierarchical Model

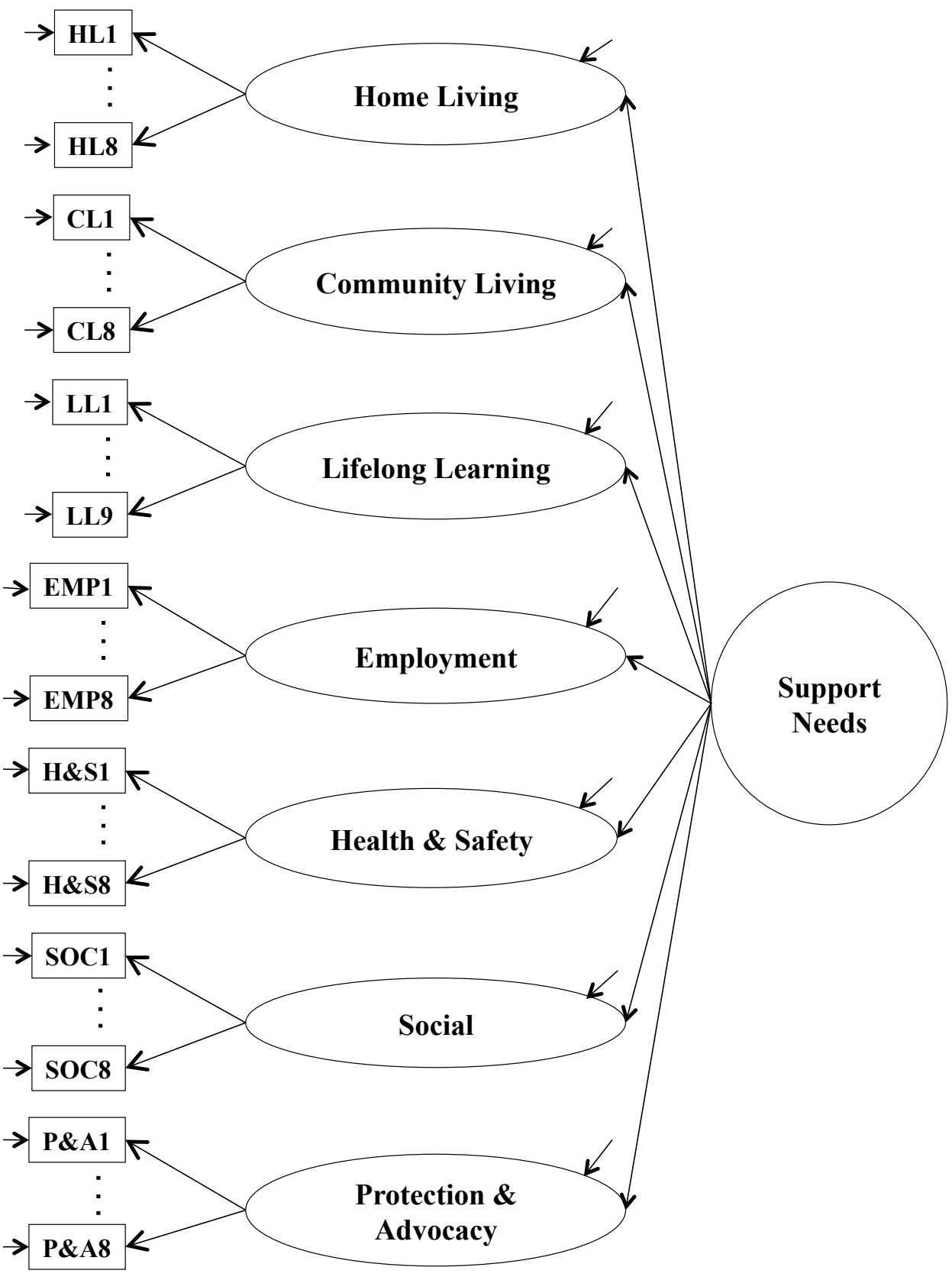

Note. This is an effect-indicator model; the arrows go from the latent factors to the observed variables 
Model 3 - 5-Factor Model Based on the 4-Factor Structure With a Subset of SIS and the P\&A Scale

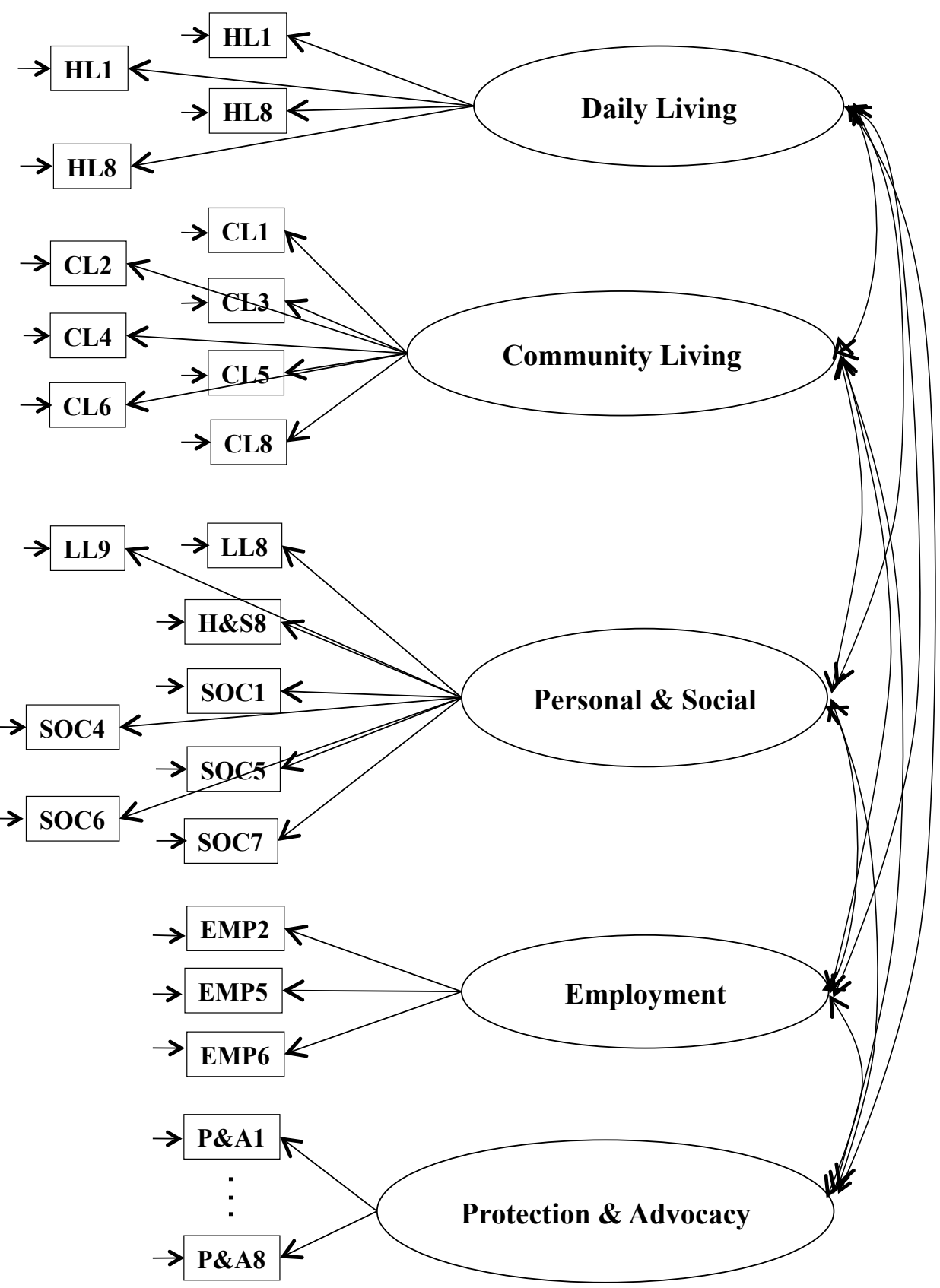


Model 4 - 5-Factor Model Based on the 4-Factor Structure With All SIS Items and the P\&A Scale

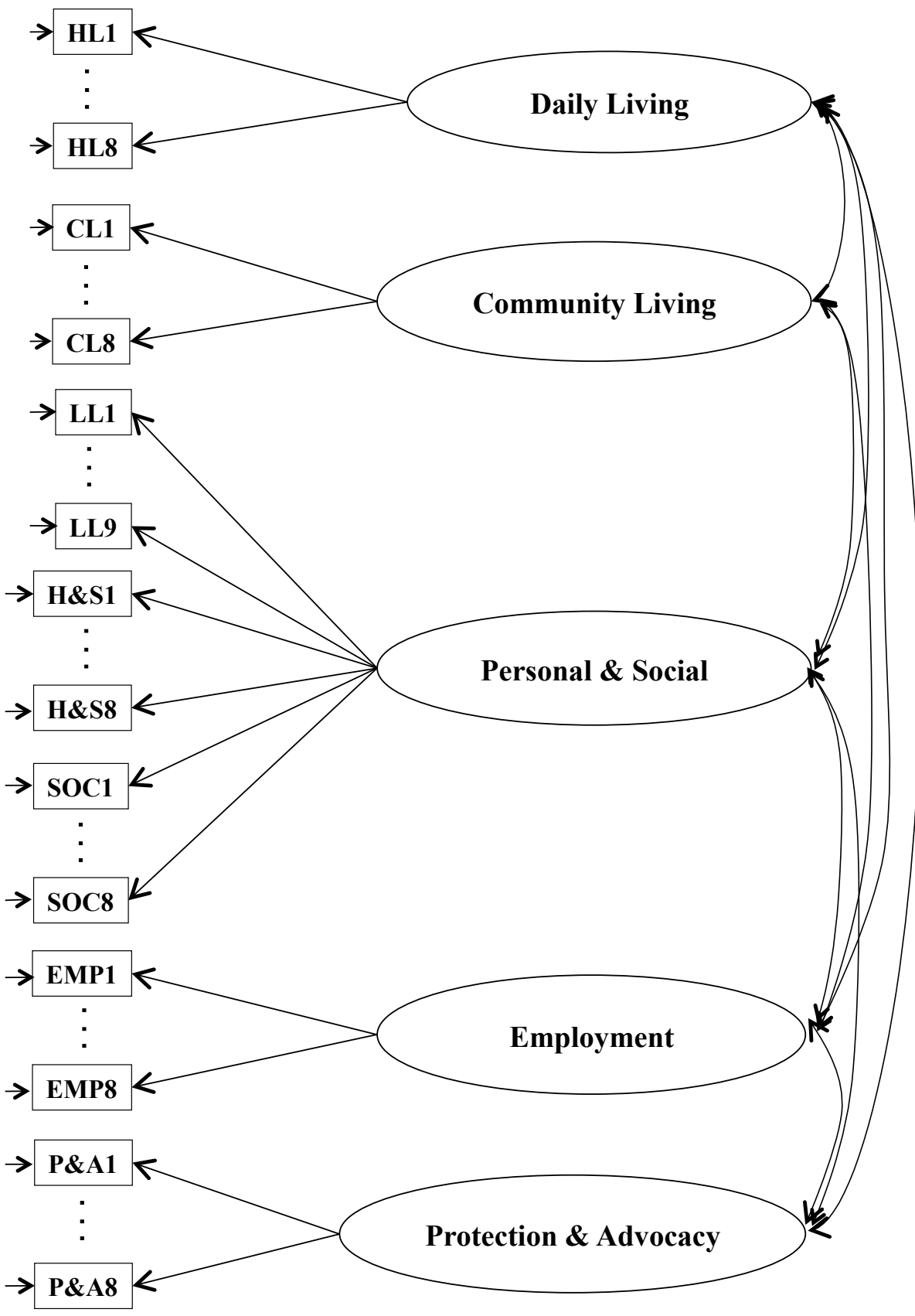


Model 5 - Single-Factor Model

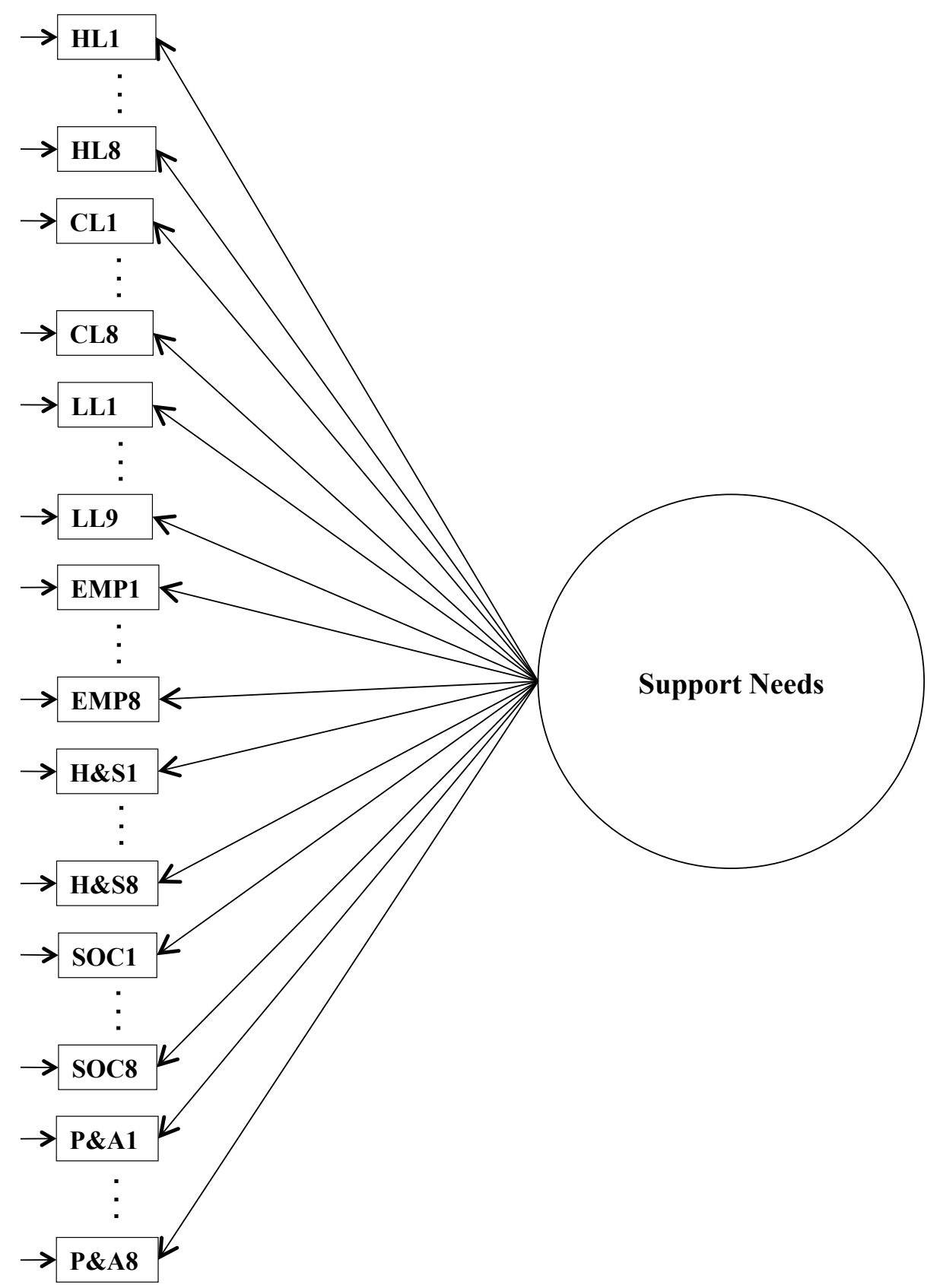


APPENDIX B

SAMPLE SUMMARY OF FINDING FROM SIS ASSESSMENT 


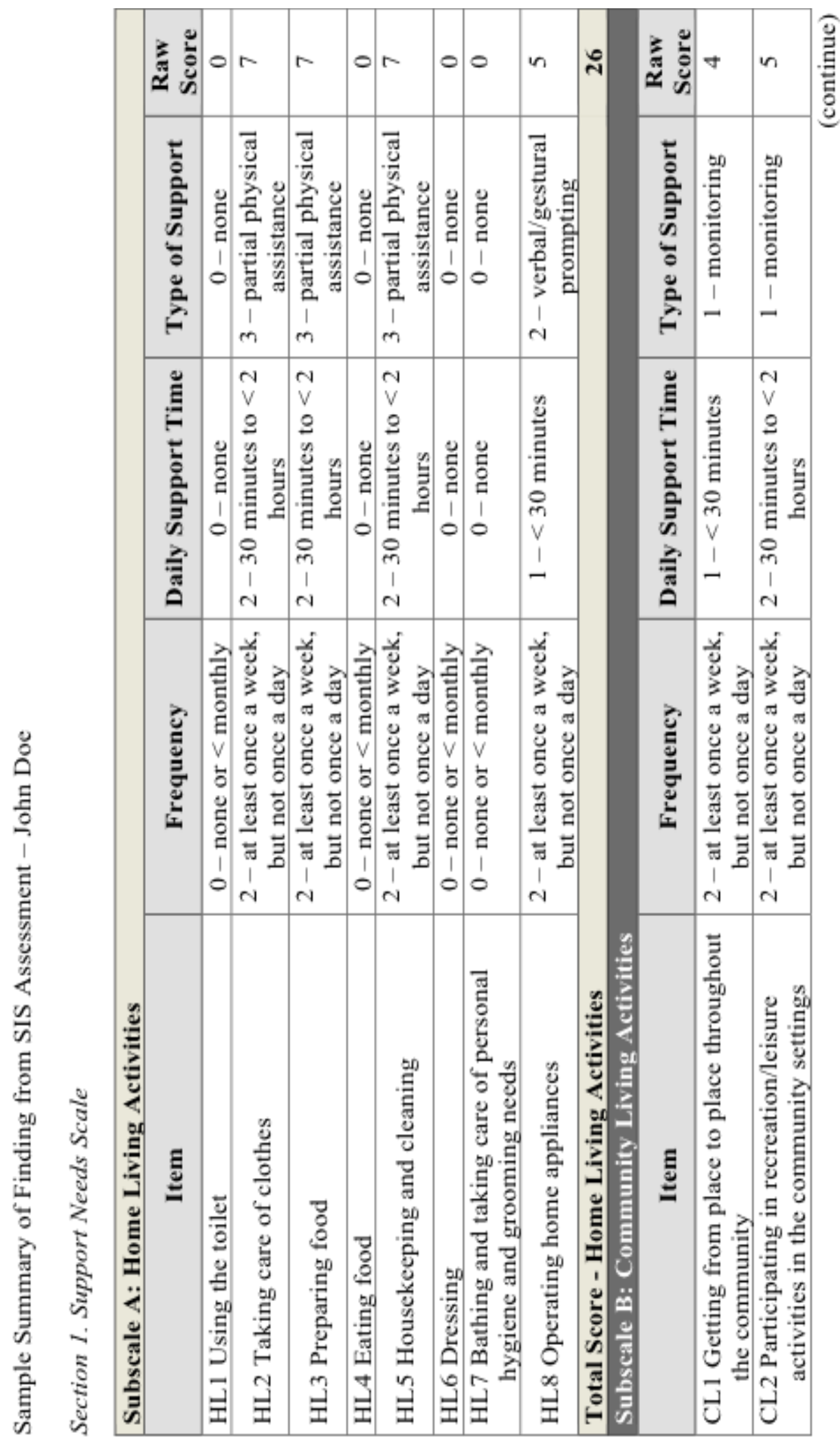




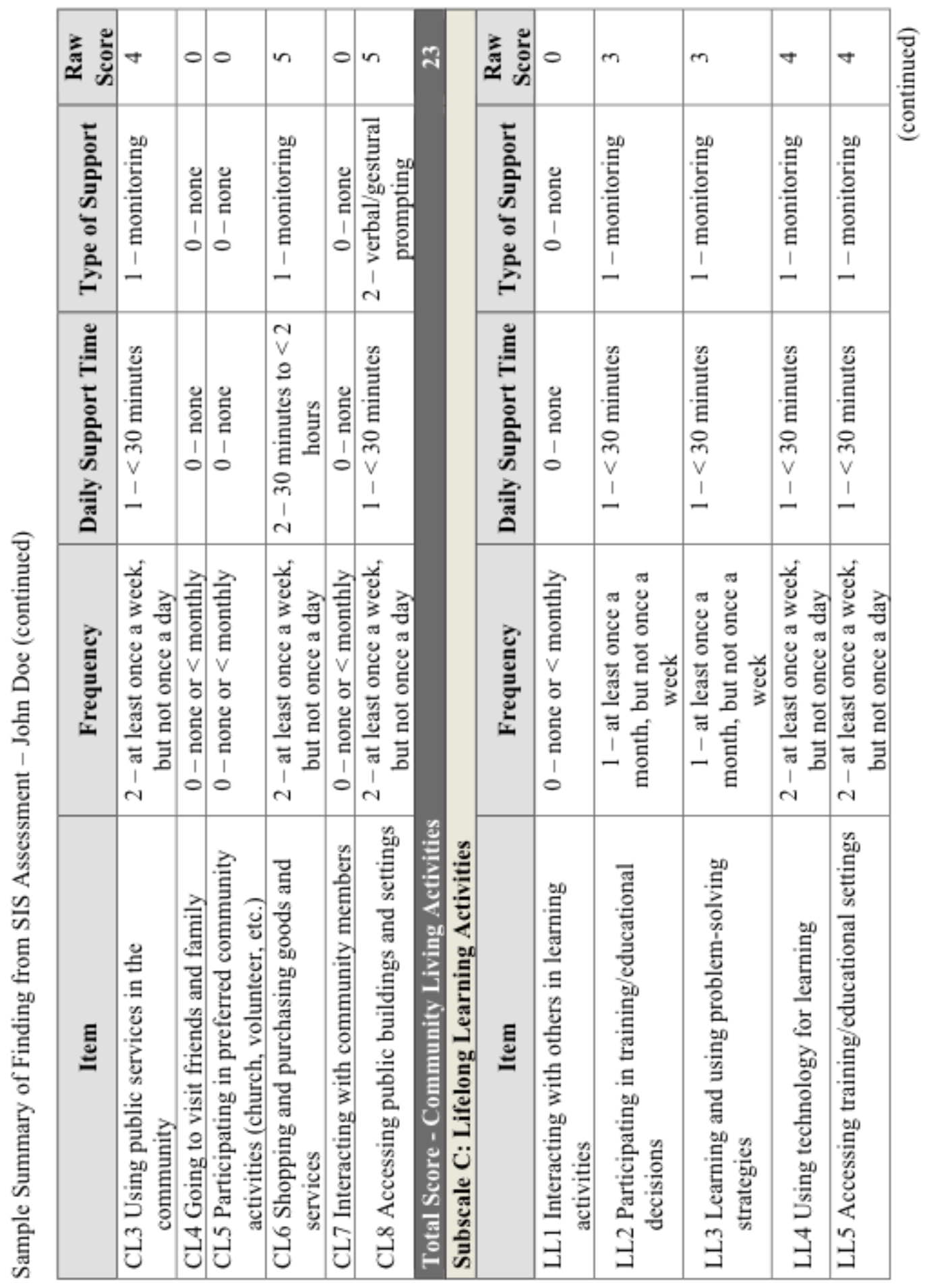




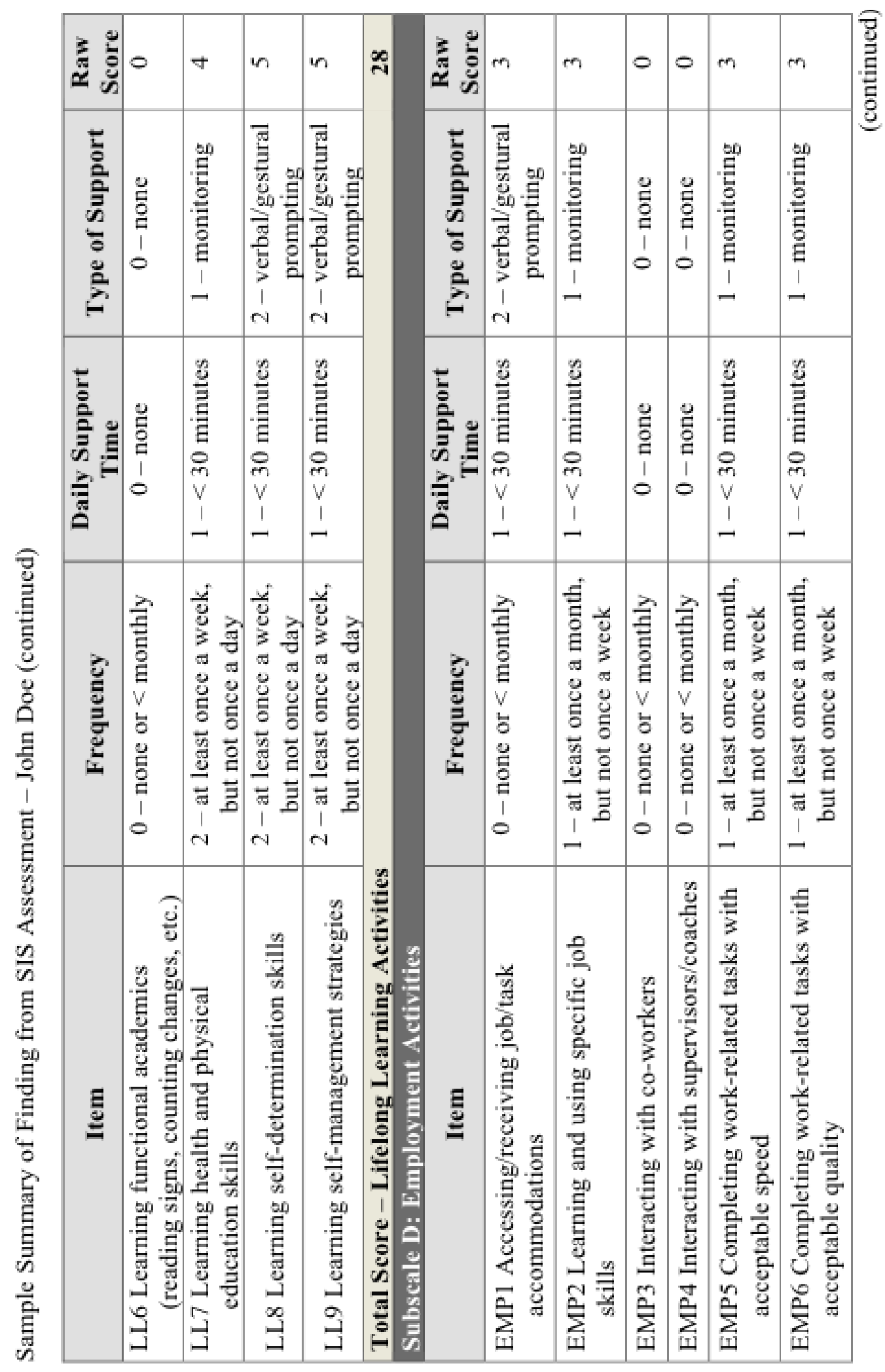




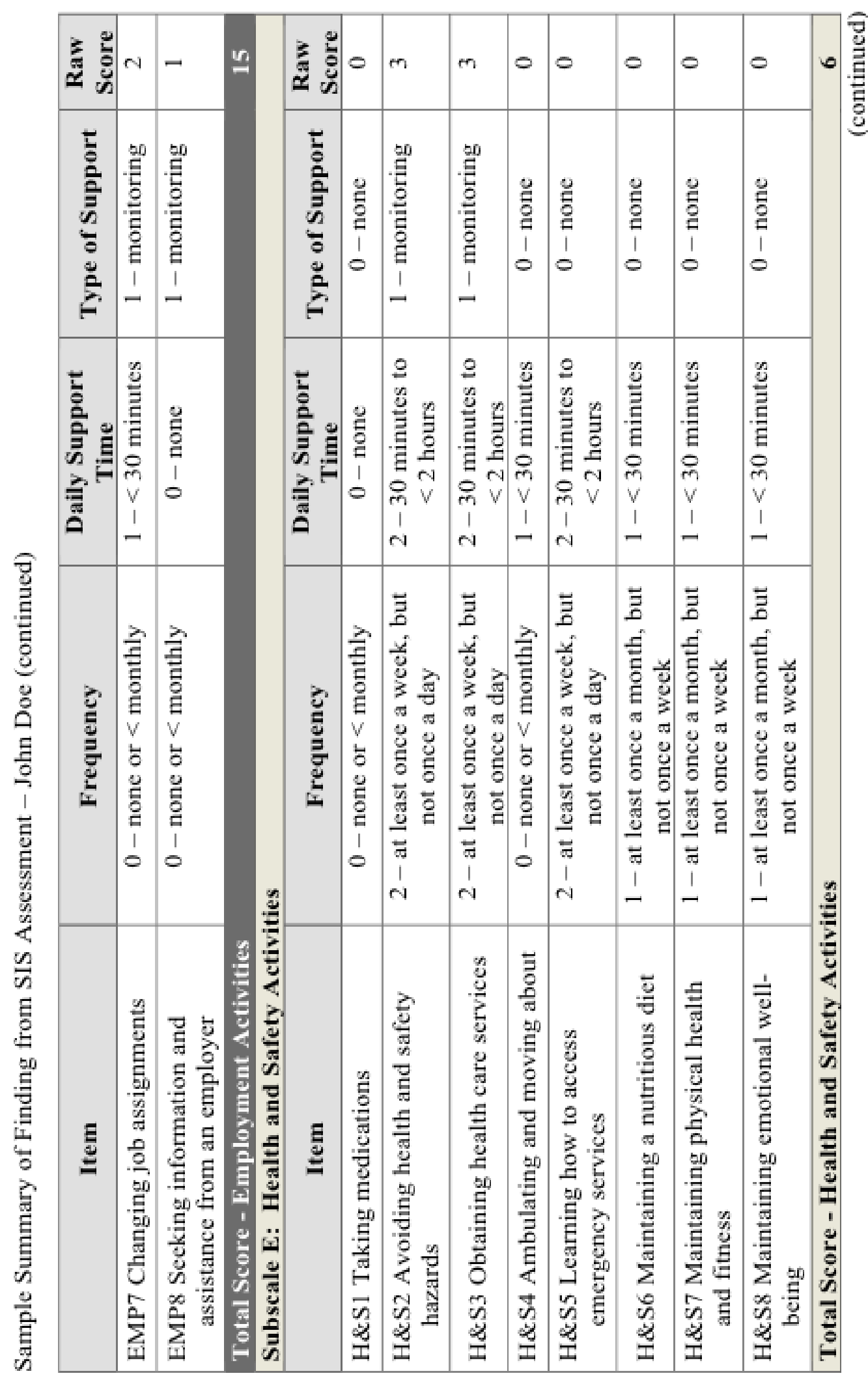




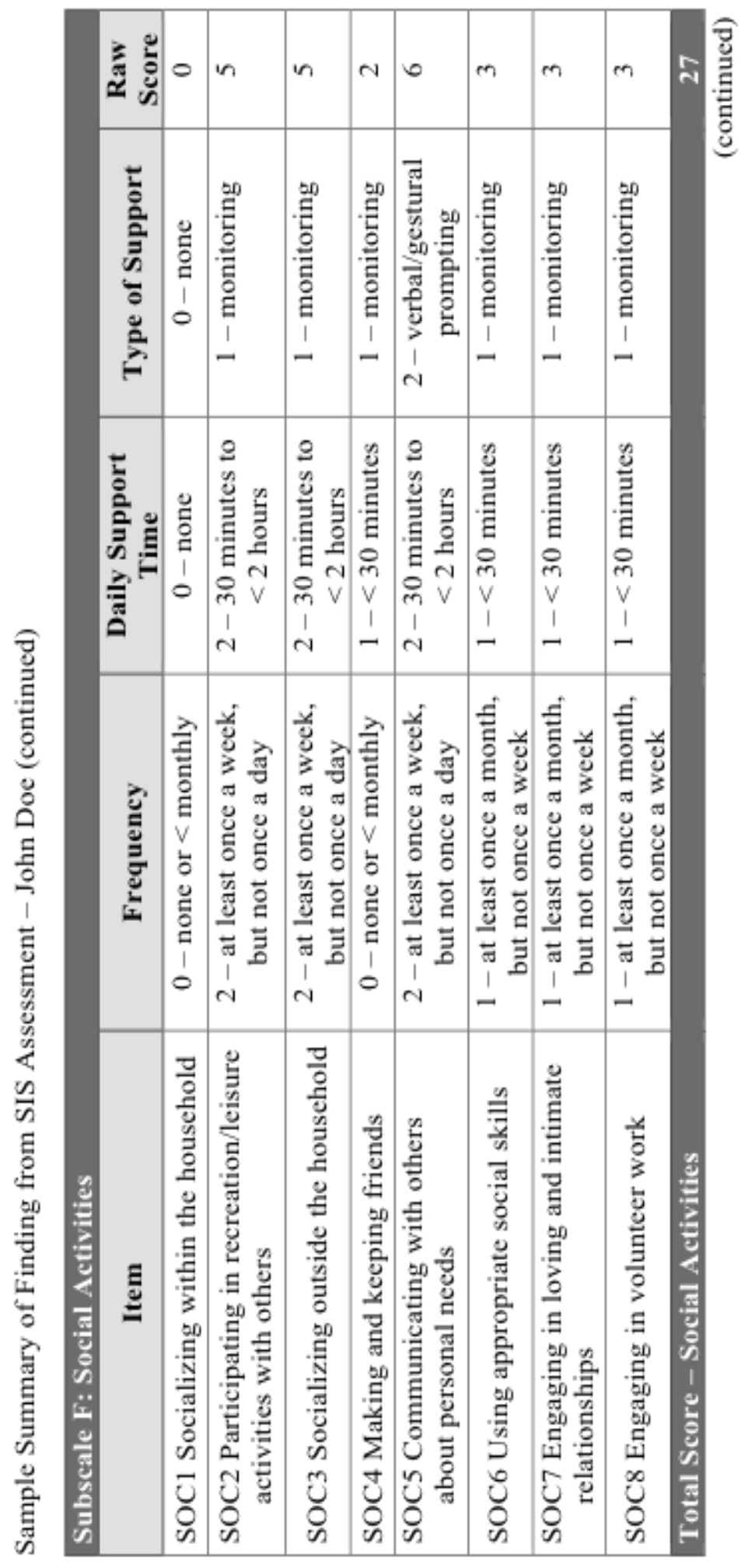




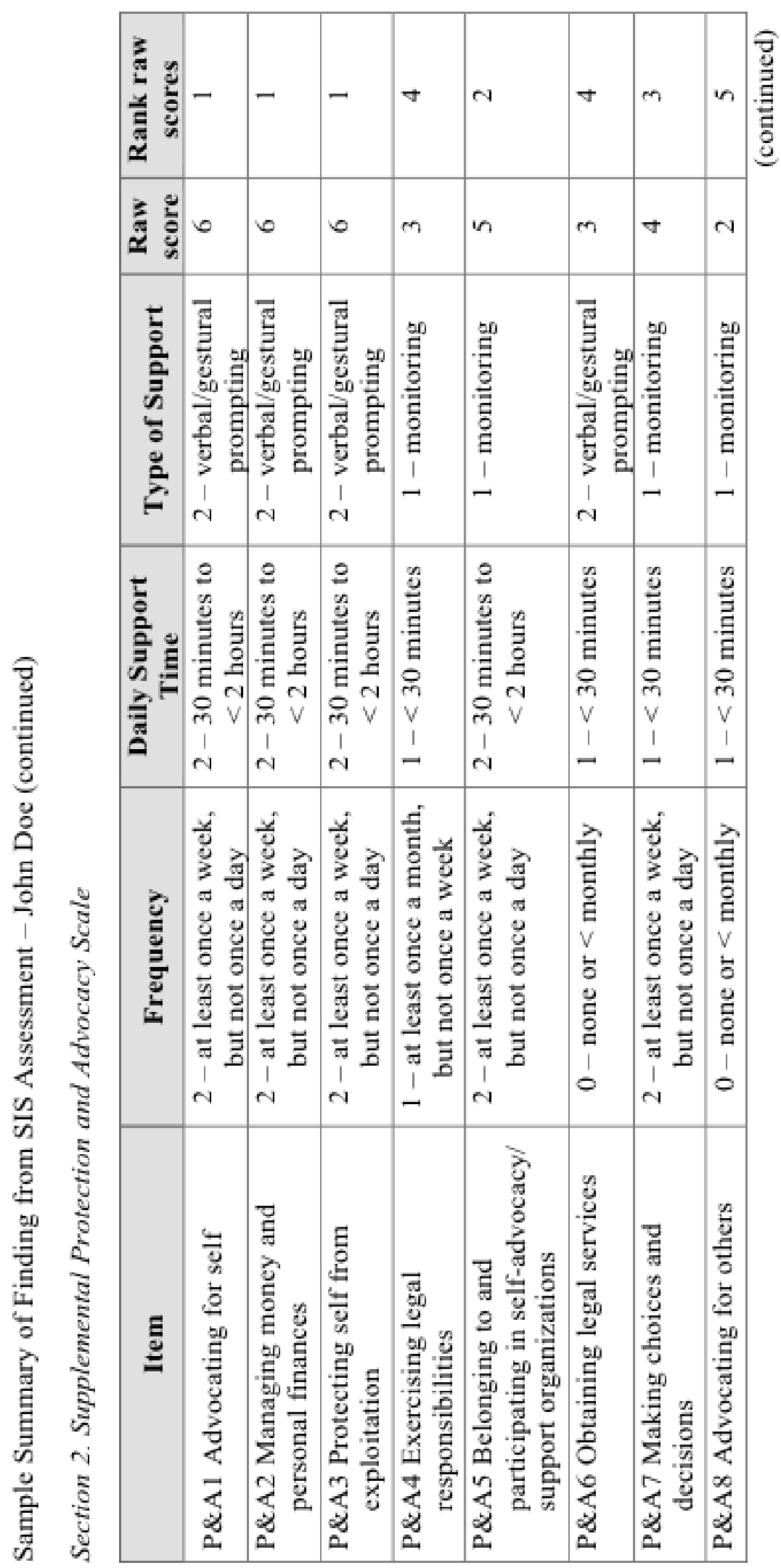




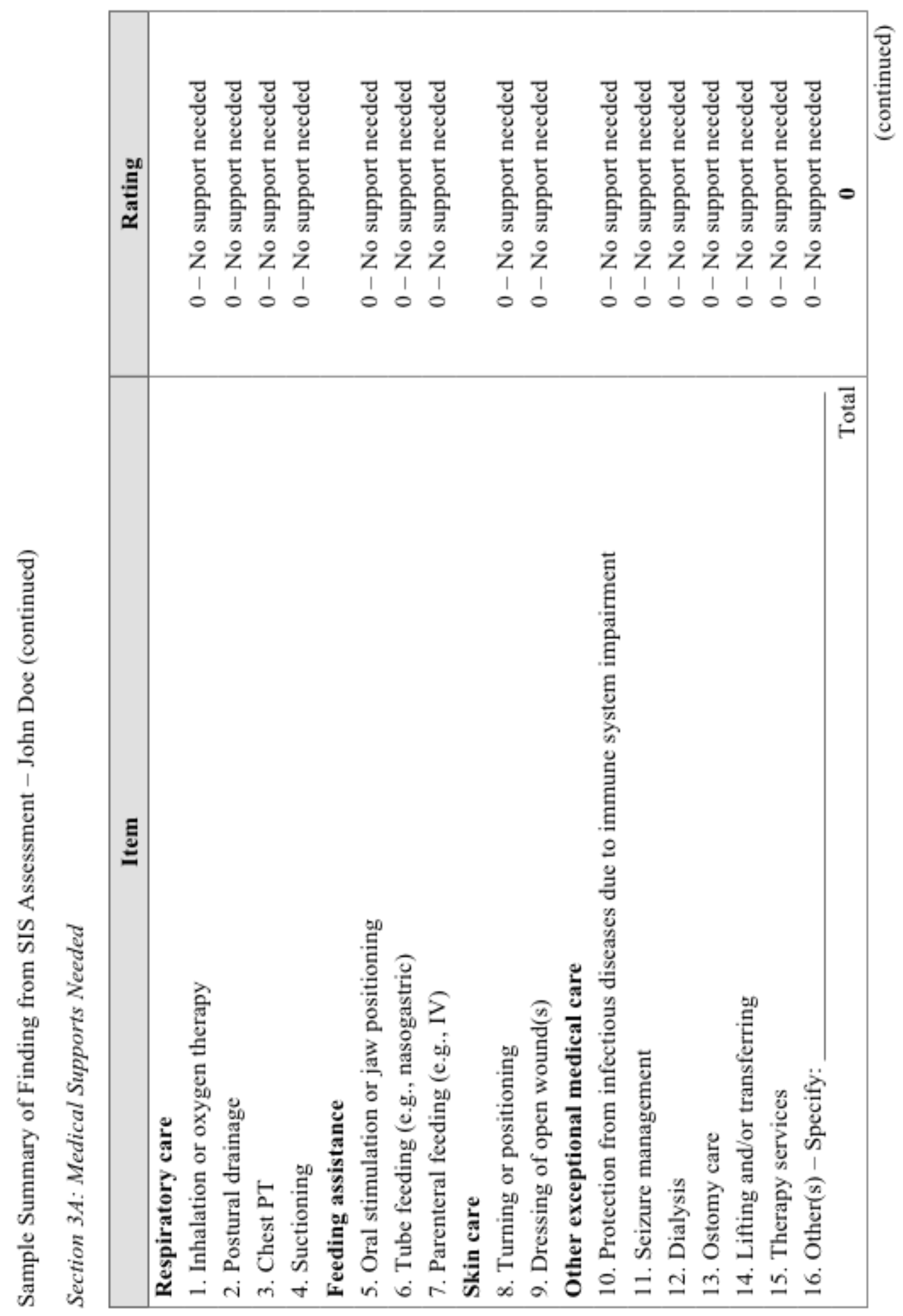




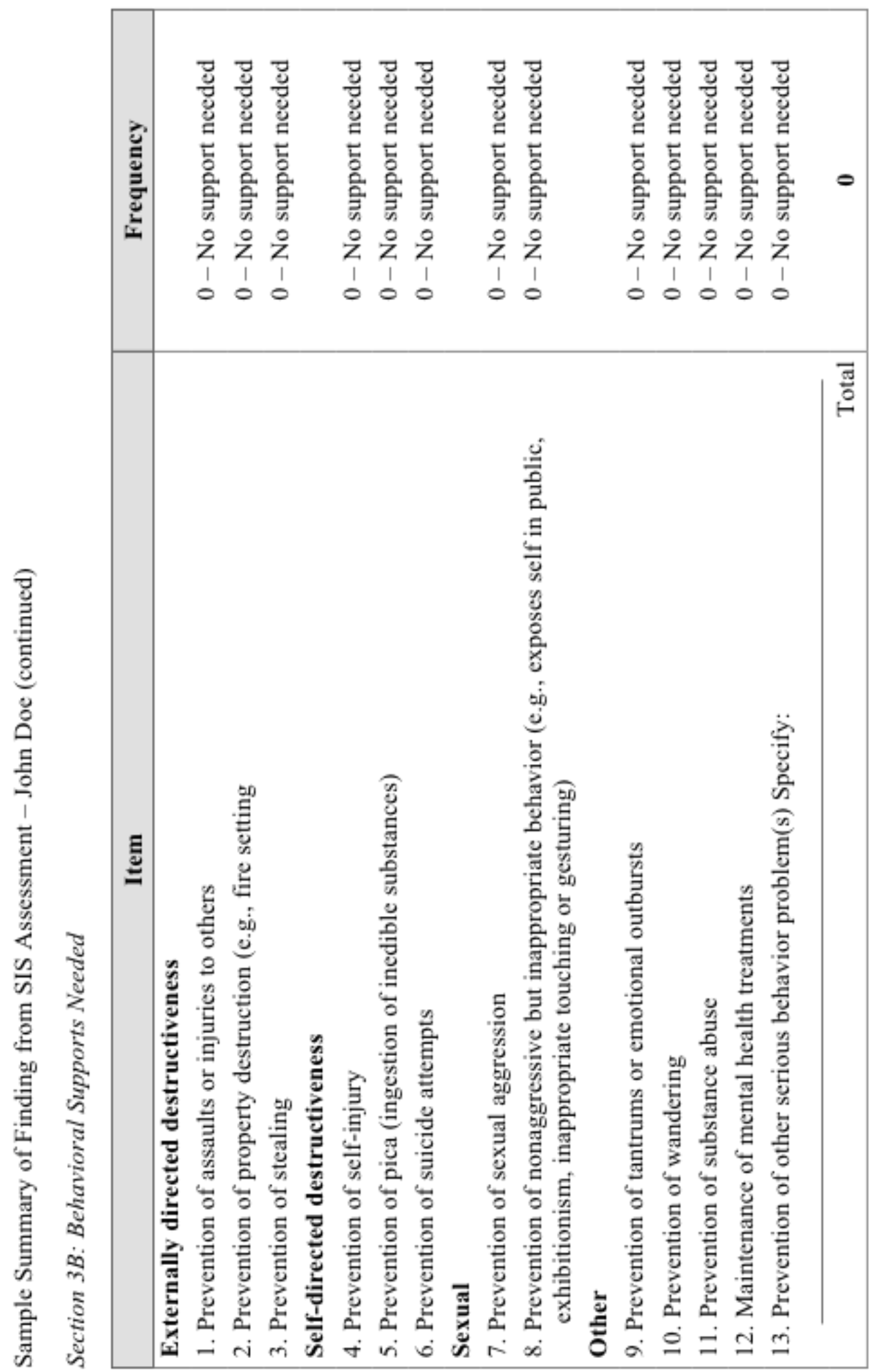

